\title{
CRISPR-Cas9-mediated protein tagging in human cells for RESOLFT nanoscopy and the analysis of mitochondrial prohibitins
}

\author{
Dissertation \\ for the award of the degree \\ "Doctor rerum naturalium" \\ Division of Mathematics and Natural Sciences \\ of the Georg-August-Universität Göttingen \\ within the doctoral program Molecular Biology \\ of the Georg-August University School of Science (GAUSS)
}

submitted by

Michael Ratz

from Sangerhausen

Göttingen, 2015 
Thesis Committee:

Prof. Stefan Jakobs

Department of NanoBiophotonics

Mitochondrial Structure and Dynamics Group

Max Planck Institute for Biophysical Chemistry, Göttingen

Prof. Peter Rehling

Department of Cellular Biochemistry

University Medical Center Göttingen

Prof. Stefan W. Hell

Department of NanoBiophotonics

Max Planck Institute for Biophysical Chemistry, Göttingen

Members of the examination board:

Prof. Stefan Jakobs (Referee)

Prof. Peter Rehling (Co-referee)

Prof. Stefan W. Hell

Prof. Michael Meinecke

Department of Cellular Biochemistry

University Medical Center Göttingen

Prof. Detlef Doenecke

Department of Molecular Biology

University Medical Center Göttingen

Prof. Markus T. Bohnsack

Department of Molecular Biology

University Medical Center Göttingen

Date of the oral examination: $17^{\text {th }}$ December 2015 
You can observe a lot by watching. Yogi Berra 



\section{Table of contents}

Summary _ iv

1. Introduction 1

1.1. Genome engineering using CRISPR-Cas9................................................... 1

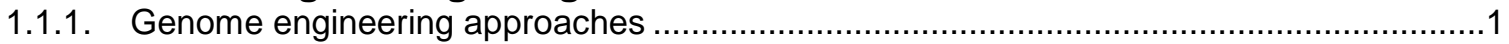

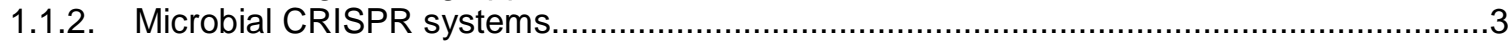

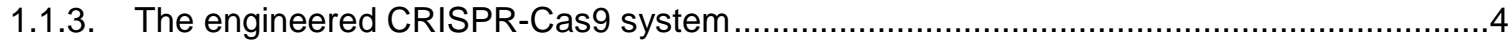

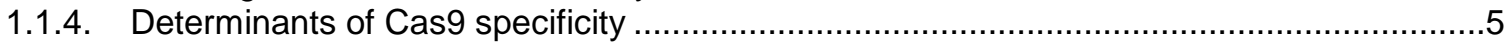

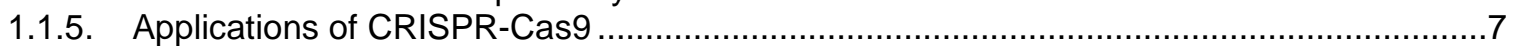

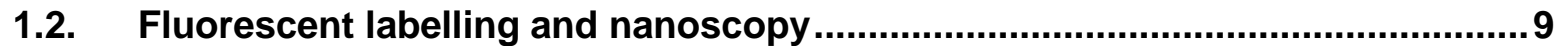

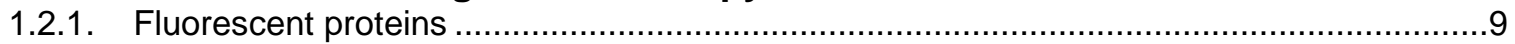

1.2.2. Reversibly switchable fluorescent proteins........................................................... 11

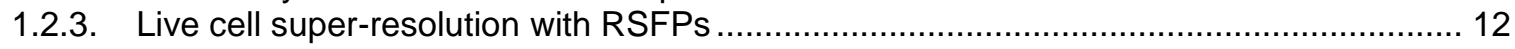

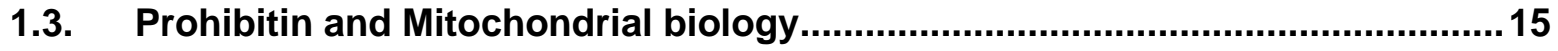

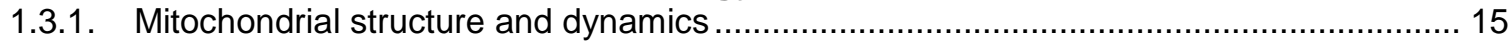

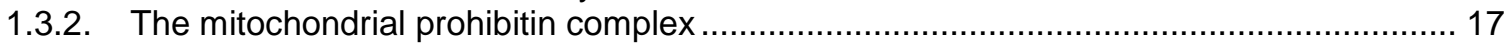

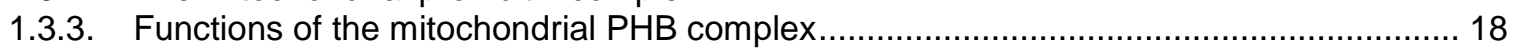

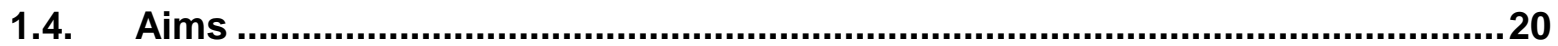

2. Materials and Methods _ 22

2.1. Bacterial and human cell culture..............................................................22

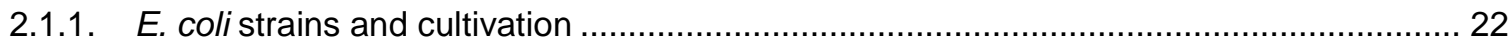

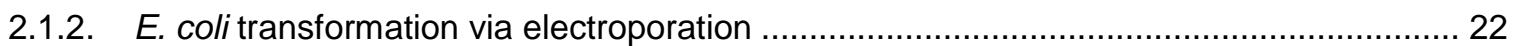

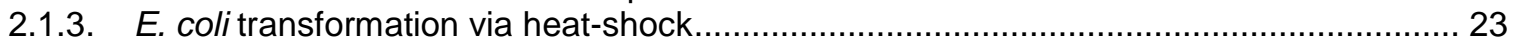

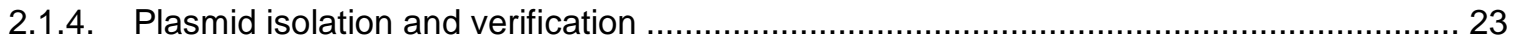

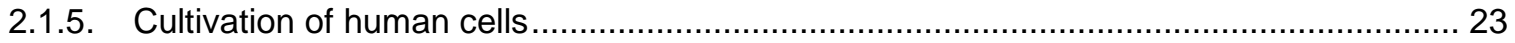

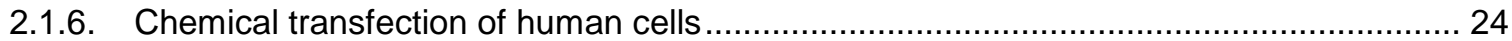

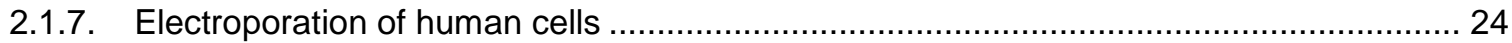

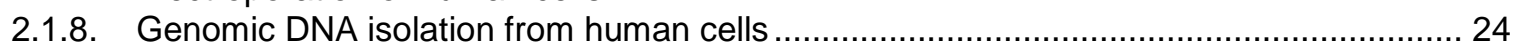

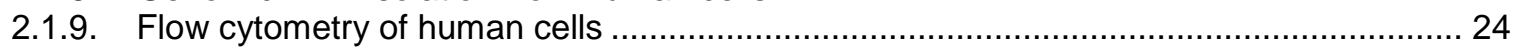

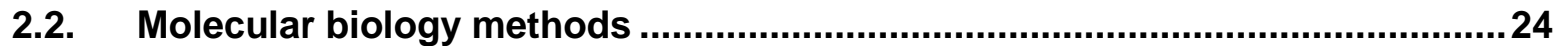

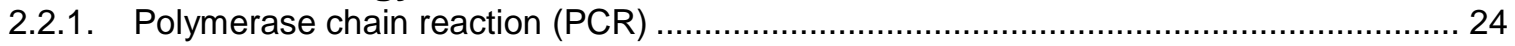

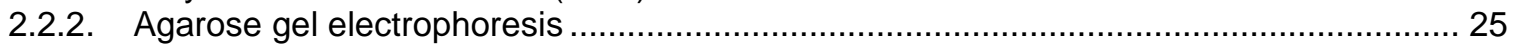

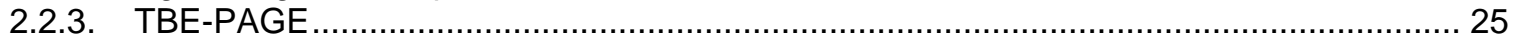

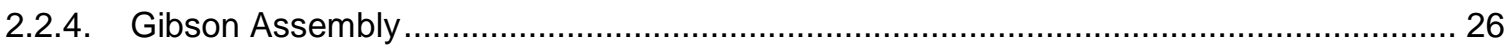

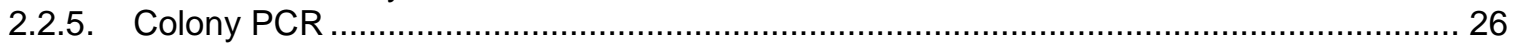

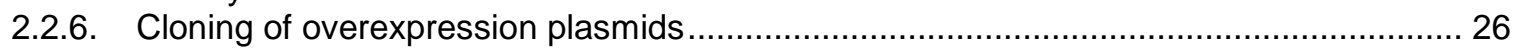

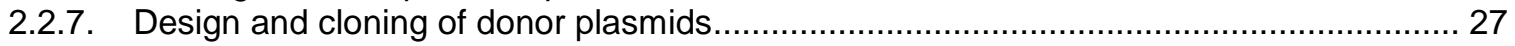

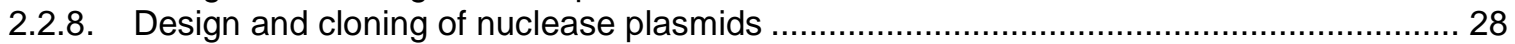

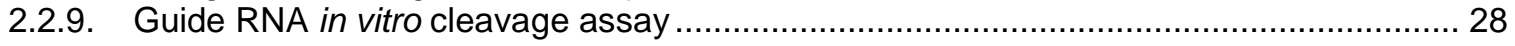

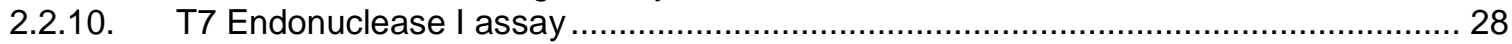

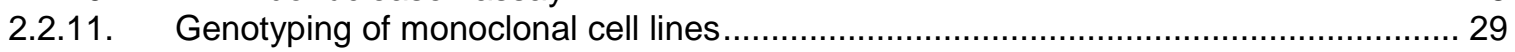

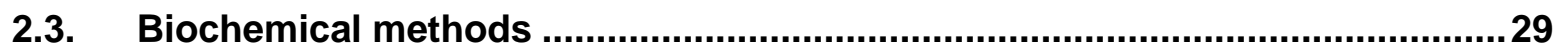

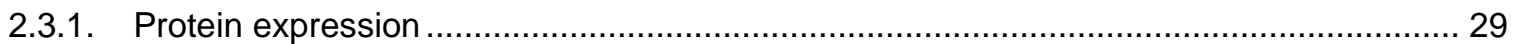

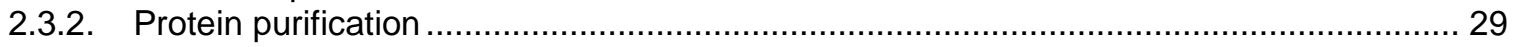

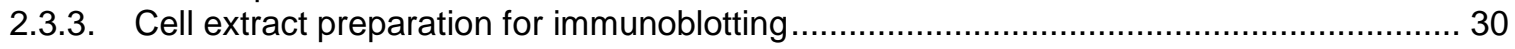

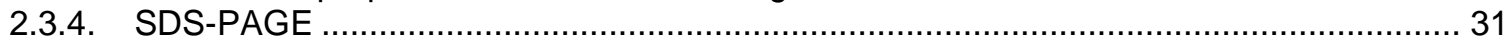

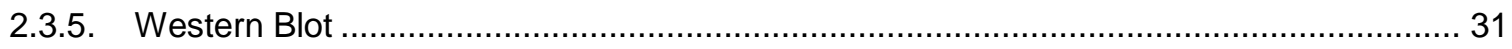




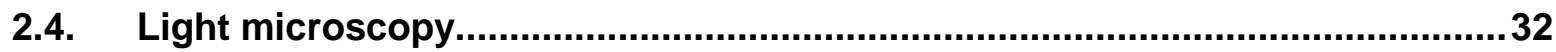

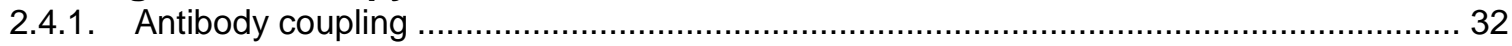

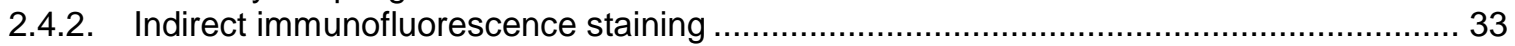

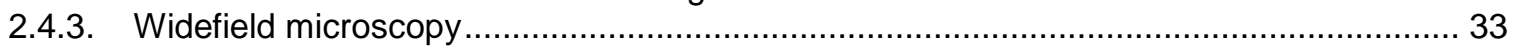

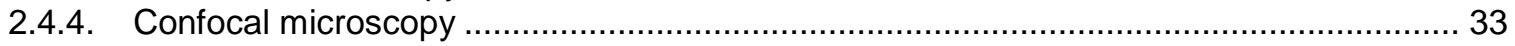

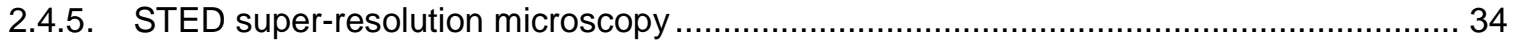

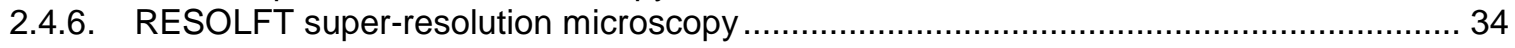

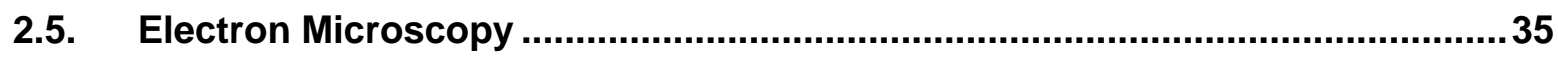

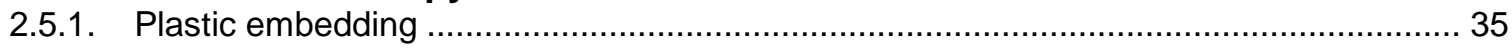

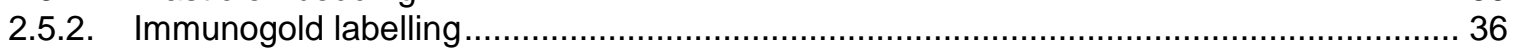

3. Results 37

3.1. Genomic tagging using zinc finger nucleases (ZFNs) ..................................37

3.2. Establishment of a general workflow for genome editing of human cells using

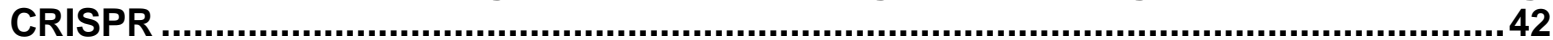

3.2.1. Target selection and generation of gRNAs ............................................................ 42

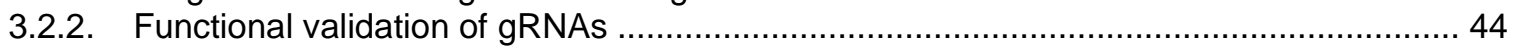

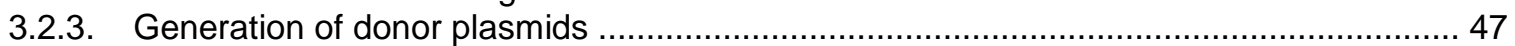

3.2.4. Clone isolation and clonal expansion …………….................................................... 48

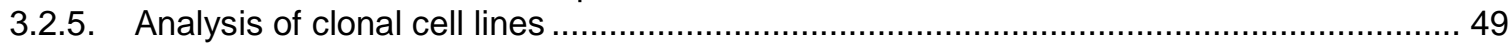

\subsection{CRISPR-Cas9-mediated endogenous protein tagging for super-resolution} imaging of living human cells

3.3.1. Endogenous tagging of HMGA1, VIM and ZYX with rsEGFP2 …............................5 51

3.3.2. DNA sequencing of endogenously tagged cell lines ................................................ 58

3.3.3. Flow cytometry of endogenously tagged and overexpressing cells ..............................65

3.3.4. Confocal microscopy of endogenously tagged and overexpressing cells .......................68

3.3.5. RESOLFT imaging of HMGA1-, VIM- and ZYX-rSEGFP2 cells ................................. 70

3.4. Localization, dynamics and abundance of mitochondrial prohibitins.............73

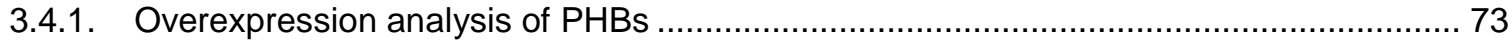

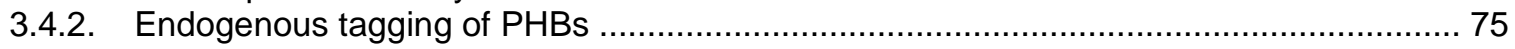

3.4.3. Sub-mitochondrial localization of prohibitins in human cells ........................................ 85

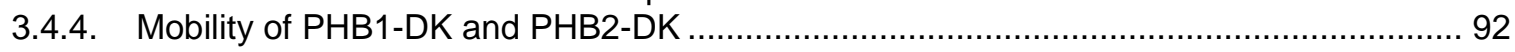

3.4.5. Nuclear localization of PHB2 in HeLa cells is an overexpression artefact ........................99

3.4.6. Prohibitin quantification in single cells and cristae membranes ................................... 96

4. Discussion 105

4.1. RESOLFT imaging of endogenously tagged $\beta$-actin...................................... 105

4.2. CRISPR-based endogenous protein tagging..............................................107

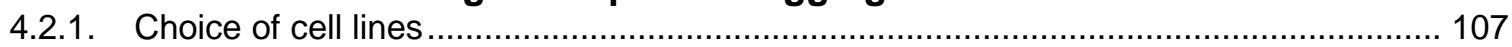

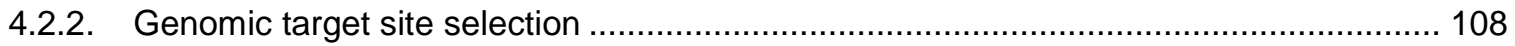

4.2.3. In vivo vs in vitro functional gRNA validation......................................................... 109

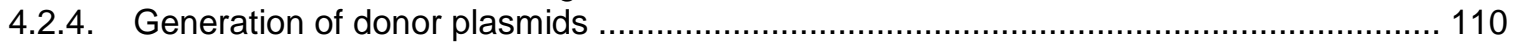

4.2.5. Clone isolation via FACS and characterization of monoclonal cell lines ....................... 111

4.3. Endogenous tagging for live cell super-resolution microscopy...................112

4.3.1. Increasing integration frequencies and survival rates after single cell sorting ............... 112

4.3.2. Gene editing of HMGA1, VIM and ZYX using CRISPR is highly specific .................... 112

4.3.3. Endogenous tagging prevents overexpression artefacts ......................................... 113

4.3.4. RESOLFT allows nanoscale imaging at endogenous expression levels ..................... 114

4.4. Quantitative analysis of mitochondrial prohibitins in human cells ...............115

4.4.1. Overexpression of PHB1-DK or PHB2-DK induces aberrant mitochondria .................. 116 
4.4.2. Endogenously tagged PHB1-DK and PHB2-DK clones are heterozygous and exhibit

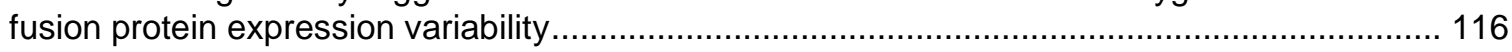

4.4.3. Endogenously tagged PHBs are found in mitochondria .......................................... 117

4.4.4. Prohibitins form immobile clusters in the inner mitochondrial membrane .................... 118

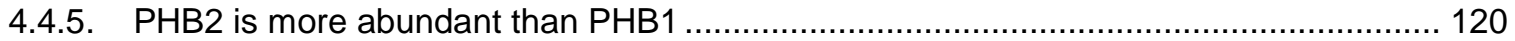

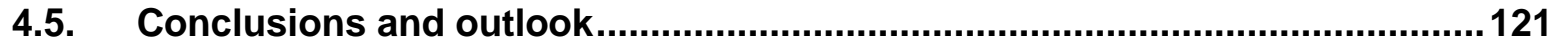

5. References 123

6. Supplementary information

List of figures 158

List of tables $\quad 160$

$\begin{array}{ll}\text { Abbreviations } & 161\end{array}$

Acknowledgements $\quad 164$

Affidavit 165

Curriculum vitae 166 


\section{Summary}

Ectopic overexpression of fluorescent fusion proteins for live cell imaging studies often leads to a multitude of artefacts, but protein expression at endogenous levels in mammalian cells was difficult to achieve so far. To avoid common problems associated with overexpression, this study used the CRISPR-Cas9 genome engineering system for site-specific endogenous protein tagging in human cells. First, a general workflow for genome editing was established and then applied to generate heterozygous and homozygous human knock-in cells that express a fluorescent fusion from a genomic locus. Three human genes (HMGA1, VIM and ZYX) were tagged with the reversibly switchable fluorescent protein rsEGFP2 and the benefit of endogenous over ectopic expression demonstrated using flow cytometry and confocal microscopy. Moreover, low light intensity RESOLFT super-resolution microscopy could be applied to study nanoscale protein dynamics at physiologically relevant protein expression levels in living knock-in cells.

CRISPR-mediated endogenous tagging was crucial to investigate the localization, dynamics and abundance of mitochondrial prohibitin 1 and 2, PHB1 and PHB2, in human cells. While overexpression of PHB1 and PHB2 caused aberrant mitochondria, endogenous tagging of prohibitins with the fluorescent protein Dreiklang (DK) restored wildtype mitochondrial morphology. Overexpression of PHB2-DK and human estrogen receptor a caused a mislocalization of PHB2-DK in the nucleus of HeLa cells, but also this artefact was not observed in endogenously tagged PHB2-DK HeLa cells. Homologous recombination frequencies for PHB1 and PHB2 tagging were remarkably high and a number of heterozygous PHB1-DK and PHB2-DK knock-in clones could be generated. STED superresolution microscopy uncovered the hitherto unknown organization of individual prohibitins into clusters. Dual-color STED imaging demonstrated a colocalization of tagged and untagged PHB1 and PHB2 indicating that PHB complex formation is not affected by protein tagging. Intriguingly, the vast majority of prohibitins is found at the mitochondrial cristae membrane where they form exceptionally static protein assemblies. Surprisingly, the global amount of PHB2 was found to be 4-5 times higher than that of PHB1, which is in contrast to in vitro studies conducted on purified yeast prohibitins. After integrating the results on prohibitin localization and abundance with morphological data about the ultrastructural organization of mitochondria and under the assumption that human prohibitins form a $1 \mathrm{MDa}$ complex, it was estimated that about 31-36 individual PHB complexes occupy a single cristae membrane. 


\section{Introduction}

\subsection{Genome engineering using CRISPR-Cas9}

\subsubsection{Genome engineering approaches}

Genome engineering or gene editing refers to the process of making site-specific changes to the genomic information of cells and organisms. Pioneering experiments demonstrated that the genome can be altered precisely by homologous recombination (HR) between two similar DNA molecules (Smithies et al., 1985; Thomas and Capecchi, 1986; Mansour et al., 1988). HR introduces highly precise modifications, but occurs at extremely low frequencies in mammalian cells $\left(10^{-6}\right.$ to $\left.10^{-9}\right)$ and often requires the use of drug-selection markers (Capecchi, 1989). Later studies showed that HR-mediated genome modifications could be greatly enhanced by the introduction of site-specific DSBS mediated by naturally occurring endonucleases (Plessis et al., 1992; Rouet et al., 1994; Choulika et al., 1995).

The first site-specific nucleases were chimeric fusion proteins between the non-specific nuclease domain of the restriction enzyme Fokl and a zinc finger DNA recognition domain (Kim et al., 1996). Zinc finger nucleases (ZFNs) could be designed to recognize a specific DNA sequence of interest and were found to efficiently enhance locus-specific HR (Figure 1) (Bibikova et al., 2001; Bibikova et al., 2003). Furthermore, the site-specific introduction of a DSB was shown to induce insertion/deletion mutations (indels) in absence of a homologous repair template via the error-prone nonhomologous end-joining pathway (NHEJ) (Bibikova et al., 2002). Zinc finger proteins recognize specific DNA sequences through protein-DNA interactions and consist of individual repeats targeting 3 nucleotides of DNA (Christy and Nathans, 1989; Pavletich and Pabo, 1991). Assembly of individual repeats into larger arrays leads to altered binding specificities due to crosstalk between adjacent repeats (Maeder et al., 2008). Development and validation of zinc finger proteins with new sequence specificities is therefore inherently difficult and remains a major challenge that requires expert knowledge and extensive screening (Gonzalez et al., 2010; Sander et al., 2011).

Another class of DNA-binding proteins are transcription activator-like effectors (TALES) that occur naturally in Xanthomonas bacteria (Boch et al., 2009; Moscou and Bogdanove, 2009). Individual TALE repeats recognize 1 nucleotide of DNA and assembly of individual repeats into larger arrays followed by fusion to Fokl resulted in TALE nucleases (TALENs) that could be used for site-specific genome editing (Christian et al., 2010; Miller et al., 2011). Generation and validation of TALENs is easier compared to ZFNs but their repetitive sequences render 
synthesis labor intensive and costly. Further, most of the TALE DNA-binding monomers are modular, but context-dependence of individual monomers can still alter their overall targeting specificity (Juillerat et al., 2014).

Next to customized nucleases that rely on DNA-binding proteins, the RNA-guided programmable nuclease Cas9 is based on DNA sequence recognition via Watson-Crick base pairing (Jinek et al., 2012). Cas9 is associated with the CRISPR system that functions as a bacterial adaptive immune system for antiviral defense (Barrangou et al., 2007). The ease of using Cas9 and its high efficiency for site-specific genome editing have led to wide adoptions and rapid advances in genome engineering applications within the last three years.

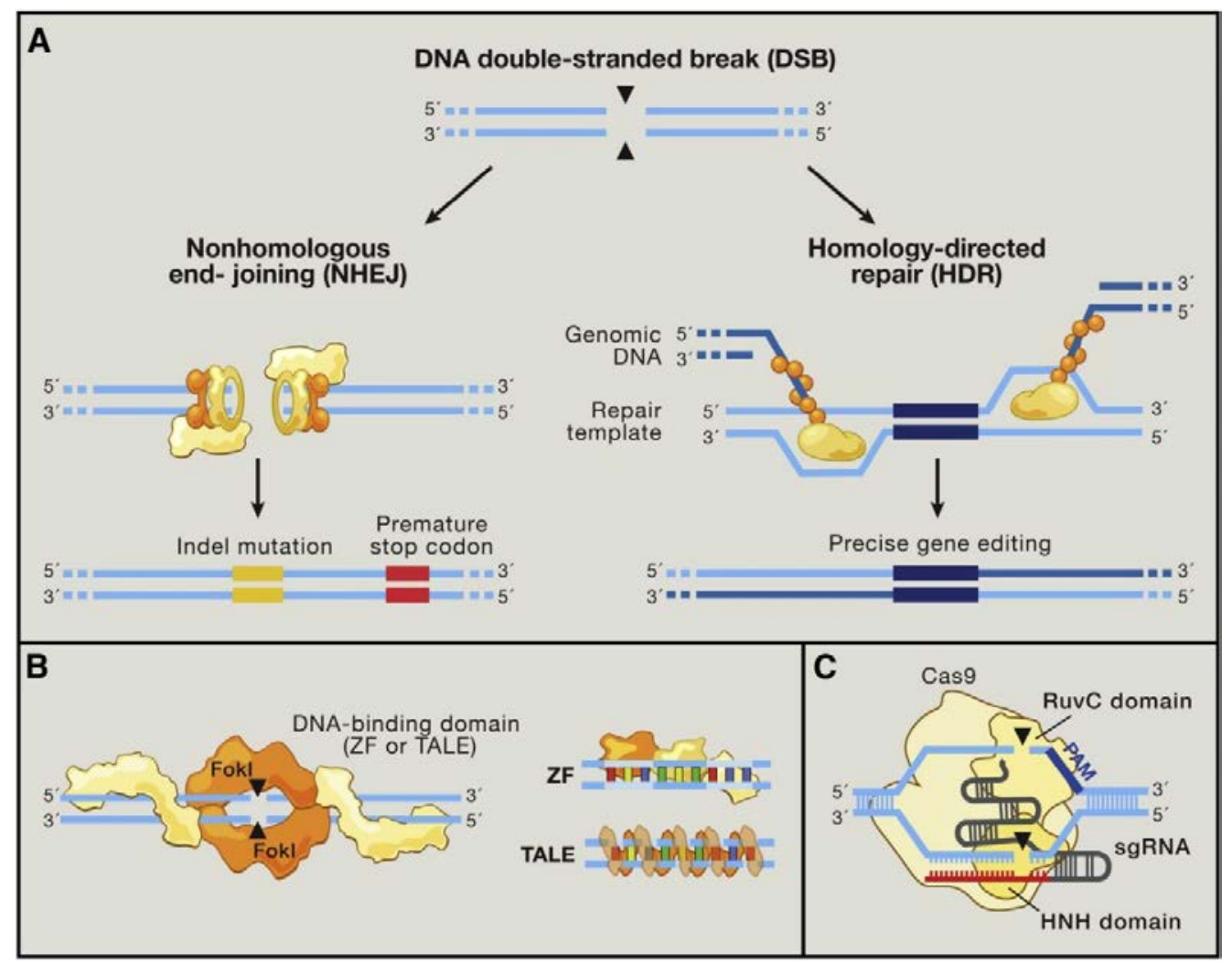

Figure 1: Site-specific nucleases as tools for genome engineering. a) Blunt DNA double-strand breaks (DSBs) are substrates for endogenous cellular DNA repair via nonhomologous end-joining (NHEJ) or homology-directed repair (HDR). The NHEJ pathway is error-prone and results in insertion/deletion mutations (indels) that lead to frameshifts and gene knockout. The HDR pathway leads to precise gene modifications via recombination with an exogenous homologous repair template. b) Zinc finger (ZF) proteins and transcription activator-like effectors (TALE) comprise DNA-binding domains that recognized 3 and $1 \mathrm{bp}$ of DNA, respectively. Modular assembly of ZFs or TALEs followed by fusion to Fokl endonuclease generates programmable site-specific nucleases. C) Cas9 is targeted to a specific DNA sequence via a single guide RNA (sgRNA) that undergoes Watson-Crick base-pairing with the DNA target. Cas9 requires a protospacer-adjacent motif (PAM) downstream of the target site to induce a DSB catalyzed by RuvC and HNH nuclease domains. (Adapted from Hsu et al., 2014 with permission from Elsevier). 


\subsubsection{Microbial CRISPR systems}

Clustered regularly interspaced short palindromic repeats (CRISPR) and CRISPR-associated (Cas) proteins comprise the CRISPR-Cas system that serves as a prokaryotic adaptive immune system. The first CRISPRs had been described as a set of nucleotide (nt) repeats that were interspaced by nonrepetitive spacer sequences in the genome of $E$. coli (Ishino et al., 1987). Clustered repeats were found in $>40 \%$ of bacteria and $90 \%$ of archaea and Cas genes were identified as well conserved elements adjacent to the repeats (Mojica et al., 2000; Jansen et al., 2002). Interestingly, DNA repeat sequences were found to be of foreign genetic origin, CRISPR loci were shown to be transcribed and Cas genes were predicted to encode proteins with nuclease and helicase domains (Tang et al., 2002; Bolotin et al., 2005; Haft et al., 2005; Mojica et al., 2005). The natural role of CRISPR-Cas as an adaptive immune system that provides resistance to bacteriophages was demonstrated by infection experiments of bacteria with lytic phages (Barrangou et al., 2007).

The overall sequence of CRISPR-mediated adaptive immunity involves three steps (Figure 2) (Barrangou and Marraffini, 2014). In the first step, adaptation, new spacer sequences are integrated into the CRISPR array from exogenous DNA. Second, CRISPR arrays are transcribed and processed into small CRISPR RNAs (crRNAs) that contain a repeat sequence and a spacer sequence for targeting the invading DNA. Finally, Cas proteins are guided by crRNA to cleave exogenous nucleic acids at sites complementary to the crRNA spacer sequence (Brouns et al., 2008; Marraffini and Sontheimer, 2008). Based on DNA sequence analysis and use of distinct molecular mechanisms, CRISPR-Cas systems have been classified into three major types: type I, II and III (Makarova et al., 2011). Type I and type II CRISPR systems require the presence of protospacer adjacent motifs (PAMs) adjacent to the crRNA-target site of the invading DNA (Shah et al., 2013). A large complex of multiple Cas proteins is required for crRNA-guided targeting of for nucleic acids in type I and type III CRISPR systems (Brouns et al., 2008; Hale et al., 2009). In contrast, the type II CRISPR system requires only a single Cas protein for RNA-guided DNA recognition and cleavage, a vital property for engineering a simple RNA-programmable nuclease (Jinek et al., 2012; Gasiunas et al., 2012). 


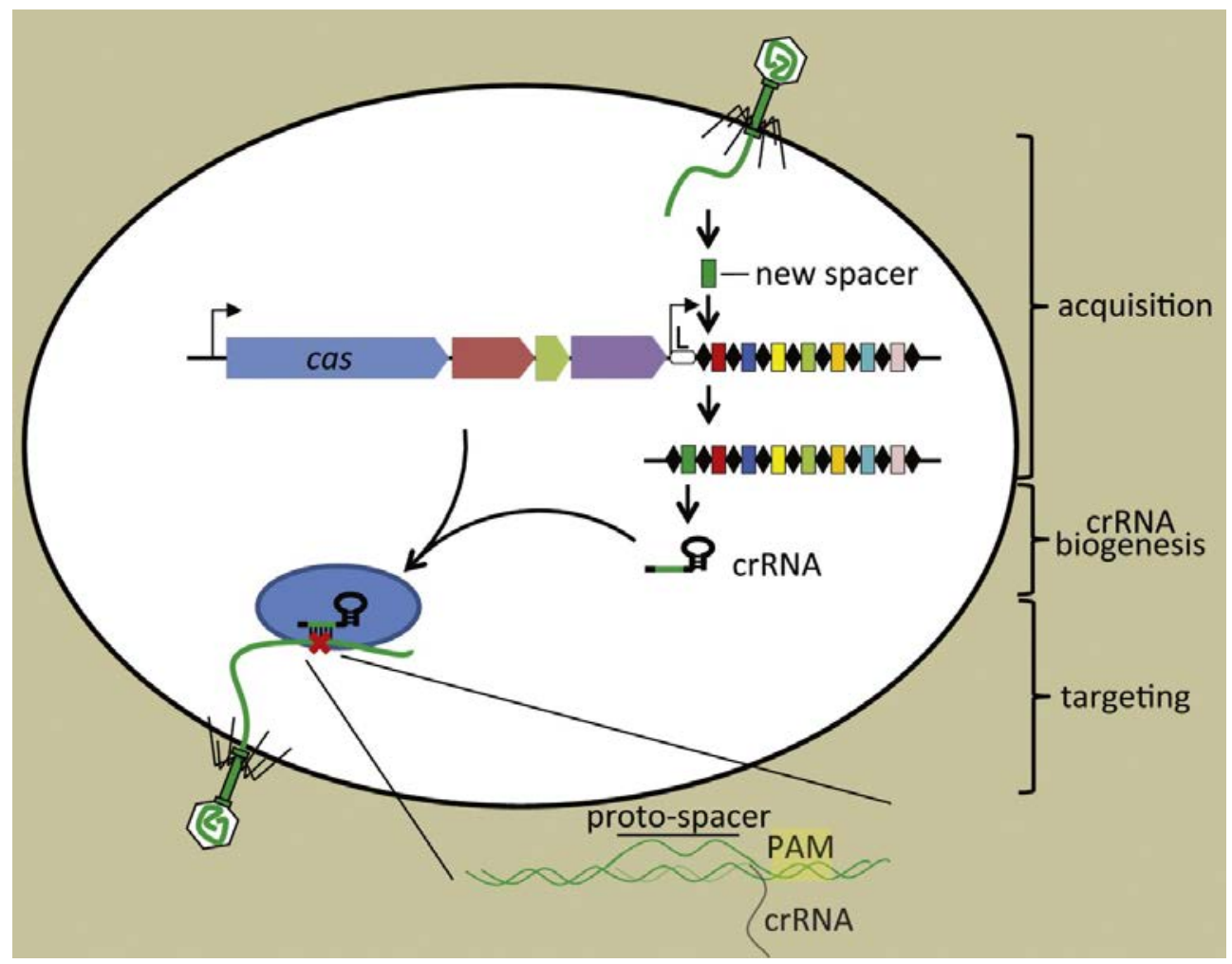

Figure 2: CRISPR-mediated immunity occurs in three stages. A typical CRISPR locus contains a set of repeat sequences (black diamonds) interspaced by nonrepetitive spacer sequences (colored boxes). Each CRISPR locus is flanked by a 'leader' sequence $(L)$ acting as a promoter for transcription and genes coding for CRISPR-associated (Cas) proteins. CRISPR-based immunity is established in three stages: (1) acquisition of spacer sequences from foreign phage DNA, (2) biogenesis of CRISPR RNA (crRNA) and (3) targeting of a DNA sequence complementary to the proto-spacer found in the crRNA (Adapted from Barrangou and Marraffini, 2014 with permission from Elsevier).

\subsubsection{The engineered CRISPR-Cas9 system}

The type II CRISPR locus of $S$. pyogenes contains a trans-activating crRNA (tracrRNA) that is essential for the processing of crRNAs by endoribunclease III and the CRISPR-associated protein Cas9 (Deltcheva et al., 2011). Cas9 functions as a dual RNA-guided endonuclease that could be programmed by a tracrRNA:crRNA duplex to direct DNA cleavage in vitro (Jinek et al., 2012). Moreover, a single guide RNA (gRNA) could be engineered by fusing a crRNA to a tracrRNA in which the target site of Cas9 was determined by the 20 nucleotide sequence at the $5^{\prime}$ end of the gRNA (Figure 3) (Jinek et al., 2012). Genome editing in mammalian cells was accomplished by heterologous expression of tracrRNA:crRNA hybrids as well as single gRNAs that direct Cas9 to induce site-specific DSBs in vivo (Cong et al., 2013; Jinek et al., 2013; Mali et al., 2013). Moreover, efficient multiplex genome editing can be accomplished by coexpression of several gRNAs together with Cas9 for simultaneous cleavage of multiple DNA target sites (Cong et al., 2013; Mali et al., 2013). Overall, the engineered CRISPR-Cas9 
system constitutes a powerful tool that uses a single gRNA to target any 20-bp DNA sequence flanked by an adjacent 5'-NGG PAM site. Unlike ZFNs and TALENS, CRISPR does not require complex protein engineering and the ease of Cas9 customization have led to a rapid adoption of CRISPR-Cas9 for genome editing purposes in a multitude of model systems (Doudna and Charpentier, 2014; Hsu et al., 2014).
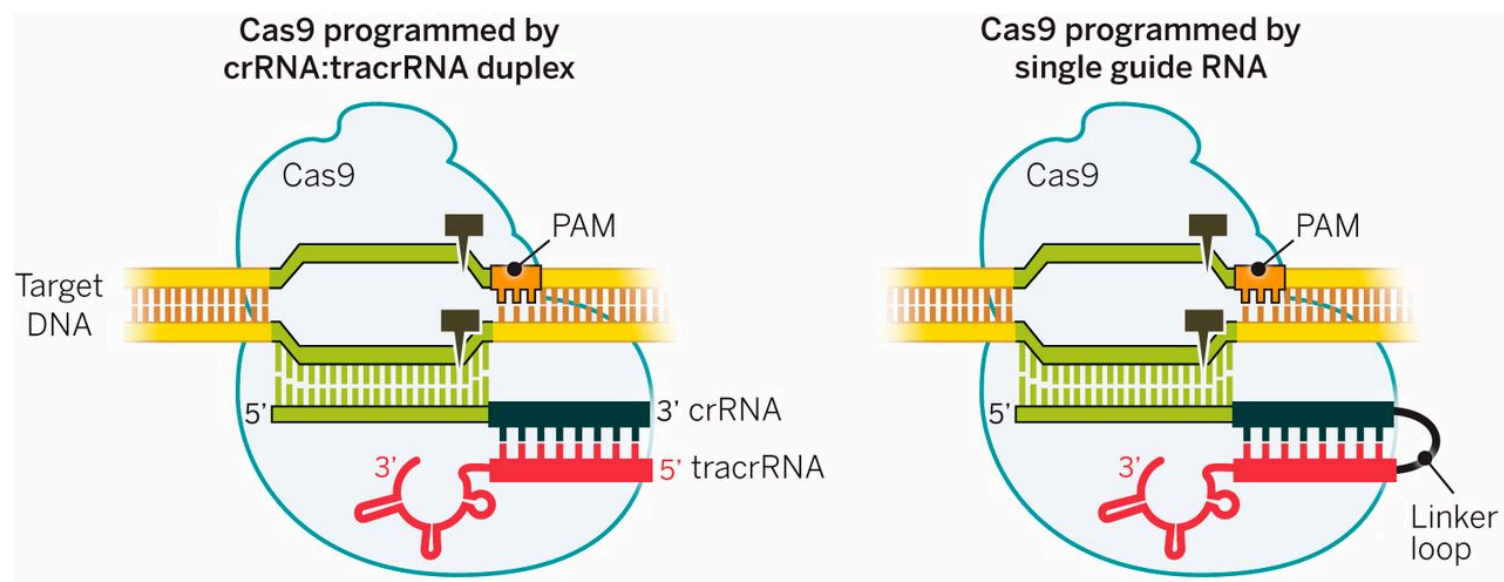

Figure 3: S. pyogenes Cas9 is a dual-RNA guided DNA endonuclease. Programming of Cas9 to a target site is mediated by crRNA:tracrRNA duplex or a single guide RNA containing both RNAs that are fused to each other via a linker loop. Any 20 nucleotide long DNA sequence can be targeted provided it contains an adjacent protospacer adjacent motif (PAM) with the sequence 5'-NGG. (Adapted from Doudna and Charpentier, 2014 with permission from AAAS).

\subsubsection{Determinants of Cas9 specificity}

Next to site-specific cleavage at target sites, Cas9 might be able to induce DSBs at unintended genomic 'off-target' sites. Initial studies suggested that the gRNA 'seed region' comprising the first PAM-proximal 8-12 nucleotides determines Cas9 specificity (Jinek et al., 2012; Cong et al., 2013). However, Cas9 exhibits a mismatch-tolerance along the entire gRNA sequence that depends on the number, position and distribution of mismatches (Fu et al., 2013; Hsu et al., 2013; Mali et al., 2013; Pattanayak et al., 2013). Moreover, mismatches depend on the chromatin structure and GC-content of target sites, are better tolerated at high Cas9 concentrations and limiting the duration of Cas9 expression influences the frequency of any DNA modification (Hsu et al., 2013; Dow et al., 2015b). Chromatin immunoprecipitation studies using catalytically inactive Cas9 demonstrated that Cas9 has many more binding sites than those matching the gRNA sequence (Kuscu et al., 2014; Wu et al., 2014b). Interestingly, active Cas9 was rarely observed to induce DNA cleavage at off-target binding sites indicating that both binding and cleavage require extensive gRNA:DNA pairing for efficient cleavage 
(Wu et al., 2014b; Sternberg et al., 2014). Further, genome-wide analysis of Cas9 specificity demonstrated a moderate activity on the majority of DNA sequences that differ from the gRNA sequence by two to three nucleotides (Tsai et al., 2015; Frock et al., 2015; Kim et al., 2015; Wang et al., 2015). Inefficient cleavage was still found when off-target and gRNA sequences differed by six nucleotides and some gRNAs acted on $>100$ off-target sites (Tsai et al., 2015). As off-target mutagenesis has the potential to cause local mutations as well as large DNA rearrangements, a variety of effective attempts have led to improved Cas9 specificities (Figure 4).

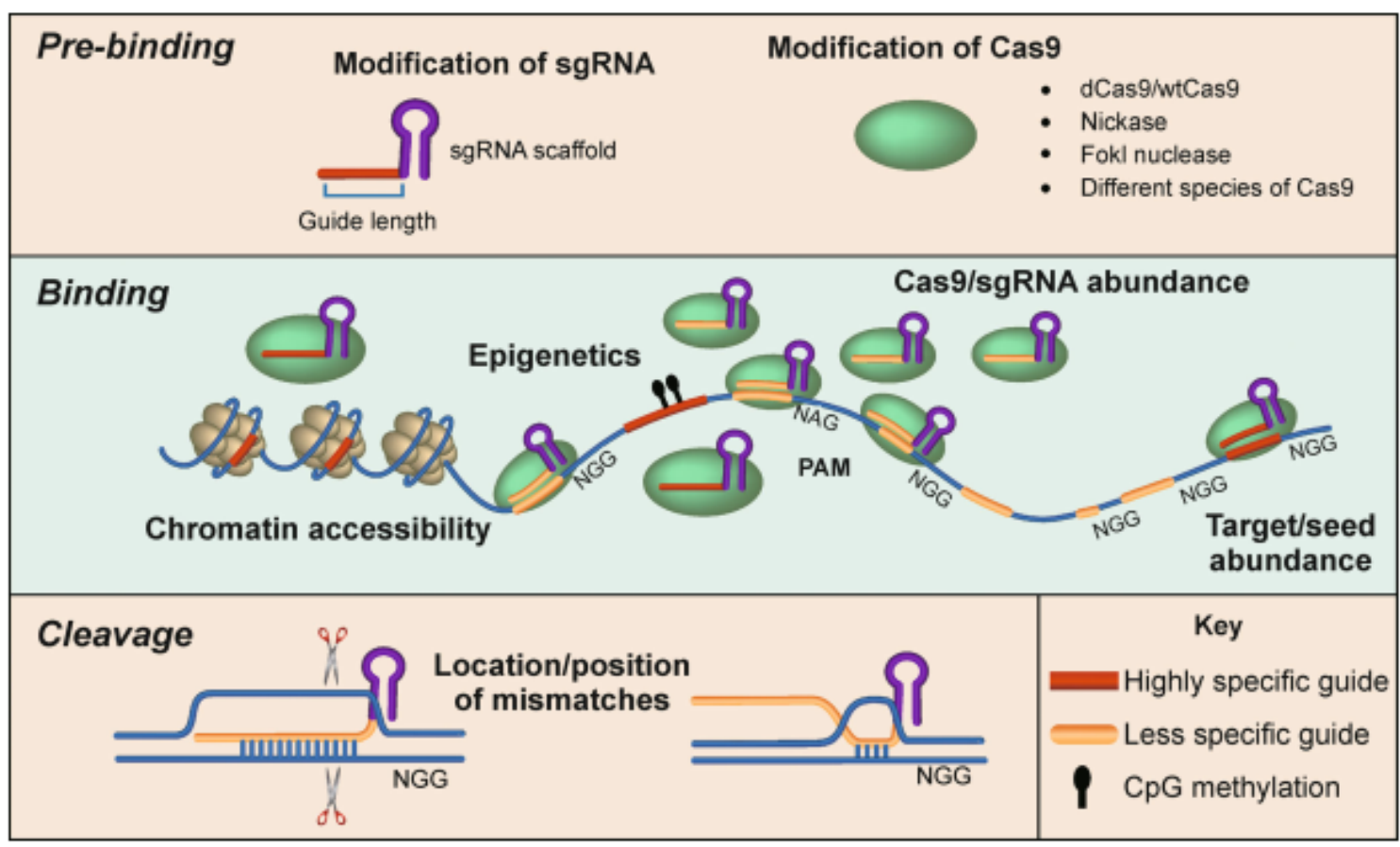

Figure 4: Determinants of Cas9 specificity. Top: Modifications of the single guide (sgRNA) or Cas9 itself can be used to increase specificity. Middle: S. pyogenes Cas9 requires a NGG or NAG PAM site for binding. Cas9 binding is negatively affected by closed chromatin and methylated DNA whereas binding of Cas9 is increase at high Cas9/sgRNA concentration and high abundance of similar target/seed sequences in the genome. Bottom: A short DNA sequence complementary to the seed region of the sgRNA already induces transient binding of Cas9. However, cleavage only occurs at DNA sequences with extensive sgRNA complementarity (Adapted from Wu et al., 2014a with permission from Springer).

First and foremost, judicious gRNA design is required in order to obtain high on-target efficiencies while reducing the number of unintended target sites. Computational tools based on experimental studies consider a number of potential gRNAs and can be applied for offtarget site predictions (Jamal et al., 2015). 
Lowering the effective concentration of the Cas9/gRNA complex by titrating down the amount of plasmid DNA in transfected cells was shown to improve Cas9 specificity albeit at decreased on-target efficiencies (Hsu et al., 2013). Limiting the duration of Cas9 expression or regulating Cas 9 activity with light provides precise spatial and temporal control over gene modifications (Dow et al., 2015b; Nihongaki et al., 2015; Polstein and Gersbach, 2015). The abundance of gRNAs can be tuned by expression from RNA Pol II promoters and processed from introns and ribozymes (Nissim et al., 2014; Kiani et al., 2014). Moreover, direct delivery of recombinant Cas9 together with in vitro transcribed gRNA was shown to reduce off-target mutagenesis in mammalian cells (Kim et al., 2014; Ramakrishna et al., 2014).

Cas9 nucleases induce a blunt-ended DSB catalyzed by the activity of their RuvC and $\mathrm{HNH}$ nuclease domains. Introduction of point mutation in one of the two nuclease domains converts Cas9 into a 'nickase' that creates a single-stranded break (DSB) (Jinek et al., 2012; Gasiunas et al., 2012). A pair of two nickases can be targeted to two nearby opposite strands and create a DSB that is efficiently repaired via NHEJ or HR (Mali et al., 2013; Ran et al., 2013a). Compared to wildtype Cas9, an improved specificity by up to 1,500-fold was demonstrated using the paired nickase approach (Ran et al., 2013a).

Truncation of gRNAs ('tru-gRNAs') to a length of 17 or 18 nucleotides showed a significant increase in Cas9 targeting specificity potentially caused by a higher mismatch sensitivity (Fu et al., 2014). Combination of tru-gRNAs with the paired nickase approach further reduced offtarget cleavage rates (Wyvekens et al., 2015).

A fusion of catalytically inactive Cas9 (dCas9) to Fokl nuclease monomers generated an RNA-guided nuclease that only induces DNA cleavage upon binding of two nearby gRNAs, which allows Fokl dimerization (Guilinger et al., 2014; Tsai et al., 2014). An increased specificity of at least 4-fold was reported when using dCas9-Fokl over a paired nickase (Guilinger et al., 2014; Tsai et al., 2014).

\subsubsection{Applications of CRISPR-Cas9}

Cas9-mediated genome editing was adapted for a wide range of applications in a variety of cells and model organisms (Figure 5). First studies showed that Cas9 could be used to facilitate targeted genome engineering in bacteria, human cancer and pluripotent stem cells in culture as well as zebrafish (Jiang et al., 2013; Cong et al., 2013; Jinek et al., 2013; Mali et al., 2013; Cho et al., 2013; Hwang et al., 2013). Subsequently, Cas9 enabled rapid genome alteration in yeast, fruit flies, mice and a multitude of other genetically tractable model organisms (Sander and Joung, 2014). 


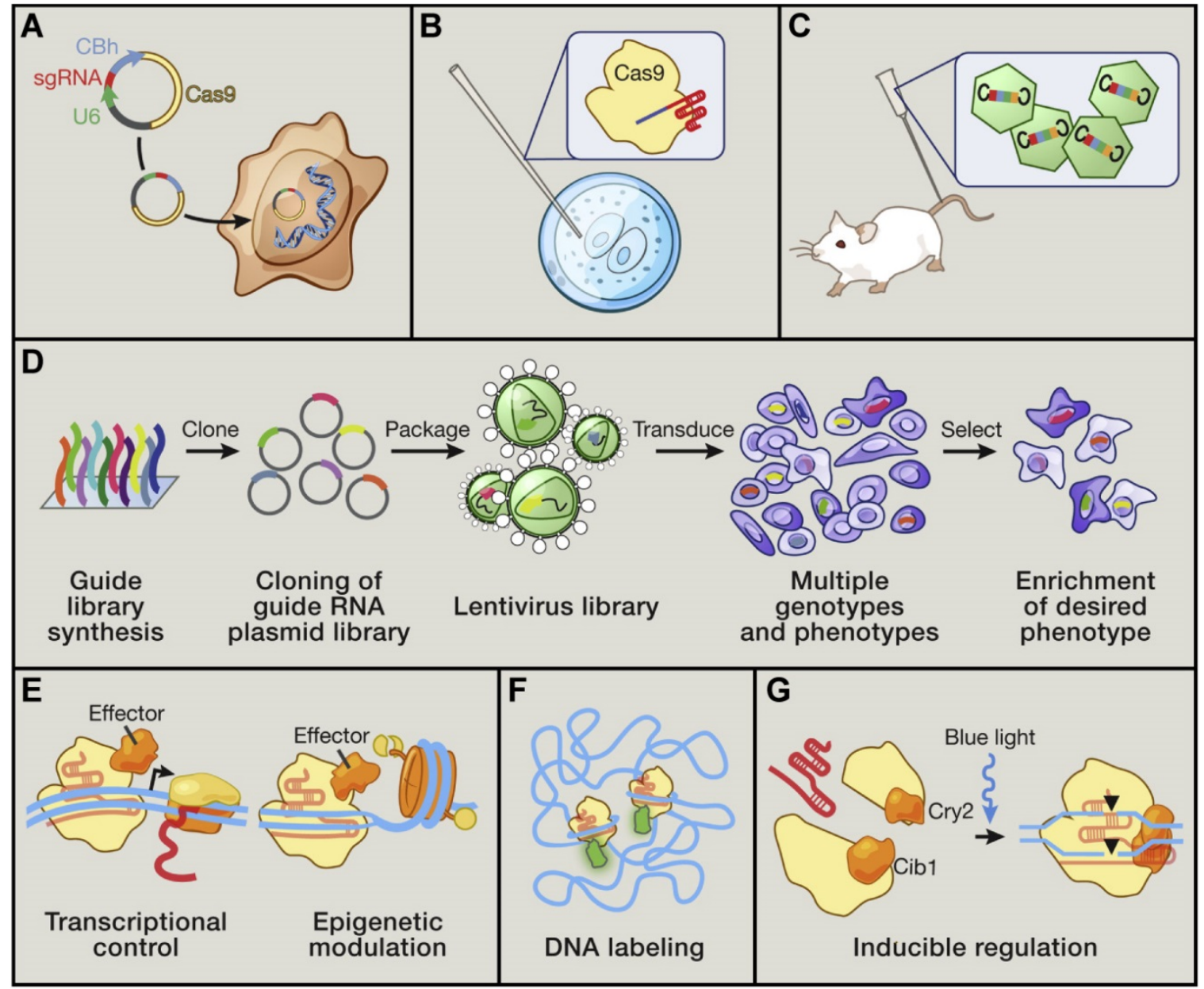

Figure 5: Application of CRISPR-Cas9. a) Genome editing in cultured cells by transfection of Cas9/gRNA expression plasmids. b) Generation of transgenic animals by microinjection of Cas9 protein and gRNA into fertilized zygotes. c) Somatic genome editing in adult animals by transduction of cells or tissues with CRISPR viral vectors. d) Genome-wide functional screens using gRNA libraries. e) Transcriptional control or epigenetic modulation based on catalytically dead Cas9 (dCas) fused to the respective effectors. f) Live cell DNA imaging using Cas9 fused to fluorescent reporters. g) Controlled Cas9 activity via optical or chemical induction. (Adapted from Hsu et al., 2014 with permission from Elsevier).

The simplicity of Cas9-mediated genome editing and its capability to modify many DNA target sites in parallel enabled genome-wide functional screens based on large gRNA libraries (Wang et al., 2014a; Shalem et al., 2014; Koike-Yusa et al., 2014). Using array-based oligonucleotide synthesis, libraries that contain between $\sim 64,000$ and $\sim 87,000$ distinct gRNAs have been constructed. After lentiviral delivery of gRNAs directed against early, constitutive exons of all human genes, loss-of-function screens based on positive and negative selection could be successfully performed. Compared to a partial knockdown achieved with RNAi 
libraries, Cas9-based approaches generate knockout cells and can be targeted to nearly any DNA sequence including non-coding regions (Shalem et al., 2014).

CRISPR interference (CRISPRi) can specifically repress transcriptional elongation by targeting of catalytically dead Cas9 (dCas9) to DNA sites in E. coli (Qi et al., 2013). Fusion of dCas9 to transcriptional repressor domains that promote gene silencing can further enhance the repressive activity in human cells (Gilbert et al., 2013). Moreover, conversion of Cas9 into an artificial transcriptional activator can be accomplished by fusing dCas 9 to activation domains (Konermann et al., 2013; Maeder et al., 2013; Perez-Pinera et al., 2013). Next to Cas9-mediated transcriptional modulation, locus-specific epigenome editing was achieved by fusion of epigenetic effectors to dCas9 (Hilton et al., 2015; Thakore et al., 2015).

Visualization of DNA in living cells was accomplished when EGFP-tagged dCas9 was specifically recruited to genomic loci (Chen et al., 2013). Robust imaging of repetitive DNA elements using a single gRNA as well as visualization of nonrepetitive DNA sequences using an array of gRNAs along a target locus was demonstrated.

Finally, the expression of Cas9 can be controlled in a temporal manner using an inducible CRISPR (iCRISPR) system (Dow et al., 2015a). Moreover, the 'split-Cas9' system provides precise spatial and temporal control over Cas9 activity using small molecules or light (Zetsche et al., 2015; Nihongaki et al., 2015).

In conclusion, the RNA-guided Cas9 nuclease derived from the prokaryotic CRISPR system has revolutionized our ability to precisely modify the genomes of diverse organisms. Multiple methodologies based on Cas9 allow a wide range of applications to control the organization, regulation and behavior of cells and organisms. While these developments took place just within the last three years, it will be exciting to see the future impact of CRISPR-Cas9 ranging from basic science to clinical applications.

\subsection{Fluorescent labelling and nanoscopy}

\subsubsection{Fluorescent proteins}

Fluorescence imaging of living cells enables the real-time observation of specific subcellular dynamics at high spatial and temporal resolution. Visualization of a protein of interest within a biological system mainly relies on the use of a genetically encoded fluorescent tag fused in frame to the specific protein. The most widely used probes in modern cell biology are fluorescent proteins (FP) that form bright and stable chromophores with a variety of spectral properties (Chudakov et al., 2010). 
The first FP was green fluorescent proteins (GFP) purified from the bioluminescent jellyfish A. victoria (Shimomura et al., 1962). GFP could be cloned and used for heterologous expression in E. coli and C. elegans without losing its fluorescent properties (Prasher et al., 1992; Chalfie et al., 1994; Inouye and Tsuji, 1994). GFP and its derivatives are about 240 amino acid residues long and fold into a barrel structure that contains eleven $\beta$-sheets with a molecular weight of about 27 kDa (Figure 6) (Ormo et al., 1996; Yang et al., 1996). Interestingly, the GFP chromophore forms spontaneously from only three amino acid residues in the presence of oxygen without the need for any further cofactors (Heim et al., 1994). An internal $\alpha$-helix contains the chromophore that is shielded from the bulk solvent by an extensive hydrogen-bonding network within the $\beta$-barrel. Importantly, the tertiary structure of all FPs has a great influence on chromophore formation and nearby amino acid residues play a crucial role for the photophysical properties of each FP variant (Shaner et al., 2005).

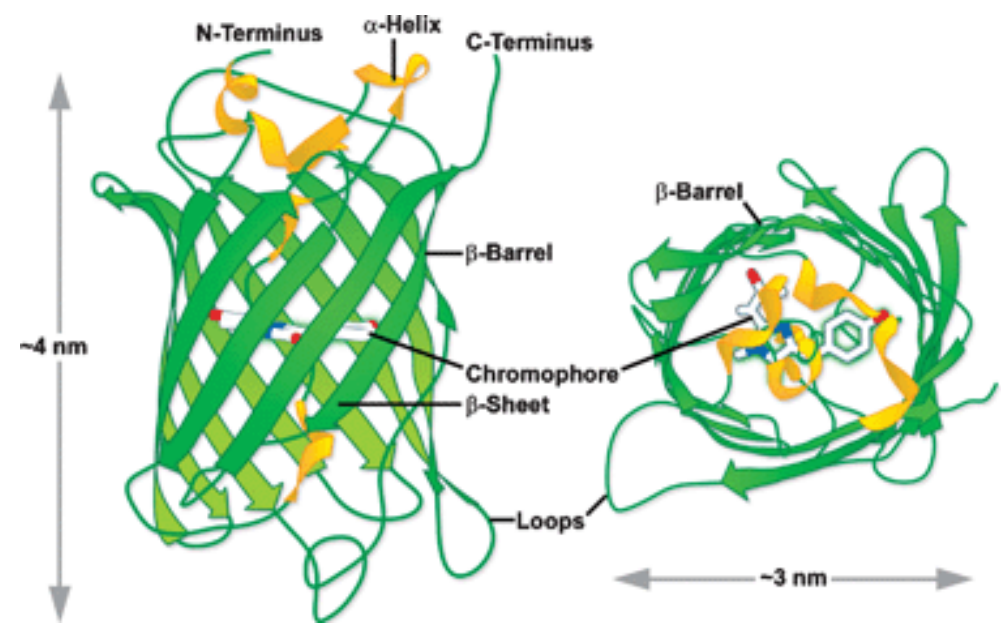

Figure 6: General structure of fluorescent proteins. Based on the crystal structure of $A$. victoria GFP the typical $\beta$-barrel architecture of all fluorescent proteins is exemplified. (Adapted from Day and Davidson, 2009 with permission of The Royal Society of Chemistry).

A variety of GFP-like proteins have been discovered from sea organisms that together with protein engineering efforts led to a vast number of FPs exhibiting different colors, brightness, photostability and tendency to oligomerize (Day and Davidson, 2009). Moreover, some FPs exhibit particular photophysical properties including photoactivation, photoconversion and photoswitching (Dean and Palmer, 2014). These are particularly useful properties that allow advanced imaging studies including protein tracking and movement, optical protein control and super-resolution microscopy applications (Nienhaus and Nienhaus, 2014). 


\subsubsection{Reversibly switchable fluorescent proteins}

Reversibly switchable fluorescent proteins (RSFPs) are FPs that can be repeatedly photoswitched between a non-fluorescent and a fluorescent state. The first RSFP used for in vivo photolabeling and protein tracking was 'kindling fluorescent protein' (KFP1) engineered from asFP595 (Chudakov et al., 2003). Initially KFP1 is not fluorescent, but green light illumination switches the protein into a fluorescent 'on-state' and results in red fluorescence. Irradiation with blue light leads to a reversible transition into a dark 'off-state'. However, the low quantum yield and the tetrameric nature of asFP595 and its derivatives including KFP1 limited their use for cell biology applications. The monomeric green RSFP 'Dronpa' was engineered from a tetrameric green coral FP (Ando et al., 2004). Initially Dronpa resides in a fluorescent on-state and blue light illumination induces off-switching accompanied by green fluorescence. This transition is reversible upon UV light illumination, which brings Dronpa back into its initial on-state. The switching modes exhibited by KFP1 and Dronpa are generally referred to as 'positive' and 'negative' switching (Figure 7) (Andresen et al., 2008). Yet another switching mode is found in the RSFP 'Dreiklang' (Brakemann et al., 2011). In the on-state, Dreiklang can be excited to emit green fluorescence using light of $511 \mathrm{~nm}$ while it can be switched off using $405 \mathrm{~nm}$ light. Reversible on-switching can be induced by $365 \mathrm{~nm}$ light illumination. Compared to positive or negative switchers, in which fluorescence emission and switching are coupled, Dreiklang shows a 'decoupled' switching mode.

RSFPs form a classical GFP-like $\beta$-barrel structure that encloses the autocatalytically formed 4-( $p$-hydroxybenzylidene)-5-imidazolinone ( $p$-HBI) chromophore. The molecular mechanism underlying photoswitching was clarified using X-ray crystallography studies on reversibly switchable protein crystals of asFP595 and Dronpa (Andresen et al., 2005; Andresen et al., 2007). Light irradiation results in a cis-trans isomerization of the chromophore methylene bridge, which is accompanied by complex structural rearrangements. The chromophore adopts a cis configuration, which corresponds to the fluorescent state while the trans isomer is associated to the non-fluorescent state. The isomerization state of the chromophore under equilibrium conditions is determined by stabilizing interactions between the chromophore and surrounding amino acid residues. Further, side chains that sterically influence the isomerization process govern the speed of reversible switching.

The unusual decoupled switching mechanism in Dreiklang is based on a reversible lightinduced hydration-dehydration reaction of the imidazolinone ring in the chromophore (Brakemann et al., 2011). The on-state chromophore exhibits two absorption peaks at 405 and $511 \mathrm{~nm}$. Irradiation with $405 \mathrm{~nm}$ light induces a covalent modification (hydration) of the 
imidazolinone ring that results in a nonfluorescent chromophore absorbing at $340 \mathrm{~nm}$. Illumination with $340 \mathrm{~nm}$ light induces a dehydration reaction at the off-state chromophore converting it back into the on-state.

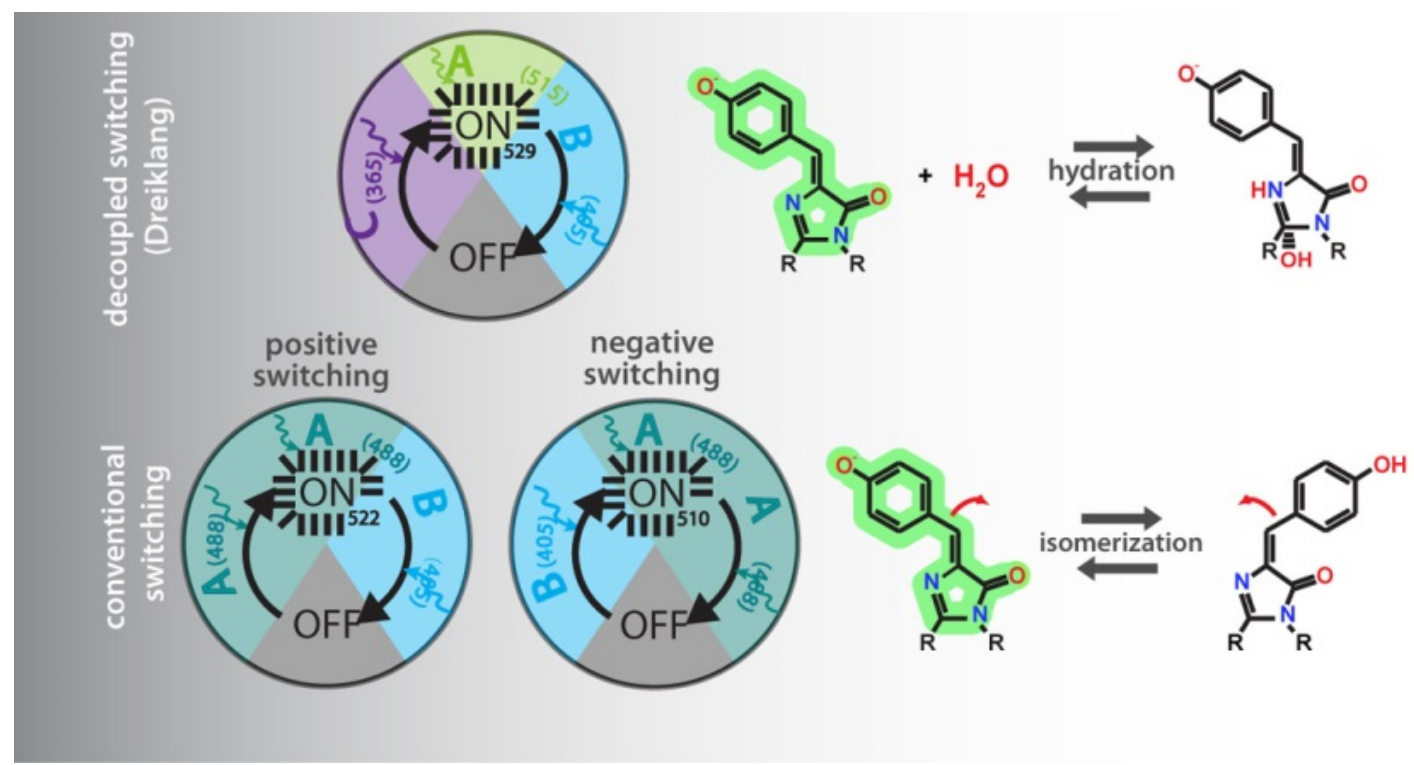

Figure 7: Switching mechanisms in reversibly switchable fluorescent proteins. Top: Dreiklang exhibits a decoupled switching mechanism. Under equilibrium conditions, fluorescent light is emitted upon irradiation with $515 \mathrm{~nm}$ light. Off-switching using $405 \mathrm{~nm}$ light induces chromophore hydration, while on-switching mediated by $365 \mathrm{~nm}$ light leads to dehydration of the chromophore. Bottom: Switching is based on cis-trans isomerization of the chromophore and coupled to fluorescence excitation in all other RSFPs. Positive switcher: $488 \mathrm{~nm}$ excitation light switches the RSFP to the onstate and $405 \mathrm{~nm}$ switches it to the off-state. Negative switcher: $488 \mathrm{~nm}$ excitation light induces offswitching, while $405 \mathrm{~nm}$ is used for off-switching.

\subsubsection{Live cell super-resolution with RSFPS}

Live cell fluorescence microscopy allows unprecedented insights into dynamic processes of living cells, tissues and whole organisms. However, the resolution of a conventional fluorescence microscope is limited by diffraction to about N/2NA (about $200 \mathrm{~nm}$ ) in the focal plane with $\lambda$ and NA denoting the wavelength and the numerical aperture of the used objective lens, respectively (Pawley, 2013). Visualization of structures below this diffraction limit could so far only be realized using electron microscopy (EM), which is incompatible with living cells. To overcome the diffraction limit, a number of super-resolution microscopy concepts were developed that achieve nanoscale resolution down to $20 \mathrm{~nm}$ in living systems (Hell, 2009). These concepts are based on reversible photoswitching of fluorophores between a bright state and a dark state and can be grouped into two categories (Figure 8). 
a)
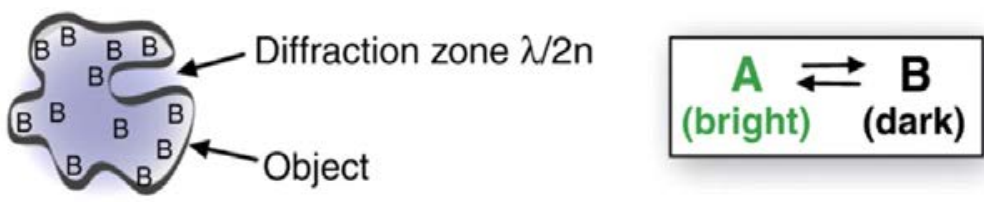

b)
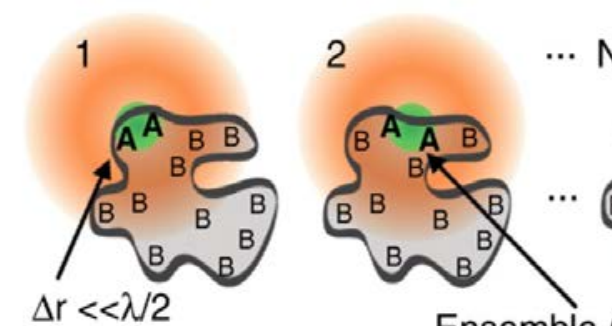

$\cdots \mathrm{N}$

Targeted

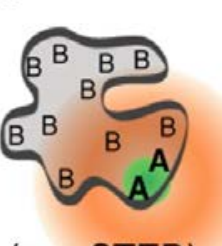

Ensemble (e.g. STED)

c)
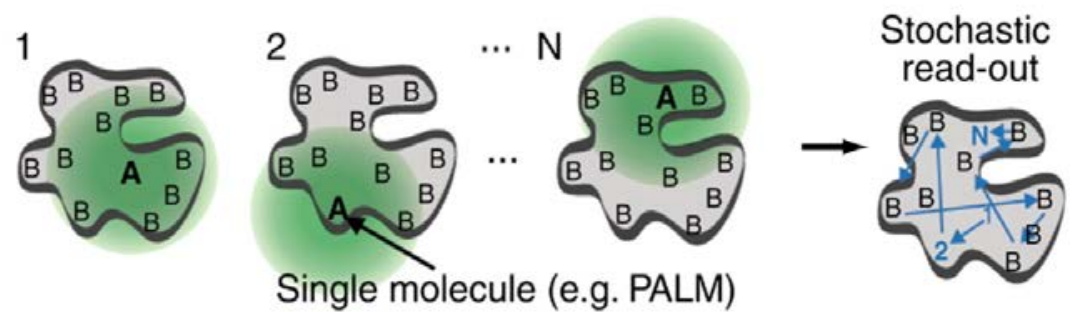

Figure 8: Basic principles of super-resolution microscopy. a) Displayed is a nanostructured object within the diffraction zone that exhibits a minimal size of $\lambda / 2 N A$. The object is labelled with fluorophores that can reversibly switch between a bright state (A) or a dark state (B). b) Targeted switching of an ensemble of molecules generates a subdiffraction-sized spot with bright molecules $(A)$ while switching peripheral molecules into a dark state (B). Scanning of the sample point-by-point yields a superresolved image. C) Stochastic methods employ single fluorophore switching at random positions to a bright state $(A)$ while other molecules remain dark (B). The localization of the bright molecule can be calculated from the centroid of the fluorescence spot with nanoscale precision (Adapted from Hell, 2007 with permission from AAAS).

Stochastic approaches (PALM/STORM) rely on temporal switching of a subset of individual fluorophores (Betzig et al., 2006; Rust et al., 2006; Hess et al., 2006). Weak sample illumination sparsely switches individual fluorophores that are further apart than N2NA to a bright state from which they emit fluorescent photons. Detection of $N \gg 1$ photons from a diffraction spot enables calculation of its centroid with a localization precision depending on the number of N. Subsequently these fluorophores are switched off to a non-fluorescent state enabling localization of a new set fluorophores that reside at other positions. The cycle of activation, detection and deactivation is repeated in order to reconstruct a super-resolved image from the localization of individual molecules.

Deterministic approaches (RESOLFT/STED) utilize targeted switching of an ensemble of molecules (Hell, 2007). The RESOLFT (reversible saturable optical fluorescence transitions) principle is based on the insight that converting a subset of fluorophores into a non- 
fluorescent dark state can substantially decrease the effective fluorescent region. Practically, RESOLFT achieves sub-diffraction resolution by (1) exciting fluorophores using a diffractionlimited light beam, (2) switching off fluorophores at the periphery using a donut shaped beam and (3) reading out the remaining fluorophores. Point-by-point scanning of the three beams across the entire sample generates a RESOLFT super-resolution image that exhibits nanoscale resolution. Compared to conventional diffraction-limited fluorescence microscopy, RESOLFT is diffraction-unlimited and the attainable resolution is given by:

$$
d=\frac{\lambda}{2 N A \sqrt{1+\frac{I_{\max }}{I_{S}}}}
$$

with $\lambda, N A, I_{\max }$ and $I_{\mathrm{s}}$ denoting the wavelength of light, the numerical aperture of the objective lens, the maximum intensity of the donut beam and the saturation intensity of the used fluorophore, respectively (Hell, 2007). Hence, the attainable resolution of a RESOLFT microscope is given by the value of $I_{\max } / I_{\mathrm{S}}$ and is technically 'infinite' with $I_{\max } / I_{\mathrm{S}} \rightarrow \infty$. In practice, resolution is determined by the actual choice of the on- and off-state and the saturation intensity $I_{S}$ required to drive a transition between both states.

The first concept of the RESOLFT type was STED (stimulated emission depletion) microscopy which employs an electronic transition between the excited fluorescent state $S_{1}$ and the ground state $S_{0}$ (Hell and Wichmann, 1994; Klar et al., 2000). STED uses a focused excitation beam and a red-shifted donut-shaped beam for quenching excited fluorophores via stimulated emission from $S_{1} \rightarrow S_{0}$. Because the fluorescence lifetime of the excited state is short (ns), high saturation intensities in the order of $10^{9} \mathrm{~W} / \mathrm{cm}^{2}$ are required. This ultimately translates into high depletion intensities in the range of $10^{9}-10^{12} \mathrm{~W} / \mathrm{cm}^{2}$ required to obtain a super-resolved image.

The second RESOLFT type of concept is GSD (ground state depletion) microscopy, which utilizes the electronic transition between the singlet system $\left(S_{1}\right.$ plus $\left.S_{0}\right)$ and the metastable triplet state $T_{1}$ (Hell and Kroug, 1995). To switch off fluorescence in the periphery of the excitation spot, GSD employs an excitation laser to transiently transfer fluorophores to the triplet state $T_{1}$. As the lifetime of the triplet state is in the range of $\mu s-m s$, the saturation intensity $I_{S}$ is reduced accordingly. The dye can be switches off at $10^{3}-10^{6}$ times lower intensities than with STED to obtain a similar resolution using this mechanism. 
RESOLFT with RSFPs employs a photochemical transition of the chromophore between a fluorescent and a non-fluorescent state (Hell et al., 2003; Hofmann et al., 2005). Photoswitching between two long lived chemical states of a chromophore requires a low saturation intensity $I_{S}$ that ultimately results in an ultralow depletion intensity $I_{\text {max }}$ ranging from W/cm² - kW/cm² (Hell, 2003; Hofmann et al., 2005). RESOLFT is particularly suited for live cell imaging because it uses low light intensities similar to those used in confocal microscopy while achieving nanoscale resolution. Moreover, RESOLFT employs genetically encoded RSFPs that may be introduced to a variety of model systems including cultured cells, tissues and whole organisms.

RESOLFT relies on bright and photostable RSFPs that can undergo a high amount of switching cycles and exhibit a high contrast between the on- and off-state. Further, for live cell imaging applications those RSFPs should exhibit fast switching kinetics and be monomers that mature efficiently at $37^{\circ} \mathrm{C}$. While the first RSFPs including KFP1 or Dronpa exist as tetramers or show a low number of switching cycles, an array of RSFPs suitable for RESOLFT imaging has been developed over the last few years. Among those is rsEGFP that can be reversibly photoswitched more than 1,000 times and enabled live cell RESOLFT imaging of bacteria and mammalian cells imaged at <40-nanometer resolution (Grotjohann et al., 2011). The uncoupled switching mechanism of Dreiklang enabled super-resolution microscopy based on targeted and stochastic switching (Brakemann et al., 2011). Mutagenesis of rsEGFP led to the generation of rsEGFP2 and rsEGFP(S205N) that exhibit useful features for imaging cellular dynamics and parallelized RESOLFT nanoscopy, respectively (Grotjohann et al., 2012; Chmyrov et al., 2013). Moreover, the Dronpa variant DronpaM159T was successfully applied for imaging morphological changes deep inside living brain slices (Testa et al., 2012). Finally, dual-color live cell RESOLFT nanoscopy was realized recently by coexpression of two RSFPs in mammalian cells and neurons (Lavoie-Cardinal et al., 2014; Testa et al., 2015).

\subsection{Prohibitin and Mitochondrial biology}

\subsubsection{Mitochondrial structure and dynamics}

Mitochondria are double membrane organelles of endosymbiotic origin in eukaryotic cells. An essential feature of mitochondria is their ability to efficiently produce ATP via oxidative phosphorylation (OXPHOS). Additionally mitochondria are the major source of NADH and are central to many biochemical pathways such as $\beta$-oxidation of fatty acids, pyrimidine and 
phospholipid metabolism, heme synthesis and others (Nunnari and Suomalainen, 2012). Moreover, mitochondria are key players in diverse cellular processes such as calcium ion storage, regulation of apoptosis, autophagy and cellular differentiation (Kasahara and Scorrano, 2014).

The two mitochondrial membranes separate the organelle into four distinct compartments: outer mitochondrial membrane (OMM), inner mitochondrial membrane (IM), intermembrane space (IMS) and the matrix (Figure 9) (Palade, 1952; Sjostrand, 1953). Import of mitochondrial precursors occurs through the translocase of the outer membrane (TOM) complex that closely cooperates with the translocase of the inner membrane (TIM) complex and other transport complexes for proper protein sorting (Dudek et al., 2013). The mitochondrial matrix harbors many biosynthetic enzymes and contains the mitochondrial DNA (mtDNA) while the IMS serves as a transport hub between the cytoplasm and the matrix (Herrmann and Riemer, 2010). The mitochondrial IM can be further subdivided into the highly folded cristae membrane harboring OXPHOS complexes and the inner boundary membrane (IBM) that is opposed to the OM and connected to cristae via cristae junctions (CJ).

Mitochondria are highly dynamic organelles that undergo frequent morphology changes caused by fusion and fission processes (Westermann, 2010). Mitochondrial morphology reflects the physiological state of the cell as the organelle appears fragmented during apoptosis or elongated during starvation (Suen et al., 2008; Gomes et al., 2011). 'Mitochondria-shaping' proteins exert regulation and maintenance of mitochondrial morphology. OMM fusion requires the dynamin-related GTPases Mitofusion (Mfn) 1 and 2 that reside in the OMM and form homo- and heterodimers to affect fusion in a GTP. dependent manner (Koshiba et al., 2004). Fusion of the IMM is controlled by Optic Atrophy 1 (OPA1) that is localized in the IM but faces the IMS where it controls IMM fusion in coordination with Mfn1 (Cipolat et al., 2004). Mitochondrial fission is mediated by the cytosolic soluble dynamin-related protein 1 (DRP1) (Smirnova et al., 2001). Upon translocation from the cytoplasm to mitochondria, DRP1 interacts with OM adaptor proteins to oligomerize and form spiral filaments that lead to constriction of the mitochondrial membranes (Mears et al., 2011). While the conserved core components of the fusion-fission machinery are known, mechanistic insights about their assembly and regulation in the context of other factors including cytoskeletal proteins, membrane contact sites and further mitochondrial proteins is lacking (Kasahara and Scorrano, 2014). 
a
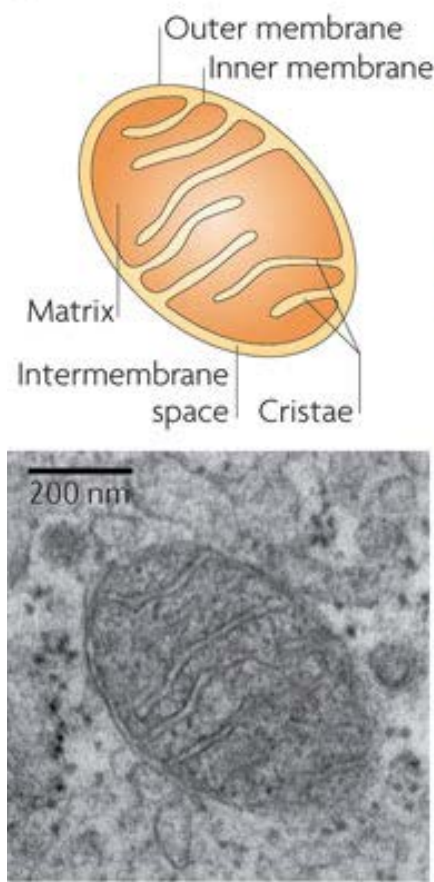

b

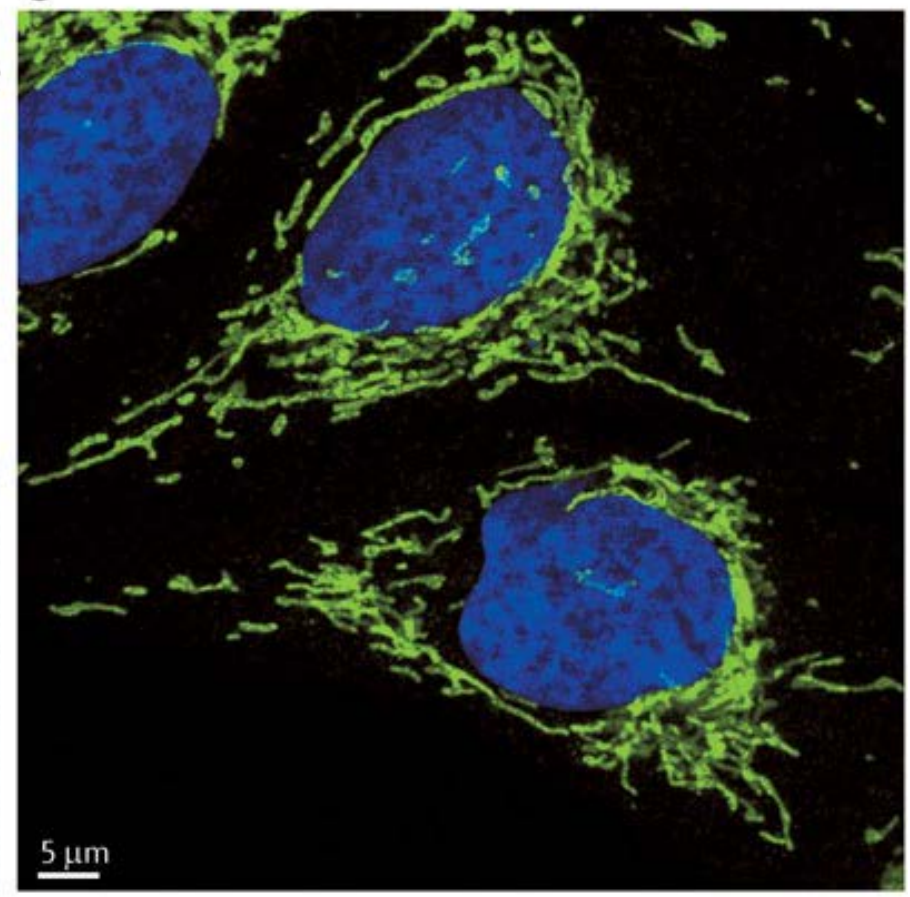

Figure 9: Structure of mitochondria. a) Top: Schematic of a typical mitochondrion that comprises four compartments: outer membrane, inner membrane, intermembrane space and matrix. The inner membrane forms characteristic folds termed cristae. Bottom: Transmission electron microscopy image of mitochondria of human fibroblast cells in ultrathin sections. b) Mitochondria (green) form tubular structures that spread throughout the entire cytoplasm in many eukaryotic cells. Mitochondrial of human osteosarcoma (U2OS) cells were stained by indirect immunofluorescence using an anti-TOM20 antibody for confocal microscopy. Nuclei (blue) were stained with DAPI (4',6-diamidino-2-phenylindole). (Adapted from Westermann, 2010 with permission from Macmillan Publishers Ltd).

\subsubsection{The mitochondrial prohibitin complex}

Prohibitins denote a family of conserved eukaryotic proteins with the two homologous subunits prohibitin 1 (PHB1) and prohibitin 2 (PHB2). The PHB1 gene product was discovered as an inhibitor of cell proliferation although this activity could be solely attributed to the 3' untranslated region of the gene (McClung et al., 1989; Jupe et al., 1996). PHB2 was isolated in association with PHB1 as an interaction partner of the IgM antigen receptor and both proteins were also named B-cell-receptor complex-associated proteins (BAP32 = PHB1 and BAP37 = PHB2) (Terashima et al., 1994). Independent of PHB1, PHB2 was identified as a repressor of nuclear estrogen receptor activity and termed REA (Montano et al., 1999).

Human PHB1 has a molecular weight of $30 \mathrm{kDa}$ while human PHB2 is a $33 \mathrm{kDa}$ protein (Figure 10). Both prohibitins contain unconventional non-cleavable signal sequences at their N-terminus that ensure mitochondrial import and sorting the IMM (Tatsuta et al., 2005; Kasashima et al., 2006). Hydropohobic N-terminal regions are required for membrane anchoring while the C-terminal region is facing the IMS (Berger and Yaffe, 1998; Tatsuta et 
al., 2005). Further, the C-terminus harbors the so-called PHB domain which is characteristic for proteins of the SPFH (stomatin/prohibitin/flotillin/HflK)-family of membrane proteins (Browman et al., 2007).

PHB1 and PHB2 interact to form a stable macromolecular PHB complex of 1-1.4 MDa at the IM of yeast, mammals and C. elegans (Steglich et al., 1999; Nijtmans et al., 2000; Artal-Sanz et al., 2003). Homodimers or PHB1 and PHB2 have been not detected and crosslinking studies suggest that PHB1 and PHB2 bind to each other in a heterodimeric fashion (Back et al., 2002; Tatsuta et al., 2005). It has been proposed that the yeast PHB complex contains about 12-16 (Back et al., 2002) or 16-20 (Tatsuta et al., 2005) heterodimers that associate to form a ring-like structure at the IMM with a diameter of 20-25 nm. Interestingly, prohibitins are functionally interdependent and depletion of either PHB1 or PHB2 leads to loss of the remaining prohibitin and absence of the PHB complex (Merkwirth et al., 2008; Artal-Sanz et al., 2003; Berger and Yaffe, 1998).

A

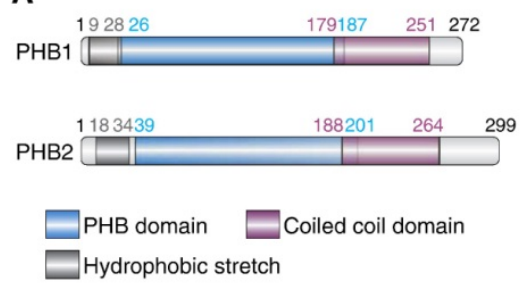

B

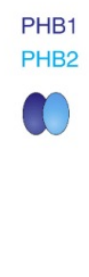

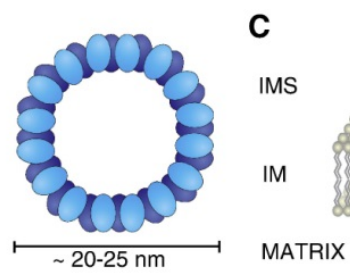

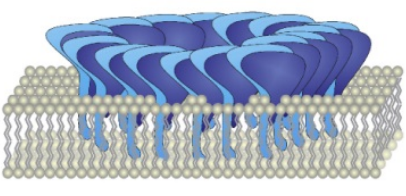

Figure 10: Prohibitins form a mitochondrial membrane complex. a) Domain structures of the mammalian prohibitins PHB1 and PHB2. b) Heterodimers of PHB1 and PHB2 form the building blocks for ring-like PHB complexes with a speculative stoichiometry and a diameter of 20-25 nm. c) N-terminal hydrophobic stretches anchor the PHB complex to the mitochondrial inner membrane (IM). C-terminal $\mathrm{PHB} / \mathrm{SPFH}$ and coiled-coil domains are exposed to the intermembrane space (IMS). (Adapted from Merkwirth and Langer, 2009 with permission from Elsevier)

\subsubsection{Functions of the mitochondrial PHB complex}

A diverse set of cellular functions including cell cycle progression, transcriptional regulation, cellular senescence, apoptosis and mitochondrial biogenesis have been attributed to prohibitins (Artal-Sanz and Tavernarakis, 2009; Merkwirth and Langer, 2009). Moreover, prohibitins were found to localize to different cellular compartments including the plasma membrane, the nucleus and mitochondria (Mishra et al., 2005; Mishra et al., 2006). However, recent studies in different model systems indicate that cellular homeostasis depends on prohibitin function and localization within the IMM (Merkwirth et al., 2008; Schleicher et al., 2008). 
Depletion of prohibitins in yeast or human cells as well as in C. elegans leads to highly fragmented mitochondria (Artal-Sanz et al., 2003; Berger and Yaffe, 1998; Osman et al., 2009b; Kasashima et al., 2006). Similarly, fragmented mitochondria accumulated in the absence of prohibitins in mouse embryonic fibroblasts (MEFs) or mouse embryonic stem (ES) cells (Merkwirth et al., 2008; Kowno et al., 2014). Ultrastructural analysis of prohibitindeficient MEFs revealed an absence of lamellar-shaped cristae or an accumulation of vesicular-shaped structures within mitochondria (Merkwirth et al., 2008). Absence of prohibitins resulted in the destabilization of OPA1, a large dynamin-like GTPase essential for mitochondrial fusion and cristae morphogenesis (Hoppins et al., 2007). Proteolytic processing of OPA1 splice variants generates long (L-OPA1) and short (S-OPA1) (Ishihara et al., 2006). Interestingly, absence of prohibitins in MEFs leads to selective loss of L-OPA1 isoforms and wildtype mitochondrial morphology could be restored cells by overexpression of a noncleavable OPA1 variant (Merkwirth et al., 2008). Because prohibitin depletion leads to an increased proteolytic activity of the m-AAA protease (Steglich et al., 1999), it was suggested that mammalian prohibitins control OPA1 processing indirectly via regulation of m-AAA protease activity (Merkwirth and Langer, 2009). However, experimental evidence for this hypothesis is lacking.

The mitochondrial inner membrane protein stomatin-like protein 2 (SLP-2) has been shown to directly associate with and stabilize prohibitins (Da Cruz et al., 2008). Prohibitins and SLP-2 are both members of the SPFH family of proteins and SLP-2 has been linked to mitochondrial fusion via interaction with the GTPase mitofusin 2 (Mfn2) (Browman et al., 2007; Hajek et al., 2007). A small portion of Mfn2 participates in Mfn2/SLP-2 heterooligomers and SLP-2 is required for mitochondrial hyperfusion under stress conditions (Hajek et al., 2007; Tondera et al., 2009). Interestingly, absence of SLP-2 leads to selective loss of L-OPA1 isoforms under stress, a phenotype that corresponds to prohibitin-depletion under normal conditions indicating that OPA1 processing is regulated by a complex network of similar proteins.

In the absence of prohibitins, genes that encode for proteins involved in the biosynthesis of phosphatidylethanolamine (PE) and cardiolipin (CL) become essential (Birner et al., 2003; Osman et al., 2009a). Decrease of PE or CL levels in prohibitin-deficient cells is detrimental and leads to distortions of the IMM that ultimately result in mitochondrial membrane dissipation and cell death (Osman et al., 2009a). Thus, the integrity of the IMM depends on prohibitins upon reduction of PE or CL levels.

The ring-sized PHB complex has been proposed to constitute a protein and lipid scaffold in the inner mitochondrial membrane required to define functional subcompartments (Figure 11) 
(Osman et al., 2009b). This model is supported by the sequence similarity between prohibitins and SPFH proteins that form large membrane assemblies and have been associated with lipid rafts (Huber et al., 2006; Browman et al., 2007). The formation of PHB complex protein scaffolds for lipid microdomains might explain the variety of phenotypes associated with prohibitin deficiency (Osman et al., 2009a). However, due to a lack of experimental tools, evidence for this model has to be awaited.
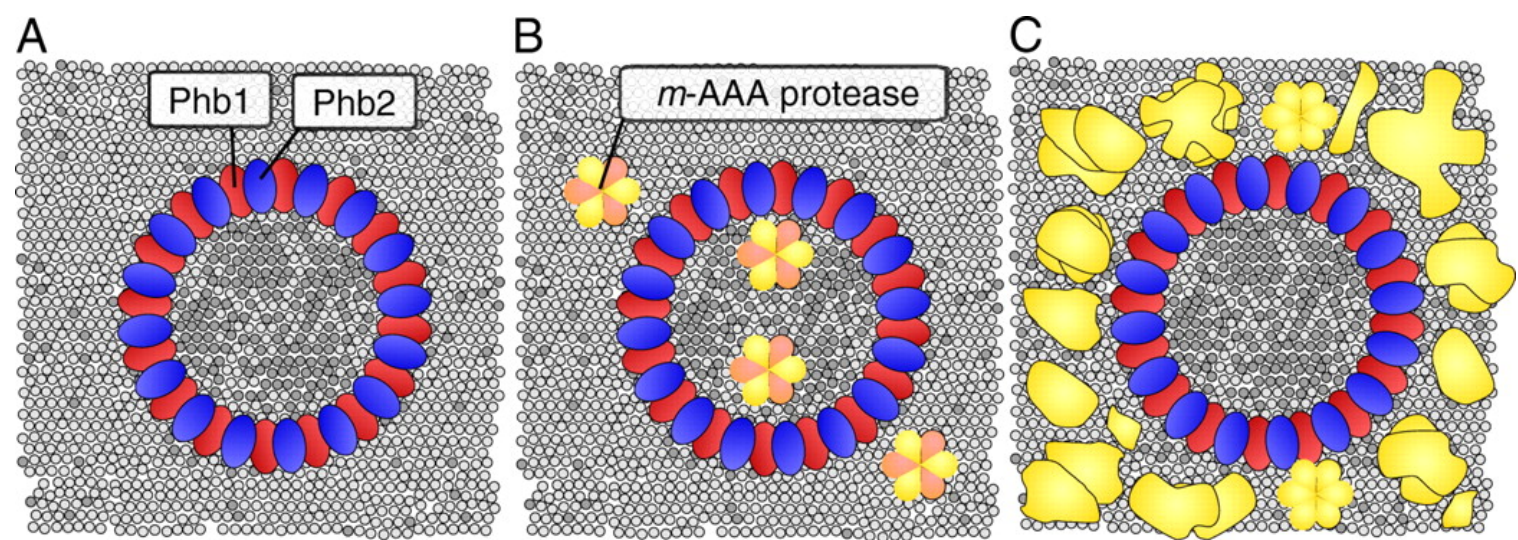

Figure 11: PHB complexes as putative membrane scaffolds. a) PHB complexes as lipid scaffolds support an asymmetrical distribution of phospholipids in the inner mitochondrial membrane (IMM). Cardiolipin and/or phosphatidylethanolamine (dark grey circles) might be enriched in the interior of each PHB ring. b) PHB complexes as protein scaffolds to recruit specific proteins such as m-AAA protease to distinct functional sites. c) PHB complexes with a fence-like function exclude membrane proteins (yellow) from specific areas to generate protein-free lipid patches with functional relevance. (Adapted from Osman et al., 2009b with permission from The Company of Biologists Ltd).

\subsection{Aims}

RESOLFT super-resolution microscopy is the currently most suited method for nanoscale analysis of living systems due to the use of low light doses and the accompanied reduced phototoxic damage. Despite those benefits, RESOLFT relied on transient overexpression of fluorescently tagged proteins so far. To circumvent common problems associated with transient overexpression, the first goal of this thesis was the establishment of a workflow for endogenous fluorescent protein (FP) tagging in human cells using CRISPR-based genome engineering. This would, at the same time, extend the range of CRISPR applications as endogenous FP tagging in human cells was not reported so far. Moreover, whether typical artefacts observed in transient transfection experiments are caused by artificially high expression levels of fusion proteins or the fluorescent tag itself was not clear. Human cell lines that express a fluorescently tagged fusion protein would provide a suitable model 
system to investigate such issues. Finally, the compatibility of endogenously tagged cells as samples for live-cell RESOLFT analysis had to be addressed, because super-resolution imaging at native protein levels could allow quantitative nanoscale imaging, but its potential was not investigated so far.

Interestingly, while prohibitins have been studied for more than two decades, many fundamental aspects about their biological organization remain to be uncovered. Localization and dynamics could not be addressed at the beginning of this thesis, because high quality antibodies were lacking and prohibitin overexpression leads to non-physiological effects. Therefore, the established workflow for CRISPR-based gene editing had to be applied to generate human cells that express endogenously tagged PHB1 and PHB2. Further, it was not clear whether prohibitin tagging in human cells is possible and overexpression-induced artefacts could be avoided. Finally, prohibitin knock-in cells would provide an excellent model system to gain novel insights about their localization, dynamics and abundance in human cells. 


\section{Materials and Methods}

\subsection{Bacterial and human cell culture}

\subsubsection{E. coli strains and cultivation}

E. coli strains used for cloning and plasmid propagation were DH5 $\alpha$ and $\mathrm{DH} 10 \beta$ (Thermo Fisher Scientific, Waltham, MA, USA). For protein expression the BL21 E. coli strain was used (Stratagene, La Jolla, CA, USA).

Cultivation of $E$. coli cells was carried out in Luria Bertain (LB) medium ( $0.5 \%$ yeast extract $(\mathrm{w} / \mathrm{v}), 1 \%$ peptone $(\mathrm{w} / \mathrm{v}), 0.5 \% 1 \mathrm{~N} \mathrm{NaOH}(\mathrm{v} / \mathrm{v}), 0.5 \% \mathrm{NaCl}(\mathrm{w} / \mathrm{v})$ ) or on agar plates (LB medium supplemented with $2 \%(\mathrm{w} / \mathrm{v})$ agar) in the presence of selection antibiotics. The culture density of E. coli cells was measured with an Eppendorf BioPhotometer ${ }^{\circledR}$ (Eppendorf, Hamburg, Germany) in a plastic cuvette at OD600 with the respective medium as a reference. Selection antibiotics were ampicillin ( $50 \mu \mathrm{g} / \mathrm{ml}$; Sigma Aldrich, Munich, Germany) and kanamycin (50 $\mathrm{\mu g} / \mathrm{ml}$; Applichem, Darmstadt, Germany).

\subsubsection{E. coli transformation via electroporation}

Electrocompetent DH5 $\alpha$ and BL21 cells were generated according to a modified protocol initially described by Dower et al., 1988. An overnight preculture of the respective E. coli strain was used to inoculate $250 \mathrm{ml} \mathrm{LB}$ medium. The main culture was grown at $37^{\circ} \mathrm{C}$ until it reached an OD600 of about 0.6 followed by incubation at $4^{\circ} \mathrm{C}$ for $15 \mathrm{~min}$. After this point the cells were kept ice cold. The cells were harvested by centrifugation at $4000 \mathrm{~g}$ and $4^{\circ} \mathrm{C}$ for 15 min in a flat bottom centrifuge bottle. The supernatant was discarded and the pellet was resuspended in $250 \mathrm{ml}$ ice cold water, centrifuged again as above, and resuspended in $5 \mathrm{ml}$ $10 \%$ glycerol (1/50 volume of initial culture volume). The suspension was aliquoted to $40 \mu \mathrm{l}$ portions, flash frozen in liquid nitrogen and stored at $-80^{\circ} \mathrm{C}$.

For electroporation $40 \mu \mathrm{l}$ of electrocompetent cells were thawed on ice. Cells were transferred to an electroporation cuvette (2 mm gap width) and mixed with 5-25 $\mathrm{ng}$ of plasmid DNA. The mixture was incubated on ice for $5 \mathrm{~min}$ and electroporation was carried out at $2.5 \mathrm{kV}$. The cells were resuspended in $1 \mathrm{ml}$ fresh LB medium and incubated at $37^{\circ} \mathrm{C}$ for $1 \mathrm{~h}$ while shaking. Afterwards the cells were plated on agar plates supplemented with the respective selective antibiotic and incubated at $37^{\circ} \mathrm{C}$ overnight. 


\subsubsection{E. coli transformation via heat-shock}

Chemically competent $\mathrm{DH} 10 \beta$ cells for transformation via heat-shock were prepared according to Hanahan et al., 1991. A main culture was established by inoculating $250 \mathrm{ml}$ of LB medium with a drop of overnight preculture. The cells were grown at $37^{\circ} \mathrm{C}$ until an OD600 of about 0.3 was reached and harvested by centrifugation at $4000 \mathrm{~g}$ and $4^{\circ} \mathrm{C}$ for $15 \mathrm{~min}$ (Sorval RC-5B, DuPont Instruments, Wilmington, DE, USA) in a flat bottom centrifuge bottle. The supernatant was discarded and the pellet resuspended in $80 \mathrm{ml}$ of ice cold CCMB80 buffer (10 mM KOAc, 80 mM CaCl 2,20 mM MnCl 2,10 mM MgCl $2,10 \%$ (v/v) glycerol, pH 6.4) followed by incubation on ice for $20 \mathrm{~min}$. After another centrifugation step at $4^{\circ} \mathrm{C}$, the pellet was resuspended in $10 \mathrm{ml} \mathrm{CCMB80}$ and the suspension adjusted to a final OD600 of 1.0 using CCMB80. Aliquots of $100 \mu \mathrm{l}$ were flash frozen and stored at $-80^{\circ} \mathrm{C}$ for further use.

For transformation $50 \mu \mathrm{l}$ of chemically competent cells were thawed on ice and mixed with 10$50 \mathrm{ng}$ of plasmid DNA. After incubation on ice for $30 \mathrm{~min}$, the mixture was transferred to a $42^{\circ} \mathrm{C}$ preheated water bath or thermocycler for $30 \mathrm{sec}$. After an additional incubation on ice for $2 \mathrm{~min}, 200 \mu \mathrm{l}$ SOC medium was added and the cells incubated at $37^{\circ} \mathrm{C}$ for $1 \mathrm{~h}$ while shaking. The entire suspension was plated on agar plates supplemented with selective antibiotics at $37^{\circ} \mathrm{C}$ overnight.

\subsubsection{Plasmid isolation and verification}

Isolation of plasmid DNA from E. coli cells was based on the alkaline lysis method (Birnboim and Doly, 1979) using the QIAprep Spin MiniPrep or MidiPrep Kit (Qiagen, Hilden, Germany). Plasmid DNA was eluted in ultrapure water and the sequence verified using the Sanger sequencing (Sanger et al., 1977) service offered by Seqlab Göttingen.

\subsubsection{Cultivation of human cells}

U2OS and HeLa cells (American Type Culture Collection, Manassas, VA, USA) were cultured in Dulbecco's modified Eagle's medium (DMEM) (Invitrogen, Carlsbad, CA, USA) supplemented with $10 \%$ fetal bovine serum (PAA, Pasching, Austria), 100 units/ml penicillin, $100 \mu \mathrm{g} / \mathrm{ml}$ streptomycin (all Biochrom, Berlin, Germany), and $1 \mathrm{mM}$ sodium pyruvate (Sigma, St. Louis, MO, USA) under constant conditions at $37{ }^{\circ} \mathrm{C}$ and $5 \% \mathrm{CO}_{2}$. Splitting of confluent cells was done by detachment from the growth surface using 0.05\% Trypsin/ 0.02\% EDTA (Biochrom, Berlin, Germany) followed by transfer to fresh DMEM at a ratio of 1:3-1:6 every 23 days. Cells were used until passage number 30 . 


\subsubsection{Chemical transfection of human cells}

For transfection of mammalian cells FuGENE HD (Promega, Mannheim, Germany) was used. Therefore $2 \times 10^{5}$ U2OS cells or $3 \times 10^{5}$ HeLa cells were seeded per well of a 6-well plate 12-16 hours before transfection. The following day, transfection was carried out using a FuGENE $\mathrm{HD}$ reagent to DNA ratio of 3.5 to 1 and a total DNA amount of $3 \mu \mathrm{g}$ according to the manufacturer's protocol.

\subsubsection{Electroporation of human cells}

Electroporation of U2OS cells was done using Nucleofection ${ }^{\mathrm{TM}}$ (Lonza, Basel, Switzerland). Therefore cells were detached from the growth surface, counted using a Scepter ${ }^{\mathrm{TM}} 2.0$ Cell counter (EMD Millipore, Billerica, MA, USA) and harvested by centrifugation at $300 \times \mathrm{g}$ for 2 min. Resuspension of $1 \times 10^{6}$ cells was done using $100 \mu \mathrm{l}$ Nucleofector ${ }^{\mathrm{TM}}$ Solution $\mathrm{V}$. Cells were mixed with 5-10 $\mu \mathrm{g}$ plasmid DNA, transferred to a cuvette and transfected with a Nucleofector $^{\mathrm{TM}}$ device using program $\mathrm{X}-001$.

\subsubsection{Genomic DNA isolation from human cells}

Isolation of genomic DNA from U2OS or HeLa cells for genotyping via PCR was carried out with the DNeasy Blood \& Tissue Kit (Qiagen, Hilden, Germany). The DNA was concentration was determined using a NanoDrop1000 spectrophotometer (Thermo Fisher Scientific, Waltham, MA, USA) and diluted to $100 \mathrm{ng} / \mu \mathrm{l}$ with ultrapure water.

\subsubsection{Flow cytometry of human cells}

Fluorescence activated cell sorting (FACS) using a FACSAria II (BD Biosciences, Heidelberg, Germany) was applied to analyze expression levels of fusion proteins in cells transiently transfected or endogenously tagged. Further, FACS was used to isolate single cells after cotransfection of nuclease/donor plasmids. Fluorescence excitation of both rsEGFP2 and Dreiklang was done using an Argon laser at $488 \mathrm{~nm}$ and a 530/30 emission filter for detection.

\subsection{Molecular biology methods}

\subsubsection{Polymerase chain reaction (PCR)}

Amplification of defined DNA sequences from plasmid or human genomic DNA was done using polymerase chain reaction (PCR). Therefore $50 \mathrm{ng}$ (plasmid) or $100 \mathrm{ng}$ (genomic DNA) template DNA was mixed with $1 \mu$ forward primer and $1 \mu$ reverse primer (10 $\mu \mathrm{M}$ each), $1 \mu \mathrm{l}$ 
dNTP solution (2 mM of each: dATP, dGTP, dCTP, dTTP), $10 \mu$ of $5 \times$ Q5 $^{\circledR}$ reaction buffer (NEB, Ipswich, MA, USA) and $1 \mu \mathrm{l} 5^{\circledR}$ DNA polymerase (2 U/ $\mu$ l) (NEB, Ipswich, MA, USA). The mixture was filled up to $50 \mu$ with sterile water. DNA amplification was carried out with a TPersonal Thermocycler (Biometra, Göttingen, Germany) according to the following PCR program:

\begin{tabular}{ll}
\hline Temperature $\left[{ }^{\circ} \mathrm{C}\right]$ & Time \\
\hline 95 & $5 \mathrm{~min}$ \\
\hline 95 & $20 \mathrm{sec}$ \\
$55-65$ & $20 \mathrm{sec}$ \\
72 & $15 \mathrm{sec} / 1 \mathrm{~kb}$ \\
\hline 95 & $5 \mathrm{~min}$ \\
\hline
\end{tabular}

\subsubsection{Agarose gel electrophoresis}

Agarose was dissolved in TAE buffer (40mM Tris, 20mM acetic acid, 1mM EDTA pH 8.0) by boiling in a microwave. Analytical or preparative agarose gels were poured in custom made gel trays. Samples were mixed with the required amount of $6 x$ loading buffer $(30 \%(v / v)$ glycerol, $0.25 \%(\mathrm{w} / \mathrm{v})$ bromophenol blue, $0.25 \%(\mathrm{w} / \mathrm{v})$ xylene cyanol) and loaded to the gel together with a Gene Ruler 100 bp or 1 kb ladder (Thermo Fisher Scientific, Waltham, MA, USA). The gel was run at $100 \mathrm{~V}$ for $20 \mathrm{~min}$ and stained in $0.5 \mu \mathrm{g} / \mathrm{ml}$ ethidium bromide solution for 10-30 min.

\subsubsection{TBE-PAGE}

Fragment analysis of DNA was done using TBE-PAGE (Tris-Borate-EDTA polyacrylamide gel electrophoresis). Samples were mixed with 6x loading buffer (30\% (v/v) glycerol, 0.25\% (w/v) bromophenol blue, $0.25 \%(\mathrm{w} / \mathrm{v})$ xylene cyanol) and loaded to a $10 \%$ Mini-PROTEAN $^{\circledR}$ TBE Precast Gel (Bio-Rad, Munich, Germany) placed in a Mini- PROTEAN ${ }^{\circledR}$ Tetra Cell (Bio-Rad, Munich, Germany) filled with 1x TBE buffer (89 mM Tris, 89 mM Boric Acid, 2 mM EDTA, pH 8.3). GeneRuler 100 bp DNA ladder (Thermo Fisher Scientific, Waltham, MA, USA) was used as a reference for DNA sizing. For electrophoretic DNA separation a current of $150 \mathrm{~mA}$ per gel was applied until the bromophenol blue marker reached the bottom of the gel. Gels were stained in $0.5 \mu \mathrm{g} / \mathrm{ml}$ ethidium bromide solution for $30 \mathrm{~min}$ followed by a destain step in water for $20 \mathrm{~min}$. 


\subsubsection{Gibson Assembly}

Gibson Assembly (Gibson et al., 2009) was used for isothermal one-step assembly of multiple DNA fragments. Primers with complementary overhangs were designed using the NEBuilder ${ }^{\circledR}$ Assembly Tool v1.9 (NEB, Ipswich, MA, USA). PCR fragments were amplified using Q5 ${ }^{\circledR}$ high fidelity DNA polymerase (NEB, Ipswich, MA, USA) and purified using a MinElute PCR purification kit (Qiagen, Hilden, Germany). Linearized plasmid was prepared by restriction digestion with EcoRV in $1 \times$ CutSmart $^{\circledR}$ buffer (NEB, Ipswich, MA, USA) and dephosphorylation using $1 \cup$ FastAP (Thermo Fisher Scientific, Waltham, MA, USA) followed by purification using gel extraction (Qiagen, Hilden, Germany). 50 ng of each DNA were mixed on ice with $1 \mu \mathrm{l}$ of 10x Gibson Assembly Master Mix (Qiagen, Hilden, Germany) and filled up to $10 \mu \mathrm{l}$ with water. The mixture was incubated at $50^{\circ} \mathrm{C}$ in a thermocycler for $1 \mathrm{~h}$ and $2 \mu \mathrm{l}$ were used for transformation of chemically competent $E$. coli DH10 $\beta$ cells via heat-shock. Positive clones were screened via colony PCR.

\subsubsection{Colony PCR}

Identification of $E$. coli colonies that contain the desired plasmid after transformation was done using colony PCR. Therefore $24 \mu$ of CloneID ${ }^{\mathrm{TM}}$ 1x Colony PCR Master Mix (Lucigen, Middleton, WI, USA) were mixed with $0.5 \mu$ forward primer and $0.5 \mu$ l reverse primer $(10 \mu \mathrm{M}$ each) specific for the construct of interest. A bacterial colony was replicated on a LB agar plate containing the required selective antibiotic followed by transfer of the colony to $25 \mu$ of PCR mix. DNA amplification was carried out with a TPersonal Thermocycler (Biometra, Göttingen, Germany) according to the following program:

\begin{tabular}{ll}
\hline Temperature $\left[{ }^{\circ} \mathrm{C}\right]$ & Time \\
\hline 95 & $5 \mathrm{~min}$ \\
\hline 95 & $20 \mathrm{sec}$ \\
57 & $20 \mathrm{sec}$ \\
72 & $1 \mathrm{~min} / 1 \mathrm{~kb}$ \\
\hline 95 & $5 \mathrm{~min}$ \\
\hline
\end{tabular}

\subsubsection{Cloning of overexpression plasmids}

Cloning of rsEGFP2 or Dreiklang fusion proteins for plasmid-driven overexpression in mammalian cells was carried out using the primers listed in Table 4. The rsEGFP2 or Dreiklang (DK) coding sequence was amplified from plasmid rsEGFP2-MAP2 (Stefan Jakobs lab, ID: p716) or from plasmid DK-Tubulin (Stefan Jakobs lab, ID: p3031), respectively. VIM 
cDNA was amplified from the plasmid pmKate2-vimentin (Evrogen, Moscow, Russia). The following cDNAs were amplified from pDONR223 plasmids obtained from the hORFeome V7.1 collection: HMGA1 (ID: 4996); ZYX (ID: 4546); PHB1 (ID: 6030) and PHB2 (ID: 394). The respective fragment combinations (rsEGFP2 with VIM, ZYX or HMGA1; DK with PHB1 or PHB2) were mixed with EcoRV-digested, dephosphorylated pFLAG-CMV-5.1 (Sigma Aldrich, St. Louis, MO, USA) for Gibson Assembly as described above.

\subsubsection{Design and cloning of donor plasmids}

All primers used for rsEGFP2 or Dreiklang (DK) donor plasmid cloning are listed in Table 5. DNA sequences for left homology arm (LHA) and right homology arm (RHA) were amplified from human genomic DNA. The rsEGFP2 or DK coding sequence was amplified from plasmid rsEGFP2-MAP2 (Stefan Jakobs lab, ID: p716) or from plasmid DK-Tubulin (Stefan Jakobs lab, ID: p3031), respectively.

Donor plasmid design was done in silico using SeqBuilder of the Lasergene 12 package (DNASTAR, Madison, WI, USA). The length of each homology arms was between 500 bp and 1000 bp. Both LHA and RHA were designed such that commonly used restriction sites between each homology arm and the transgene are be generated. This allows simple exchange of the respective transgene at later stages. The plasmid backbone was pUC57 plasmid (Thermo Fisher Scientific, Waltham, MA, USA). Primer design for Gibson Assembly of the donor plasmids HMGA1-rsEGFP2, ZYX-rsEGFP2, PHB1-DK, and PHB2-DK was done using the NEBuilder ${ }^{\circledR}$ Assembly Tool v1.9 (NEB, Ipswich, MA, USA). Primer design for standard cloning of VIM-rsEGFP2 was done manually.

For the VIM-rsEGFP2 donor plasmid, PCR products were purified, digested with Kpnl/Notl (LHA), Notl/Ncol (rsEGFP2), Ncol/Sall (RHA) and cloned into a pUC57 plasmid that was digested with Kpnl/Sall by a standard four fragments ligation. For HMGA1-rsEGFP2, ZYXrSEGFP2, PHB1-DK and PHB2-DK the three respective PCR products were purified and cloned into a pUC57 using Gibson Assembly as described above. Silent mutations within the ZYX-rsEGFP2 donor corresponding to the terminal exon of the ZYX gene were introduced by including a gBlock ${ }^{\circledR}$ DNA fragment (IDT, San Jose, CA, USA) that contained the desired modifications into the Gibson Assembly mixture. Silent mutations within the respective Cas9 binding region were introduced using the QuikChange II site-directed mutagenesis kit (Agilent Technologies, CA, USA) and the primers listed in Table 5. 


\subsubsection{Design and cloning of nuclease plasmids}

Sequence information about each target site within the human genome was obtained from the Gene database provided by the National Center for Biotechnology information (http://www.ncbi.nlm.nih.gov/gene). Design of each guide RNA (gRNA) was carried out using the CRISPR Design Tool (Hsu et al., 2013; http://crispr.mit.edu). Bicistronic pX330 expression vector expressing Cas9 and gRNA (Cong et al., 2013) was digested with Bbsl (NEB, Ipswich, MA, USA) and gel-purified (Qiagen, Hilden, Germany). Oligonucleotide pairs (Table 6) were hybridized and ligated into the Bbsl-digested pX330. The plasmid pX330 was obtained from the Addgene plasmid repository (Addgene, Cambridge, MA, USA).

\subsubsection{Guide RNA in vitro cleavage assay}

A T7 promoter was added to pX330-based guide RNA (gRNA) templates by PCR amplification using primers listed in Table 7. The T7-gRNA product was purified using a MinElute Kit (Qiagen, Hilden, Germany) and used as a template for in vitro transcription (IVT) using the MEGAshortscript ${ }^{\mathrm{TM}}$ T7 Transcription Kit (Thermo Fisher Scientific, Waltham, MA, USA) followed by T7-gRNA purification using the MEGAclear ${ }^{\mathrm{TM}}$ Transcription Clean-Up Kit (Thermo Fisher Scientific, Waltham, MA, USA) and elution in RNase-free ultrapure water.

DNA templates for in vitro cleavage were produced via PCR amplification using the primers listed in Table 8 and purified using the MinElute Kit (Qiagen, Hilden, Germany). Assembly of the digestion mixture was done by mixing $20 \mu \mathrm{l}$ water, $3 \mu \mathrm{l}$ of 10x Cas9 Nuclease Reaction Buffer (NEB, Ipswich, MA, USA), $3 \mu \mathrm{lgRNA}(300 \mathrm{nM})$ and $1 \mu \mathrm{l}$ S. pyogenes Cas9 (1 $\mu \mathrm{M})$ nuclease (NEB, Ipswich, MA, USA). After a pre-incubation at $25^{\circ} \mathrm{C}$ for $10 \mathrm{~min}, 3 \mu \mathrm{l}$ of DNA substrate (300 ng) were added and digestion was carried out at $37^{\circ} \mathrm{C}$ overnight. Fragment analysis was done using a 10\% Mini-PROTEAN ${ }^{\circledR}$ TBE Precast Gel (Bio-Rad, Munich, Germany).

\subsubsection{T7 Endonuclease I assay}

Human cells were transfected with $10 \mu \mathrm{g}$ of the respective guide RNA using electroporation. Genomic DNA was isolated 3-4 days later and used as a template for PCR amplification with primers listed in Table 9. PCR products were purified using a MinElute Kit (Qiagen, Hilden, Germany) and diluted with water to a final concentration of $20 \mathrm{ng} / \mu \mathrm{l} .10 \mu \mathrm{l}$ of PCR product were mixed with $2 \mu \mathrm{l}$ of 10x NEBuffer 2.1 (NEB, Ipswich, MA, USA) and $9 \mu$ water. For DNA hybridization the mixture was heated to $95^{\circ} \mathrm{C}$ for $5 \mathrm{~min}$ followed by cooling to $22^{\circ} \mathrm{C}$ with a cooling rate of $1.8^{\circ} \mathrm{C} / \mathrm{min}$ in a thermoyclcer. $1 \mu \mathrm{l}$ of T7 Endonuclease I (NEB, Ipswich, MA, 
USA) was added for $1 \mathrm{~h}$ at $37^{\circ} \mathrm{C}$ and a total DNA amount of $100 \mathrm{ng}$ loaded per lane using a 10\% Mini-PROTEAN ${ }^{\circledR}$ TBE Precast Gel (Bio-Rad, Munich, Germany).

\subsubsection{Genotyping of monoclonal cell lines}

Genomic DNA from monoclonal U2OS cells was isolated and used as a template for subsequent PCRs. Primers out-out PCRs and junction PCRs are listed in Table 10. Primers for off-target sites are listed in Table 11.

For DNA sequencing, PCR products were purified and ligated into a pCR ${ }^{\mathrm{TM}}$ Blunt II-TOPO ${ }^{\circledR}$ vector using a Zero Blunt ${ }^{\circledR}$ TOPO $^{\circledR}$ Kit (Thermo Fisher Scientific, Waltham, MA, USA) according to the manufacturer's instructions. Plasmids containing an insert were identified via colony PCR and 15 to 20 plasmids were sequenced per locus.

\subsection{Biochemical methods}

\subsubsection{Protein expression}

N-terminally 6xHis-tagged Dreiklang (DK) was prepared from E. coli cells carrying the pQE31DK expression plasmid. A preculture was prepared by inoculation of $250 \mathrm{ml}$ LB/Amp with one bacterial colony and grown at $37^{\circ} \mathrm{C}$ overnight while shaking. $10 \mathrm{ml}$ of a preculture were used for inoculation of $1 \mathrm{~L}$ main culture. Cells were grown at $25^{\circ} \mathrm{C}$ until an OD600 of about 0.4-0.6 was reached. Protein expression was induced by adding $1 \mathrm{mM}$ IPTG (isopropyl $\beta$-D-1thiogalactopyranoside) and the culture kept at $25^{\circ} \mathrm{C}$ overnight. Cells were harvested in a SLA1500 rotor at $5000 \mathrm{rpm}$ and $4{ }^{\circ} \mathrm{C}$ for $30 \mathrm{~min}$. Per gram of cell pellet $2 \mathrm{ml}$ resuspension buffer (10 mM Imidazole, $50 \mathrm{mM} \mathrm{NaH}_{2} \mathrm{PO}_{4}, 300 \mathrm{mM} \mathrm{NaCl}, \mathrm{pH}$ 8.0) containing lysozyme (1 $\mathrm{mg} / \mathrm{ml}$ ) and EDTA-free 1x Complete Protease Inhibitor (1 tablet per $50 \mathrm{ml}$ ) (RocheDiagnostics, Mannheim, Germany) was added. After resuspension the cells were incubated on ice for $2 \mathrm{~h}$ followed by sonication on ice (level 5, $60 \%$ pulse, $30 \mathrm{x}$ ) with a Branson Sonifier 450 (Emerson, St Louis, MO). The suspension was cleared by centrifugation at $4{ }^{\circ} \mathrm{C}$ and $15000 \mathrm{rpm}$ in a SS34 rotor for $2 \mathrm{~h}$. The supernatant containing $6 x$ His-tagged DK was used for the following protein purification steps.

\subsubsection{Protein purification}

For Ni2+-affinity chromatography a Vantage VL16 x 250 column (EMD Millipore, Billerica, MA, USA) filled with Ni-NTA Agarose (Qiagen, Hilden, Germany) was equilibrated with 3 column volumes of resuspension buffer. Protein extract containing 6xHis-Dreiklang was passed over 
the equilibrated column with a flow rate of $1 \mathrm{ml} / \mathrm{min}$ using a peristaltic pump. An ÄKTAprime plus purification system (GE Healthcare, Little Chalfort, UK) was used for washing steps and protein elution. Sequential washing was done with $50 \mathrm{ml}$ resuspension buffer containing 30, 50 and $70 \mathrm{mM}$ imidazole, respectively. Protein elution was done with resuspension buffer supplemented with $250 \mathrm{mM}$ imidazole. The flow rate during the purification procedure was 1 $\mathrm{ml} / \mathrm{min}$ with $10 \mathrm{ml}$ fractions $10 \mathrm{ml}$ during washing steps and $3 \mathrm{ml}$ fractions during the elution step. Concentration of pooled elution peak fractions was done using Vivaspin ${ }^{\circledR} 20$ centrifugal concentrators to a final volume of $2 \mathrm{ml}$ (Sartorius, Göttingen, Germany).

For size exclusion chromatography a HiLoad26/60 Superdex 200 (Pharmacia, Uppsala, Sweden) column was equilibrated with 3 column volumes of running buffer (100 mM Tris pH 7.5, $150 \mathrm{mM} \mathrm{NaCl}$ ). A flow rate of $0.3 \mathrm{ml} / \mathrm{min}$ was set at the ÄKTAprime plus purification system. The previously prepared $2 \mathrm{ml}$ concentrated protein solution was loaded and elution fractions of $0.2 \mathrm{ml}$ were taken. Peak elution fractions were pooled.

\subsubsection{Cell extract preparation for immunoblotting}

Cell extracts of subconfluent U2OS cells grown in a $10 \mathrm{~cm}$ dish were prepared by washing the cells two times in ice cold phosphate-buffered saline (PBS). The cells were scraped from the growth surface and resuspended in $500 \mu$ ice cold radioimmunoprecipitation assay (RIPA) buffer supplemented with $1 \mathrm{mM}$ EDTA, $1 \mathrm{mM}$ PMSF, $10 \mathrm{U} / \mathrm{ml}$ universal nuclease (Thermo Fisher Scientific, Waltham, MA, USA) and 1x complete protease inhibitor cocktail (Roche, Basel, Switzerland). For quantitative immunoblotting, the cells were detached from the growth surface using 0.05\% Trypsin/ 0.02\% EDTA (Biochrom, Berlin, Germany) and counted using a Scepter ${ }^{\mathrm{TM}} 2.0$ Cell counter (EMD Millipore, Billerica, MA, USA). Cells were harvested by centrifugation at 300xg for 2 min followed by lysis with $100 \mu$ RIPA buffer (supplemented as above) per $10^{6}$ cells.

After adding RIPA buffer, the cell suspension was placed on ice for 30 min with vortexing steps every $10 \mathrm{~min}$. The suspension was centrifuged at $13000 \mathrm{rpm}$ at $4^{\circ} \mathrm{C}$ for $30 \mathrm{~min}$. The supernatant was removed and the protein concentration measured using the Pierce BCA protein assay kit (Thermo Fisher Scientific, Waltham, MA, USA). Samples were diluted to 1.2 $\mu \mathrm{g} / \mu \mathrm{l}$ with RIPA buffer and mixed with the respective amount of $6 \mathrm{x}$ Laemmli buffer (375 mM Tris $\mathrm{pH} 6.8,12 \%$ SDS, $60 \%$ glycerol, $0.6 \mathrm{M} \mathrm{DTT}, 0.06 \%$ bromophenol blue) to a final concentration of $1 \mu \mathrm{g} / \mu \mathrm{l}$. The suspension was boiled at $95^{\circ} \mathrm{C}$ for $5 \mathrm{~min}$, flash frozen in liquid nitrogen and stored at $-20^{\circ} \mathrm{C}$ for further use. 


\subsubsection{SDS-PAGE}

For separation of protein mixtures, SDS-PAGE (sodium dodecyl sulfate polyacrylamide gel electrophoresis) was performed using the Laemmli buffer system (Laemmli, 1970). The acrylamide content of the separation gel ranged from 10-15\% and was adjusted to the molecular weight of the proteins to be separated. Per $10 \mathrm{ml}$ separating gel the following reagents were mixed:

\begin{tabular}{lccc}
\hline & \multicolumn{3}{c}{ Acrylamide perecentage } \\
Reagent & $\mathbf{1 0 \%}$ & $\mathbf{1 2 . 5 \%}$ & $\mathbf{1 5 \%}$ \\
\hline $\mathrm{H} 2 \mathrm{O}$ & $3.8 \mathrm{ml}$ & $3.2 \mathrm{ml}$ & $2.2 \mathrm{ml}$ \\
$30 \%$ Acrylamide $(\mathrm{w} / \mathrm{v}) /$ & & & \\
$0.8 \%$ Bisacrylamide $(\mathrm{w} / \mathrm{v})$ & $3.4 \mathrm{ml}$ & $4 \mathrm{ml}$ & $5 \mathrm{ml}$ \\
$1.5 \mathrm{M}$ Tris pH 8.8 & $2.6 \mathrm{ml}$ & $2.6 \mathrm{ml}$ & $2.6 \mathrm{ml}$ \\
$10 \%$ SDS $(\mathrm{w} / \mathrm{v})$ & $0.1 \mathrm{ml}$ & $0.1 \mathrm{ml}$ & $0.1 \mathrm{ml}$ \\
$10 \%$ APS $(\mathrm{w} / \mathrm{v})$ & $100 \mu \mathrm{l}$ & $100 \mu \mathrm{l}$ & $100 \mu \mathrm{l}$ \\
TEMED & $10 \mu \mathrm{l}$ & $10 \mu \mathrm{l}$ & $10 \mu \mathrm{l}$ \\
\hline
\end{tabular}

The acrylamide concentration of the stacking gel was $5 \%$. Therefore $5.6 \mathrm{ml} \mathrm{H} 2 \mathrm{O}, 2.5 \mathrm{ml} 1.5$ M Tris- $\mathrm{HCl} \mathrm{pH}$ 6.8, $1.7 \mathrm{ml}$ 30\% (w/v) Acrylamide/ 0.8\% (w/v) Bisacrylamide, $100 \mu \mathrm{l}$ 10\% SDS (w/v), $100 \mu \mathrm{l}$ 10\% (w/v) APS and $10 \mu \mathrm{l}$ TEMED were mixed. For quantitative western blotting, 4-15\% Mini-Protean ${ }^{\circledR}$ TGX $^{\text {TM }}$ Precast Gels (Bio-Rad, Munich, Germany) were used.

The gels were placed in a Mini-Protean ${ }^{\circledR}$ Tetra Cell (Bio-Rad, Munich, Germany) filled with $1 x$ SDS running buffer (25 mM Tris- $\mathrm{HCl}, 192 \mathrm{mM}$ glycerine, $0.1 \%$ (w/v) SDS, pH 8.3). Samples were mixed with 6x Laemmli buffer 6x Laemmli buffer (375 mM Tris pH 6.8, 12\% (w/v) SDS, $60 \%$ (v/v) glycerol, $0.6 \mathrm{M} \mathrm{DTT}, 0.06 \%$ (w/v) bromophenol blue) and boiled at $95^{\circ} \mathrm{C}$ for $5 \mathrm{~min}$. Samples were loaded on the gel together with a PageRuler Prestained Protein Ladder (MBI Fermentas, Burlington, Canada) as molecular weight marker. For electrophoretic protein separation a current of 25-50 mA per gel was applied until the bromophenol blue marker reached the bottom of the gel. Gels were stained in Coomassie Brilliant Blue staining solution (10\% (v/v) acetic acid, $25 \%(\mathrm{v} / \mathrm{v})$ isopropanol, $0.1 \%(\mathrm{w} / \mathrm{v})$ Coomassie Blue R-250) for 30 min and subsequently destained in $10 \%(\mathrm{v} / \mathrm{v})$ acetic acid solution.

\subsubsection{Western Blot}

Protein transfer after SDS-PAGE was done using a TE22 Mini Tank Transfer Unit (GE Healthcare, Freiburg, Germany). Proteins were transferred to a nitrocellulose membrane (GE 
Healthcare, Freiburg, Germany) in transfer buffer (25 mM Tris, $190 \mathrm{mM}$ glycine, 20\% methanol). Transfer was done at $4^{\circ} \mathrm{C}$ and $80 \mathrm{~mA}$ per membrane for $16 \mathrm{~h}$ with a magnetic stirrer circulating the transfer buffer. The membrane was briefly rinsed in TBS (50 mM Tris$\mathrm{HCl}, \mathrm{pH} 7.5,150 \mathrm{mM} \mathrm{NaCl})$ followed by incubation in Ponceau S solution (0.5 \% (w/v) Ponceau S, 40\% (v/v) MeOH, 15\% (v/v) acetic acid) for $10 \mathrm{~min}$ to visualize transferred proteins. The membrane was incubated in blocking buffer $(50 \mathrm{mM}$ Tris- $\mathrm{HCl}, \mathrm{pH}$ 7.5, $150 \mathrm{mM}$ $\mathrm{NaCl}, 0.1 \%$ Tween 20,5\% (w/v) skim milk) at room temperature for $1 \mathrm{~h}$. Primary antibodies were diluted in blocking buffer and incubated with the membrane at room temperature for $1 \mathrm{~h}$. The following primary antibodies were used: anti-HMGA1 (EPR7839; 1:5000; Abcam, Cambridge, UK), anti-Vimentin (V9; 1:1000; Santa Cruz Biotechnology, Heidelberg, Germany), anti-Zyxin (ZOL301, 1:1000, Abcam), anti-PHB1 (EP2803Y, 1:2000, Abcam), antiPHB2 (EPR14523, 1:5000, Abcam), anti-Actin (AC74; 1:3000, Sigma-Aldrich), anti-GFP (JL8; 1:3000, Clontech, Saint-Germain-en-Laye, France). After three washing steps with TBST (50 mM Tris- $\mathrm{HCl}, \mathrm{pH} 7.5,150 \mathrm{mM} \mathrm{NaCl}, 0.1 \%$ Tween 20), the membrane were incubated at room temperature with HRP-conjugated anti-rabbit or anti-mouse secondary antibodies (Dianova, Hamburg, Germany) diluted 1:5000 in blocking buffer for $1 \mathrm{~h}$. After three washing steps with TBST, the membrane was incubated with Pierce ECL western blotting substrate (Thermo Fisher Scientific, Waltham, MA, USA) and exposed to a CCD camera. Membranes were stripped using mild stripping buffer (15g Glycine, 0.001\% SDS, 0.01\% Tween 20, pH 2.2) or Restore ${ }^{\mathrm{TM}}$ Stripping Buffer (Thermo Fisher Scientific, Waltham, MA, USA) at $37^{\circ} \mathrm{C}$ for 30 min followed by applying the described protocol for reprobing with a different antibody.

\subsection{Light microscopy}

\subsubsection{Antibody coupling}

$2 \mathrm{mg}$ of the respective antibody were mixed with $100 \mu \mathrm{l} 1 \mathrm{M} \mathrm{NaHCO}_{3}$ and the respective fluorescent dye was dissolved in water free DMF to $10 \mu \mathrm{g} / \mu \mathrm{l} .20 \mu \mathrm{l}$ of dissolved dye were mixed with the antibody solution and incubated at RT for $1 \mathrm{~h}$. Subsequently $100 \mu \mathrm{l} \mathrm{M}$ Tris was added and the mixture stirred at RT for $5 \mathrm{~min}$. Purification of dye-coupled antibodies was done using a PD-10 desalting column (GE Healthcare, Little Chalfort, UK). Peak fractions were pooled, aliquoted to $50 \mu \mathrm{l}$ portions and flash frozen in liquid nitrogen for subsequent storage at $-80^{\circ} \mathrm{C}$. 


\subsubsection{Indirect immunofluorescence staining}

Human cells were cultured on glass cover slips until they reached a confluence of about $70 \%$ and fixed in $37^{\circ} \mathrm{C}$ prewarmed 4\% (w/v) PFA (paraformaldehyde) in PBS at RT for $5 \mathrm{~min}$. The cells were permeabilized using $0.5 \%(\mathrm{v} / \mathrm{v})$ Triton-X-100 in PBS for $5 \mathrm{~min}$ followed by subsequent incubation in blocking buffer (5\% (w/v) BSA in PBS containing $100 \mathrm{mM}$ glycin) for 5 min. Primary antibodies were diluted in blocking buffer and incubated with the coverslips at room temperature for $1 \mathrm{~h}$. The following primary antibodies were used: rabbit anti-HMG-I (EPR7839; 1:400; Abcam), mouse anti-Vimentin (V9; 1:100; Santa Cruz Biotechnology), mouse anti-Zyxin (ZOL301, 1:400, Abcam); rabbit anti-PHB1 (EP2803Y, 1:200, Abcam), rabbit anti-PHB2 (EPR14523, 1:400, Abcam); mouse anti-ESR1 (D12, 1:500, Santa Cruz Biotechnology), chicken anti-GFP (1:1000, Abcam). After three washing steps in PBS, fluorophore-coupled secondary antibodies were diluted 1:1000 and added for incubation at room temperature for $1 \mathrm{~h}$. The following secondary antibodies were used: sheep anti-mouse, goat anti-rabbit or goat anti-chicken (all Dianova, Hamburg, Germany) coupled to KK114 (Kolmakov et al., 2010) or Atto 590 (Atto-Tec, Siegen, Germany). After three PBS washing steps, cells were embedded in Mowiol 4-88 mounting medium containing $1 \mu \mathrm{g} / \mathrm{ml} \mathrm{4',6-}$ Diamidin-2-phenylindol (DAPI) and $2.5 \%$ (w/v) 1,4-diazabicyclo-[2,2,2]-octane (DABCO).

\subsubsection{Widefield microscopy}

Widefield fluorescence microscopy was done using an upright Leica DM6000 B epifluorescence microscope (Leica, Wetzlar, Germany). The microscope was equipped with a 100x oil immersion objective (HCX PL APO 100x/1.40-0.70 oil), a charge-coupled device (CCD) camera (DFC350FX) and various filter cubes: A4 (excitation: 360/40 nm; emission: 470/40 nm), L5 (excitation: 480/40 nm; emission: 527/30 nm), GFP (excitation: 470/40 nm; emission: 525/50 nm), N3 (excitation: 546/12 nm; mission: 600/40 nm), BGR (excitation: $420 / 30 \mathrm{~nm}, 495 / 15 \mathrm{~nm}$ or $570 / 20 \mathrm{~nm}$; emission: $465 / 20 \mathrm{~nm}, 530 / 30 \mathrm{~nm}$ or $640 / 40 \mathrm{~nm}$ ) and SFR (excitation: 630/20 nm; emission: 667/30 nm). Light source was a metal-halide lamp (EL6000, Leica Microsystems).

\subsubsection{Confocal microscopy}

Confocal microscopy was done using the Leica TCS SP5 Confocal Microscope (Leica, Wetzlar, Germany). All recordings were done using a pinhole diameter of one Airy unit (1.22N/NA), a scan speed of $400 \mathrm{~Hz}$ and a $63 \mathrm{x}$ oil immersion objective (HCX PL APO CS $63 \times / 1.40-0.60$ oil). The following laser lines were used for fluorescence excitation: a 405 
Diode (405 nm), an argon laser (458 nm/ 476 nm/ 488 nm/ 496 nm/ 514 nm), a diode-pumped solid-state (DPSS) laser (561 nm) and a helium-neon (HeNe) laser (630 nm). Fluorescence detection was done using photomultipliers (PMTs) operated within the dynamic range. Separation of excitation and emission light was accomplished using an AOTF (acousto-optic tunable filter). Multicolor imaging was done using sequential acquisition between frames. For image digitization a sampling rate according to the Nyquist criterion was chosen. Each image was recorded at least twice for averaging.

\subsubsection{STED super-resolution microscopy}

STED (stimulated emission depletion) super-resolution microscopy was done using an Abberior STED 775 QUAD scan nanoscope (Abberior Instruments, Göttingen, Germany). The nanoscope was equipped with a 100x oil immersion objective (Olympus UPlanSApo 100x/1.4). Fluorescence excitation was done using two pulsed laser sources at $594 \mathrm{~nm} 594$ nm (Abberior Instruments, Göttingen, Germany) and 640 nm (Picoquant, Berlin, Germany). Fluorescence depletion was achieved using a donut shaped pulsed laser at $775 \mathrm{~nm}$ leading to a lateral resolution of about $30 \mathrm{~nm}$. Image acquisition was done in the sequential linescanning mode. Image acquisition and processing was performed using the software ImSpector (Andreas Schönle, MPIbpc, Göttingen). Besides smoothing with a Gaussian filter and contrast stretching, no image processing was performed.

\subsubsection{RESOLFT super-resolution microscopy}

The home-built RESOLFT microscope utilized three separate beam paths for generating coaligned focal spots: two at a wavelength of $491 \mathrm{~nm}$ for excitation and OFF-switching, and one at $405 \mathrm{~nm}$ for ON-switching. The two focal spots at $491 \mathrm{~nm}$ comprised: (i) a normally focused pulsed beam for reading out the fluorescence signal; (ii) a 'doughnut-shaped' focal intensity distribution with a central minimum ('zero') for OFF-switching at the focal periphery in the xyplane, obtained by passing a continuous wave beam through a vortex phase mask (463nm mask, vortex plate VPP-A, RPC Photonics, Rochester, NY). The two focal intensity spots were generated by two different lasers diodes: one for OFF-switching (50 mW, continuous wave, Calypso 50, Cobolt, Stockholm, Sweden) and the second (10mW, 80-100ps pulse width PicoQuant, Berlin, Germany) for fluorescence readout. The third focal spot, again with a regularly focused profile, was generated by a laser diode at $405 \mathrm{~nm}$ wavelength (30mW, BCL030-405-S, CrystaLaser, Reno, NV, USA) and used for the ON-switching of the fluorescent protein. An oil-immersion objective lens (HCX PC APO, 100×, 1.4NA, oil; Leica Microsystems, 
Wetzlar, Germany) was used to image the different cell lines. A piezo actuator ENV40/20 (Piezosystem Jena, Jena, Germany) was used to move the objective lens along the optical axis in a range of $120 \mu \mathrm{m}$. A separate piezo stage NV40 (Piezosystem Jena) was implemented to translate the sample with nanometer precision in the xy-plane. The fluorescence signal was filtered by a bandpass filter $(532 / 70 \mathrm{~nm})$ and detected by an epitaxial silicon single photon avalanche diode SPAD (MPD, Bolzano, Italy); fluorescence photons were counted only when the $491 \mathrm{~nm}$ pulse read-out beam was switched on. The individual laser beam paths were triggered either by an acousto-optic modulator MTS 130A3 (Pegasus Optik $\mathrm{GmbH}$, Wallenhorst, Germany) or by an acousto-optic tunable filter AOTF.nC/TN (Pegasus Optik $\mathrm{GmbH}$ ). The pulse sequence and duration were defined by a pulse generator Model 9514 (QUANTUM COMPOSERS, Bozeman, MT, USA) and triggered by a timecorrelated single photon counting module (Becker \& Hickl, Berlin, Germany) pixel by pixel.

Each image was recorded by applying a specific pulse scheme, pixel by pixel. For details on all shown images, see Table 12. All intensity values are referring to the light intensities in the focal plane. Image acquisition and processing was performed using the software ImSpector (Andreas Schönle, MPIbpc, Göttingen).

The RESOLFT super-resolution microscope was built and operated by Dr. Ilaria Testa (Department of NanoBiophotonics, MPI-BPC, Göttingen, Germany).

\subsection{Electron Microscopy}

\subsubsection{Plastic embedding}

Human cells were grown on Aclar ${ }^{\circledR}$ polymer cover slips until 80-85\% confluence. Cells were prefixed in $2.5 \%(\mathrm{w} / \mathrm{v})$ glutaraldehyde in $0.1 \mathrm{M}$ sodium cacodylate at RT for 15 min postfixed in the same buffer at $4^{\circ} \mathrm{C}$ for $15 \mathrm{~h}$. Cells were washed three times in $0.1 \mathrm{M}$ sodium cacodylate and incubated in $1 \%(\mathrm{w} / \mathrm{v}) \mathrm{OsO}_{4}$ in $0.1 \mathrm{M}$ sodium cacodylate for $3 \mathrm{~h}$. Cells were washed once in $0.1 \mathrm{M}$ sodium cacodylate and then twice in water. The cells were place in $0.1 \%(\mathrm{w} / \mathrm{v})$ uranyl acetate (in $\mathrm{H}_{2} \mathrm{O}$ ) for 30 min. Uranyl acetate was washed out by subjecting the cells to $30 \%$ ethanol three times for 5 min followed by dehydration through a 50\%, 70\% and 100\% ethanol series. Afterwards the cells were placed in 100\% propylene oxide for $5 \mathrm{~min}$ and then transferred to $50 \% / 50 \%$ propylene oxide/Epon for $1 \mathrm{~h}$ followed by placement to $100 \%$ Epon overnight. Samples were sectioned to $50 \mathrm{~nm}$ thickness with a Leica EM UC6 ultramicrotome (Leica EM UC6, Leica Microsystems, Wetzlar, Germany). Each section was transferred to 0.7 \% (w/v) Pioloform coated 200 mesh carbon grids. Samples were subjected to postcontrasting using 1\% (w/v) uranyl- and lead acetate. Electron microscopic recordings were done using a 
Philips CM 120 transmission electron microscope equipped with a TVIPS $2 \mathrm{k} \times 2 \mathrm{k}$ slow-scan CCD camera (Philips, Amsterdam, Netherlands).

Sample preparation and image recording was done by Dr. Dietmar Riedel (Electron Microscopy Unit, MPI-BPC, Göttingen, Germany).

\subsubsection{Immunogold labelling}

Human cells were grown to $80-85 \%$ confluence and fixed in $37^{\circ} \mathrm{C}$ prewarmed $4 \%$ (w/v) PFA (paraformaldehyde) in PBS at RT for 30 min. Further sample processing was done according to Tokuyasu (1973). Samples were sectioned into $80 \mathrm{~nm}$ thin slices and incubated with diluted primary antibodies for $20 \mathrm{~min}$. The following antibodies were used: anti-GFP (JL-8; 1:20, Clontech, Saint-Germain-en-Laye, France), anti-PHB1 (EP2803Y, 1:20, Abcam), antiPHB2 (EPR14523, 1:20, Abcam). Subsequently each sample was incubated with protein A coupled to $10 \mathrm{~nm}$ gold particles for $20 \mathrm{~min}$ followed by multiple washing steps and postcontrasting using uranyl acetate/methylcellulose on ice for $10 \mathrm{~min}$. Electron microscopic recordings were done using a Philips CM 120 transmission electron microscope equipped with a TVIPS $2 \mathrm{k}$ x 2k slow-scan CCD camera (Philips, Amsterdam, Netherlands).

Sample preparation and image recording was done by Dr. Dirk Wenzel (Electron Microscopy Unit, MPI-BPC, Göttingen, Germany). 


\section{Results}

\subsection{Genomic tagging using zinc finger nucleases (ZFNs)}

State of the art genome editing methods were based on transcription activator-like effector nucleases (TALENs) or zinc finger nucleases (ZFNs) at the time when this project was initiated (Carroll, 2011; Joung and Sander, 2013). Both TALENs and ZFNs were used for generation of human and mouse cells expressing a fluorescent protein (FP) fusion protein from an endogenous locus and those cells were used for confocal microscopy studies (Hockemeyer et al., 2011; Samsonov et al., 2013). However, the first goal of this project was to establish endogenous FP labelling for RESOLFT (reversible saturable optical fluorescence transitions) super-resolution microscopy. Compared to conventional confocal microscopy, RESOLFT relies on the use of reversibly switchable FPs (RSFPs). Therefore, it first had to be tested whether a) RSFPs could be used for endogenous tagging in human cells and b) if endogenous expression levels of RSFP fusion proteins would be sufficient for RESOLFT super-resolution imaging.

Popular samples for demonstrating an improved labeling strategy or a new fluorophore for live cell super-resolution imaging are filamentous structures that form thin fibers or tubules (Godin et al., 2014). Of those structures we chose to target the human $\beta$-actin gene (ACTB) as actin overexpression has a severe impact on cell physiology (Mounier et al., 1997; Peckham et al., 2001) and ACTB-targeting ZFNs were commercially available (Figure 12).

a) Human $1 \mathrm{~kb}$ ACTB locus

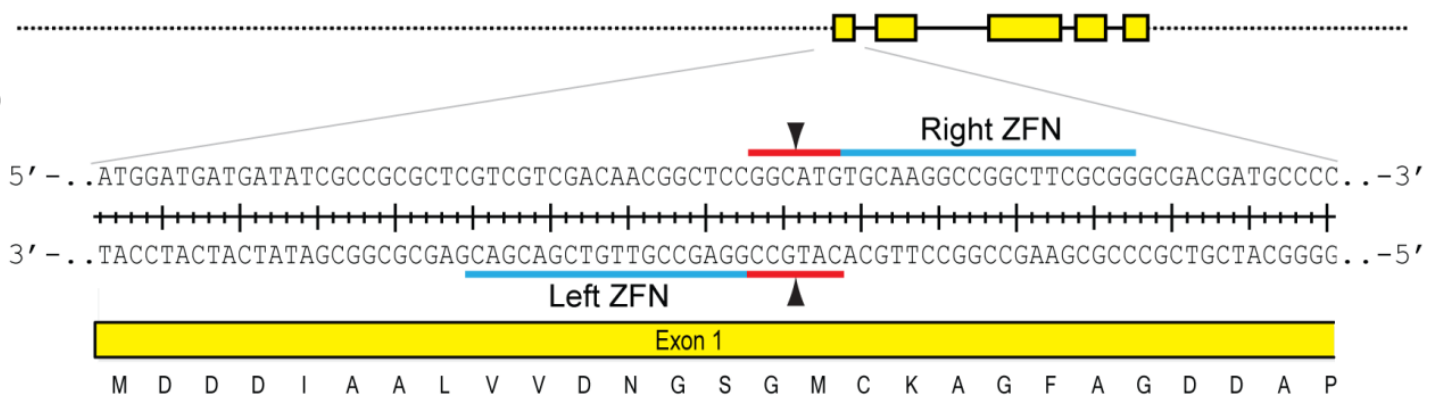

b)

Figure 12: Zinc finger nucleases targeting the human beta-actin gene. a) The gene structure of the human $\beta$-actin (ACTB) gene is shown. Yellow boxes: Exons; Black lines: introns; Dashed lines: Untranslated regions (UTRs). Note that the 5'-UTR comprises 37,127 bp of which only 5,000 bp are depicted. b) Zoom-in of start codon region. Zinc finger nucleases (ZFNs) bind to the respective target site (light blue), which leads to dimerization of Fokl nuclease domains (red) and induction of a double stranded break (DSB, arrows). 
Each ZFN carries a DNA-binding domain recognizing an 18 bp (left ZFN) or a 19 bp (right ZFN) nucleotide sequence. Further, each ZFN contains an obligate heterodimeric Fokl domain. This architectural feature inhibits undesired homodimerization events of ZFNs and leads to an improved on-target ZFN cleavage activity (Doyon et al., 2011). After DNA binding and Fokl heterodimerization, a DNA double strand break (DSB) is induced within the first exon of ACTB.

A DSB can be repaired using the cellular pathway of non-homologous end-joining (NHEJ) or homology-directed repair (HDR) (Jasin and Rothstein, 2013). Whereas NHEJ is an errorprone process potentially disrupting gene function, HDR can be used for site-specific transgene integration. By providing a donor plasmid containing homology arms that flank the transgene of interest, HDR was used for N-terminal tagging of ACTB (Figure 13).

a)

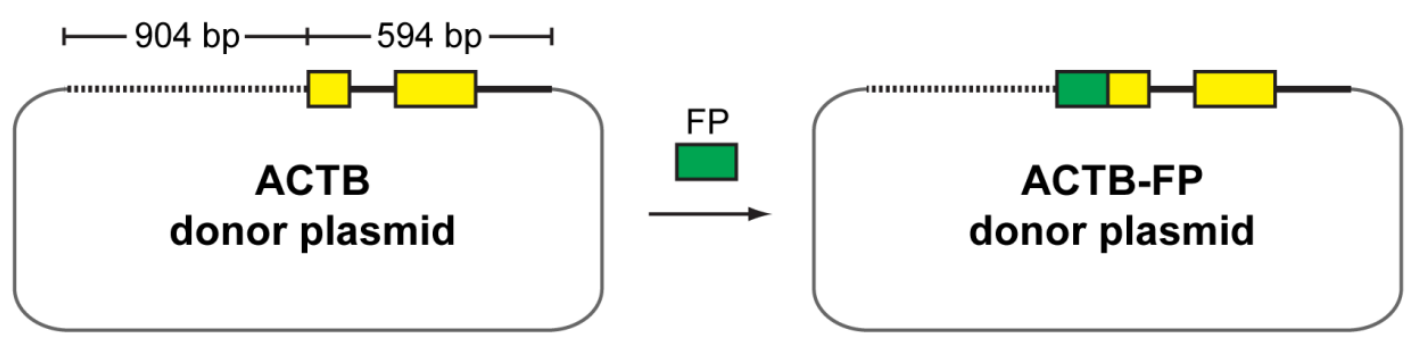

b)

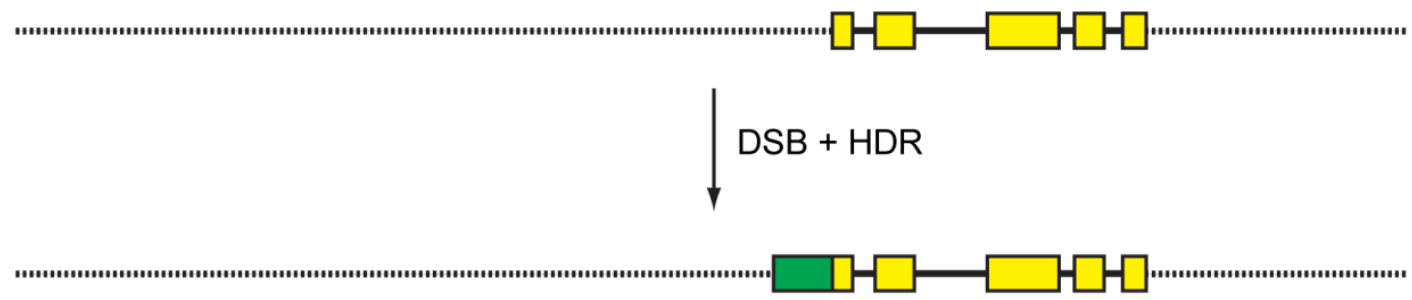

Figure 13: Donor plasmids for endogenous tagging of $\beta$-actin. a) An empty donor plasmid bearing a left homology arm of 904 bp and a right homology arm of 594 bp was obtained via gene synthesis. The DNA sequence for the fluorescent protein (FP) of interest (Dreiklang, rsEGFP or rsEGFP2, respectively) was inserted between the homology arms using molecular cloning. b) Zinc finger nucleases induce a site-specific double stranded break (DSB) at the human actin gene. Homologydirected repair (HDR) using the FP containing donor plasmid as repair template leads to site-specific FP integration upstream of the first exon.

Specifically, the donor plasmid was designed to contain a left homology arm (LHA) of 904 bp spanning part of the 5'-UTR of ACTB; the right homology arm (RHA) had a length of $594 \mathrm{bp}$ and included both exon 1 and 2 as well as intron 1 and 2 of ACTB. The RSFPs inserted between LHA and RHA were rsEGFP (Grotjohann et al., 2011), rsEGFP2 (Grotjohann et al., 
2012) and Dreiklang (Brakemann et al., 2011), respectively. Successful HDR-mediated RSFP integration upstream of exon 1 would lead to endogenously tagged $\beta$-actin leaving all other regions of the gene unmodified.

For genomic tagging, U2OS cells were co-transfected with the ACTB-targeting ZFN pair and the respective donor plasmid. Seven days after transfection, the cells were analyzed using confocal fluorescence microscopy. Inspection of DAPI-stained polyclonal cultures showed the presence of fluorescent $\beta$-actin for all tested RSFPS, indicating the successful RSFP integration at the ACTB locus (Figure 14). While F-actin could be detected as typical filamentous fibers, a high fluorescent background caused by diffusing soluble G-actin was present.

a)

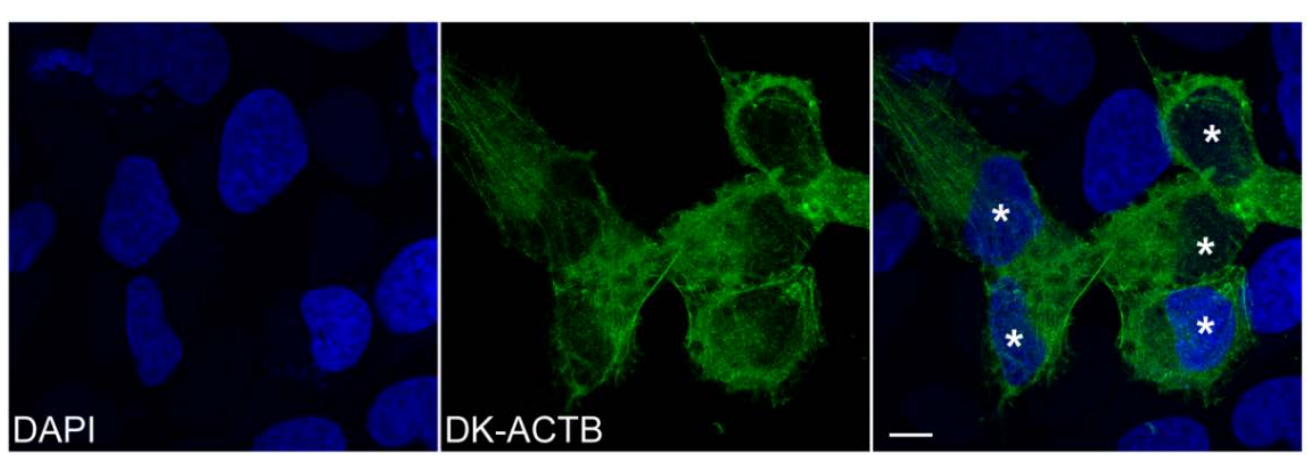

b)

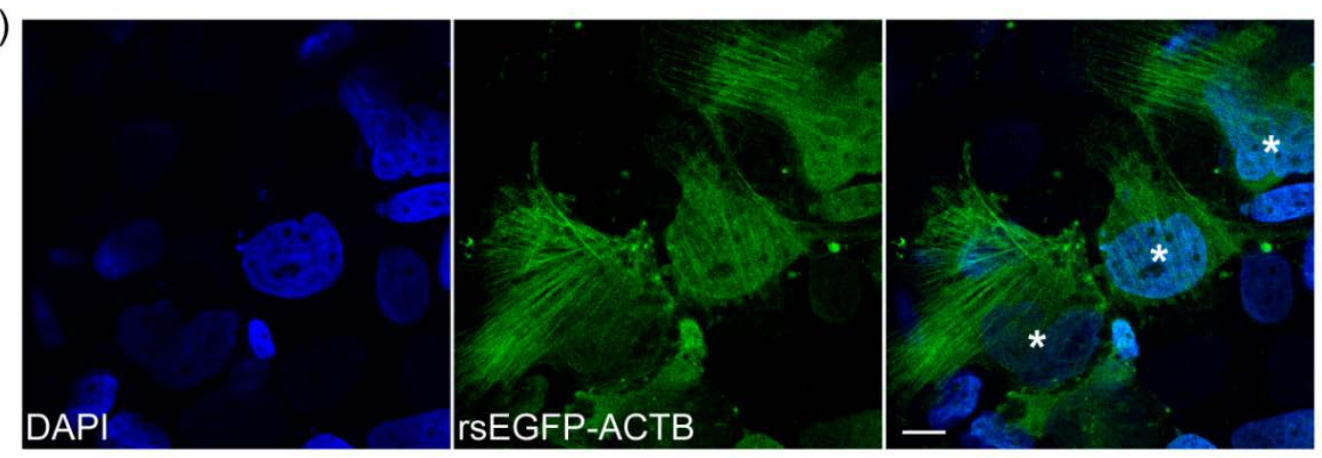

c)
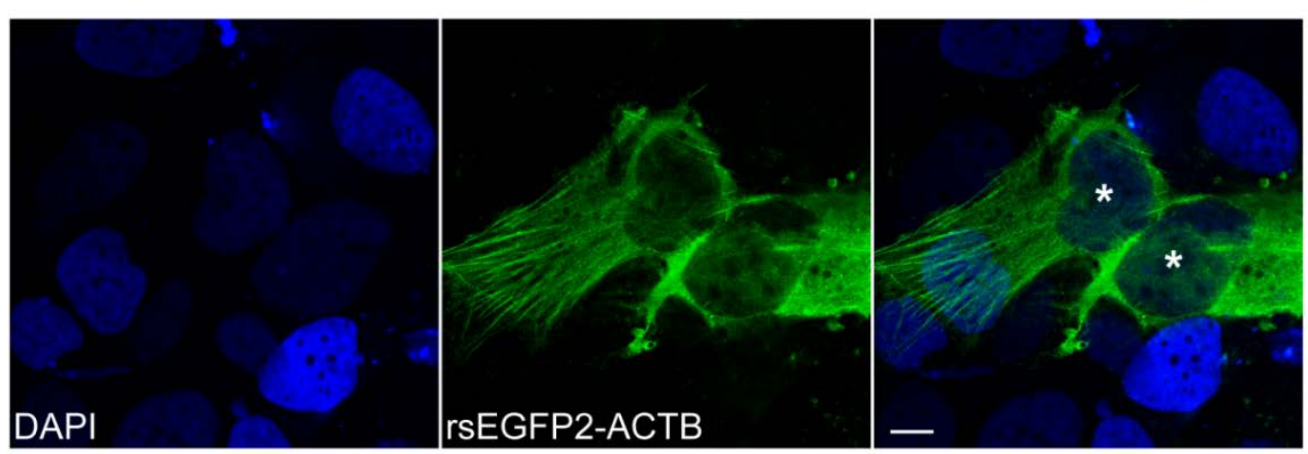

Figure 14: Endogenous tagging of $\beta$-actin with RSFPs. Confocal images of fixed U2OS cells taken seven days after co-transfection with both ZFN plasmids and the respective donor plasmid. Site- 
specific donor plasmid Integration leads to endogenous expression of Dreiklang- $\beta$-actin (a), rsEGFP- $\beta$ actin (b) and rsEGFP2- $\beta$-actin (c) in a fraction of cells (white asterisks). DAPI was used to visualize all cells including non-targeted ones. Scale bar: $10 \mu \mathrm{m}$.

As an initial proof of principle, the Dreiklang- $\beta$-actin (DK-ACTB) polyclonal culture was chosen for live cell RESOLFT imaging. Among the three RSFPs used, Dreiklang (DK) shows a unique switching mechanism and a high molecular brightness making it a beneficial probe for deterministic as well as stochastic super-resolution microscopy methods (Brakemann et al., 2011; Jensen et al., 2014). DK-ACTB cells were successfully imaged using RESOLFT, which showed an increase in lateral resolution (Figure 15). Unfortunately, DK exhibits slow switching kinetics leading to high pixel dwell times. Further, actin filaments are dynamic and the soluble DK-tagged form of actin, G-actin, caused high background fluorescence. Therefore, highly dynamic thin actin filaments could not be recorded and only thicker, more stable structures were visible at good signal to noise ratios.

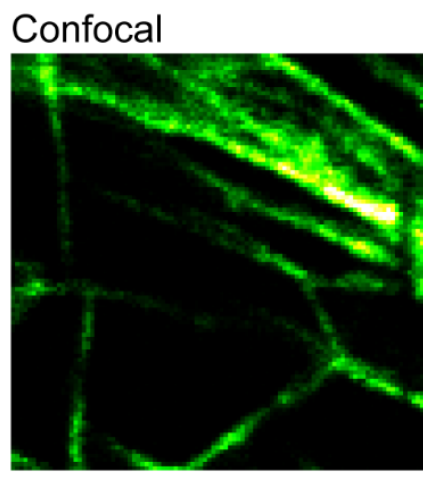

RESOLFT
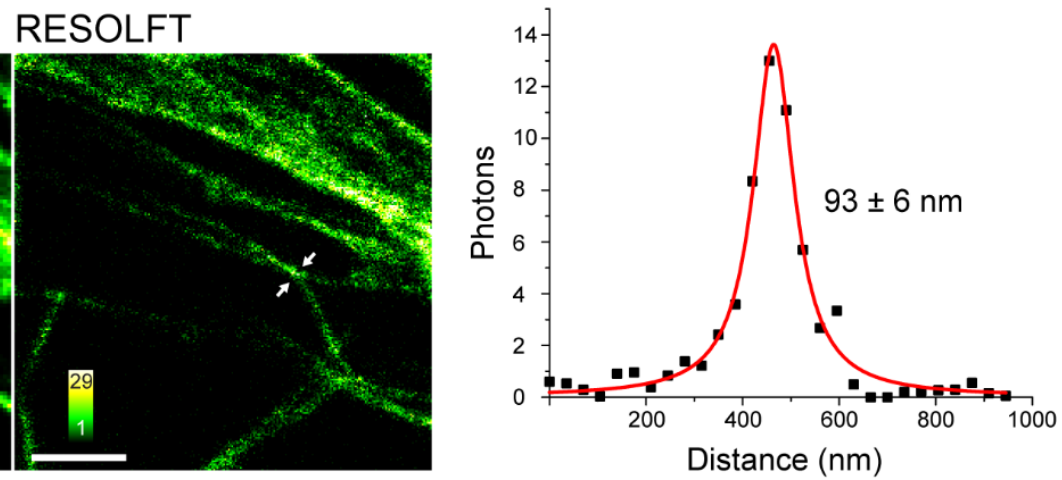

Figure 15: Live-cell RESOLFT nanoscopy on DK-actin cells. Compared to confocal mode, RESOLFT imaging results in an enhanced resolution. Due to the high dynamics of thin actin filaments, only thicker and more stable structures are visible at good signal to noise ratios. Scale bar: $2 \mu \mathrm{m}$.

Next, rsEGFP2- $\beta$-actin (rsEGFP2-ACTB) cells were chosen for RESOLFT imaging. Compared to DK, rsEGFP2 is more suitable for imaging dynamic structures in living cells due to its faster switching kinetics (Grotjohann et al., 2012). RESOLFT could successfully capture dynamic changes of F-actin fibers in rsEGFP2-ACTB cells (Figure 16). Structural rearrangements of the cortical actin network within living endogenously tagged human cells could be recorded without any observable photodamage. However, the achieved resolution was only slightly improved compared to confocal microscopy. This was due to low signal-tonoise ratio in regions with thin filaments caused by the presence of soluble fluorescent Gactin. In fact, imaging was mainly performed on thicker stress fibers that reach a diameter of up to $180 \mathrm{~nm}$ (Tojkander et al., 2012). 
In summary, it could be demonstrated that ZFN-mediated integration of RSFPs at the ACTB locus is possible, leading to endogenous expression of RSFP-tagged $\beta$-actin in human U2OS cells. While RESOLFT imaging was principally shown to work on DK-ACTB and rsEGFP2ACTB cells, the obtained resolution was not satisfactory, because simultaneous labeling of Gactin next to F-actin causes a high cellular background and F-actin stress fibers are very thick structures. Therefore, actin is not a suitable the technical sample to test genomic tagging as labeling strategy for live cell RESOLFT super-resolution imaging. However, the dependence on ZFNs imposed restrictions on target protein selection for endogenous tagging. While more ZFNs could be generated to target other proteins than actin, assembly and verification of ZFNs for other targets is an expensive and time-consuming process that requires expert knowledge in protein engineering (Gonzalez et al., 2010;Sander et al., 2011). Compared to ZFNs, generation and validation of TALENs is more straightforward, but their highly repetitive DNA sequences and large size render synthesis and subsequent delivery difficult and inefficient.

a)
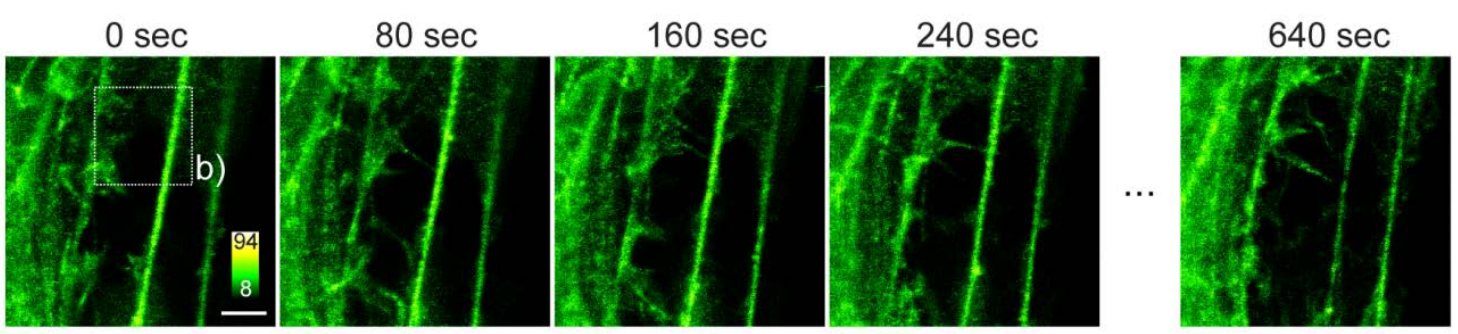

b)
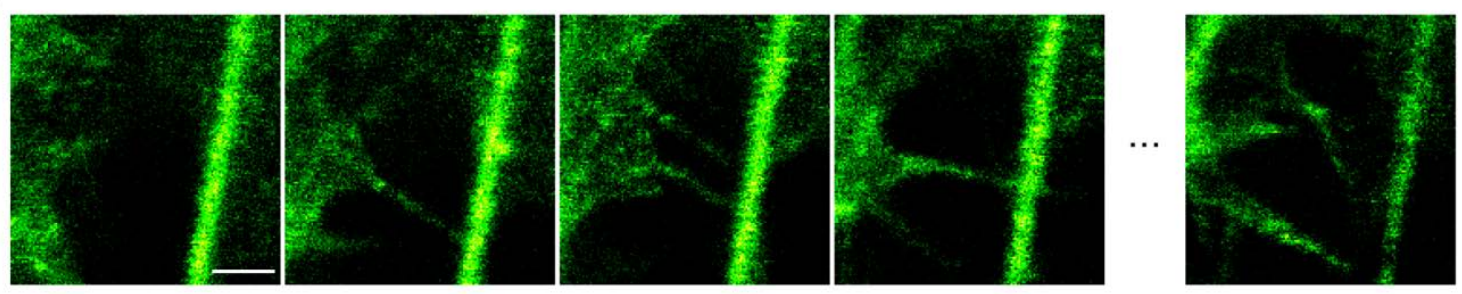

Figure 16: Live-cell RESOLFT nanoscopy on rsEGFP2-actin cells. a) Dynamic changes of the actin cytoskeleton were captured in a large field of view over several minutes. b) Zoom-in of the indicated region highlights dynamic changes of actin filaments over time. Scale bar: $2 \mu \mathrm{m}$.

Therefore, to explore the full potential of site-specific endogenous tagging for imaging and subsequent applications, a simple and more rapid genome editing system was required. Moreover, an entire workflow starting from nuclease design to generation and thorough characterization of monoclonal cell lines had to be established. Instead of relying on ZFNs or TALEN, we set out to change and use the more efficient CRISPR-Cas9 system for further genome editing studies (Jinek et al., 2012; Cong et al., 2013; Mali et al., 2013). 


\subsection{Establishment of a general workflow for genome editing of human cells using CRISPR}

Recently, the type II clustered regularly interspaced short palindromic repeats (CRISPR) system and the CRISPR-associated (Cas) endonuclease Cas9 were engineered into a powerful genome editing tool (Hsu et al., 2014; Sander and Joung, 2014). Cas9 can be targeted to induce a DNA double-strand break (DSB) at any genomic site defined by a 20 bp long guide RNA (gRNA) sequence complementary to the target site flanked by a NGG protospacer adjacent motif (PAM). Compared to genome editing based ZFNs or TALENs, CRISPR-Cas9 is an inexpensive and simple-to-use tool that allows for rapid site-specific genome modifications. Therefore, we decided to focus on the establishment of a robust protocol for the generation of human knock-in cells using the CRISPR-Cas9 system as outlined within the following section.

\subsubsection{Target selection and generation of gRNAs}

The DNA sequence of a respective human gene was retrieved from the NCBI Gene database (http://www.ncbi.nlm.nih.gov/gene) and a region of interest within the gene used for guide RNA design based on the CRISPR Design Tool (Hsu et al., 2013; http://crispr.mit.edu). This web-based tool identifies all S. pyogenes Cas9 target sites within an input DNA sequence and ranks potential gRNAs based on their on- and off-target activity. Further, it implements experimental data regarding gRNA quality (Hsu et al., 2013) and serves as a powerful tool to design highly specific and efficient gRNAs as demonstrated in later sections. Target site selection is demonstrated for C-terminal tagging of the human gene encoding for the cytoskeletal protein vimentin (Figure 17).

a)

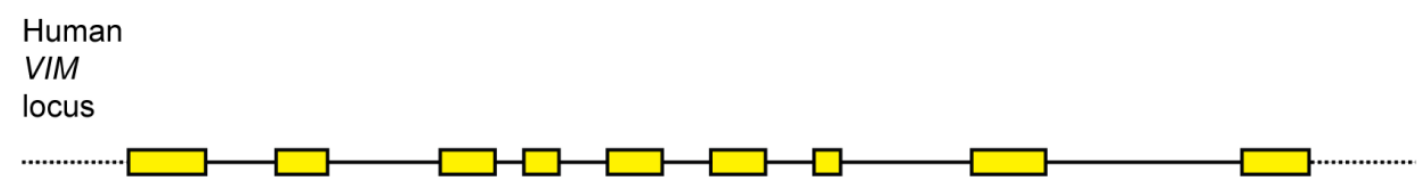

b)

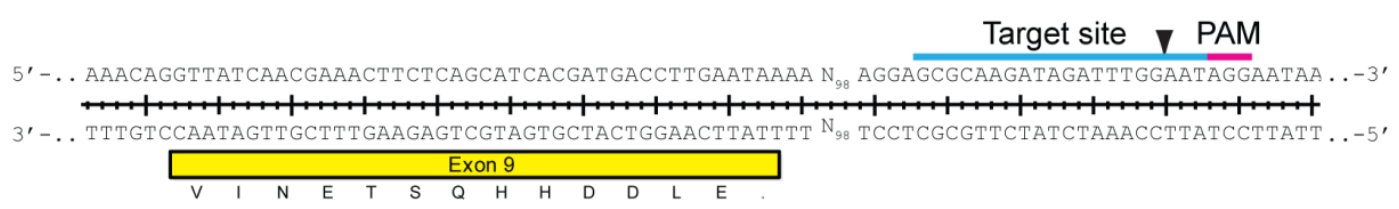

Figure 17: Schematic for target site selection within a human gene. a) The gene structure of the human vimentin (VIM) gene is shown. Yellow boxes: Exons; Solid lines: introns; Dashed lines: 5'- and 3'-UTR. b) Zoom-in of stop codon region. For C-terminal transgene integration, a DNA double-strand 
break (DSB) must be induced within the stop codon region of the terminal exon (Exon 9). S. pyogenes Cas9 targets a 20 bp sequence (light blue) which must be followed at its 3 '-end by the NGG protospacer adjacent motif (PAM, pink). Cas9 induces a DSB at a site 3 bp upstream of the PAM (black arrowhead).

Based on the selected gRNA, a pair of oligonucleotides was designed that contained a) the 20 bp DNA target site and b) overhangs compatible for cloning into a Bbsl-digested plasmid backbone (Figure 18). We chose to work with the bicistronic CRISPR backbone pX330 that contains two expression cassettes: human codon-optimized Cas9 and a chimeric gRNA (Cong et al., 2013). Other CRISPR expression systems for mammalian cells rely on the cotransfection of two separate plasmids encoding for Cas9 and gRNA (Mali et al., 2013; Jinek et al., 2013). As endogenous tagging requires the presence of an additional repair plasmid, we reasoned that plasmid co-delivery rates are higher when two instead of three plasmids are used in total. Moreover, pX330 contains a longer chimeric gRNA which was shown to increase targeting efficiency compared to previous CRISPR expression systems (Cong et al., 2013). It should be noted that $p \times 330$ uses a U6 RNA polymerase III promoter to drive gRNA expression and the U6 promoter prefers a guanine $(G)$ nucleotide as the first base of its transcript (Guschin et al., 2010). Therefore, an extra G must be added at the 5' end of the gRNA in case the 20 bp guide sequence does not begin with $G$.

a)

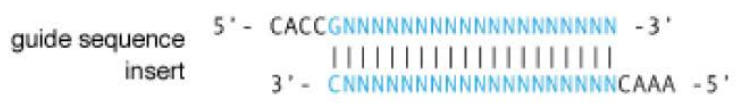

b)

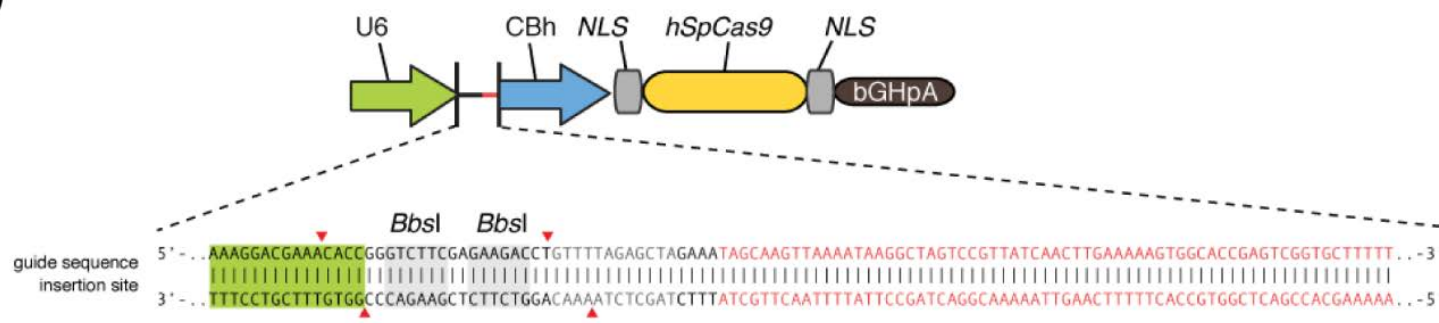

Figure 18: Schematic for oligo cloning into plasmid pX330. a) Two oligonucleotides are designed based on the $20 \mathrm{bp}$ target site (light blue letters). The guide oligos contain Bbsl compatible overhangs (black letters) for ligation into pX330. A G-C base pair is added at the $5^{\prime}$ end of the guide sequence for U6 promoter driven transcription, which does not adversely affect targeting efficiency. b) The plasmid pX330 contains two expression cassettes: human codon-optimized S. pyogenes Cas9 (hSpCas9) and the chimeric guide RNA. Digestion of pX330 with Bbsl allows the replacement of the Bbsl restriction sites with direct insertion of annealed oligos. (Adapted from Cong et al., 2013 with permission from AAAS). 
For cloning, the hybridized oligonucleotide pair was ligated downstream of the U6 promoter into Bbsl-digested pX330 followed by heat-shock of $E$. coli cells. Identification of positive bacterial clones was done via colony PCR using an insert-specific forward primer and a universal pX330-specific reverse primer (Figure 19). Plasmid modification with annealed oligonucleotides is a rapid and highly efficient one-step process which generally does not require to screen more than three bacterial colonies per gRNA. Moreover, the cloning step can be parallelized and multiple pX330-based expression plasmids can be generated and sequence-verified easily within 3 days (Ran et al., 2013b). This is in stark contrast to the generation of ZFNs or TALENs which is laborious and requires 5-7 days (Wright et al., 2006; Sanjana et al., 2012). Positive, gRNA-containing clones were used for plasmid DNA isolation, DNA sequencing and subsequent genome-editing experiments. An overview of the cloned gRNAs within this work is given in Table 13.

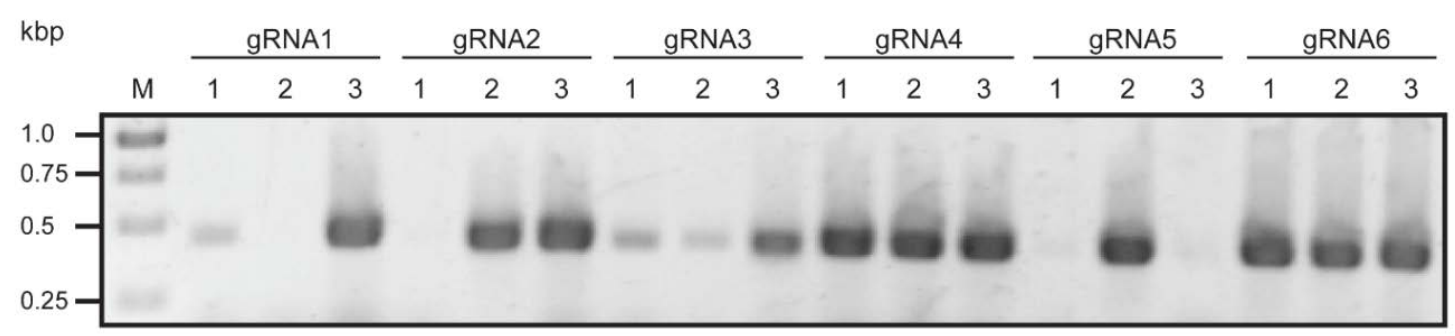

Figure 19: Colony PCR after oligo cloning in pX330. A typical example for determining the presence or absence of insert DNA after oligo cloning is shown for six different gRNAs. Three clones per gRNA were analyzed using an insert-specific forward primer and a universal reverse primer annealing within pX330. Constructs containing the oligo insert show a 442 bp band, whereas constructs without oligo insert show no band.

\subsubsection{Functional validation of $g R N A s$}

After target site selection and gRNA cloning it is required to determine whether the respective gRNA forms an active complex with Cas9 capable of inducing a site-specific DNA double strand break (DSB). Therefore, two assays were established: 1) an in vitro assay based on reconstituted gRNA/Cas9 complex and 2) an in vivo assay based on DNA mismatch-specific endonuclease I T7 (T7EI).

For in vitro activity testing of gRNA/Cas9 pairs, the respective gRNA had to be transcribed. Therefore, a PCR cassette containing a minimal T7 promoter and the gRNA sequence was amplified from the respective gRNA-containing plasmid. The amplicon was used as a template for in vitro gRNA transcription yielding a $101 \mathrm{bp}$ long gRNA. A DNA fragment containing the respective Cas9 cleavage site was amplified from wildtype U2OS genomic 
DNA. Cas9 and gRNA were mixed in a molar stoichiometry of 1:1. After a brief pre-incubation period, the substrate DNA was added for an overnight incubation step at $37^{\circ} \mathrm{C}$ followed by analysis via native polyacrylamide gel electrophoresis (Figure 20).

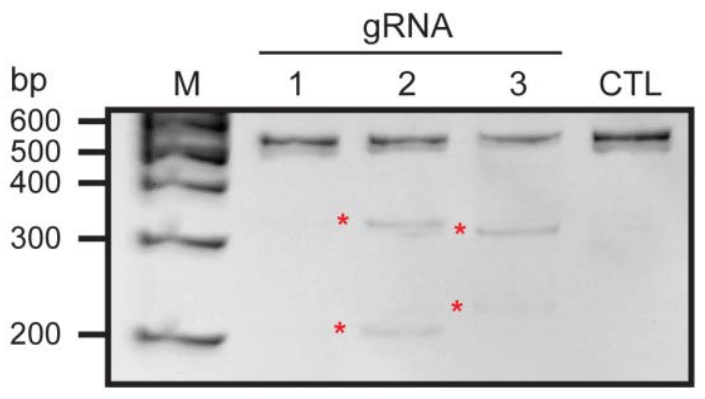

Figure 20: in vitro Cas9/gRNA cleavage assay. Efficacy of three different gRNAs targeting the same genomic region at various DNA sites was tested in vitro. DNA templates that contain Cas9/gRNA target sites were produced via PCR using U2OS genomic DNA as a template. Recombinant Cas9 and in vitro transcribed gRNAs were used for reconstitution of Cas9/gRNA complexes. Inactive Cas9/gRNA complexes do not produce any cleavage products as observed for Cas9-untreated control DNA templates (CTL). Active Cas9/gRNA complexes cleave DNA templates into fragments of characteristic size (red asterisks) dependent on the nuclease cleavage site.

The in vitro assay results suggest that Cas9 together with gRNA1 does not result in endonucleolytic cleavage of the DNA substrate, while both gRNA2 and gRNA3 lead to Cas9mediated DNA cleavage in vitro. The advantage of validating gRNAs in vitro is that the assay is robust and takes only 1-2 days. However, some gRNA/Cas9 combinations exhibit in vitro activity, while local chromatin structures might block a locus of interest in vivo (Wu et al., 2014b; Kuscu et al., 2014). In order to take such factors into account, an alternative in vivo assay was established.

For in vivo activity testing of gRNA/Cas9 pairs, an assay involving the mismatch-specific nuclease T7EI was used. This assay is based on the ability of T7EI to recognize and cleave DNA sequence mismatches due to the presence of small insertions or deletions (indels) that were previously introduced by Cas9 (Mashal et al., 1995). U2OS cells were electroporated with the respective Cas9/gRNA-expressing plasmid to induce site-specific mutagenesis. Three days after transfection, genomic DNA was isolated and used as a template for PCR amplification. PCR primers were designed such that a 300-800 bp long amplicon was generated that contains the Cas9 cleavage site. After denaturation and reannealing of $200 \mathrm{ng}$ purified PCR products, T7El was added and the mixture incubated for $1 \mathrm{~h}$ at $37^{\circ} \mathrm{C}$. For DNA 
cleavage analysis, the mixture was separated using a 10\% native polyacrylamide gel (Figure 21).

a)

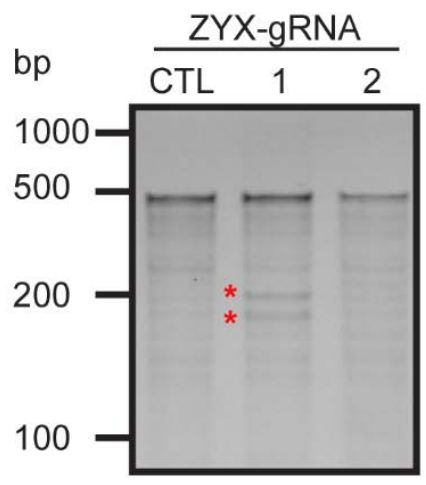

b)

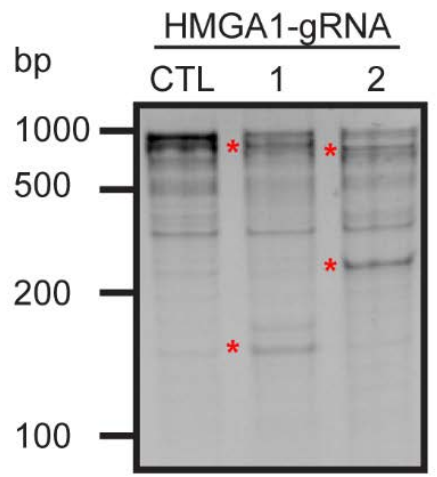

Figure 21: T7 Endonuclease I (T7EI) assay to test Cas9/gRNA activity in vivo. U2OS cells were transfected with two gRNAs that target the human zyxin (ZYX) locus (a) or the human high mobility group protein A1 (HMGA1) locus (b), respectively. Control cells (CTL) were transfected with empty pX330. Genomic DNA was isolated and used as template for T7El substrate preparation. ZYX-gRNA1, HMGA1-gRNA1 and HMGA1-gRNA2 successfully induce mutations at the targeted locus while ZYXgRNA2 does not modify the target site.

The observed cleavage pattern indicates that ZYX-gRNA1, HMGA1-gRNA1 and HMGA1gRNA2, introduced a DSB that resulted in indel mutations at the respective target site. The advantage of using the T7El assay over an in vitro assay is that gRNA functionality is validated in vivo and factors such as locus accessibility or DNA modifications are therefore considered. The disadvantage of T7EI is that it takes 3-4 days to complete and the sensitivity is about $5 \%$ due to high background cleavage occurring even on perfectly matched DNA (Vouillot et al., 2015).

Both assays are useful to evaluate the capability of a gRNA to induce a DSB in vitro or in vivo. This is important for knockout studies, where efficiently cutting Cas9/gRNA pairs should be used, because mutations are induced more frequently then and fewer clones must be screened before a knockout is obtained (Ran et al., 2013b). However, the main objective of this thesis was to use CRISPR for endogenous FP tagging that requires homologous recombination (HR) at a given locus. HR frequencies depend on the DNA methylation status, chromatin structure and the nature of the specific sequences that undergo HR (Liang and Jasin, 1995; Ramdas and Muniyappa, 1995). Thus, even an efficient Cas9/gRNA pair might not be useful for HR-dependent integration of a fluorescent reporter. Therefore, instead of testing multiple gRNAs for mutagenic activity, the Cas9/gRNA pairs were directly validated according to their ability to mediate FP integration at the locus of interest in presence of a 
donor repair template. The results of this approach are in full agreement with the obtained T7EI data as demonstrated later on.

\subsubsection{Generation of donor plasmids}

Next to a functional nuclease, the second component required for site-specific gene tagging with fluorescent reporters is a donor plasmid. The donor plasmid is based on a pUC57 backbone and contains two gene-specific homology arms with a length of 500-1000 bp each that flank the transgene of interest. Generation of donor plasmids is cumbersome and timeconsuming since it generally relies on three or more cloning steps (Wu et al., 2008). A more rapid generation of any donor plasmid is desired and therefore two cloning strategies serving this purpose were established within this work: 1) two-step four fragments cloning and 2) onestep Gibson assembly (Gibson et al., 2009).

In general, both cloning strategies rely on the use of pUC57 as a vector backbone, a left homology arm (LHA) and a right homology arm (RHA) DNA sequence amplified from the genomic region of interest; the fluorescent protein DNA is amplified from a plasmid template. For four fragments cloning, each amplicon must contain a unique restriction site at its 5'- and 3'-end, respectively, compatible with the neighboring DNA fragment. After amplicon purification, the three amplicons and the backbone are digested separately using restriction enzymes followed by DNA ligation (Figure 22). The ligation reaction is used for electroporation of $E$. coli cells and positive bacterial clones can be identified subsequently using colony PCR.

a)

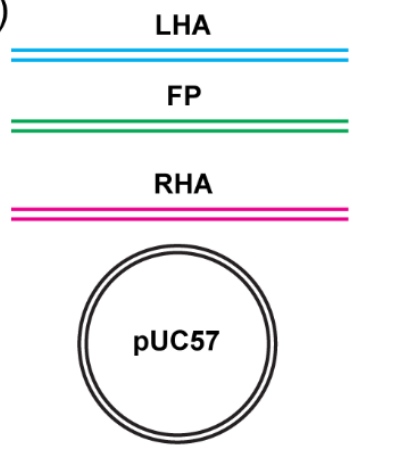

b)

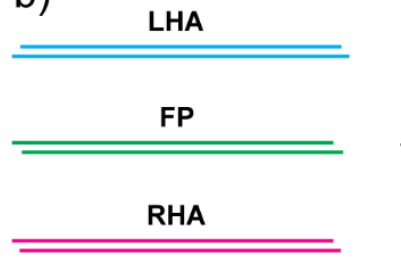$$
\text { ) }
$$

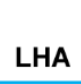

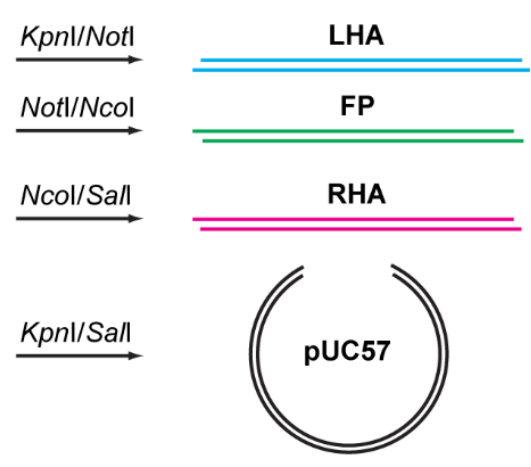

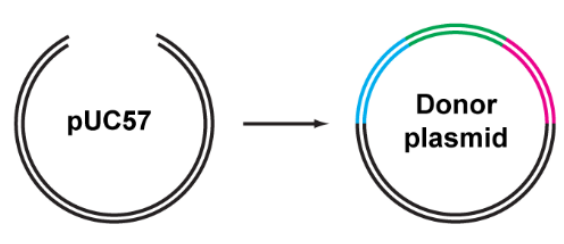

Figure 22: Two-step four fragment cloning strategy for donor plasmid construction. a) Three inserts are prepared via PCR. Double digest of each insert and the pUC57 vector backbone creates 
restriction enzyme specific 5' or 3' overhangs that facilitate annealing of complementary fragments. b) Ligation of complementary fragments and backbone yields the final donor plasmid.

For Gibson assembly (Gibson et al., 2009) each amplicon must contain unique 5'- and 3'overhangs compatible with the neighboring DNA fragment. After amplicon purification, the amplicons and the linearized pUC57 backbone are digested and ligated in one step (Figure 23). The ligation reaction is then used for $E$. coli transformation via heat-shock and subsequent colony PCR analysis for identification of bacterial clones containing the correct plasmid.

In conclusion, both cloning strategies can be applied to construct a donor plasmid within 4 days. However, the preferred method for donor plasmid generation is Gibson assembly, because it does not rely on the use of restriction enzymes and therefore allows greater flexibility in plasmid design. Further, no restriction site remains between neighboring DNA fragments, which is crucial when two adjacent fragments reconstitute a coding exon. All donor plasmids generated over the course of this thesis are given in Table 14.

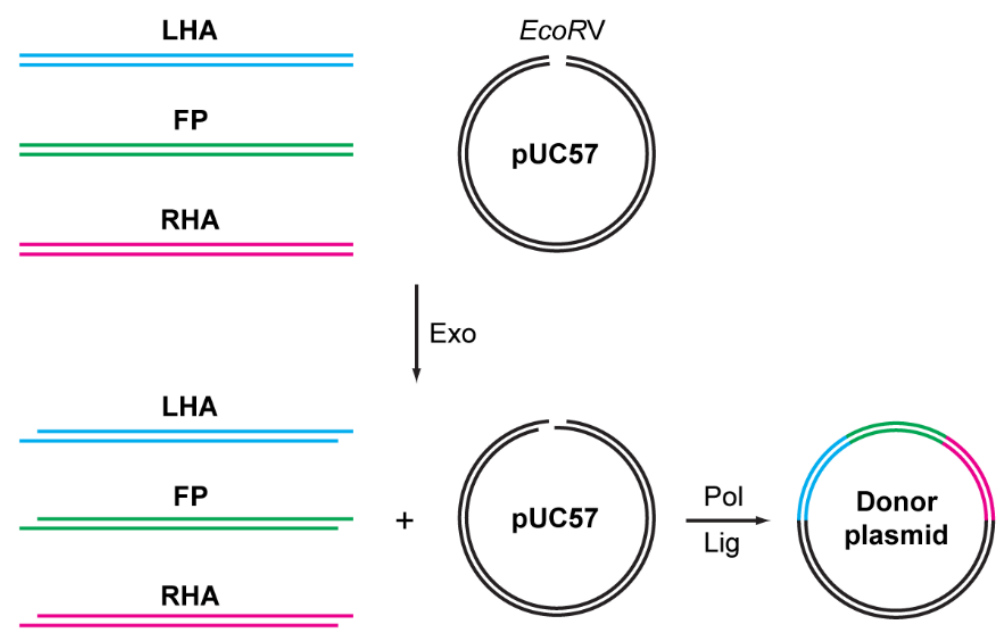

Figure 23: One-step Gibson assembly for donor plasmid construction. Three inserts are prepared via PCR and mixed with EcoRV-linearized pUC57 plasmid. All four fragments are joined in a single tube isothermal reaction. T5 exonuclease (Exo) creates single-stranded 3' overhangs that facilitate annealing of complementary fragments. Q5 DNA polymerase (Pol) fills in gaps within each annealed fragment. T4 ligase (Lig) seals nicks in the assembled DNA to yield the final donor plasmid.

\subsubsection{Clone isolation and clonal expansion}

After generation of gRNA and donor plasmids required for site-specific gene tagging, U2OS cells were co-transfected with both constructs. Since successfully targeted cells would express a fluorescent reporter, no further antibiotic selection markers were used. To obtain monoclonal cell lines, single cells were isolated using fluorescent activated cell sorting 
(FACS) seven to ten days after transfection (Figure 24). Clonal expansion was done in 96well plates for about two weeks. Wells containing cell colonies were detected using a brightfield microscope. Those cells were detached from the growth surface using trypsin and transferred into a 24-well plate. After cultivation for another three to five days, the cells were split and replicated on a second 24-well plate containing glass cover slips for fluorescence microscopy analysis. Positively targeted fluorescent cells were kept in culture for further experiments.
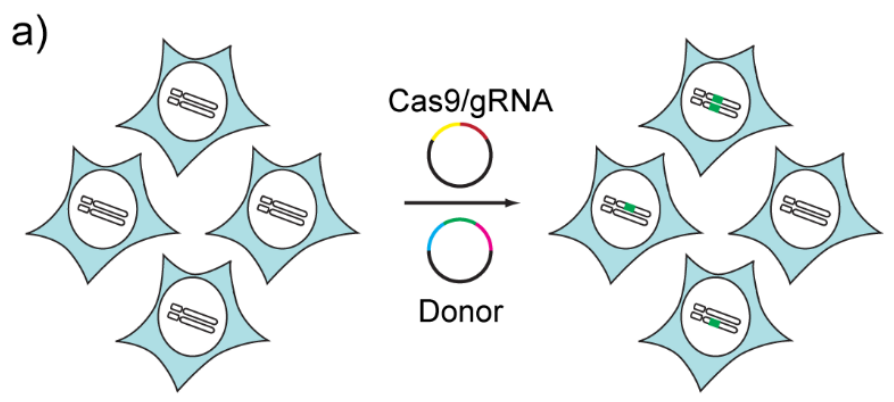

b)

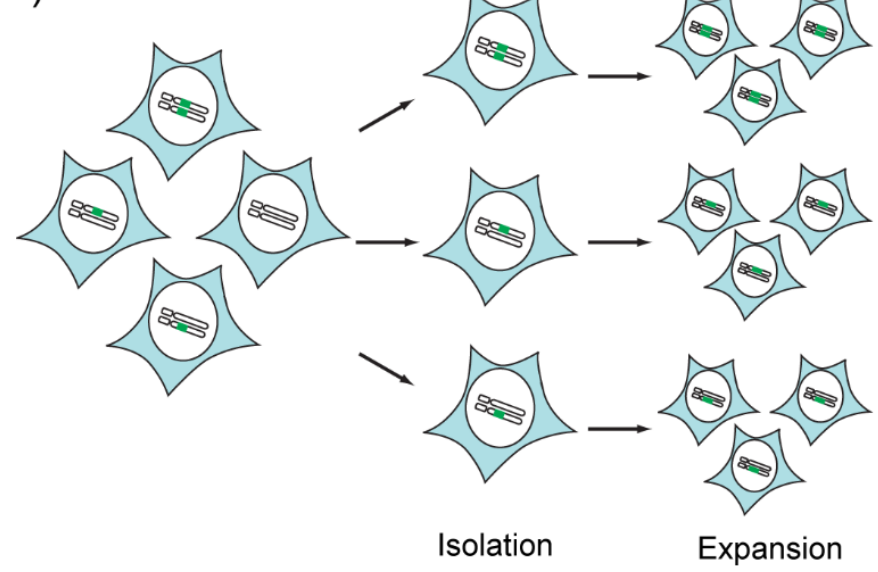

Figure 24: Clone selection and expansion. a) Co-transfection of Cas9/gRNA encoding plasmid and a donor plasmid carrying the transgene to be integrated yields a polyclonal mixture of targeted (heteroand homozygous clones) and non-targeted cells. b) Clone isolation is done by sorting single cells into 96-well plates using FACS. Expansion of single cells yields a monoclonal culture.

\subsubsection{Analysis of clonal cell lines}

Analysis of successfully targeted clonal cell lines was done using PCR, western blot (WB) and DNA sequencing of on- and off-target sites. Information about the zygosity of each cell line was obtained using out-out PCR. Here, locus-specific information about the transgene integration event is obtained by using two primers that anneal outside of the left and right homology arm of the donor plasmid. According to the resulting band pattern, the zygosity of a 
cell line can be easily evaluated: 1) a single band is detected for a wildtype clone, 2) a wildtype band and an about $700 \mathrm{bp}$ shifted single band are detected for a heterozygous clone and 3) a single about 700 bp shifted band with respect to the wildtype band is obtained for a homozygous clone (Figure 25a). If no suitable primers for an out-out PCR can be designed, an out-in or an in-out PCR can be used as well. Here the respective "in" primer anneals within the homology arms and the "out" primer anneals outside of them ensuring locus-specific analysis of transgene integration.

Information about the nature of the integrated transgene was obtained using junction PCR (jPCR). Here, a transgene-specific primer and a locus-specific primer are used to generate a PCR product across the junction of the transgene and the gene to be modified. A PCR product of characteristic size is obtained for both heterozygous and homozygous clones, but not for wildtype cells (Figure 25b).

a)

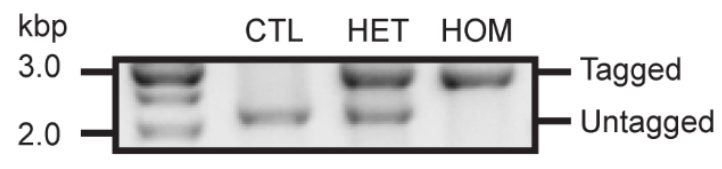

b)

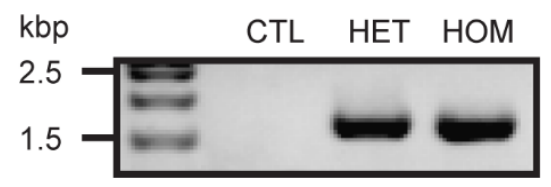

Figure 25: Genotyping of clonal cell lines. a) Out-out PCR analysis of monoclonal cell lines gives locus-specific information about a transgene integration event. b) Junction PCR (jPCR) gives information about the nature of the transgene at a specific locus. Anticipated results are shown for wild type (CTL), heterozygously tagged (HET) and homozygously tagged (HOM) cells. (Adapted from from Ratz et al., 2015).

To assess whether the generated fusion protein was stably expressed from the locus of interest, western blot analysis using protein-specific and transgene-specific antibodies was done. Information about presence and stability of both the wildtype and the fusion protein was obtained using protein-specific antibodies. The anticipated result corresponds to that of an out-out PCR: 1) a single band is detected for a wildtype clone, 2) a wildtype band and an about $27 \mathrm{kDa}$ shifted band with respect to it are detected for a heterozygous clone and 3) a single, about $27 \mathrm{kDa}$ shifted band with respect to the wildtype band is obtained for a homozygous clone (Figure 26a).

To assess whether the integrated GFP-transgene was stably expressed as a fusion protein, a GFP-specific antibody was used. The resulting band pattern corresponds to the pattern observed during jPCR: no band is detected for the wildtype unmodified protein whereas an 
about $27 \mathrm{kDa}$ shifted band with respect to the wildtype protein should be visible for both hetero- and homozygous clones (Figure 26b).

a)

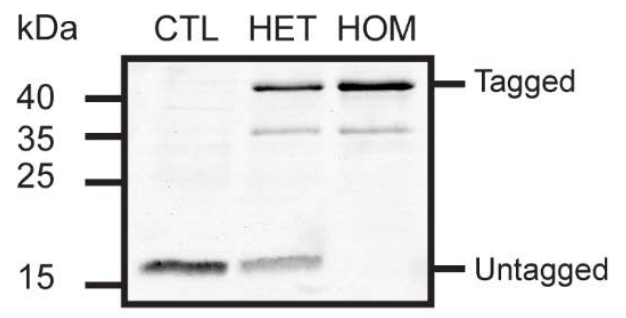

b)

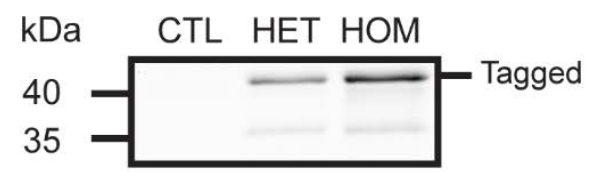

Figure 26: Immunoblotting of clonal cell lines. a) Analysis using a target protein specific antibody gives information about fusion protein stability and expression level. b) Analysis using a tag specific antibody gives information about site-specific integration. Anticipated results are shown for wild type $(\mathrm{CTL})$, heterozygously tagged (HET) and homozygously tagged (HOM) cells. (Adapted from from Ratz et al., 2015).

Finally, verification of site-specific transgene integration and analysis of potential Cas9induced off-target mutations was done using Sanger sequencing. To obtain locus-specific sequence information, out-out/out-in/in-out and junction PCR products were used. Potential off-target sites were predicted using the CRISPR Design Tool (Hsu et al., 2013; http://crispr.mit.edu). PCR products containing on- or off-target sites were used for TOPOcloning and subsequent Sanger sequencing.

\subsection{CRISPR-Cas9-mediated endogenous protein tagging for super- resolution imaging of living human cells}

The established general workflow for CRISPR-Cas9-mediated endogenous protein tagging was used for tag integration at various genomic loci in human cells. An overview about all 24 generated cell lines is given in Table 15. The following part describes the use of a selection of three endogenously rsEGFP2-tagged cell lines for live cell RESOLFT imaging.

\subsubsection{Endogenous tagging of HMGA1, VIM and ZYX with rSEGFP2}

Using the established workflow, tagging was done for the nuclear DNA-binding non-histone high mobility group protein HMG-I (gene: HMGA1), the class-III intermediate filament protein vimentin (gene: VIM), and the focal adhesions protein zyxin (gene: ZYX). These proteins were chosen because they exhibit different expression levels and are localized in different cellular compartments, i.e. the nucleus (HMG-I), the cytoskeleton (vimentin) and in the 
plasma membrane associated focal adhesion complexes (zyxin). Further, whether homozygous tagging could be carried out for various proteins had to be addressed.

For each of the three target genes, two guide RNAs (gRNAs) were designed that target the stop codon region of the respective gene (Figure 27). While tools for predicting gRNA efficiency are becoming increasingly popular, the actual gRNA activity is still somewhat unpredictable (Moreno-Mateos et al., 2015; Port et al., 2015). To circumvent this issue and to ensure that at least one gRNA will lead to efficient cleavage, two gRNAs were designed in silico and generated via oligo cloning.

a) VIM stop codon region

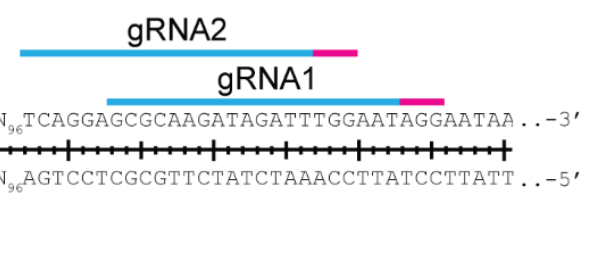

b) ZYX stop codon region

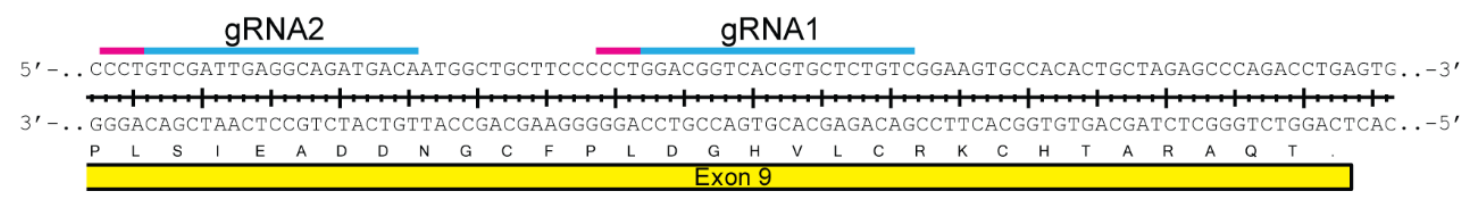

C) HMGA1 stop codon region

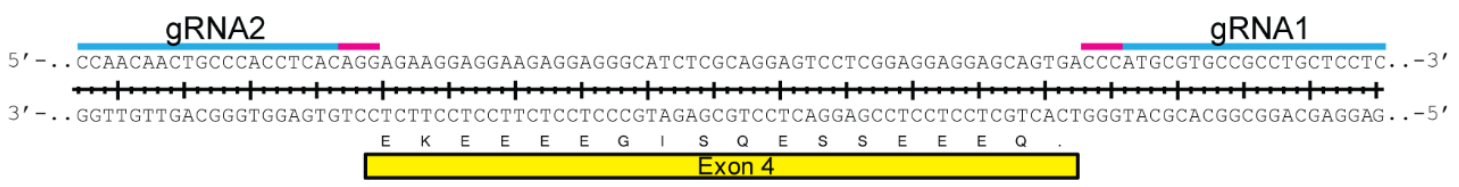

Figure 27: Design of gRNAs for targeting three human genes. Using the CRISPR Design Tool two gRNAs were designed to target the stop codon regions of the human genes encoding for vimentin (a), zyxin (b) or HMG-I (c), respectively. Each gRNA is targeting a 20bp nucleotide sequence (light blue) followed by an upstream protospacer-adjacent motif (PAM, magenta).

To facilitate the integration of the rsEGFP2 coding sequence at the 3'-end of the respective last exon, donor plasmids with homology between 590 and 924 bp were generated (Figure 28). The donor plasmid VIM-rsEGFP2 used for tagging vimentin (VIM) with rSEGFP2 was generated using four fragment cloning. Gibson assembly was used for generation of ZYXrSEGFP2 or HMGA1-rSEGFP2 donor plasmids for endogenous tagging of zyxin (ZYX) or HMG-I (HMGA1), respectively. 
a)

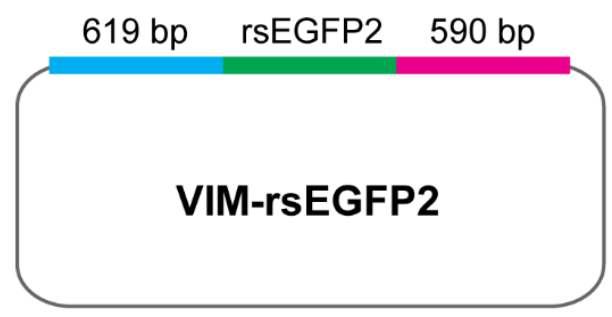

c)

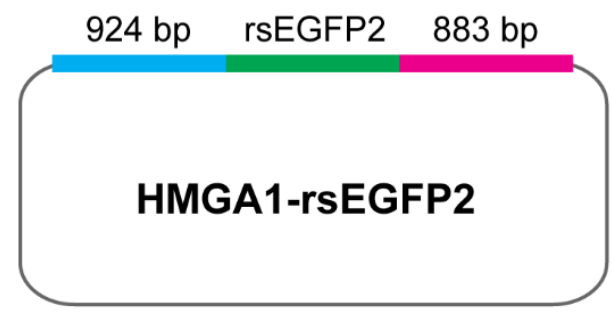

b)

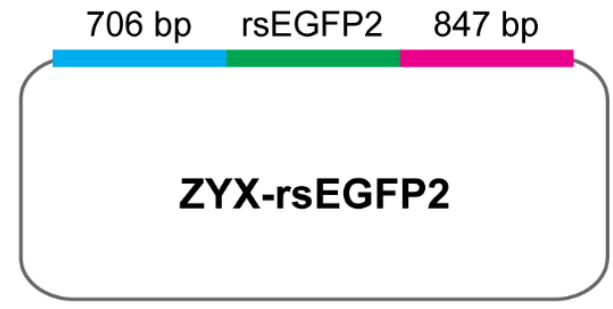

d)

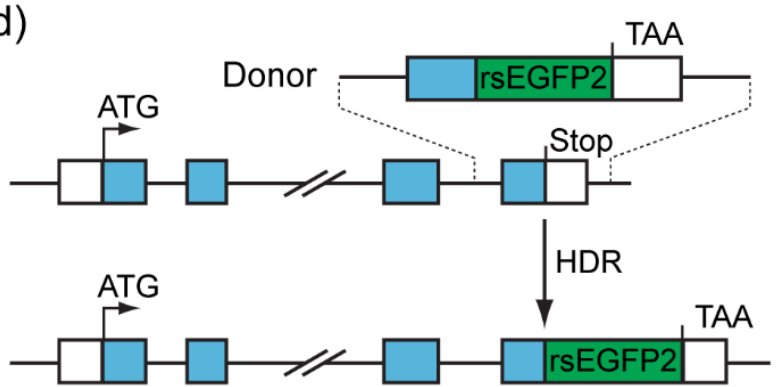

Figure 28: Donor plasmids for C-terminal tagging. a-c) Donor plasmids were generated for stop codon replacement by the rsEGFP2 coding sequence at the human VIM (a), ZYX (b) or HMGA1 (c) gene, respectively. Each plasmid was based on a pUC57 backbone and contained a left homology arm (light blue) and a right homology arm (magenta) of indicated length flanking the rsEGFP2 coding DNA. d) Homology-directed repair (HDR) leads to rsEGFP2 transgene integration and stop codon replacement at the respective genomic locus.

Human U2OS cells were co-transfected with the respective bicistronic gRNA/Cas9 plasmid and the corresponding donor matrix. Transfection with donor plasmid only served as a negative control to assess for potential random integration events in absence of a DSB. Seven days after transfection, cells were analyzed using fluorescence activated cell sorting (FACS). Mean integration efficiency of rsEGFP2 at the VIM locus was $0.77 \%$ or $0.73 \%$ using VIM-gRNA1 or VIM-gRNA2, respectively (Figure 29). The mean fraction of cells expressing zyxin-rsEGFP2 was $0.12 \%$ using ZYX-gRNA1 whereas ZYX-gRNA2 failed to generate rsEGFP2-positive cells above donor plasmid only level. HMGA1 tagging with rsEGFP2 was achieved on average in $4.72 \%$ of the cells using HMGA1-gRNA1 and in $2.23 \%$ using HMGA1-gRNA2, respectively. 
a)
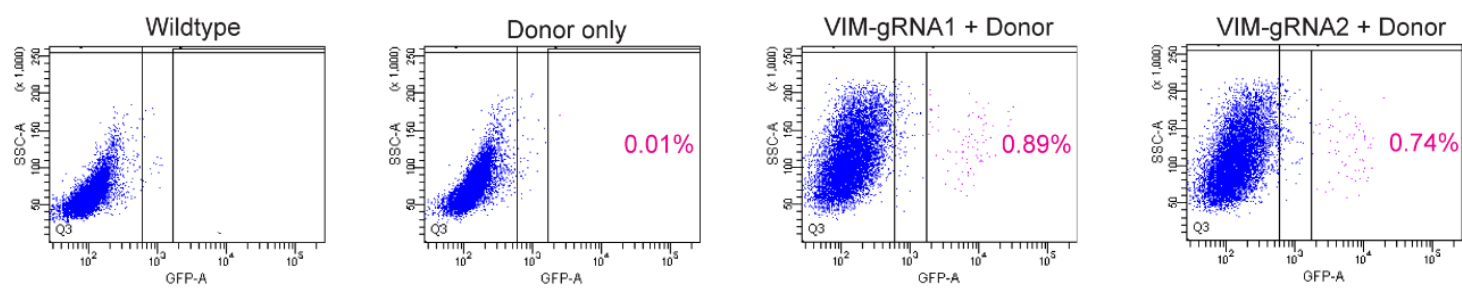

b)
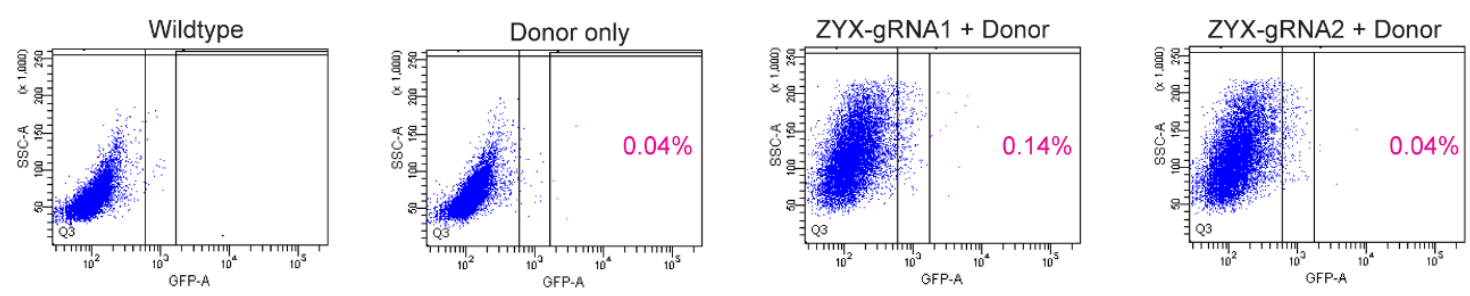

C)
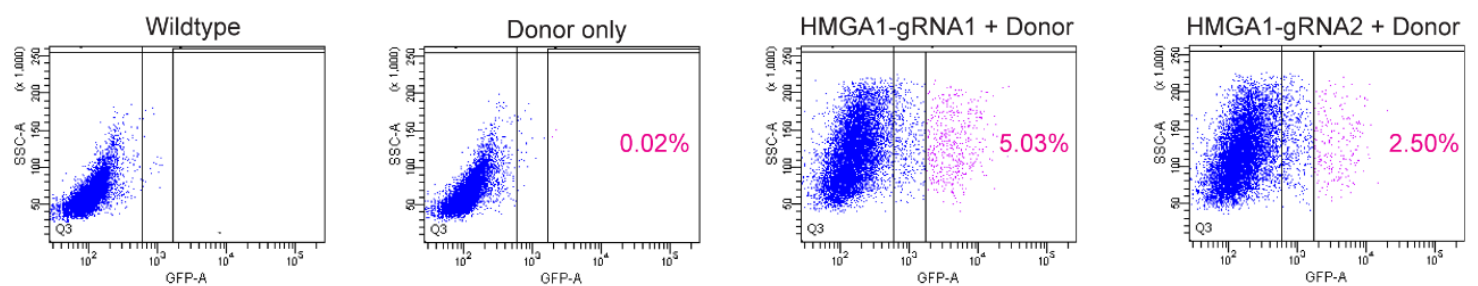

Figure 29: FACS analysis of U2OS cells after nuclease and donor plasmid transfection. Cells cotransfected with VIM-gRNA1/donor or VIM-gRNA2/ donor (a), ZYX-gRNA1/donor or ZYX-gRNA2/donor (b) and HMGA1-gRNA1/donor or HMGA1-gRNA2/donor (c) were analysed via flow cytometry. Wildtype U2OS cells served as a control to assess for cellular autofluorescence. Donor plasmid only transfected cells served as a control to assess for the fraction of cells generated via random integration events of the plasmid in absence of nuclease. Co-transfections were done in duplicates of which one representative dot plot is shown.

Single rsEGFP2-positive cells were sorted into 96-well plates per nuclease/donor combination. Within two weeks after sorting, between $10 \%$ and $20 \%$ of the sorted cells recovered and grew to confluency per plate. Those cells were detached from the growth surface and transferred to 24-well plates. After another week, cells were split again and replicated in a second 24-well plate containing cover slips for subsequent fluorescence microscopy analysis. Clones that expressed a fusion protein at the correct sub-cellular localization were further cultivated for analysis via out-out PCR (Figure 30). It should be noted that a few clones were highly fluorescent, but the signal was not restricted to any specific cellular structure. These clones probably represent the fraction of cells in which the donor plasmid underwent random genomic integration followed by FP expression.

Among the two zyxin-rsEGFP2 clones, one heterozygous and one homozygous knock-in cell line was identified. The nuclease/donor matrix pairs targeting the VIM locus resulted in 8 (VIM-gRNA1) or 9 (VIM-gRNA2) heterozygous monoclonal lines per 96-well plate, 
respectively. In case of HMG-I-rsEGFP2, one homozygous and five heterozygous monoclonal lines (HMGA1-gRNA1) were generated, while one homozygous and four heterozygous clones were obtained for cells treated with HMGA1-gRNA2.

a)

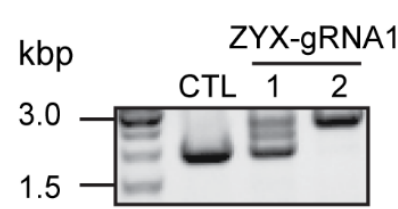

b)

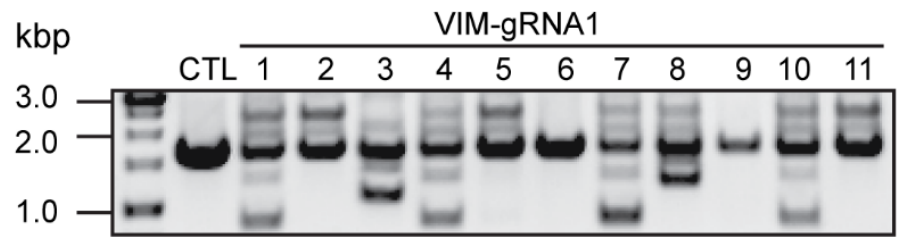

c)

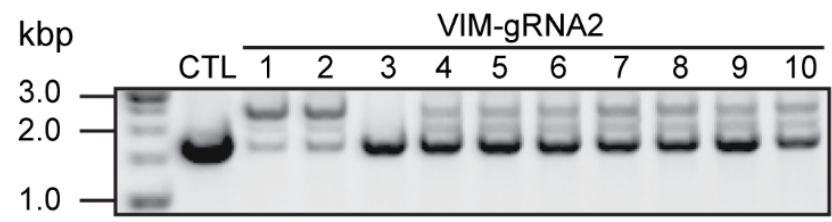

d)

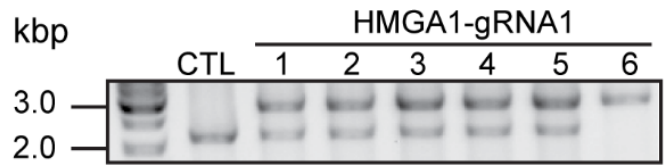

e)

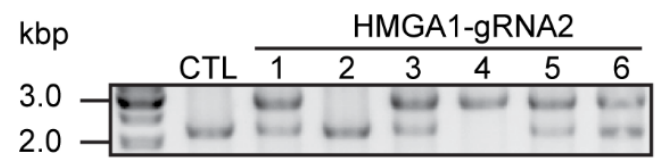

Figure 30: out-out PCR analysis of knock-in cells. Out-out PCR was used for genotyping of monoclonal cell lines obtained after single cell sorting. Genotypting results are shown for zyxinrSEGFP2 clones generated with ZYX-gRNA1 (a), vimentin-rsEGFP2 clones generated with VIMgRNA1 (b) or VIM-gRNA2 (c) and HMG-I-rsEGFP2 cell lines generated with HMGA1-gRNA1 (d) or HMGA1-gRNA2 (e).

In summary, for the three genes targeted between 2 and 9 endogenously tagged cell lines were obtained per 96-well plate. Except for tagging of VIM with rsEGFP2, one homozygous clone per nuclease/donor combination was obtained for ZYX and HMGA1, respectively. An overview of these results is given in Table 1. 
Table 1: Generated knock-in cell lines. For each nuclease/donor pair, the fraction of cells exhibiting rsEGFP2 fluorescence (rsEGFP2+) was determined by FACS analysis of about 10,000 cells. The given numbers of genotyped clones refer to the analysis of clones obtained from a single 96-well plate.

\begin{tabular}{lccccc}
\hline Target & gRNA & rsEGFP2+ (\%) & Genotyped & Homozygous & Heterozygous \\
\hline \multirow{2}{*}{ VIM } & 1 & 0.77 & 11 & - & 8 \\
\cline { 2 - 6 } & 2 & 0.73 & 10 & - & 9 \\
\hline \multirow{2}{*}{ ZYX } & 1 & 0.12 & 2 & 1 & 1 \\
\cline { 2 - 6 } & 2 & 0.06 & - & - & - \\
\hline \multirow{2}{*}{ HMGA1 } & 1 & 4.72 & 6 & 1 & 5 \\
\cline { 2 - 6 } & 2 & 2.23 & 6 & 1 & 4 \\
\hline
\end{tabular}

To take potential off-target effects into account, one clone per Cas9/gRNA pair was selected. This strategy is generally employed in order to ensure that a phenotype does not arise due to off-target mutagenesis of one specific gRNA. Instead, the observation of the same phenotype created with multiple gRNAs indicates that the phenotype is due to an on-target effect (KoikeYusa et al., 2014; Shalem et al., 2014; Wang et al., 2014a). Therefore, one homozygous and one heterozygous clone were picked from the obtained endogenously rsEGFP2-tagged clones for further experiments.

Genotyping of the selected clones was done via out-out PCR and junction PCR (Figure 31). Out-out PCR confirmed the heterozygosity of clones HMGA1-rsEGFP2-HET, ZYX-rsEGFP2HET as well as VIM-rsEGFP2-HET1 and VIM-rsEGFP2-HET2. Further, a single PCR product band was detected for the clones HMGA1-rsEGFP2-HOM and ZYX-rsEGFP2-HOM indicating that those cell lines are homozygous for rsEGFP2 integration at the respective locus.

a)

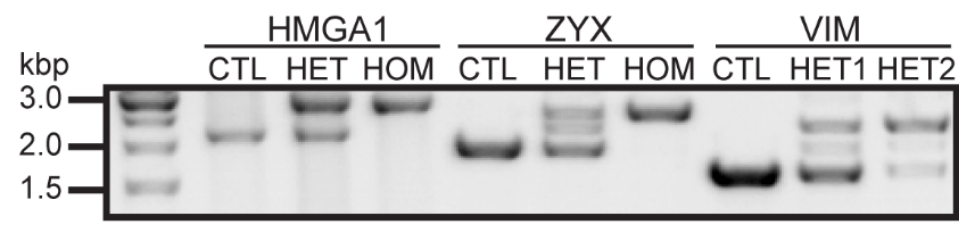

b)

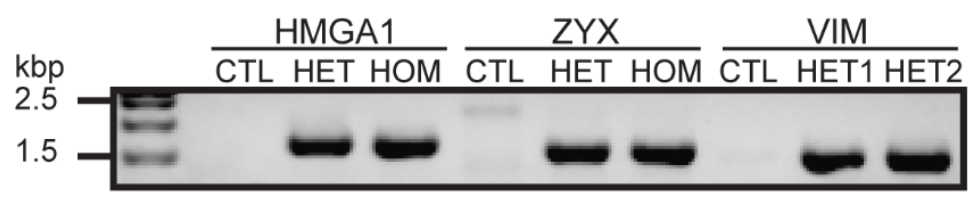

Figure 31: Genotyping of selected knock-in cells. a) Out-out PCR analysis using a primer pair annealing outside the homology arms. b) Junction PCR analysis using a forward primer that anneals outside the left homology arm and a reverse primer that anneals within the rsEGFP2 coding sequence. CTL, control (parental U2OS cells); HMGA1-HET, heterozygous HMGA1-rsEGFP2-HET1.5 clone, 
HMGA1-HOM, homozygous HMGA1-rsEGFP2-HOM2.4 clone; ZYX-HET, heterozygous ZYX-rsEGFP2 clone; ZYX-HOM, homozygous ZYX-rsEGFP2-HOM clone; VIM-HET1, heterozygous VIM-rsEGFP2HET1.2 clone; VIM-HET2, heterozygous VIM-rsEGFP2-HET2.1 clone.

Analysis of (fusion) protein expression levels and stability was done using immunoblotting. Wildtype U2OS cells express HMG-I with a size of $12 \mathrm{kDa}$, whereas heterozygous knock-in cells express both untagged HMG-I and HMG-I-rsEGFP2 with a molecular weight (MW) of about 40 kDa (Figure 32a). Homozygous knock-in cells express tagged HMG-I-rsEGFP2 only. Note that for both knock-in cell lines a shorter HMG-I-rsEGFP2 fusion with an apparent MW of about $35 \mathrm{kDa}$ could be detected but not for the wildtype U2OS cells. Unmodified U2OS cells express zyxin with a predicted MW of $50 \mathrm{kDa}$ and an observed MW of about 80 $\mathrm{kDa}$ (Figure 32b). This size difference is well explained by extensive posttranslational modifications of zyxin observed experimentally (Choudhary et al., 2009; Olsen et al., 2006). As expected, the heterozygous zyxin-rsEGFP2 clone shows a second band with a MW of about $110 \mathrm{kDa}$ indicating stable expression of zyxin-rsEGFP2. Here, untagged zyxin also runs at a higher MW explained by the fact that ZYX-gRNA1 induces mutations within the last exon of the gene leading to a modified but untagged protein (see below). U2OS cells homozygous for an rsEGFP2 knock-in at the ZYX locus only express tagged zyxin-rsEGFP2. Both heterozygous vim-rsEGFP2 clones express wildtype vimentin from the unmodified allele and vimentin-rsEGFP2 from the modified allele (Figure 32c).

a)

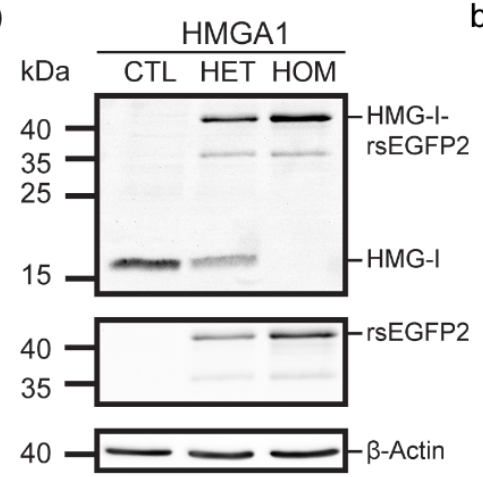

b)

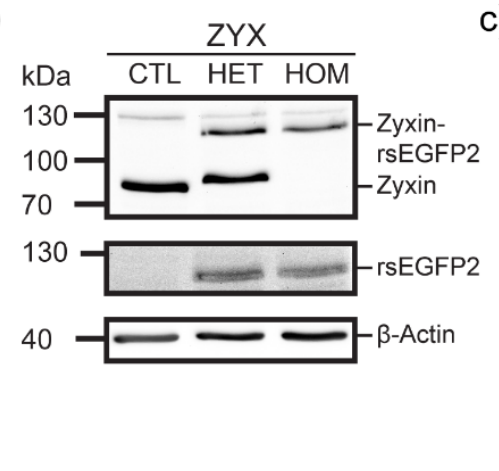

C)

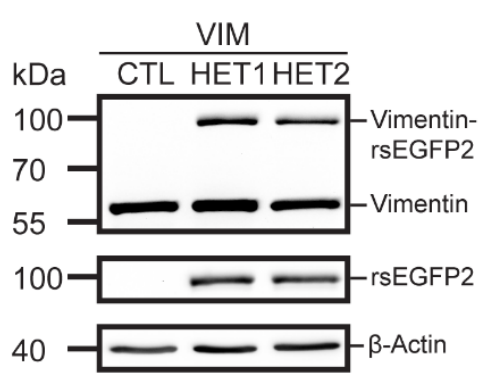

Figure 32: Western blot analysis of selected cell lines. Cell lysates of monoclonal cell lines were immunoblotted for rSEGFP2, beta-Actin and the respective endogenously tagged protein: HMG-I (a), zyxin (b) and vimentin (b).

In summary, each of the selected cell lines incorporated the coding sequence of rsEGFP2 at the specified locus. Further, the rsEGFP2 fusion proteins are expressed stably from the respective locus leading to a mixed population of both tagged and untagged protein for the heterozygous and to a pure population of tagged protein for the homozygous knock-in cells. 


\subsubsection{DNA sequencing of endogenously tagged cell lines}

\subsubsection{On-target site sequencing}

Each gRNA was targeted towards a specific gene sequence of interest, the on-target site. Additionally, each gRNA potentially targets a number of off-target sites within the genome of interest leading to unintended mutations and unwanted chromosomal translocations (Wu et al., 2014). The next section presents the analysis of both on- and off-target sites using Sanger sequencing of TOPO-cloned PCR products.

Both VIM-targeting nucleases (VIM-gRNA1 and 2) are expected to induce a DSB within the 3' UTR of the gene close to the stop codon. Consequently, no mutations of the coding sequence were observed. However, both rsEGFP2-tagged alleles and untagged alleles exhibited small deletions in the non-coding regions where the respective nuclease induces DNA cleavage (Figure 33).

a)

$$
\begin{aligned}
& \text { Untagged } \\
& \text { VIM-rSEGFP2-HET1 }
\end{aligned}
$$

b)

$$
\begin{aligned}
& \text { Tagged } \\
& \text { VIM-rSEGFP2-HET1 }
\end{aligned}
$$

c)

$$
\begin{aligned}
& \text { Untagged } \\
& \text { VIM-rSEGFP2-HET2 }
\end{aligned}
$$

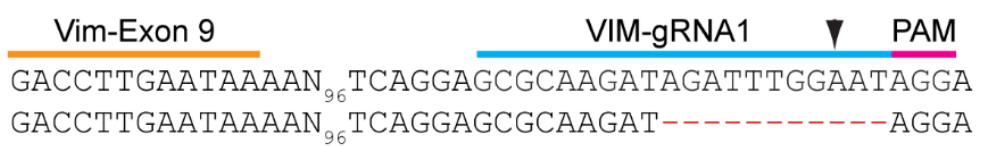

$$
\begin{aligned}
& \frac{\text { rsEGFP2 }}{\text { TACAAGTAACCN }}{ }_{102} \text { TCAGGAGCGCAAGATAGATTTGGAATAGGA } \\
& \text { TACAAGTAACCN }{ }_{102} \text { TCAGGAGCGCAAGATAGATTTG-AATAGGA }
\end{aligned}
$$

d)

$$
\begin{aligned}
& \text { Tagged } \\
& \text { VIM-rSEGFP2-HET2 }
\end{aligned}
$$

Figure 33: On-target sequencing of vimentin-rsEGFP2 clones. $a, b)$ VIM-gRNA1 induced an 11 bp deletion in the untagged allele (a) and a 1 bp deletion in the tagged allele of clone VIM-rsEGFP2HET1.2. c, d) VIM-gRNA2 induced a 2 bp deletion in the untagged (c) and a 3 bp deletion in the tagged (d) allele of clone VIM-rSEGFP2-HET2.1. Wildtype reference sequences for untagged and tagged alleles are given above the actual consensus Sanger sequencing results. 
Particularly, the untagged allele of the first heterozygous clone Vim-rsEGFP2-HET1 contained an 11 bp deletion whereas the tagged allele carried a 1 bp deletion (Figure 33a, b). The untagged allele of the second clone Vim-rSEGFP2-HET2 contained a 2 bp deletion whereas the tagged allele carried a 3 bp deletion (Figure 33c, d). Interestingly, all mutations induced sequence alterations in the DNA strand complementary to the 'seed region' of the gRNA. The seed region comprises the last 12 nucleotides of the gRNA and mismatches between gRNA and DNA in this region are known to efficiently abrogate Cas9 cleavage activity (Hsu et al., 2013). The presented results confirm the low mismatch tolerance in this region as already a 1 bp deletion efficiently inhibits further Cas9-mediated DNA cleavage.

To modify the HMGA1 gene, one nuclease was guided to the 3' UTR (HMGA1-gRNA1) with the PAM site directly flanking the stop codon, while the second nuclease targeted the last intron (HMGA1-gRNA2). As expected no large insertions or deletions occurred that would affect nearby coding sequences (Figure 34). However, each nuclease target site contained small DNA sequence modifications. Again, those mutations were found in the DNA sequence required for hybridization to the gRNA seed region, highlighting the low tolerance of gRNAs to mismatches in this region. Specifically, a 1 bp insertion was found in the untagged allele of the heterozygous clone (Figure 34a) while a larger deletion of 11 bp was detected at the allele containing the rSEGFP2 coding sequence (Figure 34b). Unexpectedly, both of the tagged alleles in the homozygous HMGA1-rsEGFP2 clone exhibit an identical consensus sequence containing a 1 bp insertion (Figure 34c). This was surprising, because DSB repair via non-homologous end joining (NHEJ) is characterized by random addition or deletion of nucleotides that results in a diverse pattern of mutations (Cradick et al., 2013). As a homozygous knock-in cell is assumed to result from two independent donor plasmid integration events at two alleles, further Cas9-mediated mutagenesis at those alleles should lead to two different mutation patterns. However, an alternative explanation is that only one allele recombined with the provided donor plasmid initially followed by a secondary mutation that abrogates the gRNA binding site in the donor template. The now 'Cas9-resistant' allele could well serve as a repair template for the second allele thereby converting an initially heterozygous mutation to homozygosity. In fact, a similar mechanism known as the 'mutagenic chain reaction' is employed to convert heterozygous flies to homozygosity (Gantz and Bier, 2015). 
a)

Untagged

HMGA1-rSEGFP2-HET
Exon 4 PAM $\quad$ HMGA1-gRNA1

GAGCAGTGACCCATG-CGTGCCGCCTGCTCCTCACTGGAGGAGC

GAGCAGTGACCCATGTCGTGCCGCCTGCTCCTCACTGGAGGAGC

ISEGFP2 PAM \ HMGA1-gRNA1

TACAAGTAA GGTACCDCCATGCGTGCCGCCTGCTCCTCACTGGAGG

TACAAGTAAGGTACCCCCA---n---n--TGCTCCTCACTGGAGG

c)

Tagged

HMGA1-gRNA2 \ PAM

rsEGFP2

HMGA1-rSEGFP2-HOM

Figure 34: On-target sequencing of HMG-I-rsEGFP2 clones. a, b) HMGA1-gRNA1 induced a 1 bp insertion in the untagged allele (a) and an $11 \mathrm{bp}$ deletion in the tagged allele of clone HMG-I-rsEGFP2HET1.5. c) All tagged alleles of the homozygous clones HMG-I-rsEGFP2-HOM2.4 carry a 1 bp insertion induced by HMGA1-gRNA2. Wildtype reference sequences for untagged and tagged alleles are given above the actual consensus Sanger sequencing results.

The active ZYX-specific nuclease (ZYX-gRNA1) was targeted to the terminal exon for induction of a DSB. To avoid secondary nuclease-mediated mutations in the coding region after transgene integration, silent mutations within the nuclease binding site in the donor plasmid were introduced. As expected, this strategy successfully prevented Cas9-induced mutagenesis in alleles that recombined with the 'nuclease-resistant' donor plasmid (Figure 35b, c). However, DNA sequencing revealed that NHEJ induced the deletion of a single cytosine in the non-tagged allele of the heterozygous ZYX-rsEGFP2 cell line (Figure 35a). This single point mutation in the terminal exon resulted in a frameshift that ultimately led to the expression of a mutated version non-tagged zyxin. 
a)

Untagged

ZYX-rSEGEP2-HET

Untagged

ZYX-rSEGEP2-HET

b)

Tagged

ZYX-rSEGEP2-HET

c)

Tagged

ZYX-rSEGFP2-HOM
PAM \ZYX-gRNA1

CCCCCTGGACGGTCACGTGCTCTGTCGGAAGTGCCACACTGCTAG

CCCCCTGGA-GGTCACGTGCTCTGTCGGAAGTGCCACACTGCTAG

Protein mutation

DCGKPLS IEADDNGCEPLDGHVLCRKCHTARAQT- .

DCGKPLS IEADDNGCFPLEVTCSVGSATLLEPRPE.

PAM \ ZYX-gRNA1

CCCTCTGGATGGTCATGTGCTCTGTCGGAAGTGCCACACTGCTAG

CCCTCTGGATGGTCATGTGCTCTGTCGGAAGTGCCACACTGCTAG

PAM $\quad$ ZYX-gRNA1

CCCTCTGGATGGTCATGTGCTCTGTCGGAAGTGCCACACTGCTAG

CCCTCTGGATGGTCATGTGCTCTGTCGGAAGTGCCACACTGCTAG

Figure 35: On-target sequencing of zyxin-rsEGFP2 clones. a) ZYX-gRNA1 induced a 1 bp deletion within the last exon of $Z Y X$ leading to change of the amino acid sequence in the expressed protein. $b$, c) ZYX-gRNA1 induced mutations within the tagged allele of clone ZYX-rsEGFP2-HET (b) and clone ZYX-rsEGFP2-HOM (c) are avoided after donor plasmid integration carrying silent mutations in the nuclease binding site (red characters). Wildtype reference sequences for untagged and tagged alleles are given above the actual consensus Sanger sequencing results.

In summary, DNA sequencing of gRNA/Cas9 on-target sites revealed some interesting aspects about the CRISPR system. First, Cas9-induced DSBs mainly result in small indels that range from 1-11 bps, but no major genomic rearrangements occur. Second, all mutations were found in the gRNA seed region, highlighting the sensitivity of this region towards mismatches. Finally, silent mutations in the gRNA binding site and the adjacent PAM can be introduced into donor plasmids to efficiently inhibit Cas9-mediated mutagenesis after transgene integration. As this strategy prevents donor plasmid degradation by Cas9 nuclease activity, 'nuclease-resistant' repair templates should be used for subsequent experiments relying on homologous recombination.

\subsubsection{Off-target site sequencing}

Next to on-target site analysis, potential off-target site mutations in each clone were assessed. Therefore, the top two off-target sites as predicted with the CRISPR Design Tool were PCR amplified and fragments containing the respective site were sub-cloned for Sanger sequencing. An overview of the two off-target DNA sequences with the highest similarity to the on-target sequence for each nuclease is given in Table 2. 
Table 2: Overview of the top two predicted off-target sites associated with each gRNA. Off-target sites were predicted using the CRISPR Design Tool. Given are the off-target (OT) sequence, the number of mismatches as well as their position with respect to the on-target site. Chromosome number, nucleotide position on that chromosome and the corresponding gene, specifies the off-target locus. nc: non-coding DNA region.

\begin{tabular}{llllll}
\hline gRNA & OT & OT sequence (5' to 3') & Mismatches & Locus & Gene \\
\hline VIM- & 1 & ACTCAAGAAAGATTTGGAATGAG & $3[1: 3: 9]$ & chr5:+45464916 & $\mathrm{nc}$ \\
gRNA1 & 2 & GTTTAAGCTAGATTTGGAATTGG & $4[2: 3: 4: 8]$ & chr21:-27524959 & $\mathrm{nc}$. \\
\hline VIM- & 1 & TCTGGAGAGAAAGATAGATTTGG & $3[3: 8: 10]$ & chr6:+114646632 & $\mathrm{nc}$ \\
gRNA2 & 2 & TCAAGAGAGGAAGATAGATTAGG & $3[4: 8: 10]$ & chr2:+170477087 & $\mathrm{nc}$ \\
\hline ZYX- & 1 & GAGAGAGCAGGTGACCCTCCTGG & $3[3: 10: 17]$ & $\mathrm{chr} 16:-11937364$ & $\mathrm{nc}$ \\
gRNA1 & 2 & GTTTGAGCACGTGACCTTCCAAG & $4[2: 3: 4: 17]$ & $\mathrm{chr} 18:+31739510$ & $\mathrm{nc}$ \\
\hline HMGA1- & 1 & GACCAGCAGGCGGCACTCATCAG & $3[3: 4: 17]$ & $\mathrm{chr}:+153312911$ & PGLYRP4 \\
gRNA1 & 2 & AGGGAGCAGCAGGCACGCATTAG & $4[1: 2: 10: 11]$ & $\mathrm{chr} 1:+57315588$ & $\mathrm{nc}$ \\
\hline HMGA1- & 1 & AAAGCAACTGCCCACCTCACAAG & $3[1: 2: 4]$ & chr5:+132169640 & $\mathrm{nc}$ \\
gRNA2 & 2 & ACAAAAGCTGCCCACCTCACAAG & $3[1: 5: 7]$ & chr4:+73414666 & ADAMTS3 \\
\hline
\end{tabular}

Endogenous tagging of the VIM gene was carried out by using VIM-gRNA1 or VIM-gRNA2 to guide Cas9 to the stop codon of the gene. The top two off-target sites for each gRNA are predicted to lie within non-coding regions of chromosomes 5 and 21 (VIM-gRNA1) as well as chromosomes 6 and 2 (VIM-gRNA2), respectively. However, neither the Vim-rsEGFP2 clone generated with gRNA1 nor the Vim-rsEGFP2 cell line generated with gRNA2 showed any Cas9-induced alterations at those sites (Figure 36). This was expected as all off-target sites exhibit a) 3-4 nucleotide mismatches between the potential gRNA:DNA duplex and b) 1-2 of those mismatches are found in the 12 bp seed region upstream of the PAM that is crucial for high Cas9 binding and cleavage activity (Hsu et al., 2013; Ran et al., 2013b). 
a)

WT

VIM-rSEGFP2-HET1.2

b)

WT

VIM-rSEGFP2-HET1.2

c)

WT

VIM-rsEGFP2-HET2.1

d)

WT

VIM-rSEGFP2-HET2.1
TTAAAACTTTACTCAAGAAAGATTTGGAATGAGAAGGCTA

TTAAAACTTTACTCAAGAAAGATTTGGAATGAGAAGGCTA

OT2: VIM-gRNA1 \ PAM GTAAAATTATGTTTAAGCTAGATTTGGAATTGGGAAAGAA GTAAAATTATGTTTAAGCTAGATTTGGAATTGGGAAAGAA

TTGTTAGGTGTCTGGAGAGAAAGATAGATTTGGGATATAA TTGTTAGGTGTCTGGAGAGAAAGATAGATTTGGGATATAA

OT2: VIM-gRNA2 \ PAM

AGATGGCAATTCAAGAGAGGAAGATAGATTAGGAAACTTT AGATGGCAATTCAAGAGAGGAAGATAGATTAGGAAACTTT

Figure 36: Off-target site sequencing in vimentin-rsEGFP2 clones. None of the top two predicted off-target (OT) sites for VIM-gRNA1 $(a, b)$ or VIM-gRNA2 $(c, d)$ in the respective clones was modified.

HMGA1-gRNA1 is predicted to guide Cas9 to the gene coding for peptidoglycan recognition protein 4 (PGLYRP4) on chromosome 1 and to a non-coding region on the same chromosome for DSB induction. The top two off-target sites associated with HMGA1-gRNA2 are predicted to be a non-coding sequence on chromosome 5 and the gene coding for the protein 'A disintegrin and metalloproteinase with thrombospondin motifs 3' (ADAMTS3) on chromosome 4. Also, none of those sites showed any DNA modifications compared to wildtype cells (Figure 37). This was not surprising for HMGA1-gRNA1 as it fulfils all requirements for a high quality gRNA, i.e. at least three mismatches between the gRNA and the potential off-target site of which one mismatch is found within the gRNA seed region (Hsu et al., 2013; Ran et al., 2013b). However, HMGA1-gRNA2 does only contain three nucleotide mismatches within the first 8 bp or the 'non-seed region' that is significantly less mismatchsensitive. Absence of off-target mutagenesis for HMGA1-gRNA2 highlights that, next to the number and position of mismatches, the spacing between mismatched nucleotides is important. In fact, DNA sequences that contained nucleotide mismatches spaced less than four bases were not targeted by the respective gRNA (Hsu et al., 2013). Moreover, all predicted off-target sites for HMGA1-gRNA1 and HMGA1-gRNA2 contain a PAM site with the sequence NAG. While Cas9 cleaves targets sites containing 5'-NAG PAMs, the efficiency is one-fifth of the efficiency for target sites with 5'-NGG PAMs (Hsu et al. 2013). 
a)

WT

b)

WT

HMGA1-rSEGFP2-HET1. 5

c)

WT
HMGA1-rSEGFP2-HOM2 4

d)

WT

HMGA1-rSEGFP2-HOM2 . 4
OT1: HMGA1-gRNA1 \ PAM

GGATGTCCCGGACCAGCAGGCGGCACTCATCAGAAATGTT

GGATGTCCCGGACCAGCAGGCGGCACTCATCAGAAATGTT

OT2: HMGA1-gRNA1 PAM

AGTATGAAGTAGGGAGCAGCAGGCACGCATTAGCCCATTC

AGTATGAAGTAGGGAGCAGCAGGCACGCATTAGCCCATTC

OT1: HMGA1-gRNA2 \ PAM

ACACCAAAGCAAAGCAACTGCCCACCTCACAAGTCCCTGT

ACACCAAAGCAAAGCAACTGCCCACCTCACAAGTCCCTGT

OT2: HMGA1-gRNA2 \ PAM

CATAACACAGACAAAAGCTGCCCACCTCACAAGACCATAC

CATAACACAGACAAAAGCTGCCCACCTCACAAGACCATAC

Figure 37: Off-target site sequencing in HMG-I-rsEGFP2 clones. None of the top two predicted offtarget (OT) sites for HMGA1-gRNA1 (a, b) or HMGA1-gRNA2 (c, d) in the respective clones was modified.

Finally, the two top off-target sites for ZYX-gRNA1 are predicted to lie within non-coding regions of chromosomes 16 and 18. Again, neither the hetero- nor the homozygous clone generated with the help of ZYX-gRNA1 contained any off-target mutations at those sites (Figure 38). This is in agreement with the previous observations where 3-4 mismatches in the right position efficiently reduced Cas9 activity on a similar DNA sequence.

a)

WT

ZYX-rSEGFP2-HET

b)

WT
ZYX-rsEGFP2-HET

c)

WT

ZYX-rSEGFP2-HOM

d)

WT
ZYX-rSEGFP2-HOM
OT1: ZYX-gRNA1 \ PAM

TAGAACTATA GAGAGAGCAGGTGACCCTCCTGGAGGTAGG

TAGAACTATAGAGAGAGCAGGTGACCCTCCTGGAGGTAGG

GTCACGCCGAGTTTGAGCACGTGACCTTCCAAGAGCTTGA GTCACGCCGAGTTTGAGCACGTGACCTTCCAAGAGCTTGA

$\frac{\text { OT1: ZYX-gRNA1 } \quad \text { PAM }}{\text { TAGAACTATAGAGAGAGCAGGTGACCCTCCTGGAGGTAGG }}$
TAGAACTATAGAGAGAGCAGGTGACCCTCCTGGAGGTAGG

OT2: ZYX-gRNA1 \ PAM

GTCACGCCGAGTTTGAGCACGTGACCTTCCAAGAGCTTGA GTCACGCCGAGTTTGAGCACGTGACCTTCCAAGAGCTTGA

Figure 38: Off-target site sequencing in zyxin-rsEGFP2 clones. a-d) None of the top two predicted off-target (OT) sites for ZYX-gRNA1 was modified in the heterozygous $(a, b)$ or the homozygous (c, d) knock-in clone. 
In summary, no off-target cleavage activity was detected for the analyzed DNA sequences. This was expected as all of the used gRNAs fulfil at least one of the critical points for high target specificity: 1) number of mismatches is at least 3 nucleotides, 2) at least one mismatch lies within the PAM proximal region and 3) a maximal number of mismatches is spaced less than four bases (Hsu et al., 2013). Therefore, design of only two gRNAs based on the CRISPR Design Tool is sufficient to generate highly active and specific gRNAs useful for CRISPR-based endogenous protein tagging.

\subsubsection{Flow cytometry of endogenously tagged and overexpressing cells}

Fluorescence activated cell sorting (FACS) was applied to analyze rsEGFP2 fusion protein expression levels in living cells. FACS is capable to measure fluorophore concentrations in thousands of cells within a few seconds. Therefore, the method is a suitable tool for investigating the fusion protein expression levels between different samples.

FACS analysis of U2OS cells after vim-rsEGFP2 plasmid transfection revealed that $25.5 \%$ of the cells were fluorescent (Figure 39a). Within this fraction, the mean fluorescence intensity (MFI) per single cell varied substantially within the range of $90423 \pm 92271 \mathrm{AU}(\mathrm{SD}, \mathrm{n}=3819$ cells). The high standard deviation indicates that plasmid-based overexpression of vimrsEGFP2 leads to a large variation in fusion protein expression levels from cell-to-cell. This is in agreement with previous studies that showed a high variability in plasmid uptake per cell which ultimately results in highly heterogeneous protein expression levels (Cohen et al., 2009). On the other hand, FACS of endogenously tagged vim-rsEGFP2 knock-in cells showed that virtually every cell is fluorescent. Moreover, the measured MFI was $16263 \pm$ $9839 \mathrm{AU}$ (SD, $n=11800$ cells) for the first clone vim-rsEGFP2-HET1 and $16577 \pm 8320 \mathrm{AU}$ (SD, $n=11536$ cells) for the second clone vim-rsEGFP2-HET2. As both clones are heterozygous for rsEGFP2 integration at the VIM locus, the fluorescent intensities are expected to be similar. It is also not surprising, that overexpression of vim-rsEGFP2 from a heterologous promoter leads to artificially high fusion protein levels. In particular, overexpression levels are increased up to 23-fold compared to endogenous vim-rsEGFP2 expression levels. Interestingly, a high standard deviation for the MFI was also observed in endogenously tagged vim-rsEGFP2 cells. This indicates that, although individual cells were genetically identical, they exhibit substantial heterogeneity in fusion protein expression levels. Those expression level variations on a single cell level might be attributed to variations in gene expression, cell size and/or cell cycle stage (Kaern et al., 2005). 
a)

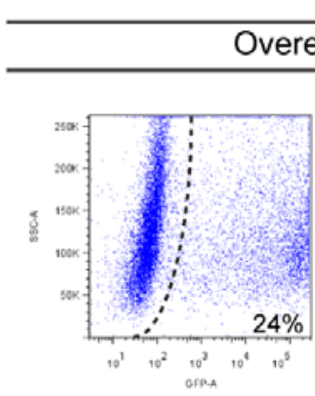

Vim-rsEGFP2

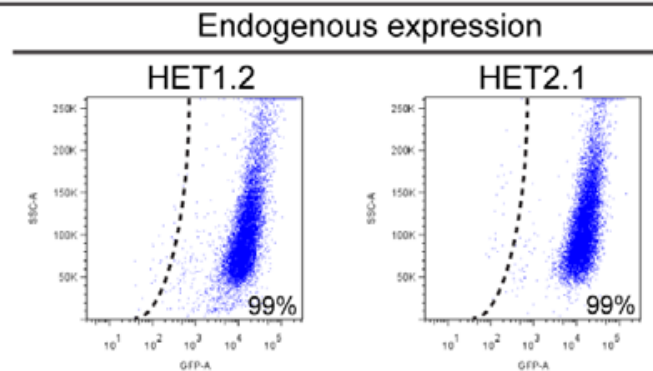

b)

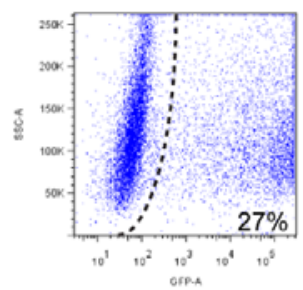

Zyx-rsEGFP2

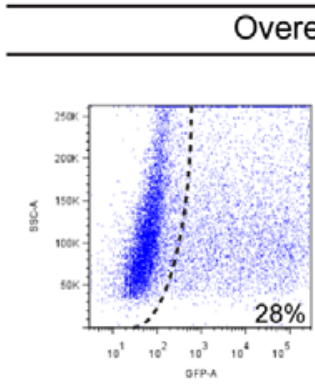

Overexpression
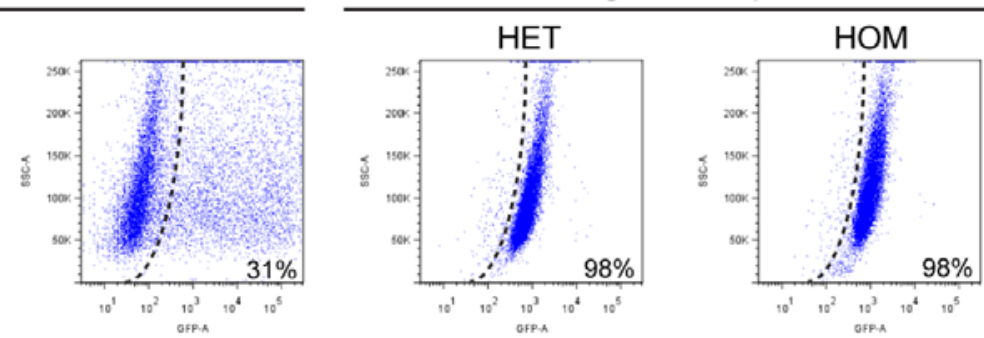

c)

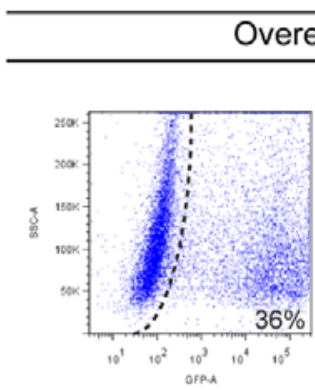

HMGA1-rsEGFP2
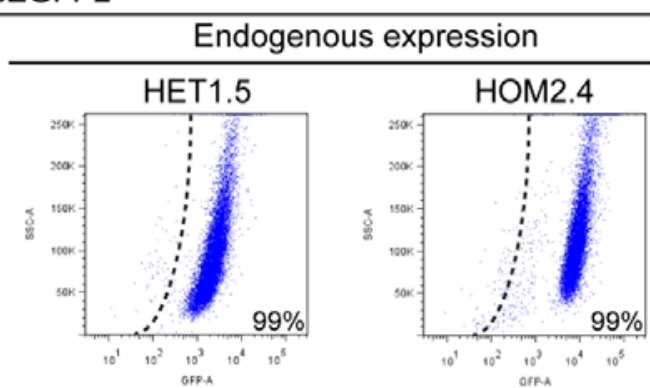

Figure 39: FACS of transiently transfected and stable knock-in cells. Comparison of transient overexpression and stable endogenous expression of vimentin-rsEGFP2 (a), zyxin-rsEGFP2 (b) and HMG-I-rsEGFP2 (c) fusion proteins. Overexpression experiments were done in duplicates. Fraction of rsEGFP2+ (\%) cells is given in each dot plot. (Adapted from Ratz et al., 2015).

Transfection of U2OS cells with a plasmid coding for zyx-rsEGFP2 resulted in $29.5 \%$ fluorescent cells on average (Figure 39b). On a single cell level, a large variation in MFI could be detected that exhibited values within $37793 \pm 60852$ AU (SD, $n=3564$ cells). Obviously, zyx-rsEGFP2 levels cannot anticipate negative values. The high standard deviation merely reflects the high variability of fusion protein expression levels that range from $10^{2}-10^{5} \mathrm{AU}$ as a consequence of heterologous overexpression. Compared to overexpression, FACS of endogenously tagged zyx-rsEGFP2 cells revealed a) that every cell is fluorescent, b) heterozygous cells exhibit an MFI of $903 \pm 575$ AU (SD, $n=11866$ cells) and c) homozygous 
cells display an MFI of $1080 \pm 590$ (SD, $n=11664$ cells). In contrast to endogenous zyxrsEGFP2 expression, protein levels are up to 100-fold increased upon transient transfection. Such an increase in protein levels is likely to induce changes of zyxin localization followed by a disturbance in cellular function (Nix et al., 2001). Interestingly, the MFI between both clones was similar although the heterozygous zyxin-rsEGFP2 carries only one tagged allele, while the homozygous knock-in cell line carries two ZYX-rsEGFP2 alleles. Intuitively, one might assume that fusion protein expression levels would differ by a factor of two between both clones. However, monoallelic gene expression from only one ZYX allele might occur while the second allele is silenced (Reinius and Sandberg, 2015).

The mean transfection efficiency of U2OS cells with a plasmid encoding HMGA1-rsEGFP2 was $35.5 \%$, while the mean MFI per cell was $62321 \pm 74841$ (SD, $n=12420$ cells) (Figure 39c). This observation is in agreement with the previous results that demonstrated highly heterogeneous fusion protein expression levels upon plasmid transfection. In fact, the HMGA1-rsEGFP2 level per single transfected cell can display a MFI value across four orders of magnitude $\left(10^{2}-10^{5} \mathrm{AU}\right)$, which is reflected by a standard deviation higher than the actual mean. While transient transfection results in $35.5 \%$ of fluorescent cells, the entire population of endogenously tagged HMGA1-rsEGFP2 cells is fluorescent. Heterozygous tagging of HMGA1 leads to a mean single cell expression level of $2530 \pm 1409$ AU (SD, $n=13157$ cells), while homozygous rsEGFP2 integration leads to HMGA1-rsEGFP2 expression levels of $9018 \pm 4158 \mathrm{AU}$ (SD, $\mathrm{n}=11626$ cells). Compared to overexpression, endogenous expression results in a 15-fold lower HMGA1-rsEGFP2 level representing the physiological expression level of the protein. Interestingly, the homozygous clone exhibits an about 4-fold higher fluorescence signal per cell compared to the heterozygous HMGA1-rsEGFP2 clone. This is consistent with the assumption that all HMGA1 alleles serve as templates for transcription resulting in a higher fusion protein level for homozygous knock-in cells.

In conclusion, transient plasmid transfection results in fusion protein levels that substantially vary over several orders of magnitude $\left(10^{2}-10^{5} \mathrm{AU}\right)$ in a single cell. In particular, an up to 100fold increase could be observed upon fusion protein overexpression compared to endogenous expression. Interestingly, phenotypic heterogeneity of fusion protein expression levels among genetically identical cells was observed for all endogenously tagged cell lines. This single cell heterogeneity or 'biological noise' arises from cellular differences in gene expression and cell cycle stage as well as from physical parameters such as cell size (Kaern et al., 2005). 


\subsubsection{Confocal microscopy of endogenously tagged and overexpressing cells}

Overexpression of fusion proteins is well known to cause a multitude of artefacts including protein aggregation and mislocalization as well as morphology changes of cells and organelles (Gibson et al., 2013; Rizzo, 2010). However, while FACS can be used to asses expression levels in thousands of cells in a sensitive manner easily, it does not provide any information about parameters such as protein localization. Instead, confocal fluorescence microscopy was used, because it allows the investigation of potential overexpression-induced artefacts in a spatial context on a single cell level. In particular, the fusion protein expression pattern was compared between plasmid-transfected and endogenously tagged U2OS cells. As a control, fixed U2OS were used in which the respective protein of interest was visualized using indirect immunofluorescence staining.

Immunostaining of fixed U2OS cells revealed the native localization of vimentin within the cytoplasm where the protein forms intermediate filaments (Figure 40a). HMGA1 is a nuclear protein widely distributed within this compartment, while zyxin is localized to discrete focal adhesion sites anchoring the respective cell to the extracellular matrix (ECM).

Overexpression of vimentin-rsEGFP2 induces an aberrant cellular morphology and densely bundled vimentin filaments in living U2OS cells (Figure 40b). This observation is in agreement with previous studies for other epithelial cells (Nishio et al., 2001) and highlights the importance of vimentin filaments for the maintenance of cellular integrity (Fuchs and Weber, 1994). Confocal imaging of HMGA1-rsEGFP2 overexpressing cells revealed artifacts such as protein aggregates within the nucleoplasm, cell morphology changes as well as fusion protein mislocalization in the cytoplasm. Apart from the observed artefacts, HMGA1 overexpression is also likely to have an impact on gene expression, because the protein is involved in chromatin architecture and regulation of basal transcription (Grosschedl et al., 1994). Microscopic inspection of zyxin-rsEGFP2 overexpressing cells revealed a high proportion of mislocalized nucleocytoplasmic fusion protein in most cells. Zyxin is a low abundance protein that is usually enriched in cell-cell and cell-substrate focal adhesion sites (Beckerle, 1997). However, overexpression leads to the accumulation of cytoplasmic zyxin, because it might not be incorporated into spatially restricted focal adhesion complexes anymore.

Confocal microscopy analysis of living endogenously tagged U2OS cells showed that the investigated proteins exhibited a native localization pattern: vimentin forms thin cytoskeletal intermediate filaments, HMGA1 is solely found in the nucleus and zyxin is localized to discrete focal adhesion sites (Figure 40c). Remarkably, fusion protein distribution in 
endogenously tagged cells resembled the pattern previously only observed in fixed and antibody-stained cells.

In summary, plasmid transfection results in artificially high fusion protein expression levels, which induce protein mislocalization and aggregation as well as changes in cellular morphology. On the contrary, endogenous integration of a fluorescent reporter at a genomic locus leads to physiological fusion protein levels and endogenously tagged cells are free of overexpression-induced artefacts. Further, this work shows that cellular artefacts are caused by non-physiological protein levels rather than by fusion of a fluorescent tag to a host protein. While transient transfection often leads to inconclusive and contradictory results, endogenous protein tagging will allow meaningful investigations of cellular dynamics in the future (Gibson et al., 2013).

a)
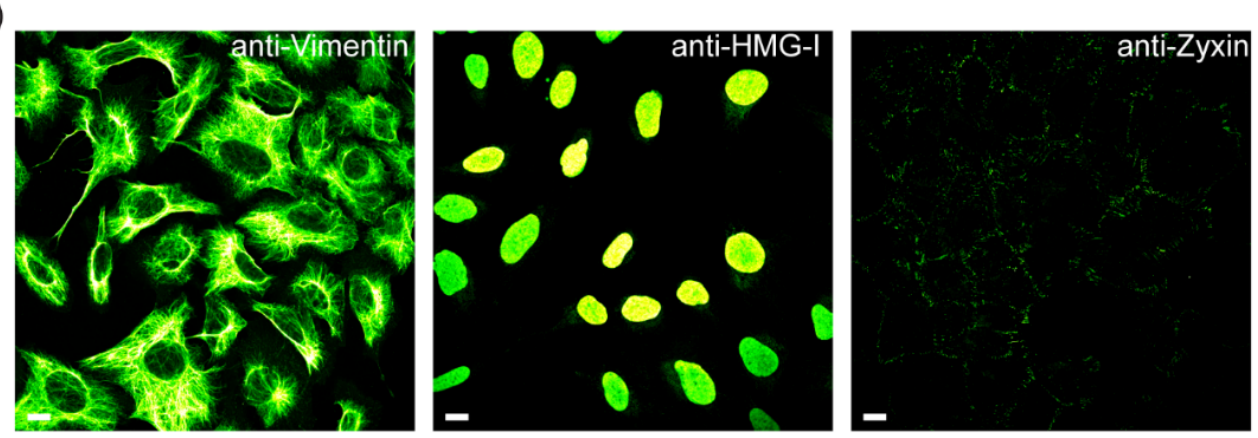

b)
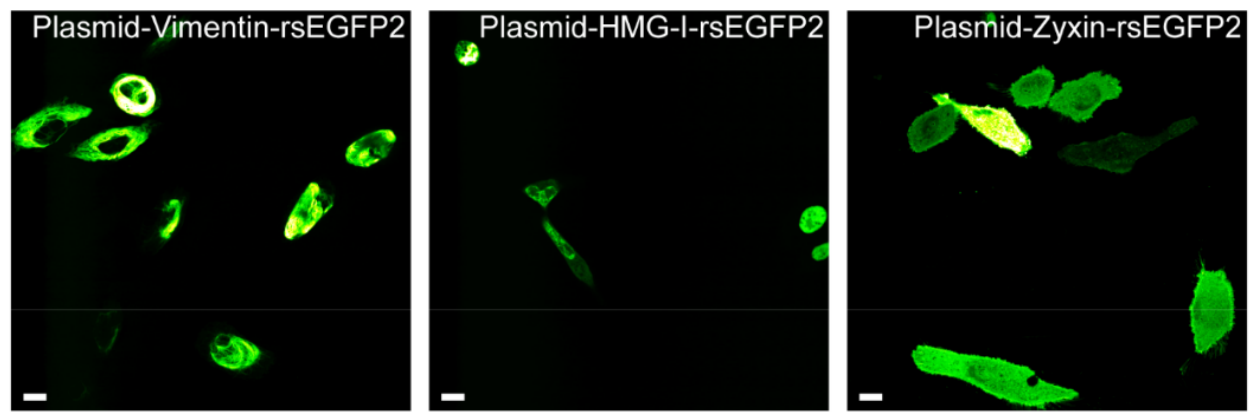

c)
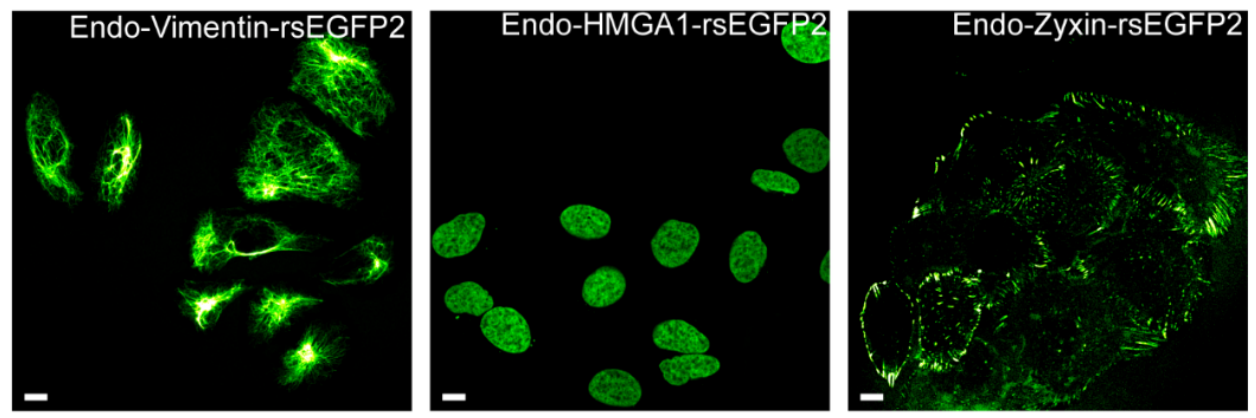

Figure 40: Fluorescence confocal imaging of U2OS cells. a) Fixed and immunostained U2OS cells show endogenous expression patterns of Vimentin, HMG-I and Zyxin. b) Living and transfected U2OS cells show heterogenous expression levels of Vimentin-, HMG-I and Zyxin-rsEGFP2 fusion proteins 
under the control of a cytomegalovirus (CMV) promoter. c) Living U2OS cells expressing vimentin-, HMG-I- and zyxin-rsEGFP2 fusion proteins from the endogenous locus show homogenous expression patterns comparable to immunostaining against the untagged protein in fixed cells. Scale bar: $10 \mu \mathrm{m}$. (Adapted from from Ratz et al., 2015).

\subsubsection{RESOLFT imaging of HMGA1-, VIM- and ZYX-rsEGFP2 cells}

RESOLFT super-resolution microscopy is particularly suited for investigating protein dynamics and distributions at the nanoscale in living cells (Grotjohann et al., 2012; Testa et al., 2012). While RESOLFT relied on transient overexpression so far, endogenous protein tagging provides the superior labelling strategy for live cell imaging, because it preserves native expression levels. A combination of the most suitable live cell super-resolution method with the most native labelling strategy is demonstrated in the following part.

First, RESOLFT microscopy was applied to image living cells expressing endogenously rsEGFP2-tagged vimentin (Figure 41a). Compared to diffraction-limited confocal microscopy, RESOLFT provides a superior resolution and nanoscale information can be retrieved. Without any image processing, we measured over stretches of thin vimentin-rsEGFP2 filaments a full width at half maximum of $\sim 40 \mathrm{~nm}$, based on Lorentzian fits to the data (Figure 41b). Subcellular dynamics could be recorded in a small field of view $\left(4.5 \times 4.5 \mu \mathrm{m}^{2}\right)$ on the seconds time scale (Figure 41c) as well as over the range of several minutes in a larger field of view of $12 \times 12 \mu \mathrm{m}^{2}$ (Figure 41d). 
a)

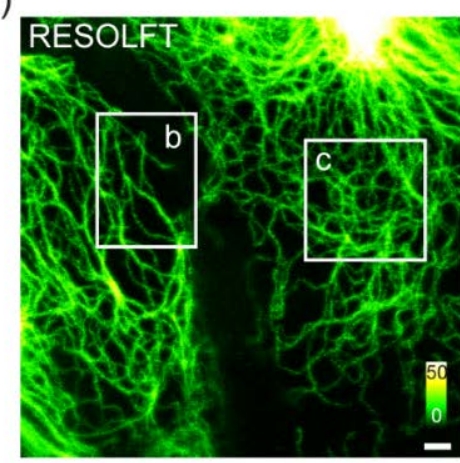

b)

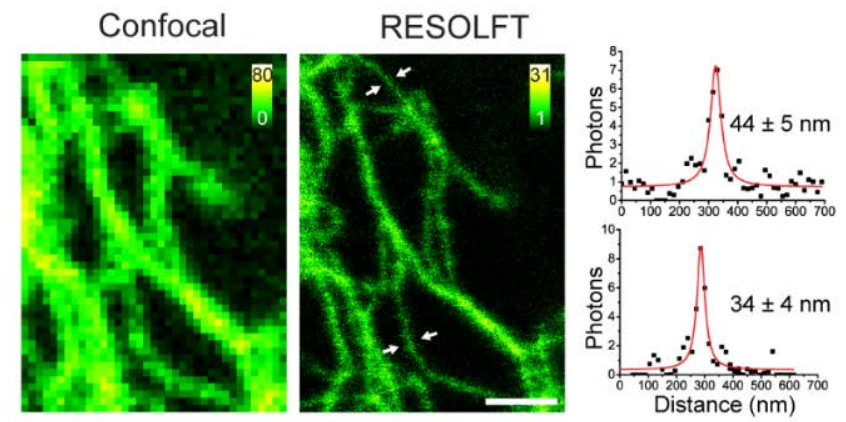

C)

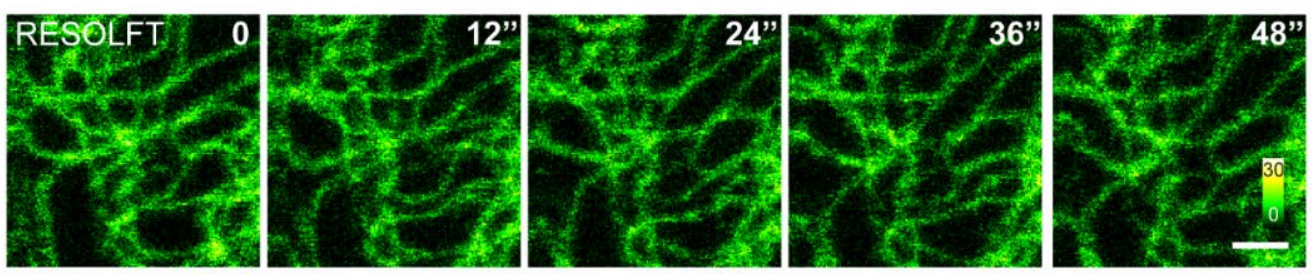

d)

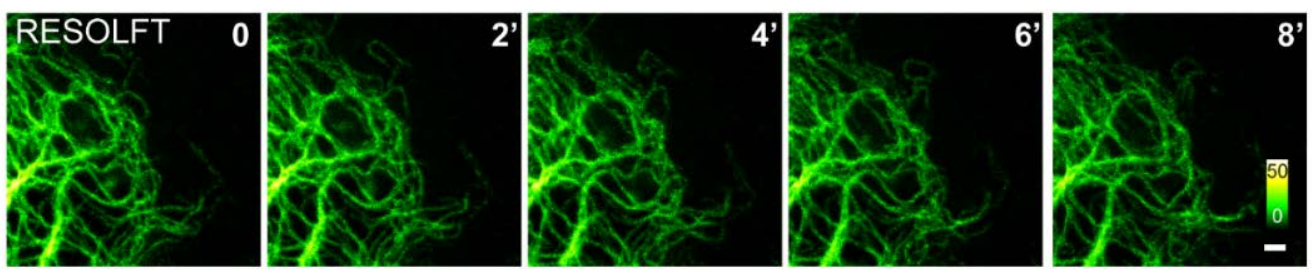

Figure 41: Live cell RESOLFT nanoscopy of vimentin-rSEGFP2 knock-in cells. a) Overview of two adjacent cells. b) Comparison of confocal and RESOLFT images. The images have been recorded in the area indicated in (a). Right: Intensity profiles across the filaments at the indicated sites (white arrows), each fitted with a Lorentzian function. The full width at half maximum (FWHM) values are given. (c) Repeated RESOLFT imaging in the area indicated in (a). Images were recorded every 12 seconds, as indicated. (d) Time-lapse RESOLFT imaging; images were recorded every 2 minutes, as indicated. (Adapted from Ratz et al., 2015).

Next, we analyzed living endogenously tagged HMGA1-rsEGFP2 cells using RESOLFT (Figure $42 \mathrm{a}, \mathrm{b}$ ). RESOLFT provides a superior resolution compared to confocal imaging exemplified by the fact that individual protein clusters could be distinguished. Further, dynamics of zyxin-rsEGFP2 knock-in cells could be captured at sub-diffraction resolution on a time-scale of several minutes (Figure 42c, d) and individual focal adhesion complexes distinguished from each other using RESOLFT. 
a)

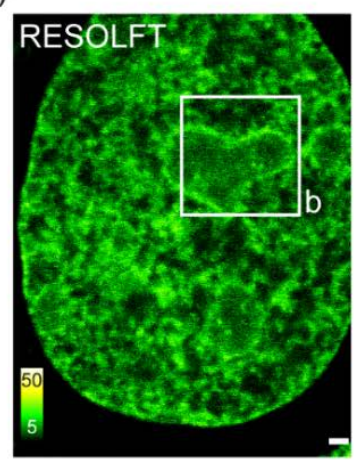

b)
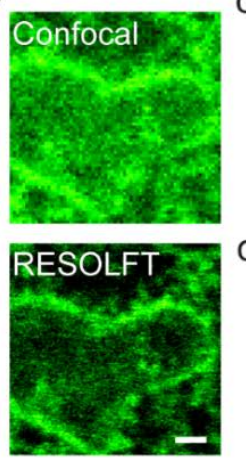

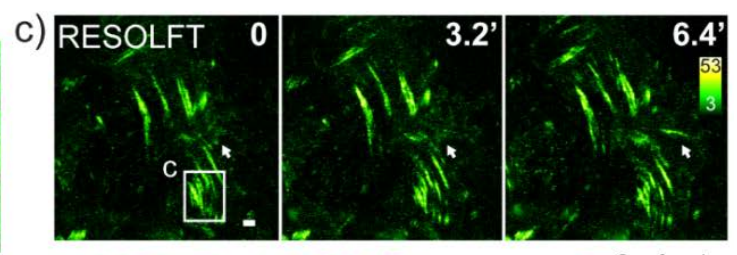

d) Confocal

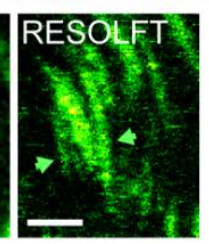

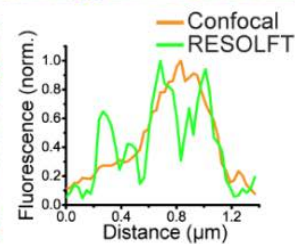

Figure 42: Live cell RESOLFT nanoscopy of HMG-I- and zyxin-rsEGFP2 knock-in cells. a) Overview RESOLFT image of a homozygous HMG-I-rsEGFP2 cell. b) Comparison of confocal and RESOLFT images. The images have been recorded in the area indicated in a). c) RESOLFT microscopy of homozygous zyxin-rsEGFP2 cells. Images were recorded every $3.2 \mathrm{~min}$, as indicated. (d) Comparison of confocal and RESOLFT images recorded in the area indicated in (c). Right: Intensity line profile across the area indicated in (d). All images display raw data. No deconvolution was applied. The RESOLFT image in (b) has been smoothed. Scale bars: 1 mm. (Adapted from Ratz et al., 2015).

In summary, this part presented the establishment of a general workflow for the generation of human cells expressing rsEGFP2 from a locus of interest using the CRISPR technology. The established methodologies include: 1) target selection, gRNA design, cloning and in vitro or in vivo functional testing; 2) donor plasmid generation using restriction enzyme based cloning or Gibson assembly; 3) clone isolation using single cell sorting and clonal expansion; 4) characterization of clonal lines via (out-out/junction) PCR, immunoblotting, Sanger sequencing, flow cytometry and confocal microscopy; 5) RESOLFT super-resolution imaging of endogenously tagged cells.

The established methodologies should be applicable to tag any human gene with a transgene of choice including fluorescent proteins, self-labelling enzymes or small protein tags. As outlined here, the entire protocol, from project planning to RESOLFT imaging, can be executed within 6 weeks. The entire workflow could be greatly accelerated by using a cell line that exhibits a faster growth rate than the here presented U2OS cells, because recovery of U2OS cells after single cell sorting takes about 2-3 weeks.

The workflow was applied to target three human genes (VIM, ZYX and HMGA1) for endogenous tagging with rSEGFP2 and both hetero- and homozygous knock-in cell lines were efficiently generated. DNA sequencing of on-target sites verified the site-specific transgene integration and absence of mutations at unwanted off-target sites indicates that the used gRNAs were highly sequence-specific. Moreover, endogenously tagged cells expressed the respective fusion protein at physiological levels and were free of overexpression-induced artefacts. Further, RESOLFT super-resolution microscopy was employed to visualize 
nanoscale protein dynamics in living knock-in cells over different timescales. In fact, live cell RESOLFT imaging on endogenously tagged cells is the method of choice to provide physiologically relevant information on protein dynamics at the highest attainable optical resolution.

\subsection{Localization, dynamics and abundance of mitochondrial prohibitins}

Prohibitins comprise a family of mitochondrial inner membrane proteins that is evolutionary conserved and ubiquitously expressed (Merkwirth and Langer, 2009). Prohibitin-1 (PHB1) and prohibitin-2 (PHB2) were shown to form large ring assemblies in the inner mitochondrial membrane of yeast, while various subcellular localizations have been proposed for PHB1 and PHB2 in mammalian cells (Merkwirth and Langer, 2009; Artal-Sanz and Tavernarakis, 2009). However, lack of antibodies and overexpression-induced artefacts hampered the detailed study of mammalian prohibitin localization so far. Further, dynamics and abundance of PHB1 and PHB2 are unknown. Here, we show that PHB1 or PHB2 overexpression induces two different aberrant mitochondrial morphologies and that these artefacts can be avoided upon CRISPR-mediated endogenous tagging of PHB1 and PHB2. The endogenously tagged prohibitin cell lines were used to demonstrate the submitochondrial prohibitin localization via immunogold EM and cluster formation of PHB1 and PHB2 using STED microscopy. Further, prohibitin dynamics were analyzed using FRAP measurements and the abundance of each prohibitin molecule per cell was quantified using western blotting. Finally, this information was integrated to estimate the amount of prohibitin complexes per mitochondrial cristae.

\subsubsection{Overexpression analysis of PHBs}

Transient overexpression of fusion proteins is widely used to study e.g. the subcellular localization of the fusion protein. However, overexpression of PHB1 or PHB2 fused to the reversibly switchable fluorescent protein Dreiklang (DK) caused an aberrant mitochondrial morphology in the majority of transfected cells (Figure 43). 
a)
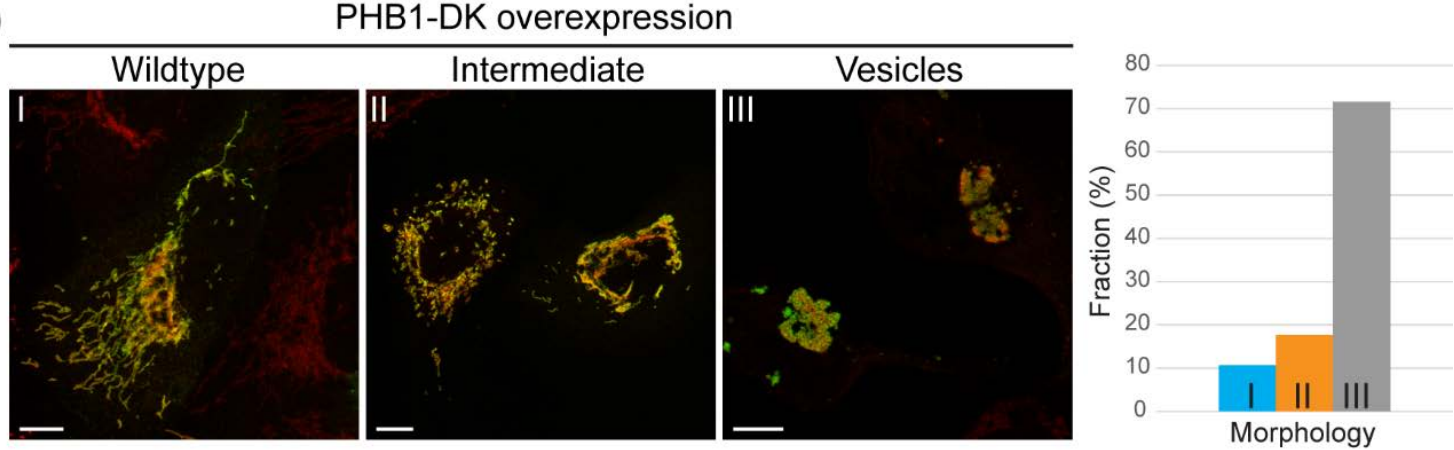

b)

PHB2-DK overexpression
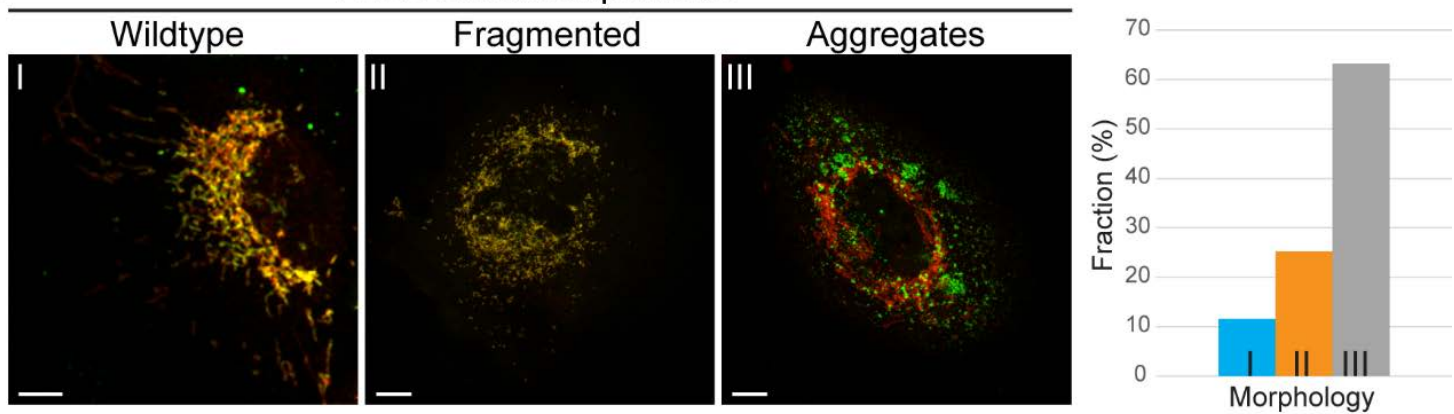

Figure 43: Overexpression of PHB1-DK and PHB2-DK in U2OS cells. a) PHB1-DK overexpression induces different mitochondrial morphologies: wildtype (I), intermediate (II) and vesicular (III). b) PHB2DK overexpression induces another set of phenotypes: wildtype mitochondria (I), fragmented mitochondria (II) and cytoplasmic protein aggregates (III). Scale bar: $10 \mu \mathrm{m}$.

Only 11\% of the PHB1-DK expressing cells displayed a wildtype mitochondrial morphology, while $72 \%$ of the cells displayed highly aberrant mitochondria that formed perinuclear clusters of enlarged mitochondria (Figure 43a). An intermediate phenotype displaying both partially clustered and retracted mitochondrial tubules was observed in $17 \%$ of PHB1-DK positive cells.

Interestingly, PHB2-DK overexpression induced a phenotype different from PHB1-DK overexpression. Here, only $12 \%$ of the PHB2-DK positive cells exhibited a wildtype mitochondrial morphology and $63 \%$ of the cells showed an accumulation of fluorescent PHB2-DK aggregates (Figure 43b). The majority of those aggregates was cytoplasmic and did not co-localize with mitochondria that were specifically stained using an anti-TOM20 antibody. This observation indicates an inhibited mitochondrial import of PHB2-DK at high expression rates. Further, 25\% of the PHB2-DK overexpressing cells displayed fragmented mitochondria in which co-localization of PHB2-DK and TOM20 still occurred.

In conclusion, overexpression of PHB1-DK mainly results in perinuclear clustering of enlarged mitochondria, while PHB2-DK overexpression causes mitochondrial fragmentation or 
accumulation of cytoplasmic protein aggregates. Although the underlying molecular mechanisms remain to be investigated, it can be concluded that artificially high prohibitin levels disrupt the tubular structure of mitochondrial.

\subsubsection{Endogenous tagging of PHBs}

As transient transfection of both PHB1-DK and PHB2-DK mainly resulted in aberrant mitochondrial morphologies, we decided to use CRISPR-mediated endogenous tagging for labelling of both proteins. Prohibitins are targeted to mitochondria by an unconventional noncleavable targeting sequences at their N-terminal end (Tatsuta et al., 2005). Positioning of an N-terminal tag caused accumulation of prohibitin in the cytoplasm of baby hamster kidney cells, while C-terminal c-Myc tagging lead to mitochondrial accumulation (Ikonen et al., 1995). Similary, C-terminal GFP tagging was successfully done for ectopically expressed PHB1 and PHB2 in HeLa cells (Kasashima et al., 2006) and for PHB2 in mouse cells (Merkwirth et al., 2008; Kowno et al., 2014). In order to accomplish C-terminal tagging of PHB1 and PHB2 also on an endogenous level, two gRNAs were designed that target the stop codon region of each respective prohibitin (Figure 44 ).

a) PHB1 stop codon region

gRNA2

gRNA1

5' - . . TCCGTGCTCCTCCAGCTGCCCCAGTGAGGGCCACCCTGCCTGCACCTCCGCGGGCTGACTGGGCCACAGCCCCGATGAT . .-3'

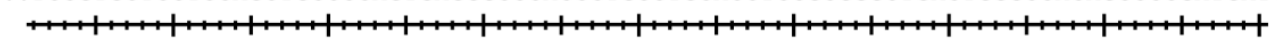
3' - . . AGGCACGAGGAGGTCGACGGGGTCACTCCCGGGTGGGACGGACGTGGAGGCGCCCGACTGACCCGGTGTCGGGGCTACTA ...-5' \begin{tabular}{lllllllllll}
\hline \multicolumn{7}{|c|}{ Exon 6} \\
\hline$S$ & $\vee$ & $L$ & $L$ & $Q$ & L & $P$ & $Q$ & \\
\hline
\end{tabular}

b) PHB2 stop codon region

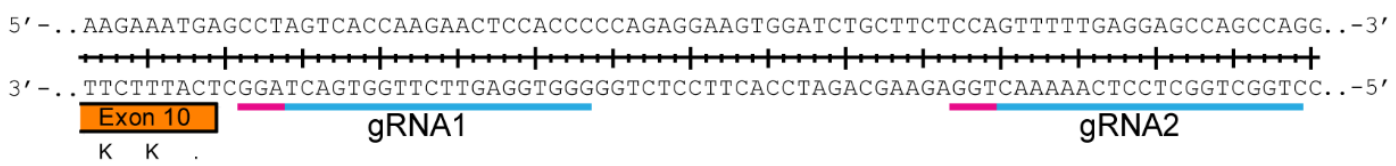

Figure 44: gRNA design for C-terminal tagging of PHB1 and PHB2. Two gRNAs were designed for targeting the stop codon region of human PHB1 (a) or PHB2 (b), respectively. Light blue: gRNA binding site; Magenta, PAM site.

Using the CRISPR Design Tool (Hsu et al., 2013), gRNAs that mediate a Cas9-induced DSB within the non-coding 3'-UTR of each gene and with the lowest amount of associated offtarget sites were chosen. Each gRNA was inserted into pX330 using the oligo cloning protocol described previously (section 3.2.2).

Generation of donor plasmids for the integration of the fluorescent protein Dreiklang (DK) was done using Gibson assembly (Gibson et al., 2009). Therefore, the respective left and right 
homology arms were amplified from U2OS genomic DNA using the primers listed in Table 5. The coding sequence of DK was amplified from a plasmid. Gibson assembly resulted in two donor plasmids for PHB1 and PHB2, respectively, bearing DK flanking homology regions between 832 and 969 bp (Figure 45).

a)

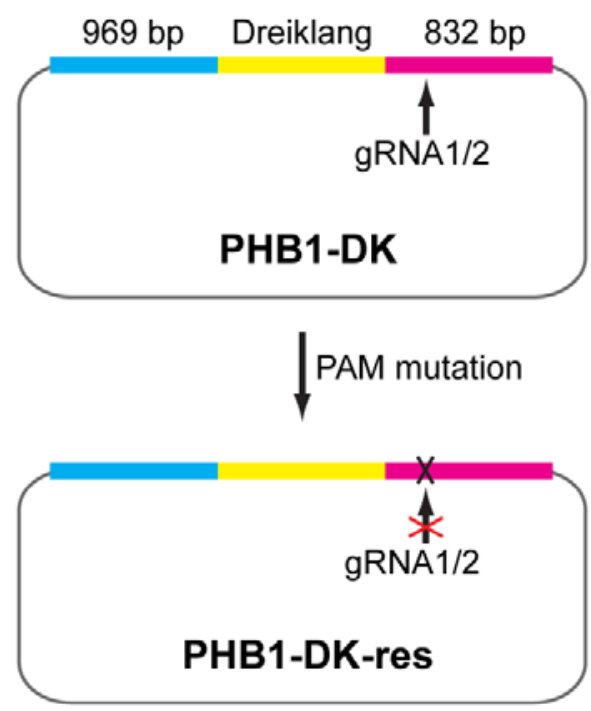

b)

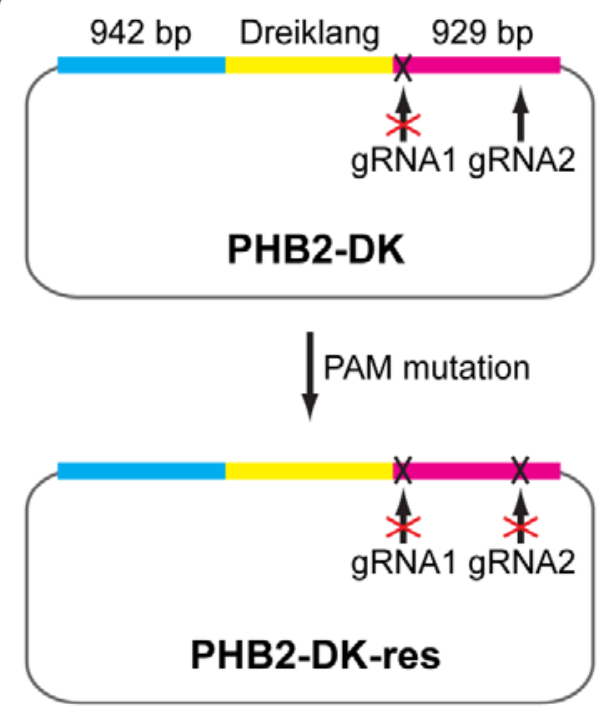

Figure 45: Generation of Cas9-resistant donor plasmids for C-terminal tagging of PHB1 and PHB2. Each donor plasmid was generated using Gibson assembly and comprises a left (light blue) and right homology arm (magenta) with a length of about 800-1000 bp. a) The PHB1-DK donor plasmid contained PAM sites for both gRNA1 and gRNA2 within the sequence TGGG. Site-directed mutagenesis was used to change this sequence to TAAG thereby making the plasmid nucleaseresistant. b) The initially constructed PHB2-DK donor plasmid was designed such that the PAM required for gRNA1 targeting was mutated. The PAM sequence 'AGG' was mutated to 'ATT' thereby making the donor plasmid resistant to gRNA2 targeting.

Importantly, the previous experience with endogenous tagging showed that 'nucleaseresistant' donor plasmids can be used to avoid secondary Cas9-induced mutagenesis after transgene integration (section 1.4.2). Further, it was suggested that Cas9-resistant donor plasmids would lead to increased recombination efficiencies as they are not degraded by Cas9 in cells (Ran et al., 2013b). Because Cas9 cleavage requires the presence of a PAM site with the sequence NGG, each donor plasmid was modified minimally by changing the respective PAM. In particular, PHB1-gRNA1 relies on the presence of GGG for Cas9-induced DNA cleavage, while PHB1-gRNA2 requires the sequence TGG as adjacent PAM in the target site (Figure 45a). Because both PAM sites overlap within the sequence TGGG, a single mutagenesis step that changed this sequence to TAAG was sufficient to make the PHB1-DK donor resistant towards both gRNAs (Figure 34a). Moreover, primer design for the PHB2-DK 
donor plasmid was done such that the PAM sequence 'AGG' required for gRNA1 was mutated to 'ATG' already within the initial donor plasmid. Site-directed mutagenesis was used to change the second PAM site 'AGG' to 'ATT' resulting in a gRNA2-resistant PHB2-DK donor plasmid (Figure 45b). Importantly, the new donor plasmids 'PHB1-DK-res' and 'PHB2DK-res' do not contain any other modifications than the mentioned PAM sequence mutations rendering them essentially Cas9-resistant.

Each gRNA specific for either PHB1 or PHB2 was co-transfected together with the respective Cas9-susceptible donor plasmid or the Cas9-resistant version of the donor plasmid. Seven days after co-transfection, U2OS cells were analyzed via FACS (Figure 46). In absence of any nuclease encoding plasmid, the fraction of DK+ cells is about $0.6 \%$ for each donor plasmid (Figure 46a). Co-transfection of PHB1-gRNA1 resulted in $6.6 \%$ fluorescent cells using the PHB1-DK donor with intact PAM site and increased to $12.6 \%$ using the Cas9resistant PHB1-DK donor (Figure 46b). Further, co-transfection of PHB1-gRNA2 resulted in 2.1\% DK-positive cells when the nuclease-susceptible donor was used, while the fraction of fluorescent cells increased to $4.3 \%$ upon usage of the nuclease-resistant donor plasmid (Figure 46c). Similarly, a more than 2 -fold increase in fluorescent cells from $8.2 \%$ to $17.7 \%$ was observed when PHB2-gRNA1 was co-transfected with Cas9-resistant PHB2-DK donor plasmid (Figure 46d). Finally, co-transfection of PHB2-gRNA2 with the Cas9-susceptible donor plasmid resulted in $8.6 \%$ DK-positive cells, whereas this value increased to $10 \%$ when the nuclease-resistant donor was co-transfected (Figure 46e). In conclusion, homologous recombination efficiencies could be significantly increase for most of the analyzed gRNAs when a nuclease-resistant donor was used (Figure 46f). Because Cas9 does not degrade a donor plasmid that lacks a PAM site, a higher plasmid amount is available for recombination. 
a)

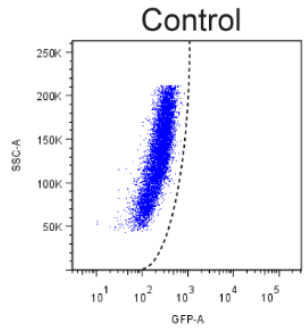

b)

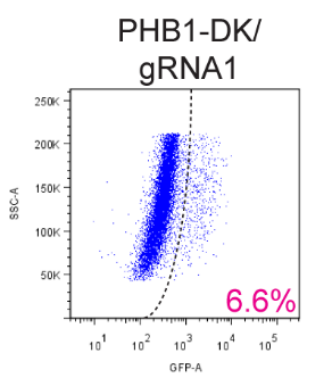

c)

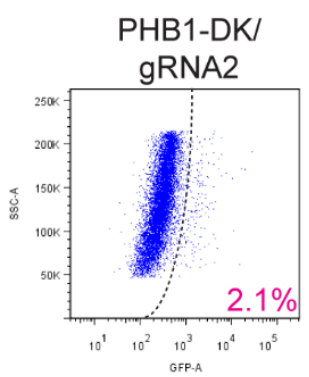

d)
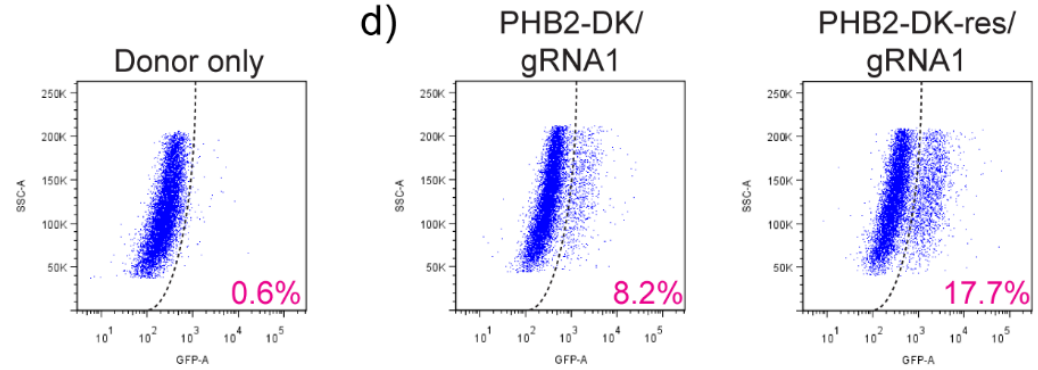

e)
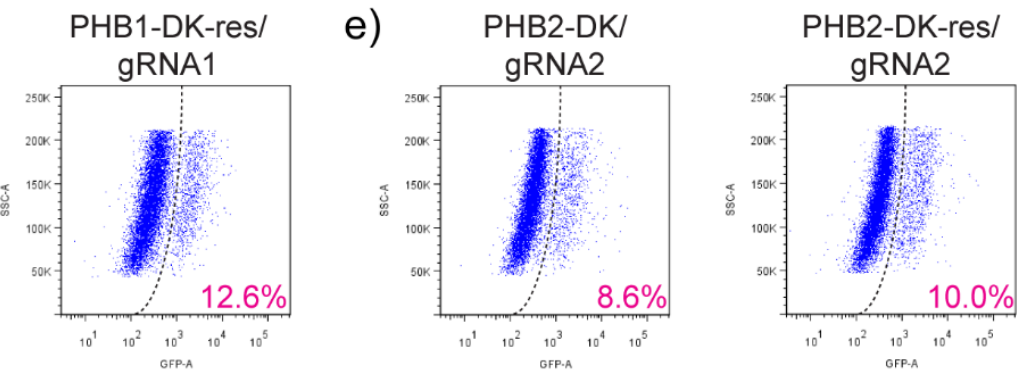

f)
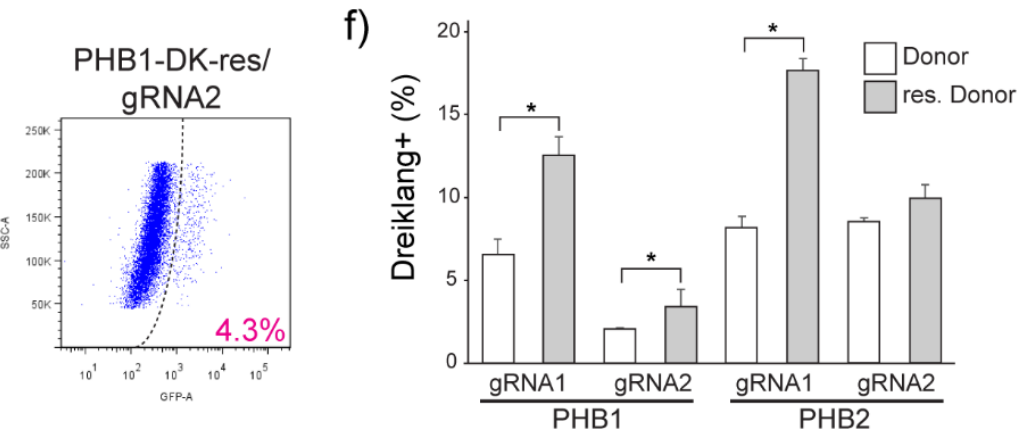

Figure 46: FACS analysis of U2OS cells after co-transfection with PHB1 and PHB2 targeting nuclease and donor plasmids. a) Wildtype and donor plasmid only transfected U2OS cells were used as control. b, c) Co-transfection of PHB1-gRNA1 (b) or PHB1-gRNA2 (c) with Cas9-susceptible PHB1DK or Cas9-resistant PHB1-DK-res donor. d, e) Co-transfection of PHB2-gRNA1 (d) or PHB2-gRNA2 (e) with Cas9-susceptible PHB2-DK or Cas9-resistant PHB2-DK-res donor. The mean fraction of DK+ cells of three independent experiments is shown. f) Use of Cas9-resistant donor plasmids increases targeting efficiency significantly for most nuclease/donor plasmid pairs.

Single cell sorting after co-transfection was done for each combination of gRNA with the respective nuclease-resistant donor plasmid. Per nuclease/donor plasmid combination, six 96-well plates were sorted. Tagging of PHB1 using gRNA1/PHB1-DK-mut resulted in 62 wells $(10.8 \%)$ that contained a colony of cells. Use of gRNA2/PHB1-DK-mut resulted in 40 colony containing wells (6.9\%). For PHB2 tagging, use of gRNA1/PHB2-DK-mut resulted in 72 positive wells (12.5\%) and using gRNA2/PHB2-DK-mut yielded 63 wells (10.9\%) containing cell colonies. Those cells were split and replicated for further analysis. Inspection of the cells via fluorescence microscopy resulted in 20 (gRNA1/PHB1-DK-mut) and 29 (gRNA2/PHB1DK-mut) successfully tagged PHB1-Dreiklang clones. Further, 31 (gRNA1/PHB2-DK-mut) 
and 43 (gRNA2/PHB2-DK-mut) targeted PHB2-Dreiklang clones were obtained. Genotyping of these cells was done using in-out PCR for PHB1-DK and out-out PCR for PHB2-DK clones (Figure 47).

a)

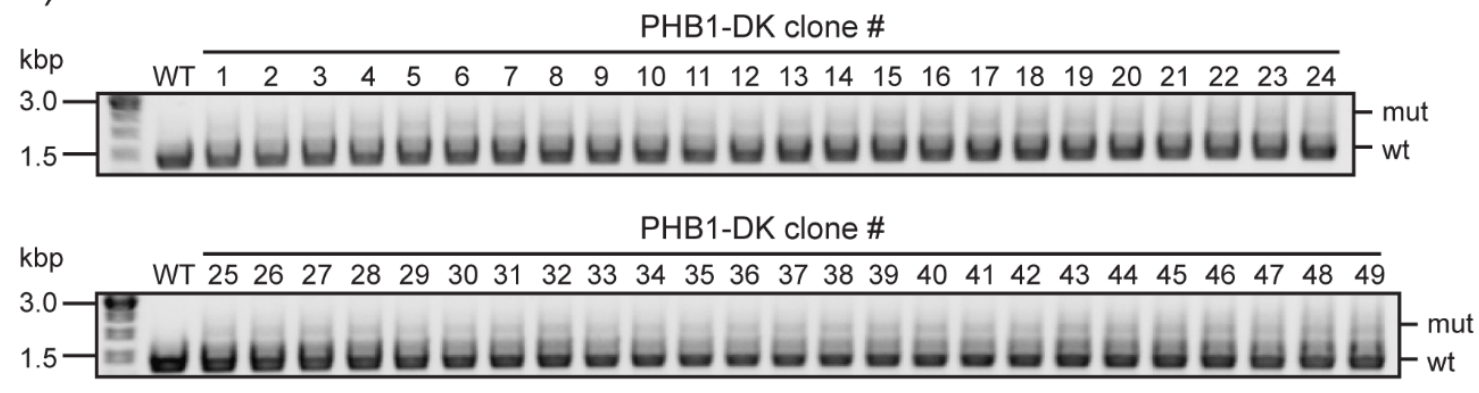

b)

PHB2-DK clone \#

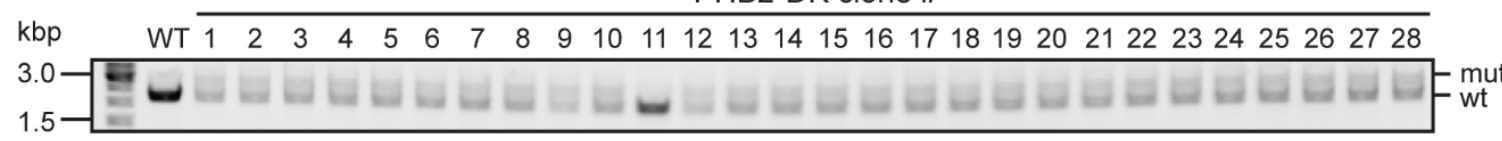

PHB2-DK clone \#
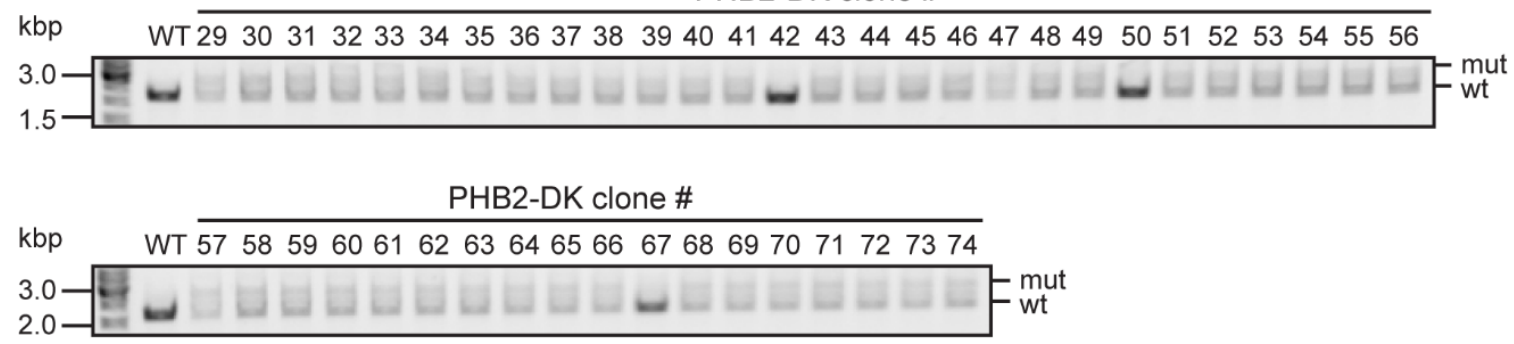

Figure 47: Genotyping of PHB1-DK and PHB2-DK knock-in cells. a) Out-in PCR analysis of PHB1DK clones generated with PHB1-gRNA1 (clones 1-20) or PHB1-gRNA2 (clones 21-49), respectively b) Out-out PCR analysis of PHB2-DK clones generated with PHB2-gRNA1 (clones 1-31) or PHB2-gRNA2 (clones 32-74), respectively.

All 49 generated PHB1-DK clones were heterozygous for the FP knock-in. Further, except for four clones showing a wildtype PCR band only, all 70 PHB2-DK clones were heterozygous as well. In conclusion, while homologous recombination occurred at high frequencies and several knock-in clones for both PHB1-DK and PHB2-DK were obtained, no homozygous knock-in was achieved. While only homozygous knock-in cells would allow counting of local prohibitin copy numbers in single complexes, heterozygous cells are a great model system to investigate the unknown localization, dynamics and global amounts of prohibitins.

As PHB1-gRNA2 and PHB2-gRNA2 were initially predicted to have the lowest amount of offtarget cleavage sites, four clones from each of the respective PHB1-DK or PHB2-DK pools 
were picked for protein expression analysis (Figure 48). Compared to PHB1 expression levels in wildtype U2OS cells, total PHB1 protein levels were elevated between 1.8- and 12.2-fold. Analysis of total PHB2 levels in PHB2-DK cells revealed a slight reduction in total PHB2 concentrations that were between 0.61-0.99-fold the concentrations found in wildtype cells. It can be concluded that genomic tagging does not necessarily result in physiological expression levels and that multiple knock-in clones should be analyzed before selecting a clone for further analysis.

a)

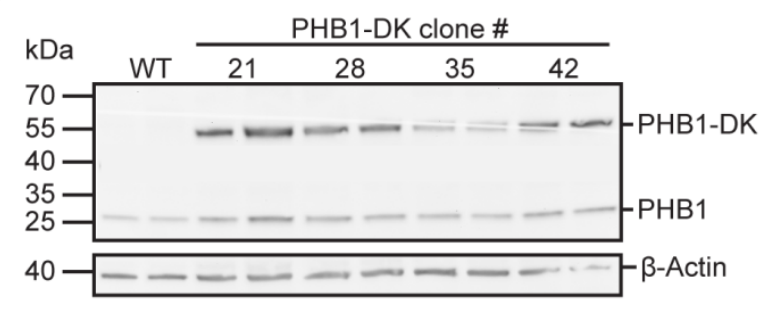

b)

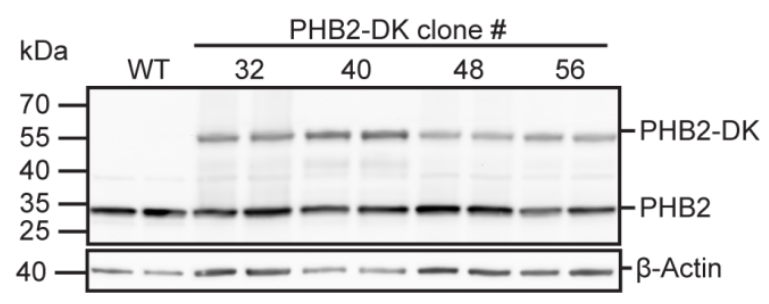

c)

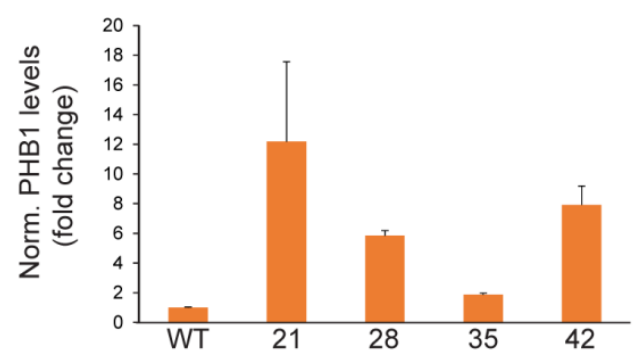

d)

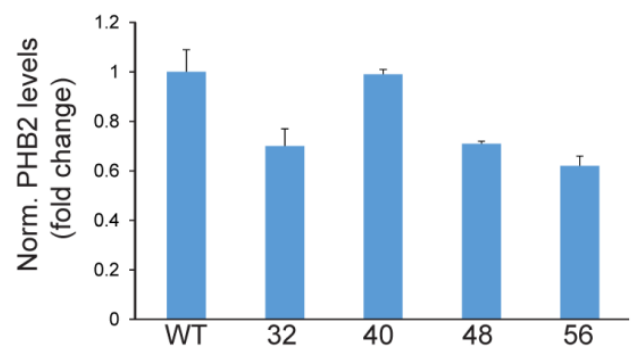

Figure 48: Protein expression level analysis in PHB1-DK and PHB2-DK clones. a, b) Extracts of four PHB1-DK cell lines (a) or four PHB2-DK cell lines (b) were analyzed via immunoblotting using antibodies against PHB1 (a) or PHB2 (b), respectively. Wildtype (WT) extract was loaded as a reference. Actin was detected as internal loading control. c, d) Band intensities were quantified, PHB1 (c) or PHB2 (d) expression levels corrected for variations in loaded amounts and normalized to the PHB1 (c) or PHB2 (d) expression level in wildtype (WT) cells.

For further experiments, PHB1-DK clone 35 and PHB2-DK clone 40 were selected as those cell lines showed most similar expression levels of the respective protein when compared to wildtype cells. For simplicity these clones will be referred to as PHB1-DK and PHB2-DK in the following parts.

As overexpression of PHB1-DK and PHB2-DK induced aberrant mitochondrial morphologies, endogenously tagged PHB1-DK and PHB2-DK cells were first analyzed using confocal microscopy (Figure 49). Living genome-edited U2OS cells were first incubated with MitoTracker ${ }^{\circledR}$ Red FM to selectively highlight mitochondria and subsequent confocal imaging showed that both prohibitins localized to mitochondria. Further, mitochondria of endogenously 
tagged PHB1-DK and PHB2-DK cells exhibited wildtype morphology indicating that imbalanced protein levels, but not fluorophore tagging, induced the previously observed aberrant mitochondrial phenotypes.

a)
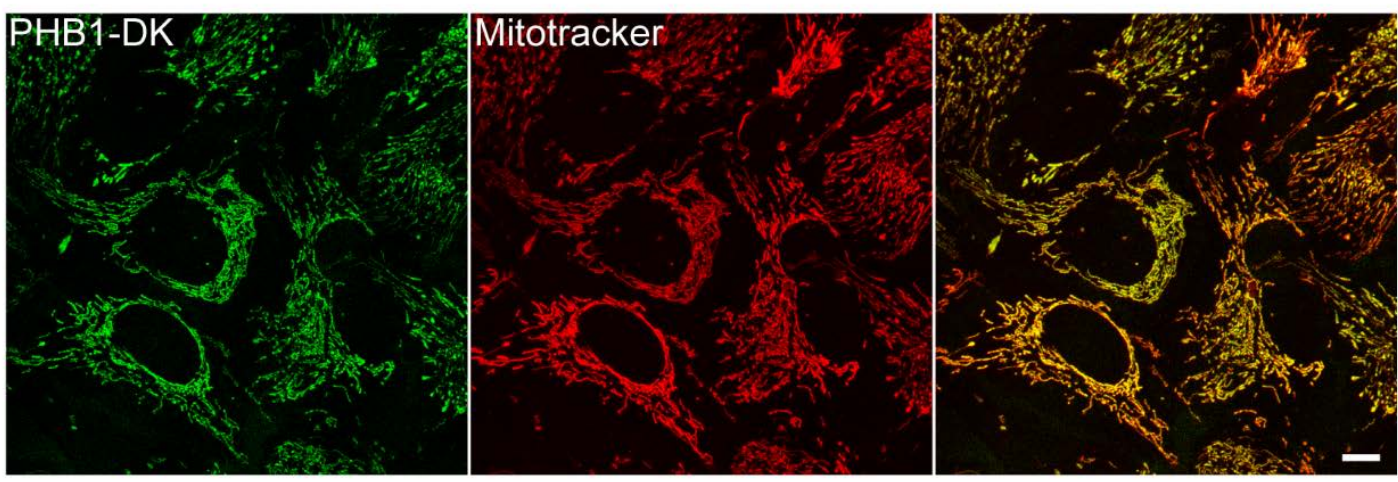

b)
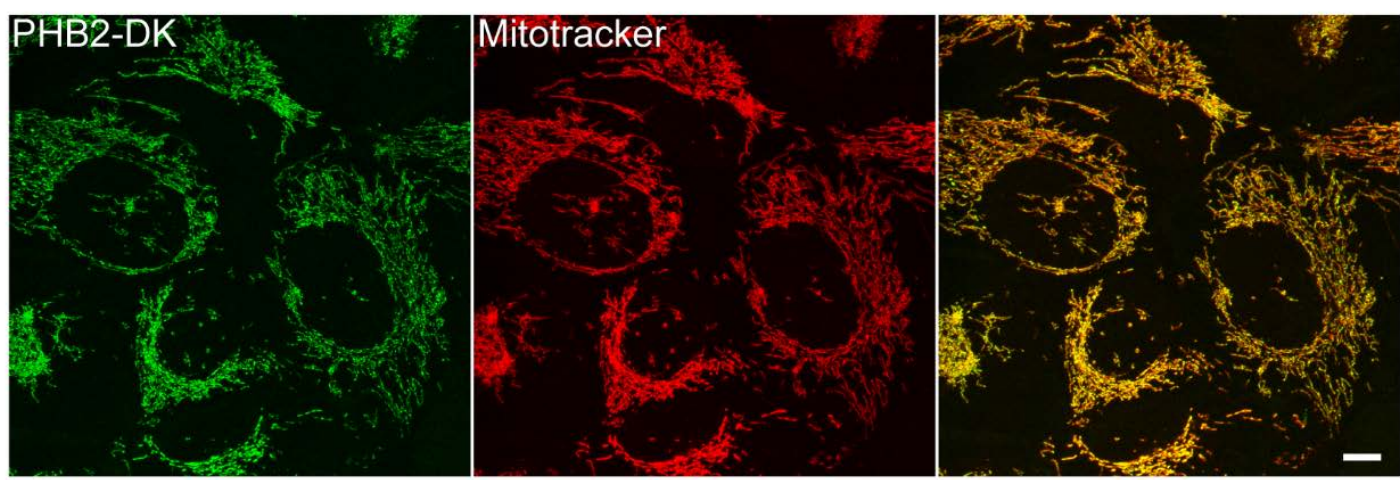

Figure 49: Confocal microscopy of PHB1-DK and PHB2-DK knock-in cells. Mitochondrial morphology was analyzed in living PHB1-DK (a) and PHB2-DK (b) cells. Mitochondria were marked using Mitotracker Deep Red FM. Scale bar: $10 \mu \mathrm{m}$

Next, Sanger sequencing was used to analyze potential on-target site modifications in PHB1DK cell lines (Figure 50a, b). No mutations were found at the PHB1-gRNA2 site of the DKtagged allele. This was expected as the introduced PAM site mutations in the PHB1-DK donor plasmid inhibit further Cas9-induced cleavages. Also the untagged PHB1 allele was not containing any mutations. This was surprising, because untagged alleles were constantly found to contain small indel mutations in previous DNA sequencing studies of gRNA on-target sites (section 1.3.2). However, this indicates that indeed not all PHB1 gene copies are accessible for site-specific gene editing as suggested previously. While four copies of the PHB1 gene exist in U2OS cells (Halling-Brown et al., 2012) and one is definitely containing the transgene, it is not trivial to determine the actual amount of (un-)tagged alleles as this requires advanced methods such as digital PCR (Hindson et al., 2011). 
Sanger sequencing was also applied to investigate the gRNA2 target site in PHB2-DK cells. Except for the intentionally introduced PAM site mutations, no DNA sequence modifications were introduced at the tagged allele (Figure 50c, d). This was expected and demonstrates that Cas9 cleavage could be efficiently blocked via PAM mutations. However, three different DNA sequences were found to represent untagged PHB2 gene copies. The first sequence was identical to the wildtype reference, which indicates that it is not accessible for Cas9mediated modifications. The second sequence contained a $14 \mathrm{bp}$ deletion and the third sequence lacked 20 nucleotides. Compared to the previously observed mutations that mainly affected the gRNA seed region (section 1.3.2), these mutations are larger and result in nearly complete removal of the gRNA target site. The observation of two different mutational patterns at the same target site confirms that NHEJ-mediated repair of DSBs is an error prone process and its outcome is difficult to predict (Caldrick et al., 2013). Further, U2OS cells harbor five PHB2 gene copies (Halling-Brown et al., 2012). While three untagged alleles were found and at least one tagged allele exists, it cannot be said whether the remaining allele contains the transgene or not. To determine the exact ratio of tagged vs. untagged alleles, copy number variation analysis would be required.

a)

$\begin{array}{ll}\frac{\text { PHB1-Exon 6 }}{\text { Untagged }} & \text { AGCTGCCCCAGTGAGGGCCCACCCTGCCTGCACCTCCGCGGGCTGACTGG } \\ \text { PHB1-DK } & \text { AGCTGCCCCAGTGAGGGCCCACCCTGCCTGCACCTCCGCGGGCTGACTGG }\end{array}$

b) $\begin{array}{llc} & \frac{D K}{\text { Tagged }} & \text { ACAAGTAAGGTACCGGGCCCACCCTGCCTGCACCTCCGCGGGCTGACTGG } \\ \text { PHB1-DK } & \text { ACAAGTAAGGTACCGGGCCCACCCTGCCTGCACCTCCGCGGGCTGACTAA }\end{array}$

c) PHB2-Exon 10 PAM \ PHB2-gRNA2 Untagged AGGGTAAGAATGAN ${ }_{37}$ ATCTGCTTCTGGAGTTTTTGAGAGCCAGCCAGGGG PHB2-DK \#1 AGGGTAAGAAATGAN ${ }_{37}$ ATCTGCTTCTGGAGTTTTTGAGGAGCCAGCCAGGGG PHB2-DK \#2 AGGGTAAGAAATGAN 37 ATCTGCTTCTGGAGTT-------------CAGGGG PHB2-DK \#3 AGGGTAAGAAATGAN 37 ATCTGCTTCTGGAGTT-

d)

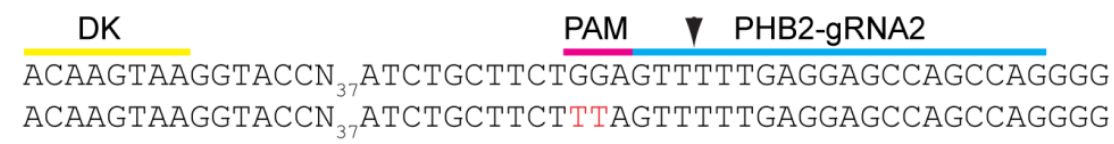

Figure 50: On-target sequencing of PHB1-DK and PHB2-DK cell lines. $a, b)$ Sequencing of the selected heterozygous PHB1-DK clone revealed that the untagged PHB1 allele is unmodified (a) and that the tagged PHB1-DK allele contains the expected mutations previously introduced into the donor plasmid (b). c) Sequencing of the selected heterozygous PHB2-DK clone revealed that the untagged PHB2 alleles contain the unmodified DNA sequence (1), a 14 bp deletion (2) or a 20 bp deletion (3). d) 
The tagged PHB2-DK allele contains the expected PAM site mutations previously introduced into the donor plasmid.

For each nuclease, the top three predicted off-target sites (Table 3) were sequenced using TOPO-cloned PCR products. Multiple bacterial colonies were screened via colony PCR after TOPO cloning and 15-20 plasmids containing the expected insert were sent for sequencing.

Table 3: Predicted off-target sites for PHB1-gRNA2 and PHB2-gRNA2. The top three off-target (OT) sites as predicted using the CRISPR Design Tool are listed. Number of nucleotide mismatches and their position with respect to the on-target site as well as the locus and gene name for each OT are given.

\begin{tabular}{cccccc}
\hline gRNA & OT & OT sequence (5' to 3') & Mismatches & Locus & Gene \\
\hline \multirow{2}{*}{ PHB1- } & 1 & CTGCACCTCCGCAGGCTGACTGG & $1[13]$ & chr12: -51518326 & $\mathrm{nc}$ \\
gRNA2 & 2 & CTCCACTTCCGCGGGCTGACCGG & $2[3: 7]$ & chr1:-224044240 & $\mathrm{nc}$ \\
& 3 & CTGCACCGCCGTGGGCTGACTGG & $2[8: 12]$ & chr16:+52969561 & $\mathrm{nc}$ \\
\hline \multirow{2}{*}{ PHB2- } & 1 & CTTGCTGGCTCTTCAAAAACAAG & $2[3: 12]$ & chr4:+37660648 & $\mathrm{nc}$ \\
gRNA2 & 2 & CAGGCTGGCTCCTCCAAAACAAG & $2[2: 15]$ & chr9:-136215687 & RPL7A \\
& 3 & CTGCCTCACTCCTCAAAAACGGG & $3[4: 7: 8]$ & chr22:-44562291 & $\mathrm{nc}$ \\
\hline
\end{tabular}

PHB1-gRNA2 was predicted to target non-coding DNA sequences found on chromosomes 12, 1 and 16. Those nucleotide sequences differed in only 1-2 bp compared to the on-target cleavage site and furthermore contained NGG PAM sequences. Therefore, it was surprising that none of the predicted off-target sites showed any unwanted mutations in PHB1-DK cells (Figure 51a). This confirms that not only the number, but also the position as well as the spacing of mismatches between gRNA and potential off-target site have an influence on Cas9 activity (Ran et al., 2013b; Hsu et al., 2013).

Next to the on-target, PHB2-gRNA2 could potentially target three similar sequences found on chromosomes 4, 9 and 22. One off-target site lies within intron 1 of the human RPL7A gene encoding ribosomal protein L7a, an essential structural constituent of the $60 \mathrm{~S}$ ribosomal subunit. However, DNA sequencing of this region as well as the other two regions in PHB2DK cells revealed that all potential off-target sites were not targeted for mutagenesis by Cas9 (Figure 51b). Again, this indicates that the CRISPR Design Tool can be used for the generation of efficient and highly specific gRNAs. 
a)

WT

PHB1-DK

GAGGGCCCACCCTGCCTGCACCTCCGCAGGCTGACTGGGCCACAGCCCCA

GAGGGCCCACCCTGCCTGCACCTCCGCAGGCTGACTGGGCCACAGCCCCA

OT2: PHB1-gRNA2 \ PAM

WT GAGGGCCCACACGGCCTCCACTTCCGCGGGCTGACCGGGCCACAGCCCCG

PHB1-DK GAGGGCCCACACGGCCTCCACTTCCGCGGGCTGACCGGGCCACAGCCCCG

GAGGGCCCATCCTGCCTGCACCGCCGTGGGCTGACTGGGGCACAGCTCCG

PHB1-DK GAGGGCCCATCCTGCCTGCACCGCCGTGGGCTGACTGGGGCACAGCTCCG

b)

WT

PHB2-DK

TGGGATCCCTACAGCCTTGCTGGCTCTTCAAAAACAAGCCCATCACTTCA

TGGGATCCCTACAGCCTTGCTGGCTCTTCAAAAACAAGCCCATCACTTCA

WT

GTCGGGGGTAGGGCTCAGGCTGGCTCCTCCAAAACAAGGGACGCTGACAG

PHB2-DK GTCGGGGGTAGGGCTCAGGCTGGCTCCTCCAAAACAAGGGACGCTGACAG

WT

OT3: PHB2-gRNA2 \ PAM

CCTCTGGACAGGCTCCTGCCTCACTCCTCAAAAACGGGACAAAGGGCCAG

PHB2-DK CCTCTGGACAGGCTCCTGCCTCACTCCTCAAAAACGGGACAAAGGGCCAG

Figure 51: DNA sequencing of off-target sites in PHB1-DK and PHB2-DK cells. The top three offtarget sites for PHB1-gRNA2 (a) or PHB2-gRNA2 (b) were amplified from PHB1-DK (a) or PHB2-DK (b) genomic DNA, subcloned and sequenced using Sanger sequencing. Sequencing depth was at least $5 x$ whereas none of the sequenced amplicons contained a nucleotide different from the wildtype reference.

Taken together, both PHB1 and PHB2 were successfully targeted for endogenous tagging with the fluorescent protein Dreiklang (DK) using two different gRNAs per gene. Homologous recombination efficiencies could be increased by about 2-fold when a nuclease-resistant donor plasmids was used. Endogenous tagging of PHB1 and PHB2 resulted in 49 heterozygous PHB1-DK and 70 heterozygous PHB2-DK knock-in cells. Homozygous knockin cells were not obtained, because not every copy of the respective gene might be accessible. Total PHB1 levels in different PHB1-DK knock-in cell lines were 1.8-12.2 times higher compared to wildtype U2OS cells, while total PHB2 levels were slightly reduced (between 1 and 39\%) in various PHB2-DK clones compared to wildtype cells. The clones 
exhibiting the most native protein expression levels (1.8x PHB1 and 0.99x PHB2 compared to wildtype cells) were selected for further experiments. Endogenously tagged PHB1-DK and PHB2-DK localized to mitochondria that exhibited wildtype morphology, indicating that the previously observed aberrant mitochondrial were caused by prohibitin overexpression rather than by fluorescent protein tagging. Also, the selected knock-in cells did not contain any unwanted mutations within the surrounding sequences at the Cas9 cleavage sites or at any analyzed off-target site.

\subsubsection{Sub-mitochondrial localization of prohibitins in human cells}

Despite the insight that prohibitins are localized to the inner mitochondrial membrane (IM), little is known about their distribution within this membrane. This was probably due to a lack of specific antibodies against mammalian PHB1 or PHB2, which were not available when this study was initiated. While antibodies against protein tags could be used instead, overexpression of tagged PHB1 or PHB2 induces highly aberrant mitochondrial morphologies as shown in the previous section. Thus, the endogenously tagged PHB1-DK and PHB2-DK cell lines provided the perfect model system to study the localization of prohibitins in human mitochondria.

\subsubsection{STED microscopy reveals prohibitin clusters}

STED microscopy was used to visualize the distribution of PHB1-DK and PHB2-DK in U2OS knock-in cells. Therefore, the respective cell line was chemically fixed and decorated with a polyclonal antibody that recognizes GFP and YFP derivatives including DK. Confocal microscopy suggested that PHB1-DK and PHB2-DK are homogenously distributed over the mitochondrial network (Figure 52). However, mitochondria are small organelles that have diameters ranging from 0.2 to $1.0 \mu \mathrm{m}$ (Alberts et al., 2014). Insight about the nanoscale distribution of PHB1-DK and PHB2-DK in knock-in cells was obtained using STED superresolution microscopy. In STED images, PHB1-DK and PHB2-DK were shown to be heterogeneously distributed over mitochondria and concentrated in distinct protein clusters. While individual clusters could be detected, some regions showed denser 'hotspots' of PHB clusters. Individual PHB1-DK or PHB2-DK clusters exhibited a size of about $80 \mathrm{~nm}$. The added lengths of the used primary and secondary antibodies enlarge any detected structure by about 30-35 nm (Dyba et al., 2003; Ries et al., 2012). Thus, it can be assumed that actual diameter of the observed PHB clusters is about $45-50 \mathrm{~nm}$ and that each protein cluster contains multiple PHB1-DK or PHB2-DK copies. 
a)

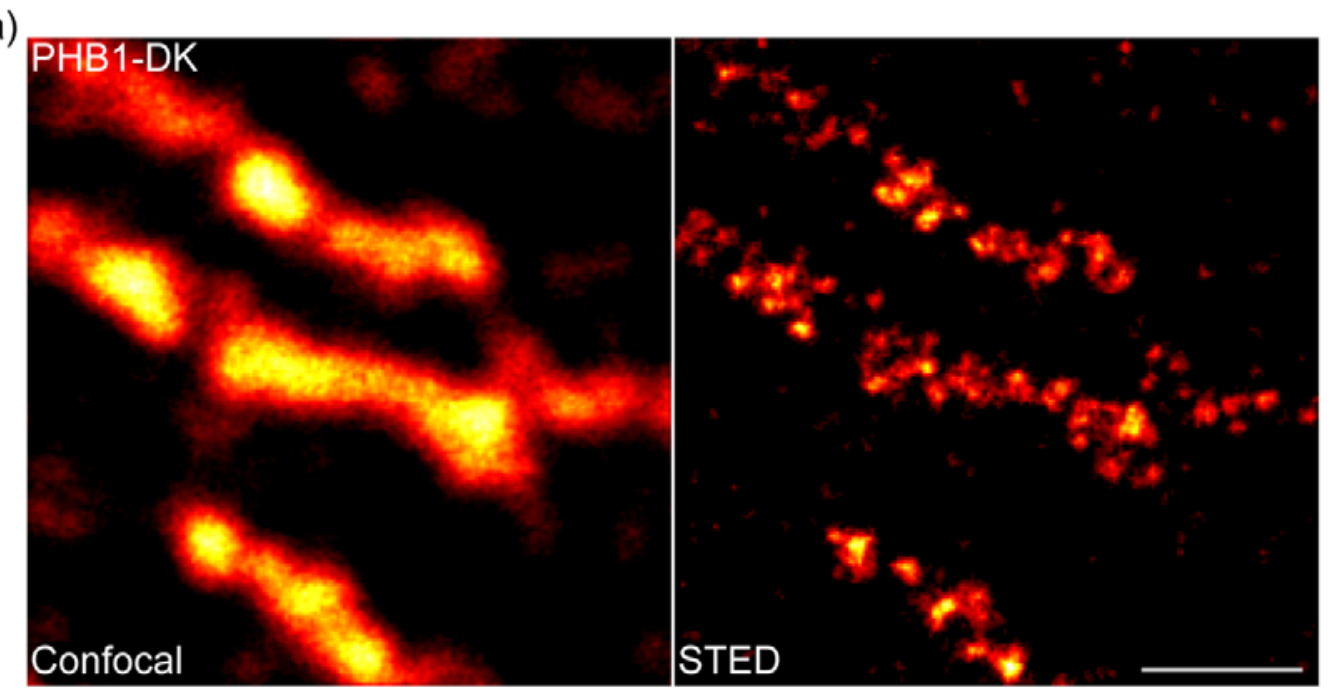

b)

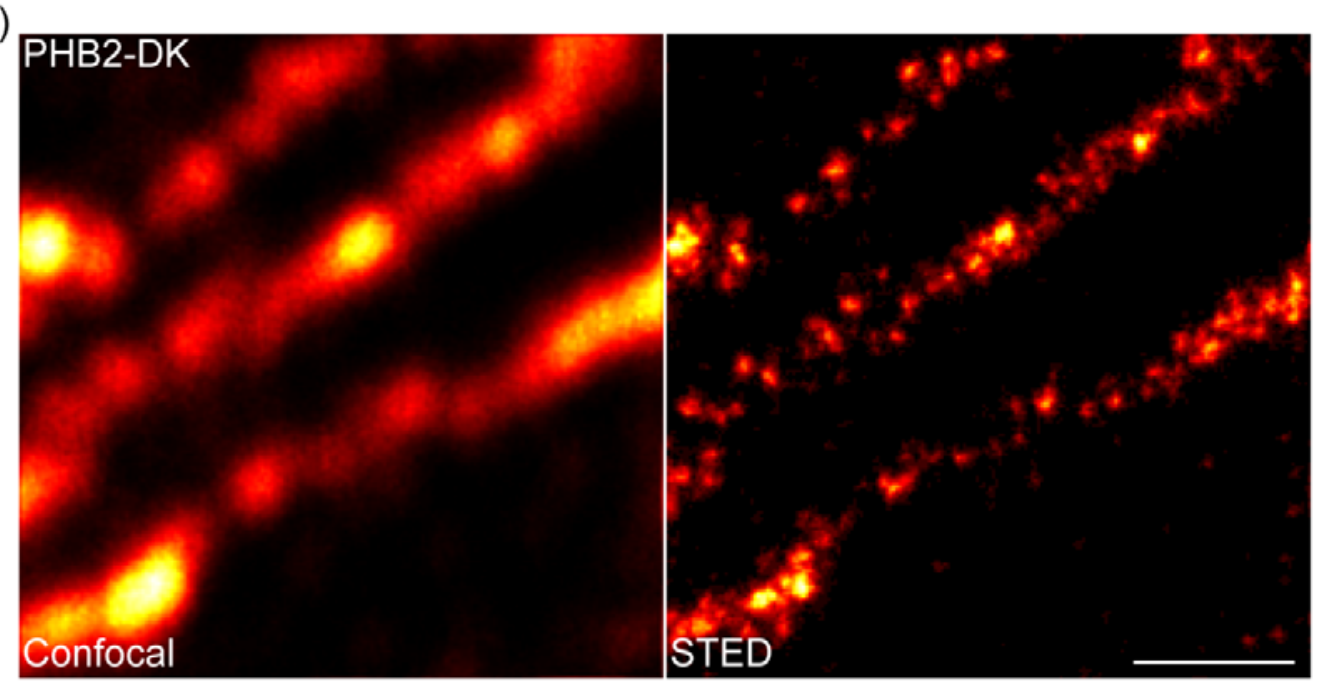

Figure 52: STED microscopy reveals PHB1-DK and PHB2-DK clusters in knock-in cells. Compared to confocal microscopy, STED microscopy enables the visualization of distinct PHB1-DK (a) and PHB2-DK (b) clusters. Endgenously tagged PHB1-DK and PHB2-DK were decorated with an antiGFP antibody to visualize the respective protein. STED images were smoothed with a low pass Gaussian filter. Scale bar: $1 \mu \mathrm{m}$.

It should be noted that fluorescent protein tagging of a protein of interest could affect the localization and functionality (Snapp, 2005). Although the fluorescent protein was placed at the C-terminal end of the respective prohibitin, which leads to successful mitochondrial import, one could argue that the observed localization in clusters was a tag-induced phenomenon. During the course of this study, two new antibodies targeting PHB1 or PHB2 became available. Therefore we used these antibodies for labeling the respective prohibitin in unmodified, wildtype human U2OS cells. STED microscopy revealed that both PHB1 and PHB2 are localized in distinct clusters that were heterogeneously distributed over 
mitochondria (Figure 53). Localization, cluster size and density of untagged and DK-tagged prohibitins were indistinguishable. Thus, it can be concluded that endogenous tagging of prohibitins does not alter their submitochondrial distribution.

a)
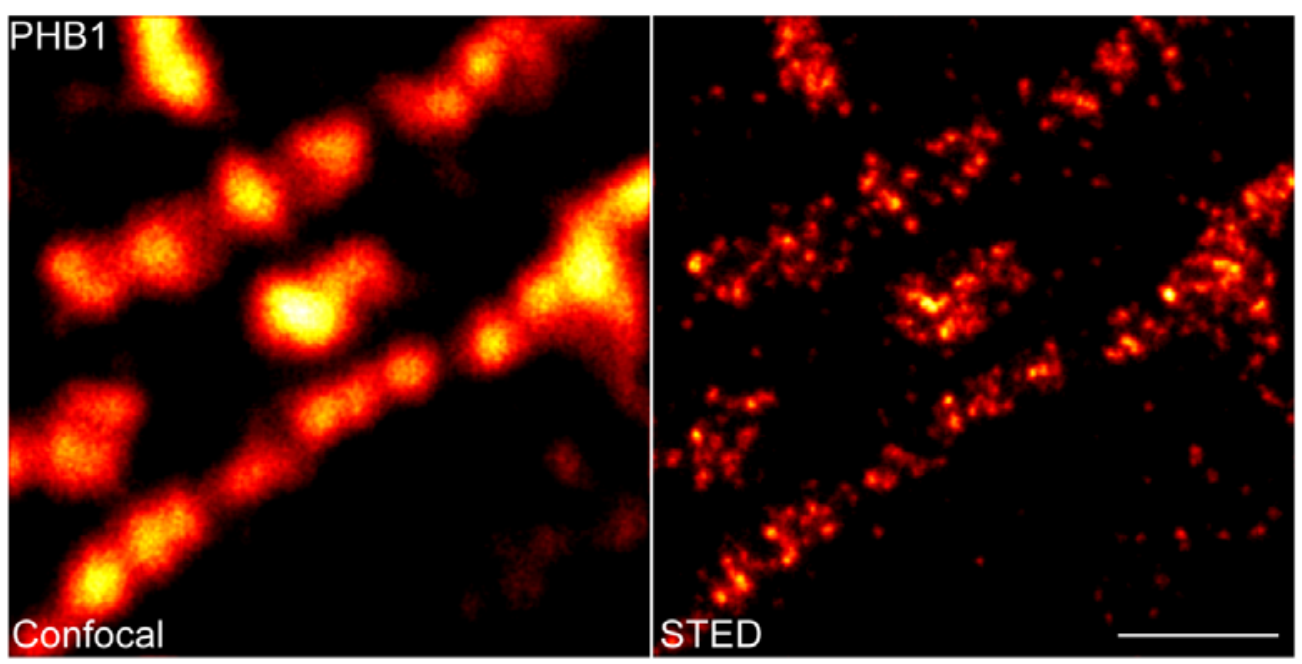

b)
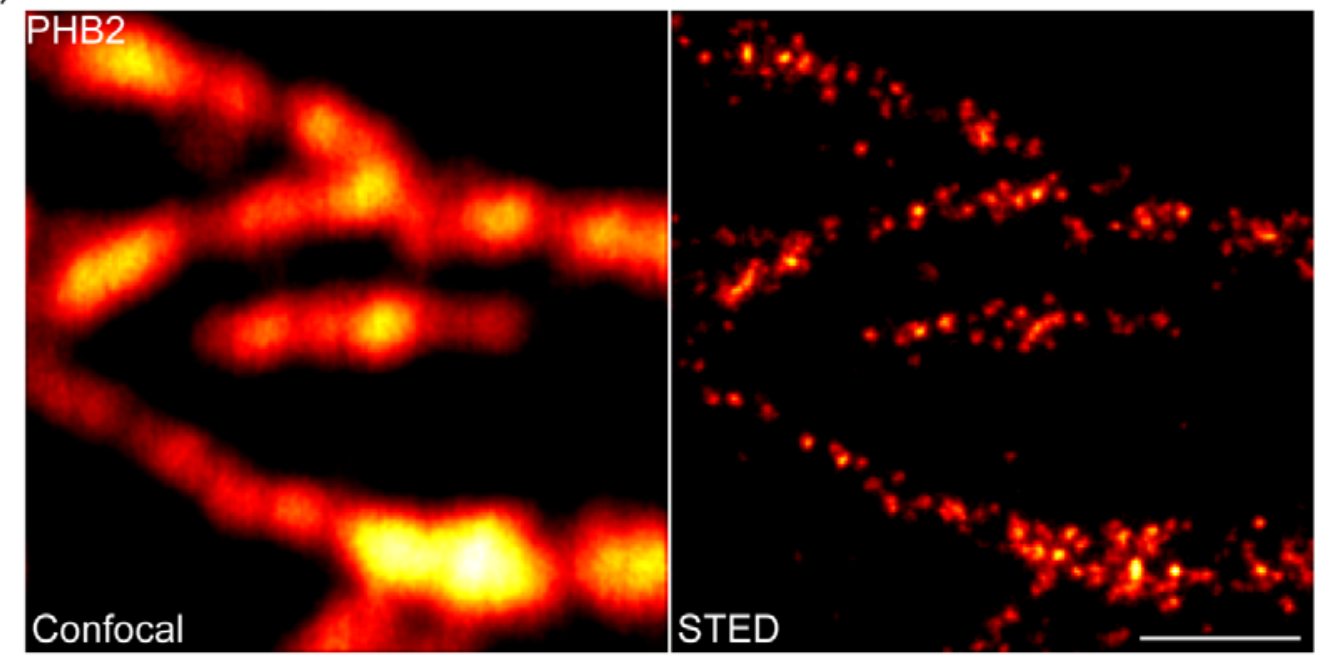

Figure 53: STED microscopy reveals PHB1 and PHB2 clusters in wildtype cells. Compared to confocal microscopy, STED microscopy enabled the visualization of distinct PHB1 (a) and PHB2 (b) protein clusters. Wildtype U2OS cells were decorated with anti-PHB1 (a) or anti-PHB2 (b) antibody, respectively. STED images were smoothed with a low pass Gaussian filter. Scale bar: $1 \mu \mathrm{m}$.

PHB1 and PHB2 are considered to form heterodimeric subunits and multiple of these subunits assemble into a high molecular weight PHB complex (Nijtmans et al., 2000, Back et al., 2002). To determine whether endogenously tagged PHB1-DK or PHB2-DK has the same distribution as untagged PHB1 or PHB2, knock-in cells were labelled with antibodies against 
GFP and the respective prohibitin (Figure 54). Intriguingly, PHB1 and PHB1-DK as well as PHB2 and PHB2-DK showed similar distribution patterns indicating a high degree of colocalization between tagged and untagged prohibitins.

a)

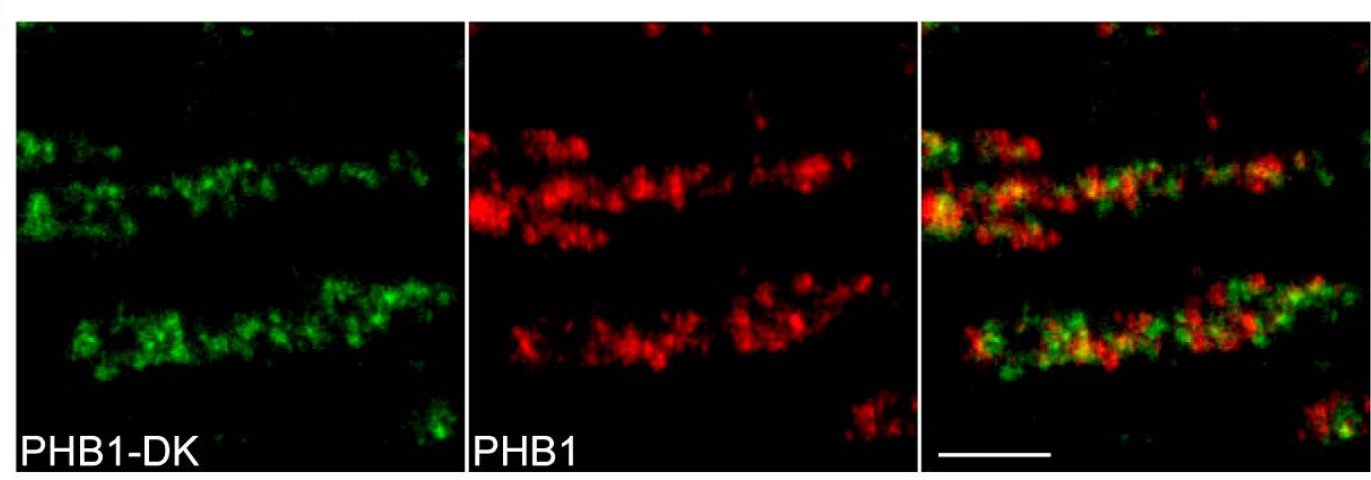

b)

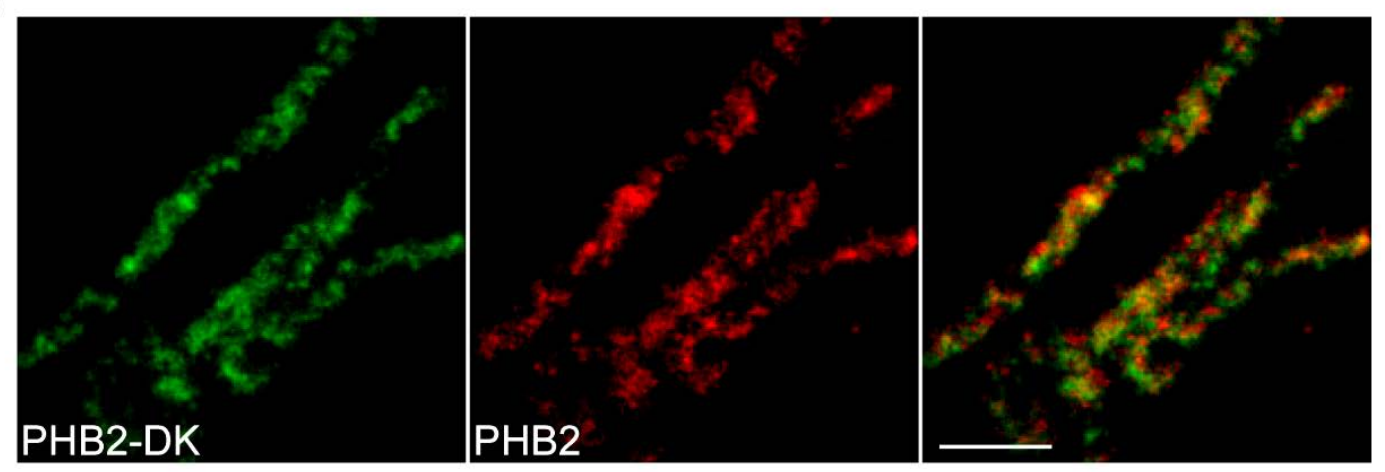

Figure 54: Co-localization of PHB1-DK with PHB1 and PHB2-DK with PHB2. STED dual-color microscopy revealed a similar distribution of tagged and untagged PHB1 (a) as well as of tagged and untagged PHB2 (b). PHB1-DK or PHB2-DK knock-in were labelled with an anti-GFP antibody and a PHB1 or PHB2 specific antibody. Images were smoothed with a low pass Gaussian filter. Scale bar: $1 \mu \mathrm{m}$.

Further, to analyze whether endogenously tagged PHB1-DK or PHB2-DK colocalizes also with untagged PHB2 or PHB1, knock-in cells were labelled with antibodies against GFP and the respective prohibitin (Figure 55). As observed in the previous experiment, a similar distribution between PHB1-DK and PHB2 or PHB2-DK and PHB1 was found.

In conclusion, both experiments showed that untagged and tagged prohibitins are largely found in the same regions of human mitochondria. This suggests that tagged prohibitins are incorporated into the PHB complex similarly to their untagged counterparts. 
a)

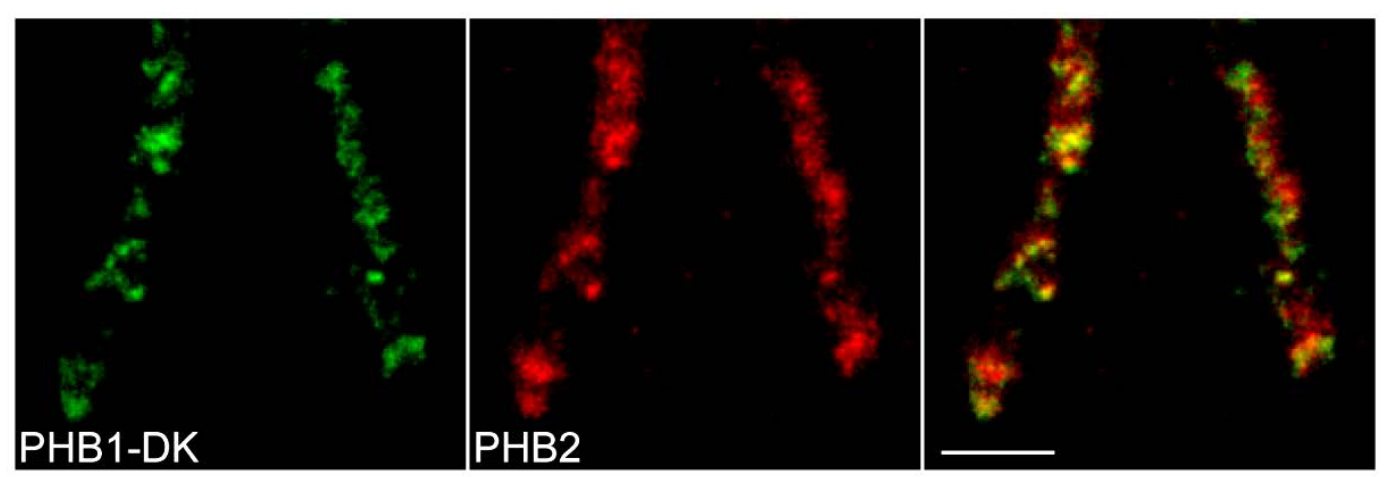

b)

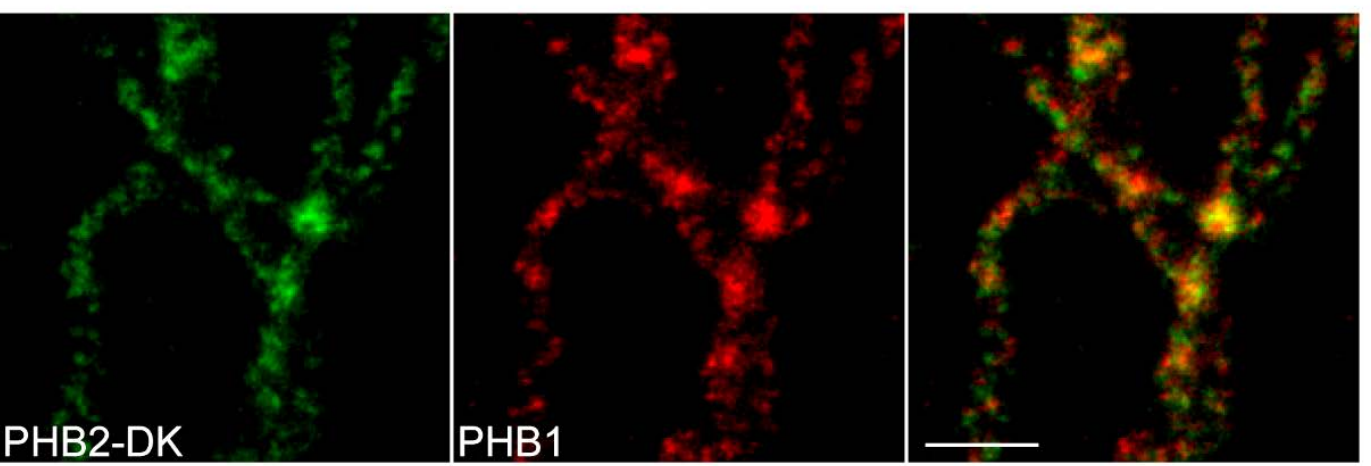

Figure 55: Co-localization of PHB1-DK with PHB2 and PHB2-DK with PHB1. STED dual-color microscopy revealed a similar distribution of PHB1-DK and PHB2 (a) as well as of PHB2-DK and PHB1 (b). PHB1-DK or PHB2-DK knock-in were labelled with an anti-GFP antibody and a PHB1 or PHB2 specific antibody. Images were smoothed with a low pass Gaussian filter. Scale bar: $1 \mu \mathrm{m}$.

\subsubsection{Immunogold EM reveals accumulation of prohibitins in cristae}

STED microscopy is a powerful tool that allowed great insights into the nanoscale organization of prohbitins in human mitochondria. However, to determine the distribution of PHB1 and PHB2 between the various subdomains of the inner membrane (IM), immunogold EM of knock-in cells was used (Figure 56). Knock-in cells were chemically fixed and cut into cryosections that were decorated with a primary anti-GFP antibody followed by protein $A$ coupled to gold particles. For quantitative analysis, the localization of each mitochondrial gold particle was measured with respect to the inner boundary membrane and the closest cristae membrane. For PHB1-DK, $85 \%$ of all gold particles $(n=106)$ were enriched at the cristae membrane (Figure 56a). Similarly, 90\% of all gold particles $(n=107)$ labeling PHB2-DK were found to be localized at the cristae membrane (Figure 56b). This is in contrary to localization of PHB1 or PHB2 in yeast cells, where both proteins seem evenly distributed within the inner membrane (Vogel et al., 2006). Therefore, the predominant cristae localization of PHB1 and PHB2 subunits might be unique to higher eukaryotes 
a)
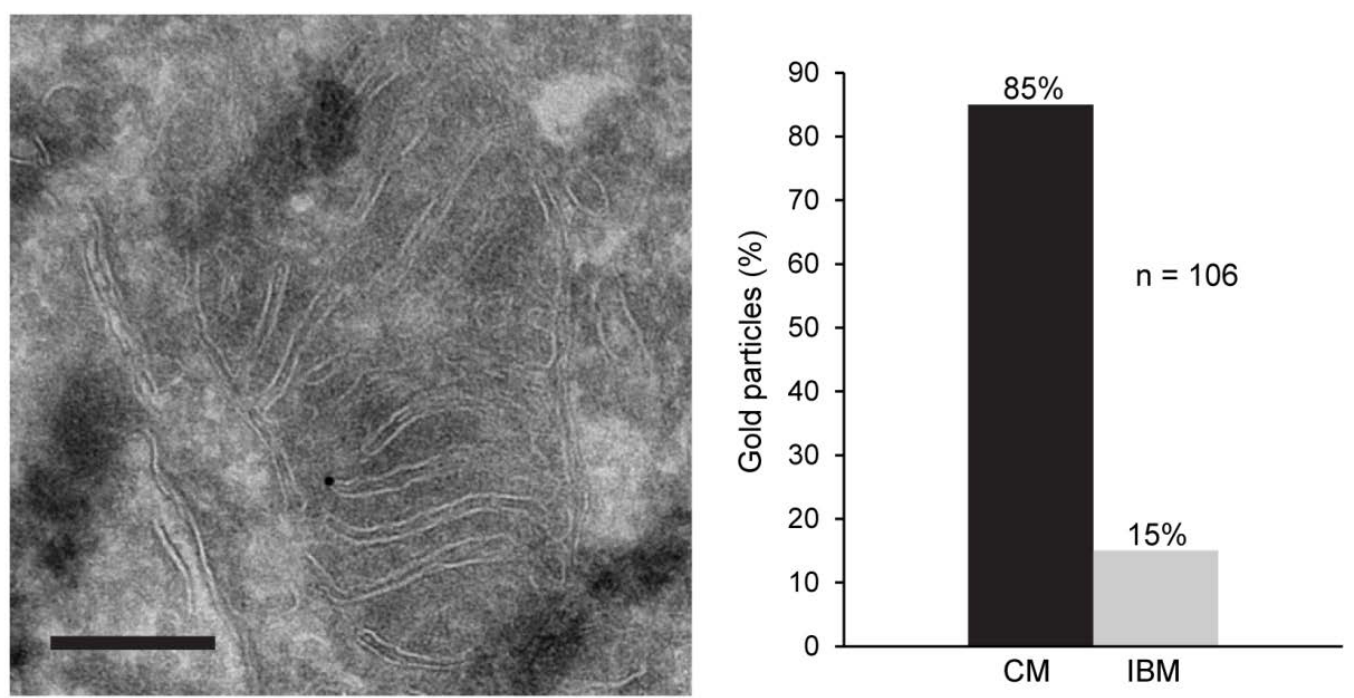

b)
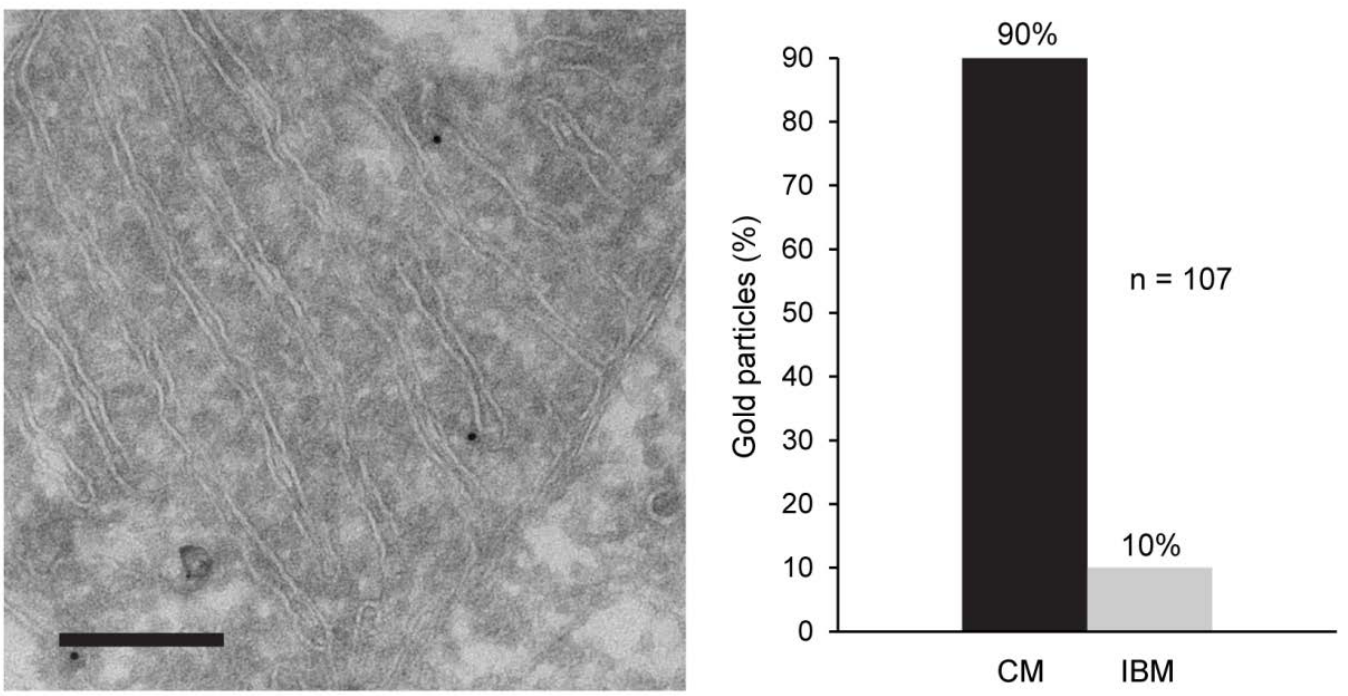

Figure 56: Submitochondrial localization of PHB1-DK and PHB2-DK using quanitative immunoelectron microscopy. Representative micrographs and their quantitative analysis are shown for immunogold labeling of PHB1-DK (a) and PHB2-DK (b) in U2OS knock-in cells. Scale bar: 200 nm.

Again, it should be noted that the submitochondrial localization of FP-tagged PHB1 or PHB2 was analyzed and that the tag itself might alter the actual localization (Snapp et al., 2005). Therefore, we used prohibitin-specific antibodies that became available recently as a control for immunogold labeling of wildtype human U2OS cells (Figure 57). 
a)
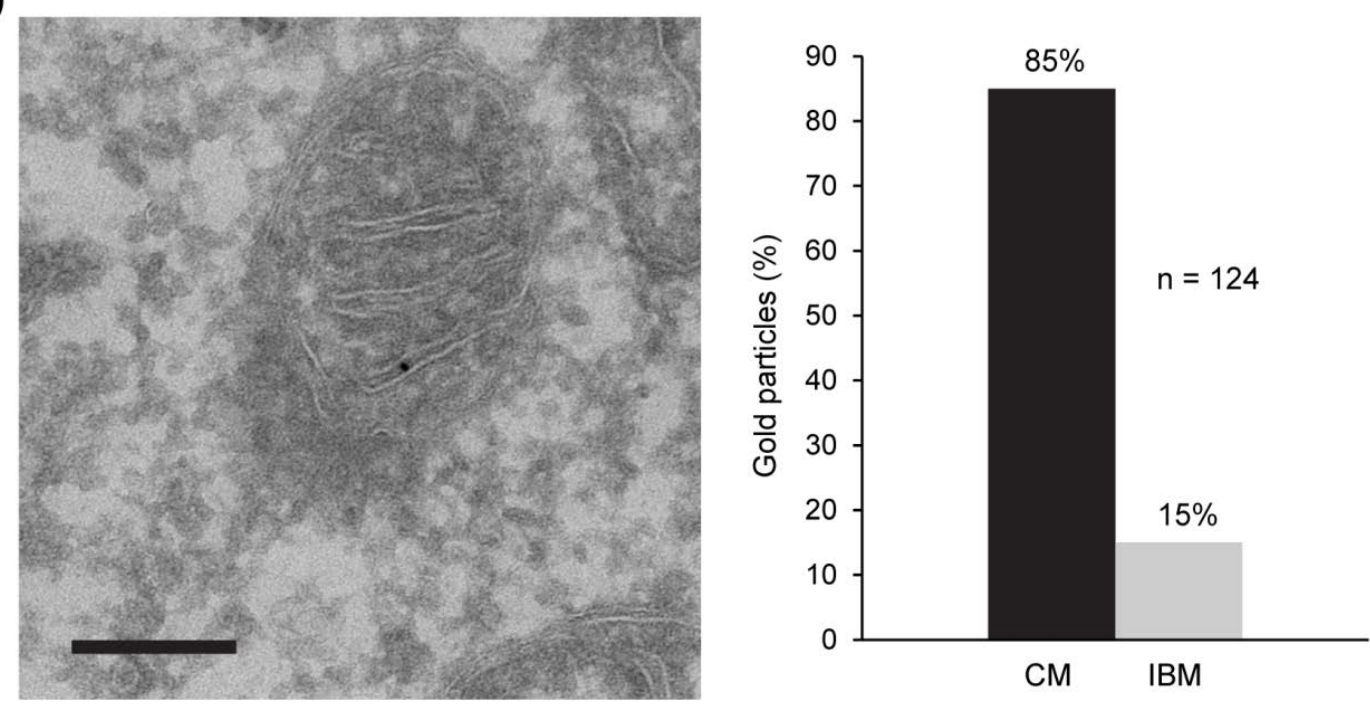

b)
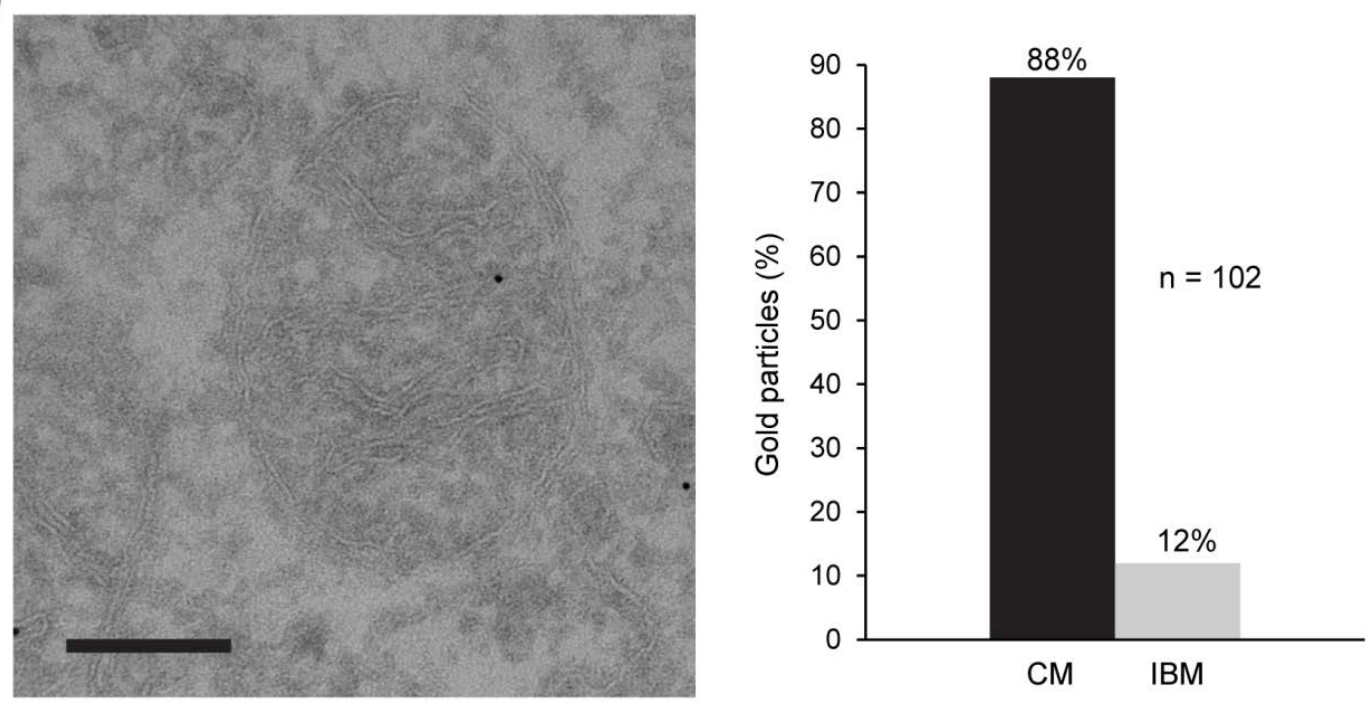

Figure 57: Submitochondrial localization of PHB1 and PHB2 in wildtype cells using quanitative Immunoelectron microscopy. Representative micrographs and their quantitative analysis are shown for immunogold labeling of PHB1 (a) and PHB2 (b) in wildtype U2OS cells. Scale bar: $200 \mathrm{~nm}$.

Similarly as observed for DK-tagged prohibitins, a mitochondrion was typically decorated with one or two gold particles. Quantification of gold particles highlighting the localization of PHB1 showed that $85 \%$ of all particles $(n=124)$ were found at the cristae membrane (Figure $57 a$ ). The fraction of cristae-localized untagged PHB1 and tagged PHB1-DK is identical indicating that PHB1 tagging does not alter its localization. Further, $88 \%$ of all gold particles $(n=102)$ decorating PHB2-labelled mitochondria were localized at the cristae membrane (Figure 57b). Thus the localization data obtained using tagged PHB2-DK could be confirmed using a PHB2 specific antibody. 
Taken together, STED microscopy revealed that PHB1-DK and PHB2-DK are localized in distinct clusters within human mitochondria. Within the inner mitochondrial membrane the vast majority of those clusters are specifically enriched to the cristae membrane as shown using immunogold electron microscopy. This localization might be a unique feature of human mitochondria as both PHB1 and PHB2 seem evenly distributed within the inner mitochondrial membrane of yeast cells (Vogel et al., 2006). Further, localization of DK-tagged PHB1 and PHB2 in distinct clusters at the cristae membrane was confirmed using prohibitin-specific antibodies.

\subsubsection{Mobility of PHB1-DK and PHB2-DK}

Endogenously tagged PHB1-DK and PHB2-DK cells provided an excellent model system to study the organization and localization of prohibitins in fixed human cells. However, information about the mobility of prohibitins within the mitochondrial inner membrane (IM) of living cells was lacking. To determine the molecular mobility of PHB1-DK or PHB2-DK, fluorescence recovery after photobleaching (FRAP) experiments were conducted (Figure 58). Living U2OS cells grown on a glass cover slip were placed in a live cell chamber for confocal microsopy analysis. Both chamber and objective were heated to $37^{\circ} \mathrm{C}$ and cells overlaid with CO2-independent L-15 medium. Mitochondrial matrix localized Dreiklang (mito-DK) was used as a reference protein. FRAP analysis of mito-DK revealed a fast recovery of fluorescence and a high mobile fraction of about 90\% (Figure 58a). This observation is in accordance with previous reports that showed a high mobility of matrix localized GFP (Partikian et al., 1998). Fluorescence recovery of PHB1-DK over a time course of one minute was slow and showed that only about $20 \%$ of PHB1-DK are mobile (Figure 58b). Similarly, the mobile fraction of PHB2-DK was found to be only 10\%. Interestingly, other FRAP studies on mitochondrial IM proteins found much higher immobile fractions of about $40 \%$ for the $\mathrm{Y}$-subunit of complex $\mathrm{V}$ and about 50\% for subunit Cox8a of complex IV (Sukhorukov et al., 2010). Those proteins appear to be almost homogenously distributed between inner boundary (IBM) and cristae membrane (CM) (Vogel et al., 2006) and it was suggested that the immobile fraction of $\mathrm{Y}^{-}$ subunit and Cox8a reflects the cristae-localized fraction of these molecules (Sukhorukov et al., 2010). This is in agreement with the FRAP data acquired for PHB1-DK and PHB2-DK. As about $90 \%$ of both proteins are enriched in the CM, a similarly high fraction of both PHB1-DK and PHB2-DK is essentially immobile in the cristae membrane. Interestingly, it was proposed that prohibitins form microdomains within the mitochondrial IM (Osman et al., 2009a). While such domains might exhibit a low molecular mobility, FRAP is not well suited for studying 
microdomains, because they are usually smaller than the resolution of the used confocal microscope (Pike, 2009). Instead, more advanced optical techniques based on singlemolecule tracking or super-resolution microscopy would be required (Douglass and Vale, 2005; Eggeling, 2015).

Taken together, the low mobility of PHB1-DK and PHB2-DK is in agreement with their role as subunits of a high molecular weight complex that is mainly found within the CM of human mitochondria.

a)
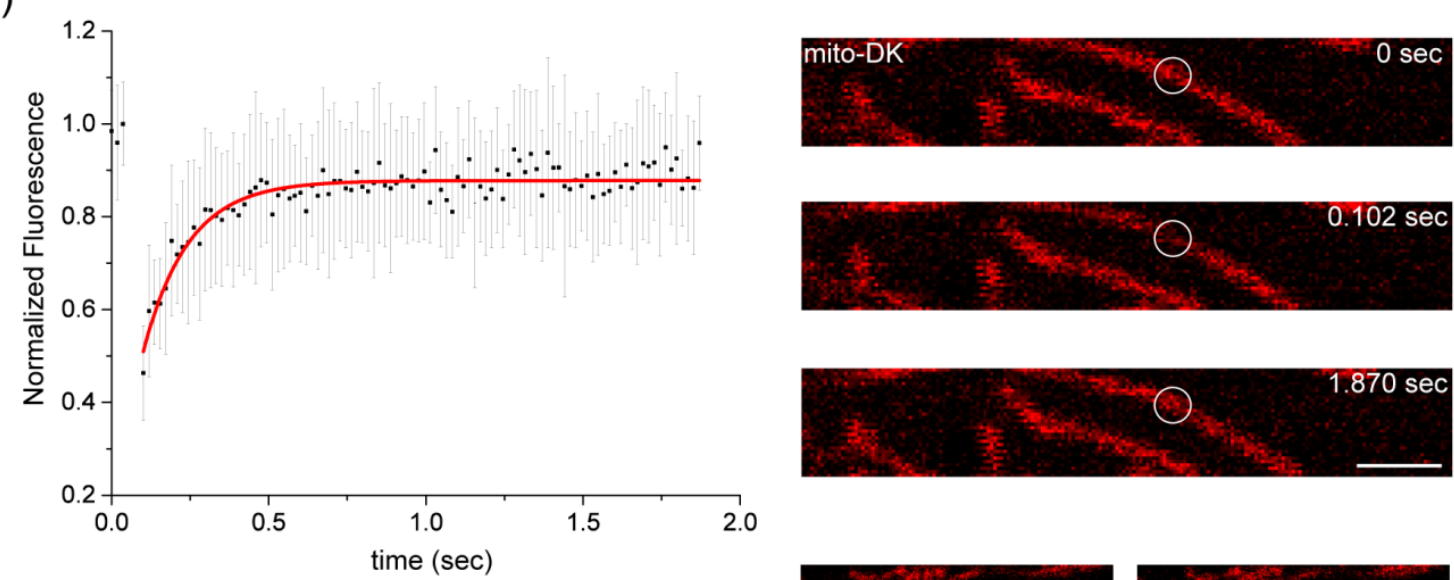

b)
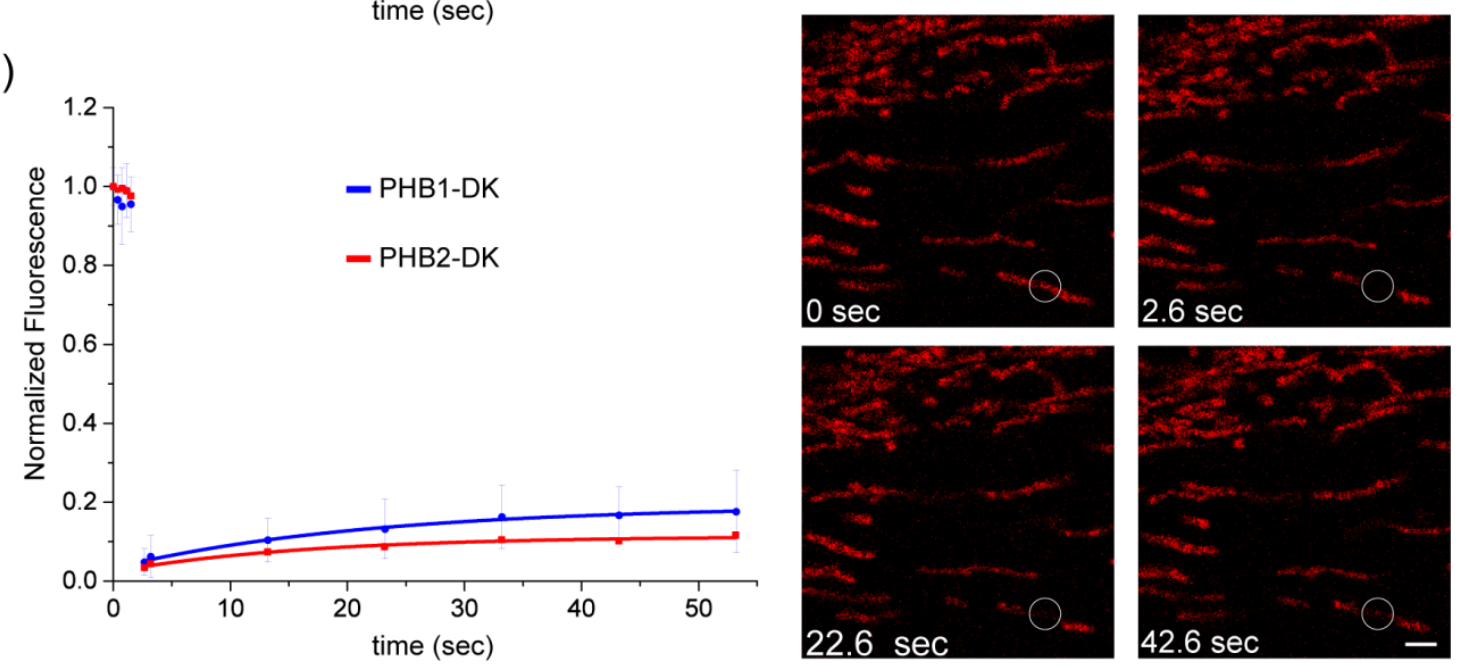

Figure 58: PHB1-DK and PHB2-DK are localized in immobile fractions. a) Matrix-localized Dreiklang (mito-DK) is highly dynamic with a mobile fraction of about $90 \%$. b) The mobile fraction of PHB1-DK (blue graph) is about 20\% whereas the mobile fraction of PHB2-DK (red graph) is about $10 \%$. Each data point is the average of multiple experiments (mito-DK, $\mathrm{n}=17$; PHB1-DK, $\mathrm{n}=18$; PHB2-DK, $n=20$ ). The data were fitted with an exponential function and error bars denote SD. White circles indicate regions of interest $(\mathrm{ROI})$ that were selectively bleached. Scale bar: $2 \mu \mathrm{m}$. 


\subsubsection{Nuclear localization of PHB2 in HeLa cells is an overexpression artefact}

Previous studies in HeLa cells showed an estradiol (E2) and estrogen receptor (ESR1) dependent nuclear localization of PHB2 (Kasashima et al., 2006). In presence of both ESR1 and E2, overexpressed PHB2-GFP was found to accumulate in the nucleus and an export from mitochondria was suggested, because cytosolic translation was blocked. As mislocalization upon overexpression is a common artefact, we set out to reproduce the mentioned experiments in endogenously tagged cells. First, HeLa cells expressing a genomically tagged version of PHB2 had to be generated. Therefore, PHB2-gRNA2 and the nuclease-resistant donor plasmid PHB2-DK were used as shown for U2OS cells previously (section 1.4.2). Seven days after plasmid co-transfection, the cells were analyzed and individually sorted into two 96-well plates using FACS (Figure 59). The fraction of fluorescently tagged HeLa cells was $11.4 \%$, which is similar to the results obtained for PHB2tagging in U2OS cells (10\%). This was expected, because both cell lines are of human origin and therefore a similar genomic sequence.
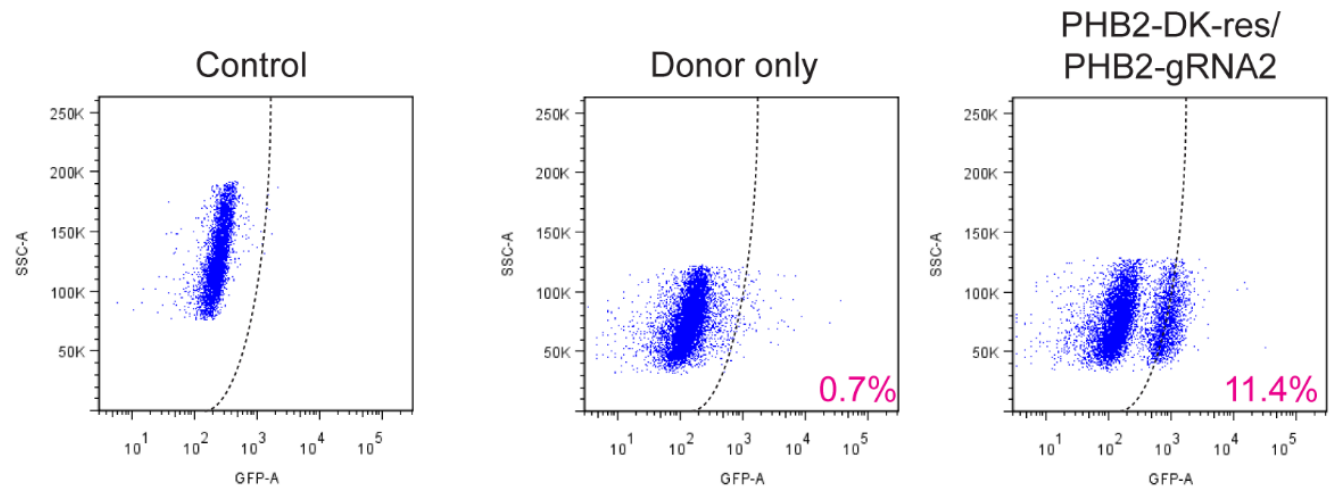

Figure 59: CRISPR-mediated endogenous PHB2-tagging in HeLa cells. Co-transfection of HeLa cells was done with PHB2-gRNA2 and the Cas9-resistant PHB2-DK donor plasmid yielding 11.4\% DK+ cells. Wildtype HeLa cells and donor plasmid only transfected cells were used as a control.

Two 96-well plates were sorted initially and a total amount of 26 HeLa cell lines was obtained after about two weeks. Analysis of those cells was done using out-out PCR and showed that 25 out of 26 clones were heterozygous (Figure 60). This is in accordance with the result obtained for U2OS cells, where all knock-in cells were heterozygous for transgene integration at the PHB2 locus. 
PHB2-DK clone \#

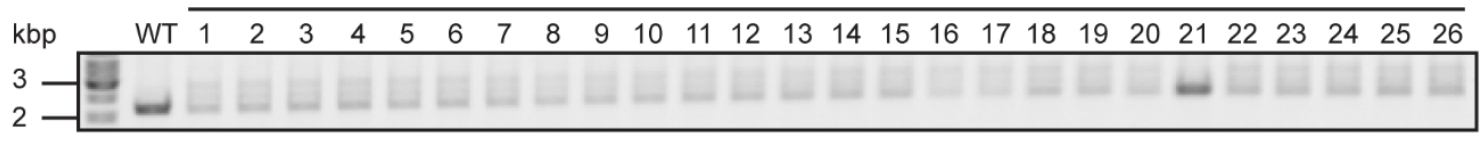

Figure 60: Genotyping of HeLa-PHB2-Dreiklang clones using PHB2 locus-specific out-out PCR. Genomic DNA was isolated and used as a template for PCR with primers that anneal outside of the homology regions of the donor plasmid.

To determine whether nuclear translocation of PHB2 also occurs in endogenously tagged HeLa cells, one clone was picked randomly for further experiments. Previously, PHB2 localization within the nucleus was observed upon co-expression of both PHB2-GFP and ESR1 in wildtype HeLa cells (Kasashima et al., 2006). Therefore, wildtype cells cotransfected with tagged PHB2-DK and untagged ESR1 served as a control. Further, endogenously tagged PHB2-DK cells were transfected with the same amount of ESR1 plasmid. Importantly, the PHB2 plasmid was replaced with the same amount of plasmid backbone DNA to match total DNA levels in the transfection mixture and to obtain similar transfection efficiencies.

One day after co-transfection of wildtype HeLa cells with PHB2-DK/ESR1, the cells were treated with E2 for $2 \mathrm{~h}$, fixed and incubated with an antibody detecting ESR1 as described (Kasashima et al., 2006). A secondary antibody conjugated to the red fluorophore KK114 (Kolmakov et al., 2010) was used for ESR1 visualization. Intrinsic DK fluorescence was detected for analysis of PHB2-DK distribution. Indeed, some of the co-transfected HeLa cells showed a nuclear localization of PHB2-DK (Figure 61a, I). However, these cells also displayed fragmented mitochondria or cytoplasmic PHB2-DK aggregates, a phenotype previously observed in U2OS cells, which overexpress PHB2-DK (section 1.4.1). Moreover, the majority of HeLa cells expressing PHB2-DK and ESR1 displayed a mitochondrial localization of PHB2-DK (Figure 61a, II). The same experiment was done using endogenously tagged PHB2-DK cells transfected with an ESR1-encoding plasmid. Interestingly, while overexpressed ESR1 was found in the nucleus, endogenously tagged PHB2-DK was exclusively localized to mitochondria (Figure 61b). 
a)
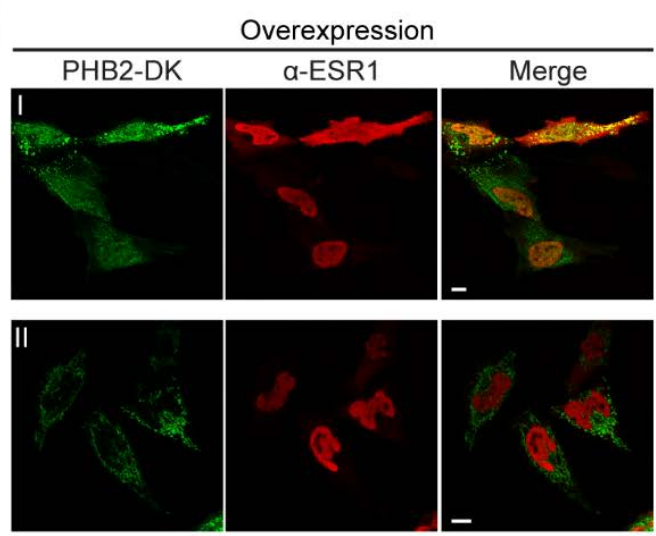

b)

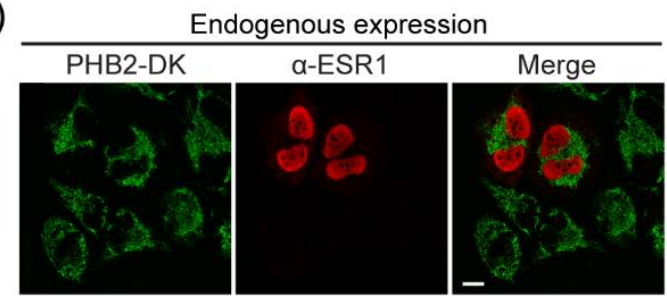

Figure 61: PHB2 overexpression induces nuclear mislocalization in HeLa cells. a) Overexpression of ESR1 and PHB2-DK induces a heterogenous localization of PHB2 in both nucleus (I) and mitochondria (II). b) Endogenously expressed PHB2-DK is solely localized in mitochondria upon overexpression of ESR1.

Taken together, nuclear localization of PHB2 is not a general phenomenon, because it only occurs in a fraction of co-transfected PHB2-DK/ESR1 cells. Further, co-localization of ESR1 and PHB2-DK in the nucleus was only observed in those cells with highly aberrant mitochondrial morphology and cytoplasmic PHB2-DK aggregates. Because endogenously tagged PHB2-DK was exclusively found in mitochondria in presence of ESR1, it can be concluded that nuclear localization of PHB2-DK is induced by artificially high protein levels that result from plasmid-based overexpression. It should be noted that this observation is specific for HeLa cells. While the accumulation of PHB2 in the nucleus of HeLa cells seems to be an overexpression artefact, it might well be the case that PHB2 and/or PHB1 are localized to the nucleus or the plasma membrane in other cell types or tissues (Mishra et al., 2005; Mishra et al., 2006).

\subsubsection{Prohibitin quantification in single cells and cristae membranes}

Based on in vitro chemical crosslinking and mass spectrometry of purified prohbitins from yeast, it was suggested that a PHB1-PHB2 heterodimer forms the structural building block of the PHB complex (Back et al., 2002). Prohibitins were also proposed to form ring-like structures with an outer diameter of 20-25 nm using single particle electron microscopy of purified yeast PHB complexes (Tatsuta et al., 2005). Interestingly, without actually determining the copy number, these studies concluded that the 1-1.4 MDa large PHB complex should contain 12-16 (Back et al., 2002) or 16-20 (Tatsuta et al., 2005) copies of each PHB1 and PHB2 subunit. However, data regarding the structural organization of the 
human PHB complex and its stoichiometric organization are completely lacking. Therefore, analysis of PHB1 and PHB2 copy numbers in genome-edited and in wildtype human cells was done based on quantitative western blotting.

\subsubsection{Quantitative western blotting using recombinant Dreiklang as standard}

Ideally, quantification of absolute protein numbers per cell, organelle or protein complex is done using fluorescence microscopy, because of its high sensitivity and the obtained spatial information (Coffman and $\mathrm{Wu}, 2014$; Ulbrich, 2015). However, this approach relies on endogenously tagged yeast or human cells where every protein of interest is expressed as an FP fusion. As the generated PHB1-DK and PHB2-DK knock-in cell lines are heterozygous for the tagged allele, determining the number of fluorescent PHB1-DK or PHB2-DK proteins per cell would lead to an underestimation of the true protein amount. Therefore we set out to determine the average number of each prohibitin molecule per cell via quantitative western blot analysis. Importantly, this approach does not rely on the availability of purified proteins of interest commonly used for a calibration curve. It simply requires the availability of purified fluorescent protein used for endogenous tagging as well as an antibody directed against the protein of interest.

For quantification of the total amount of PHB1 and PHB2 per cell, the heterozygous PHB1-DK and PHB2-DK cell lines were used. Recombinant DK was expressed and purified from E. coli cells to high purity using affinity and size exclusion chromatography. Together with PHB1-DK or PHB2-DK cell lysates corresponding to a known number of cells, various DK amounts were separated on a SDS gel and transferred to a membrane for western blot analysis (Figure 62). First, visualization of protein bands that correspond to purified DK as well as PHB1-DK or PHB2-DK fusion protein was achieved by membrane decoration with a polyclonal anti-GFP antibody. A calibration curve was obtained by plotting the background-subtracted integrated intensities measured for each DK band. The amount of tagged PHB1-DK or PHB2-DK was determined by comparing the background-subtracted intensities of the respective bands to the DK standard curve. Subsequently, the membrane was stripped and incubated with the antibody specifically recognizing PHB1 or PHB2, respectively. This step visualized both the tagged and untagged version of each prohibitin. Importantly, the amount of tagged PHB1-DK or PHB2-DK is known from the previous step and this information is now used to determine the amount of untagged prohibitin. First, the ratio between the known amount of PHB1-DK or PHB2-DK and the corresponding band intensity after incubation with the anti-PHB1 or antiPHB2 antibody is calculated. By multiplying this ratio with the band intensity of the respective 
untagged prohibitin, the amount of untagged prohibitin is easily determined. Calculation of tagged or untagged prohibitin per cell was done by taking their specific molecular weights and the number of cells used as input into account.

a)

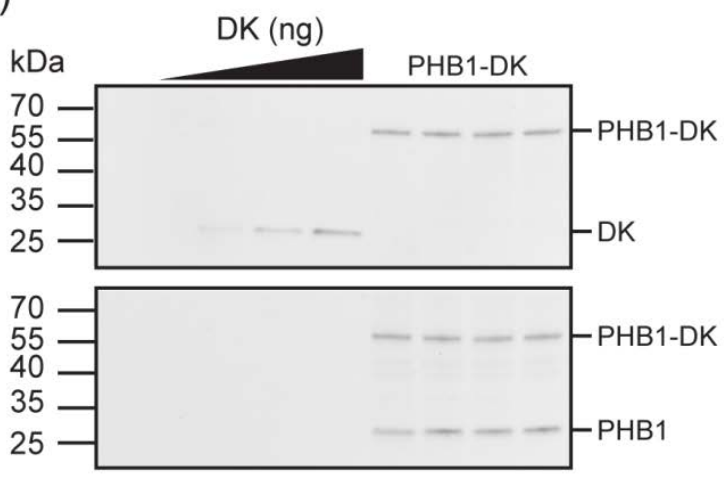

b)

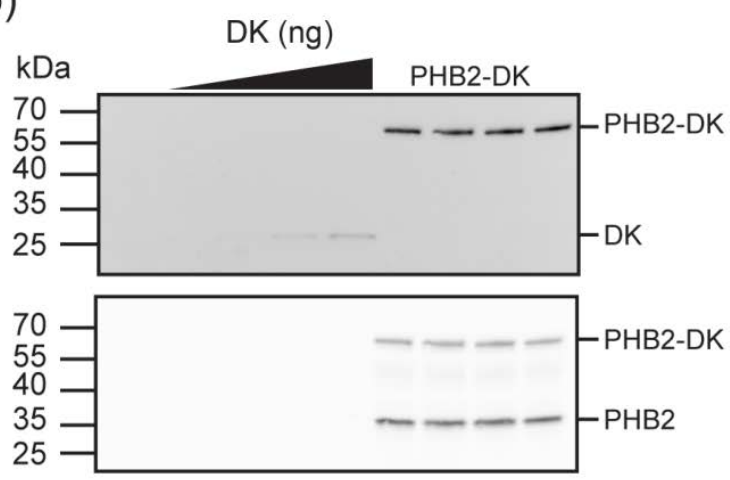

c)

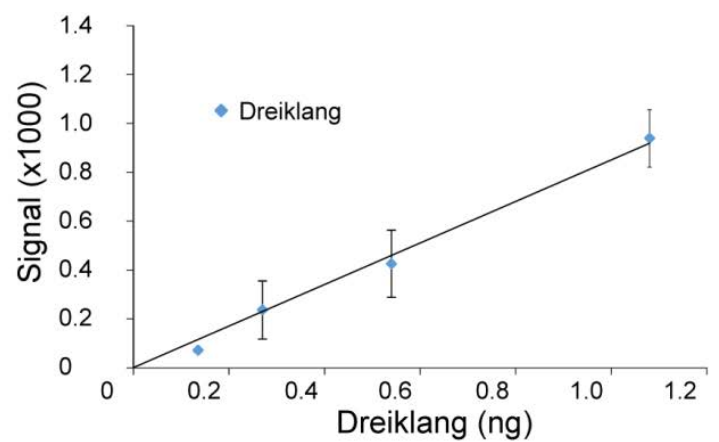

Figure 62: Estimation of PHB1 and PHB2 amounts per cell using recombinant DK. a) A Dreiklang (DK) calibration curve was generated using various amounts of recombinant DK. The membrane was probed against both DK followed by PHB1. The estimated amount of total PHB1 molecules per cell was $2.22 \pm 0.26 \times 10^{6}$. b) A DK calibration curve was generated using various amounts of recombinant DK. The membrane was probed against both DK followed by PHB2. The estimated amount of total PHB2 molecules per cell was $1.07 \pm 0.09 \times 10^{7}$. c) DK calibration curve obtained from band intensity values from a) and $b$ ).

This analysis revealed that each U2OS cell contains about $7.10 \pm 0.25 \times 10^{5}$ PHB1-DK and $1.51 \pm 0.24 \times 10^{6} \mathrm{PHB} 1$ molecules. Thus, the total of PHB1 containing molecules per cell was $2.22 \pm 0.26 \times 10^{6}$. Further, it was found that each cell contains about $2.09 \pm 0.08 \times 10^{6} \mathrm{PHB} 2-$ DK molecules and $8.59 \pm 0.83 \times 10^{6}$ PHB2 molecules. In total, this sums up to $1.07 \pm 0.09 \times$ $10^{7}$ PHB2 containing molecules per genome-edited U2OS cell.

In conclusion, the number of PHB2 molecules is about 5 times higher than the number of PHB1 molecules. This was surprising, because both prohibitins were reported to assemble stoichiometrically into a PHB complex that contains equal amounts of each PHB1 and PHB2 
subunit (Back et al., 2002; Tatsuta et al., 2005). However, the here presented numbers were determined in genomically tagged cells and fluorescent protein tagging or undetected mutations in gene regulatory regions due to gRNA treatment might induce changes in protein expression levels. Therefore, a control quantification experiment was repeated for wildtype U2OS cells. Because those cells do not express endogenously DK-tagged prohibitins, recombinant prohibitins instead of recombinant DK were used as a standard.

\subsubsection{Quantitative western blotting using recombinant prohibitins as standard}

Analysis of prohibitin levels in wildtype U2OS cells was done using recombinant N-terminally 6xHis-tagged PHB1 or PHB2 purified from E. coli (Abcam, Cambridge, UK) as a standard. Together with wildtype cell extracts, different amounts of recombinant PHB1 or PHB2 were separated on a SDS gel and transferred onto a nitrocellulose membrane. Detection of recombinant and endogenous PHB1 was done using an anti-PHB1 antibody (Figure 63a). Estimation of unknown PHB1 amounts in cell extracts was easily done by comparing signal intensities of the detected PHB1 band to the generated calibration curve. This resulted in an average amount of $8.21 \pm 0.38 \times 10^{6} \mathrm{PHB} 1$ molecules per cell. Detection of PHB2 in recombinant protein preparations and cell extracts was done using an anti-PHB2 antibody (Figure 63b). The estimated amount of PHB2 molecules per cell was $3.57 \pm 0.20 \times 10^{7}$. In conclusion, the number of PHB2 molecules was about 3-5 times higher than the number of PHB1 molecules per single U2OS cell.

a)

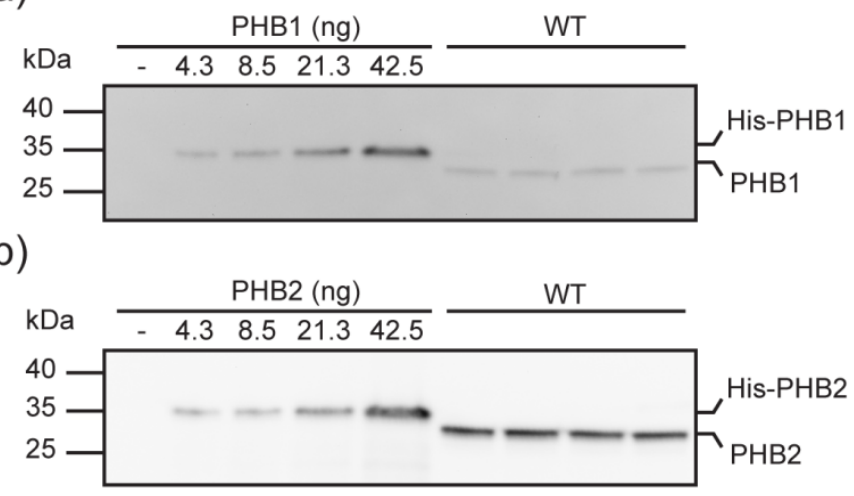

c)

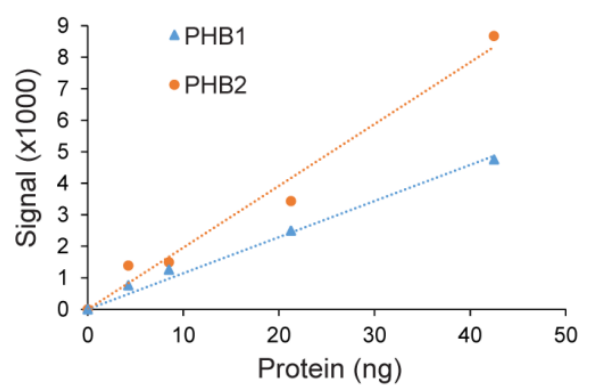

Figure 63: Estimation of PHB1 and PHB2 amounts per cell using recombinant PHB1 or PHB2 for calibration. Increasing amounts of 6xHis-PHB1 (a) or 6xHis-PHB2 (b) were loaded together with wildtype U2OS cell extracts for antibody-based detection of PHB1 (a) or PHB2 (b). The amount of PHB1 was $8.21 \pm 0.38 \times 10^{6}$ molecules per cell. The estimated PHB2 amount was $3.57 \pm 0.20 \times 10^{7}$ molecules per cell. Note that recombinant PHB1 and PHB2 run at a higher MW than endogenous ones due to the His-tag and linker sequence. c) 6xHis-PHB1 and 6xHis-PHB2 calibration curves showing a linear relationship between protein amount and signal intensity. 
Quantitative western blotting using recombinant DK for calibration revealed that the total amount of PHB1 molecules per genome-edited U2OS cell was $2.22 \pm 0.26 \times 10^{6}$. Using recombinant 6xHis-PHB1 for calibration, an average amount of $8.21 \pm 0.38 \times 10^{6} \mathrm{PHB} 1$ molecules per wildtype U2OS cell was estimated. The total amount of PHB2 was found to be about $1.07 \pm 0.09 \times 10^{7}$ molecules per genome-edited cell when recombinant DK was used for calibration. This number increased to $3.57 \pm 0.20 \times 10^{7}$ PHB2 molecules per wildtype cell when recombinant 6xHis-PHB2 was used for calibration. From a technical point of view these data suggest that both quantification methods are valid as they yield similar results. Interestingly, a 3.6-fold increase in molecule numbers was found for both PHB1 and PHB2 samples when the calibration curve was done using the corresponding recombinant protein. The concentration of commercially obtained recombinant PHB1 or PHB2 was confirmed using the bicinchoninic acid (BCA) assay (Smith et al., 1985). However, partial degradation of the respective recombinant prohibitin might have occurred during handling, which leads to overestimation of the actual protein amount on the gel.

In conclusion, both quantification methods showed that the number of PHB2 molecules exceeds that of PHB1 molecules by a factor of 4-5. This was unexpected, because both prohibitins were reported to assemble stoichiometrically into a PHB complex that consists of 12-16 (Back et al., 2002) or 16-20 (Tatsuta et al., 2005) copies of each PHB1 and PHB2 subunit. However, those numbers are based on in vitro structural analysis of purified yeast prohibitins (Back et al., 2002; Tatsuta et al., 2005) and comparable data on the mammalian $\mathrm{PHB}$ complex are lacking. Therefore, it is conceivable that the in vivo organization of the PHB complex in human cells differs.

\subsubsection{Mapping of prohibitins to individual cristae}

Independent of their exact molecular stoichiometry, it can be assumed that the amount of PHB2 is 4-5 times higher than that of PHB1 in human U2OS cells. Using electron microscopy analysis, it was shown that $85-90 \%$ of both PHB1 and PHB2 are localized to the cristae membrane (Figure 56 and 57). It would be highly interesting to use this information to estimate the average amount of prohibitin molecules per cristae in individual mitochondria of human U2OS cells. However, determining the exact number of mitochondria in a human cell is difficult as these organelles constantly undergo fusion and fission (Westermann, 2010; Youle and van der Bliek, 2012). Therefore, a combination of light and electron microscopy 
was used to first estimate the total length of the mitochondrial network per cell followed by EM to determine the amount of cristae membranes per length unit.

The mitochondrial network in human U2OS cells was stained using Mitotracker ${ }_{\circledast}$ Deep Red FM. Instead of trying to analyze the amount of distinct mitochondrial particles, the average length of the entire mitochondrial network per cell was estimated (Figure 64). Therefore, single cells exhibiting mitochondrial tubules that could be distinguished from neighboring tubules were chosen. After thresholding and image binarization, an image skeletonization algorithm for length measurements was applied (Niemisto et al., 2005). Skeletonization resulted in a morphological skeleton representation of the mitochondrial network that preserves the extent and connectivity of the original image. The resulting skeleton was used for total length measurements of mitochondrial tubules. This analysis revealed that a single human U2OS cell possesses a total mitochondrial network length of $937 \pm 284 \mu \mathrm{m}(\mathrm{SD}, \mathrm{n}=$ 81 cells). The size variability is in agreement with previous studies showing that morphological heterogeneity is a general feature of mitochondria (Collins et al., 2002; Kuznetsov et al., 2004).
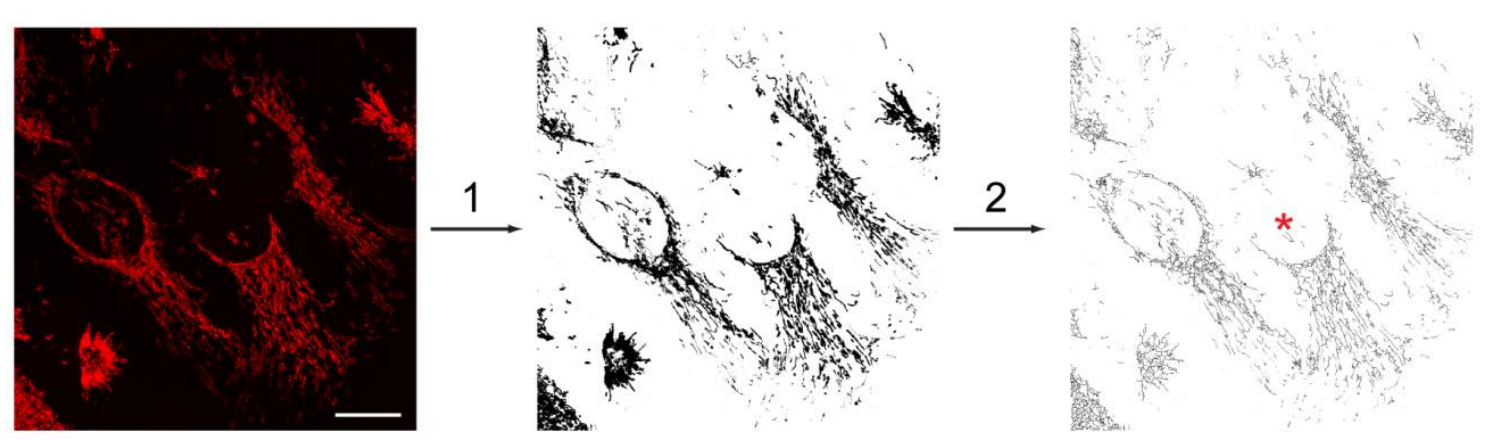

|3 $1090 \mu \mathrm{m}$
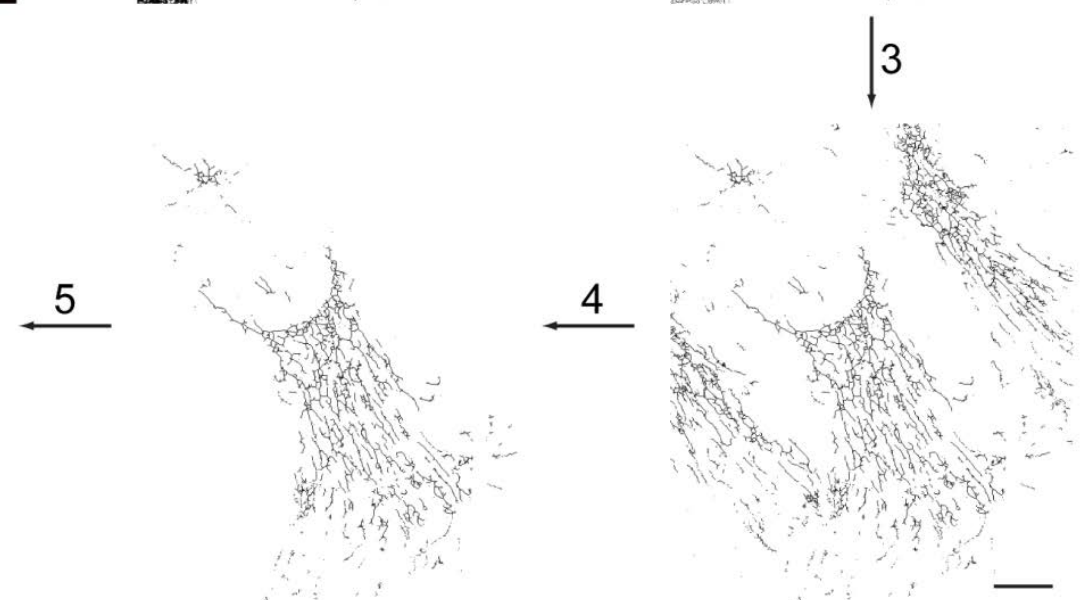

Figure 64: Determination of mitochondrial network length. A representative fluorescence image shows mitochondria in U2OS cells visualized using Mitotracker Deep Red FM. Analysis of total 
mitochondria network length in single cells was done as follows: image binarization (1), image skeletonization (2), cell selection (3, red asterisk), clearing of area outside cell of interest (4) and skeleton length measurement (5). The final mitochondrial network length for the selected cell was 1090 $\mu \mathrm{m}$. Skeleton length measurement was done using the algorithm from Niemistö et al. (2005). Scale bar: $20 \mu \mathrm{m}$.

Electron microscopy of human cells was used to estimate the number of cristae per mitochondrion. Therefore human U2OS cells were grown to sub-confluency on Aclar cover slips and gently fixed on the growth surface. After additional fixation, contrasting and dehydration steps, the cells were embedded in Epon (Agar 100). Embedded cells were sectioned by cutting parallel to the growth surface. This procedure ensured that mitochondrial tubules are sectioned along their longitudinal axis thus allowing the visualization of distinct cristae membranes. A representative micrograph obtained using the approach is depicted in Figure 65a. Individual mitochondria with clearly visible cristae membranes were chosen for analysis. Here the distance between two neighboring cristae was measured at different positions (Figure 65b). After analysis of a number of mitochondria the average inter-cristae distance was calculated with $80 \pm 19 \mathrm{~nm}$ (SD, $\mathrm{n}=721$ distances).

a)

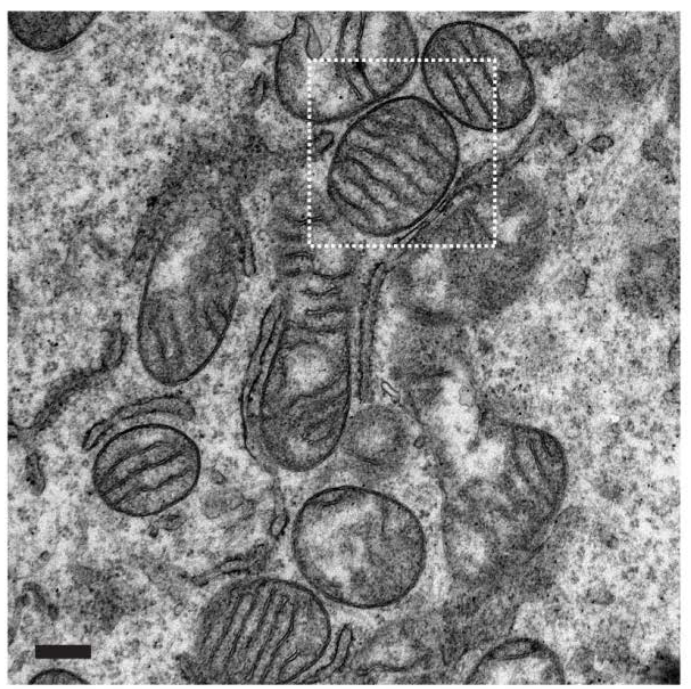

b)

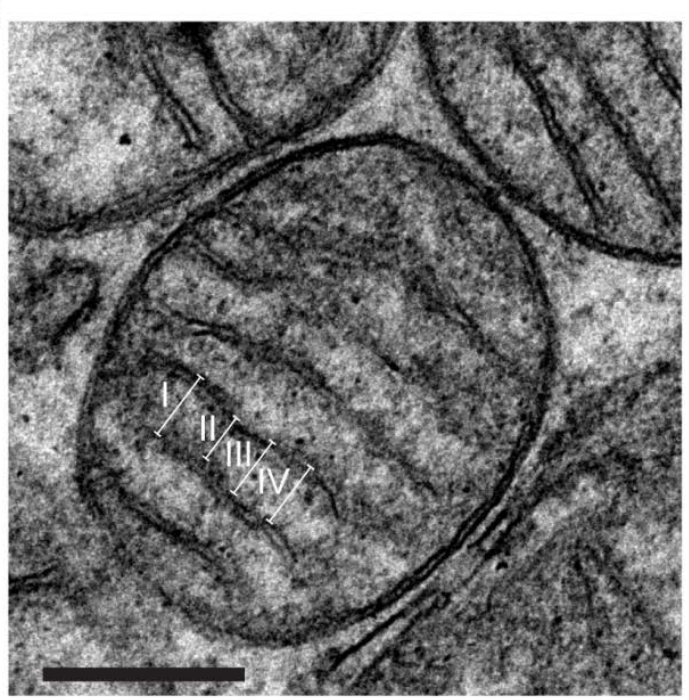

Figure 65: Determination of inter-cristae distance. a) A representative EM micrograph of cryosectioned U2OS cells is shown. Analysis of cristae distance was done on individual mitochondria (dashed lines). b) Multiple distances between each two cristae were measured. The obtained values for the given pair of cristae are $74 \mathrm{~nm}$ (I), $55 \mathrm{~nm}$ (II), $67 \mathrm{~nm}$ (III), and $71 \mathrm{~nm}$ (IV). Scale bar: $300 \mathrm{~nm}$.

Based on the previous calculations of an average total mitochondrial network length of $937 \mu \mathrm{m}$ and an average inter-cristae distance of $80 \mathrm{~nm}$, it can be estimated that a single human cell contains about $1.2 \times 10^{4}$ cristae membranes. Considering that a single genome- 
edited U2OS cell contains about $2.22 \times 10^{6} \mathrm{PHB} 1$ and about $1.07 \times 10^{7} \mathrm{PHB} 2$ molecules, this translates into 185 PHB1 and 892 PHB2 molecules per individual cristae. Previous dual-color STED imaging results suggest that both prohibitins are found in one complex (Figure 55). Further, PHB2 is about 5 times more abundant than PHB1 (Figure 62). Therefore, it is tempting to speculate that the stoichiometry of the human PHB complex between PHB1 and PHB2 is 1 to 5. Assuming a molecular mass of 1 MDa for the human PHB complex (Nijtmans et al., 2000), a 1:5 stoichiometry means that $167 \mathrm{kDa}$ of the complex correspond to PHB1 and $833 \mathrm{kDa}$ to PHB2. Taken the respective MW of both prohibitins into account, each PHB complex would contain about 6 PHB1 (29.8 kDa) and about 25 PHB2 (33.3 kDa) subunits. Because the total copy number of each prohibitin was determined here, this would translate into 3.7-4.3 x 105 PHB complexes per single cell. Together with $1.2 \times 10^{4}$ cristae membranes per cell, this means that about 31-36 single PHB complexes exist per individual cristae membrane. However, these numbers assume a 1:5 stoichiometry between PHB1 and PHB2 and might be interpreted differently as discussed later on.

Taken together, overexpression of PHB1-DK and PHB2-DK in human U2OS cells causes two different aberrant mitochondrial phenotypes. While PHB1-DK overexpression causes a perinuclear accumulation of enlarged mitochondria, PHB2-DK overexpression mainly results in cytoplasmic fluorescent aggregates that are not imported into mitochondria. Both phenotypes are caused by overexpression from a heterologous plasmid promoter and can be prevented by CRISPR-mediated endogenous DK tagging at the C-terminus of the respective prohibitin. Further, overexpression of PHB2-DK together with ESR1 leads to nuclear accumulation of PHB2 in HeLa cells. However, endogenously tagged PHB2-DK HeLa cells showed an exclusive mitochondrial localization of PHB2, which indicates that the nuclear localization of PHB2 is an overexpression-induced artefact. Interestingly, the use of nucleaseresistant donor plasmids lead to a 2-fold increase of homologous recombination frequencies with a maximum observed integration efficiency of about $18 \%$. Despite screening 49 or 74 individual clones, no homozygous PHB1-DK or PHB2-DK knock-in cell lines could be obtained. An impairment of prohibitin function due to homozygous tagging with a fluorescent protein tag cannot be excluded. However, further experiments are required to clarify this issue. Moreover, DNA sequencing of on- and off-target sites in selected knock-in clones revealed the high specificity of each gRNA for the respective target region, because no mutations at unintended genomic sites could be found. 
Intriguingly, STED super-resolution microscopy discovered that prohibitin forms individual clusters and that the distribution of prohibitins in genome-edited cells is highly similar to prohibitin distribution in wildtype cells. Further, dual-color STED imaging revealed a high degree of co-localization between tagged and untagged prohibitins at the nanoscale, which suggests that tagged PHB1-DK and PHB2-DK is incorporated into the PHB complex normally. Interestingly, prohibitins are almost exclusively (85-90\%) localized to the mitochondrial cristae membrane as demonstrated using immunogold EM. The same results were obtained in both genome-edited knock-in cells and wildtype U2OS cells, indicating that the fluorescent protein tag does not affect submitochondrial prohibitin localization. Further, FRAP analysis of prohibitin dynamics in living knock-in cells showed that PHB1-DK and PHB2-DK are exceptionally static proteins with mobile fractions of only 10-20\%. In addition to results on localization and dynamics, this study provides first quantitative data on the number of prohibitins in human cells. Unexpectedly, quantitative western blotting revealed that the amount of PHB2 is about 4-5 times higher than that of PHB1 challenging the view of a previously assumed 1:1 complex stoichiometry. Interestingly, the integration of the obtained data on prohibitin localization, abundance and ultrastructural organization of human mitochondria suggests that an estimated average of about 31-36 PHB complexes resides within individual cristae membranes. In conclusion, CRISPR-mediated endogenous tagging revealed previously inaccessible insights about the localization, dynamics and abundance of human prohibitins. In principle, the presented methods are of general applicability and can be applied to investigate any other 'taggable' mitochondrial protein, which will allow further insights into the dynamic and quantitative organization of this intriguing organelle. 


\section{Discussion}

Fluorescent proteins (FPs) are among the most commonly used tools for fluorescence microscopy investigations of living cells in modern life science laboratories. At the same time, overexpression of FP fusion proteins is a widely shared concern as it leads to a variety of artefacts (Gibson et al., 2013; Rizzo, 2010). Therefore an alternative labelling strategy based on the use of site-specific nucleases for endogenous protein tagging in human cells was established in this thesis. Apart from preventing overexpression-induced artefacts, endogenous tagging has several further advantages. First, the site-specific incorporation of FP cDNA into the genome of a human cell leads to stable and homogeneous expression of fusion proteins resulting in low variation between various assays. Second, endogenous tagging of a protein under its native promoter facilitates long-term imaging of protein homeostasis, dynamics and localization. Third, native protein expression levels would allow the global and local quantification of protein copy numbers in living cells, an approach so far only realized in yeast cells (Huh et al., 2003; Ghaemmaghami et al., 2003; Wu and Pollard, 2005; Puchner et al., 2013). The endogenous tagging method was applied as a novel labelling strategy for super-resolution microscopy and allowed quantitative insights into the localization and dynamics of mitochondrial prohibitins.

\subsection{RESOLFT imaging of endogenously tagged $\beta$-actin}

RESOLFT super-resolution microscopy uses low light intensities and is therefore particularly suited for studies of living cells and tissues (Brakemann et al., 2011; Grotjohann et al., 2011; Grotjohann et al., 2012; Testa et al., 2012; Chmyrov et al., 2013; Lavoie-Cardinal et al., 2014; Testa et al., 2015). Although these studies showed that RESOLFT is a powerful method to visualize dynamic processes in living cells at the nanoscale, however, visualization of the respective protein of interest was achieved via its overexpression as a FP fusion.

The present work showed, for the first time, ZFN-mediated site-specific integration of Dreiklang and rsEGFP2 at the human $\beta$-actin (ACTB) locus (Figure 14). A similar approach was used for successful N-terminal tagging of $\beta$-actin with EGFP in U2OS cells (Fetter et al., 2015). Similar to EGFP, both Dreiklang and rsEGFP2 are monomeric, bright and photostable proteins that are well expressed in mammalian cells (Brakemann et al., 2011; Grotjohann et al., 2012). Therefore it was not unexpected that endogenous tagging would also work using these reversibly switchable fluorescent proteins (RSFPs). However, fusion protein 
overexpression levels can easily be orders of magnitude higher than endogenous protein levels (Figure 39). In general, high fluorophore concentrations often result in quenching, changes in lifetime, and homo FRET (Lakowicz, 2007). Further, environmental factors can slow down photoswitching kinetics of RSFPs (Kao et al., 2012). Hence, lower fluorophore expression levels were expected to be beneficial. On the other hand, bleaching of fewer available RSFPs and a reduced number of fluorescent photons obtained from the remaining fluorophores might not have been sufficient to obtain a super resolved image with good contrast and signal-to-noise ratio (Hell, 2009).

We chose $\beta$-actin as a positive control for endogenous tagging because of its high cellular abundance of about $5 \times 10^{8}$ molecules per cell (Lodish, 2008). In practice this led to the first demonstration that RESOLFT might work on endogenously tagged DK- $\beta$-actin and rsEGFP2$\beta$-actin knock-in cells (Figure 15 and 16). However, $\beta$-actin is present either as globular (G)actin or as a subunit of filamentous (F)-actin (Lodish et al., 2008). Free RSFP-tagged G-actin causes a highly diffuse fluorescent signal within most parts of the respective cell. Therefore, RESOLFT imaging was performed on thick stress fibers at the cell periphery where the Factin signal was high. Whereas each F-actin microfilament measures about $6 \mathrm{~nm}$ in diameter (Fuchs and Cleveland, 1998), stress fiber diameter ranges from 60-180 nm as each fiber is composed of 10-30 F-actin filaments (Tojkander et al., 2012). Thus, a high background signal caused by free RSFP-G-actin and RESOLFT analysis of thick stress fibers led to a merely enhanced resolution compared to confocal imaging. Indirect F-actin labeling strategies based on Lifeact (Riedl et al., 2008), SiR-actin (Lukinavicius et al., 2014), or Actin-Chromobody ${ }^{\circledR}$ (ChromoTek, Munich, Germany) will achieve a higher imaging contrast and are therefore more suitable for live cell super-resolution imaging studies than endogenously tagged $\beta$-actin cells.

In the presented part of this work, ZFNs were used as tools for site-specific RSFP integration at the ACTB locus. Although ZFN design, engineering and assembly simplified over the last years, ZFN generation generally requires constructing and screening large combinatorial libraries (Urnov et al., 2010; Sander et al., 2011). Therefore widespread adoption and largescale use of ZFN technology are restricted to a few expert labs. Customized ZFNs are available commercially, but their cost restricted the scale of research projects that can be realized. Similar to ZFNs are transcription activator-like effector nucleases (TALENs) (Bogdanove and Voytas, 2011). TALENs contain multiple DNA binding domains and each domain recognizes one nucleotide. The involved protein-DNA interactions are less complex compared to the interaction between each ZFNs domain and its target tri-nucleotides. Thus 
TALEN design and assembly are more straightforward than ZFN generation. However, for each target site a new TALEN protein has to be assembled in a time-consuming fashion. Therefore we decided to establish our own genome editing pipeline based on the recently introduced clustered regularly interspaced palindromic repeats (CRISPR) system that offers several advantages over both ZFNs and TALENs (Jinek et al., 2012; Cong et al., 2013; Mali et al., 2013).

\subsection{CRISPR-based endogenous protein tagging}

In order to independently and cost-efficiently generate a large amount of endogenously tagged cell lines, we chose to establish a general workflow based on the CRISPR system. CRISPR is a simple, efficient and inexpensive genome engineering tool based on the versatile RNA-guided nuclease Cas9. Target recognition by Cas9 is based on DNA-RNA interactions and therefore does not require re-engineering a new protein for each target site. Cas9 is simply programmed by a guide RNA (gRNA) for targeting nearly any DNA sequence in the human genome. Plasmid construction is based on oligo cloning and therefore simple and straightforward.

\subsubsection{Choice of cell lines}

CRISPR-based genome engineering was done in human U2OS or HeLa cells. These cell lines are of human origin, easily cultivated and suitable hosts for co-transfection of large and/or multiple plasmids such as Cas9/gRNA and, if required, donor plasmids. Further, U2OS and HeLa cells are immortalized and grow indefinitely, an important feature for cell expansion after single cell sorting. Also both cell lines exhibit epithelial adherent morphology and are frequently used model systems for super-resolution imaging and studies of human mitochondrial biology. A disadvantage of using cancer cells is their genetic instability and their aneuploidy (Janssen and Medema, 2013). The most frequently used cells of this study were U2OS cells, a cell line that is chromosomally highly altered and contains chromosome counts in the hypertriploid range (Ponten and Saksela, 1967; Ben-Shoshan et al., 2014). This might pose challenges when homozygous knockout or knockin cells are to be generated as more alleles per target gene have to be modified. In fact, while screening as few as two clones was sufficient to obtain a homozygous ZYX-rsEGFP2 cell line (Figure 30), none of the 74 screened PHB2-DK clones was homozygous for the transgene (Figure 47). While U2OS cells contain two copies of the ZYX gene, the PHB2 gene is present in five copies (Halling-Brown et al., 2011) and multiple rounds of genome editing might be required in order to target every 
allele (Lazarou et al., 2015). Haploid human cancer cell lines like KBM7 or HAP1 (Carette et al., 2011; Burckstummer et al., 2013) might reduce the effort required to generate a homozygous mutant, but their round morphology and a high nucleo-cytoplasmic ratio poses difficulties for imaging applications.

An alternative to cancer cell lines are primary cultures derived from normal animal tissue that retain many of the characteristics of the cell in vivo. However, primary cells have a limited lifespan and can be maintained in vitro only for a restricted time (Hayflick and Moorhead, 1961; Hayflick, 1965). As genome edited monoclonal cell lines are established via isolation and expansion of a single cell, replicative senescence occurring in untransformed cell lines would make this selection step impossible.

In conclusion, the advantages of using human cancer cell lines over primary cells for CRISPR-based gene editing is that they are easy to transfect and their indefinite growth potential allows the isolation of monoclonal cell lines after single cell sorting. Chromosomal aberrations in cancer cells and high gene copy numbers together with potentially inaccessible loci might slow down or even inhibit the generation of homozygous mutants. However, this might depend on the gene to be modified as CRISPR has been used recently to sequentially knockout five genes in HeLa cells (Lazarou et al., 2015).

\subsubsection{Genomic target site selection}

Target DNA sequences for gRNAs must contain a protospacer adjacent motif (PAM)-site of the sequence 5'-NGG-3' immediately downstream of the gRNA binding site (Jinek et al., 2012; Cong et al., 2013; Mali et al., 2013). Localization of PAM sites for knockouts is usually not difficult as many possible target sites exist within one of the first few exons. The choice of target sites, however, for knock-ins via HR is dictated by the desired location of insertion and thus more constrained. In general, selection of gRNA target sites within the human genome was done using the CRISPR Design Tool (Hsu et al., 2013). The algorithm scans an input sequence for possible CRISPR targeting sites with the sequence 5'-N20-NGG-3' and potential off-target matches are analyzed throughout the selected genome. Off-target sites associated with potential gRNAs are computed by taking into account: 1) the total number mismatches between on- and off-target sites, 2) the position of the mismatch relative to the PAM site and 3) the mean pairwise distance between mismatches. The algorithm lists all possible gRNA designs and predicted number and sequence of associated off-target sites. However, off-target modifications might also occur in sites that are not included in the potential candidate list. ChIP-seq identified a wide range of $>100$ Cas 9 off-target binding sites 
for certain gRNAs and targeted sequencing showed that Cas9 modifies some but not all bound off-target sites (Kuscu et al., 2014). Ideally, whole genome sequencing of every generated cell line should be performed to verify the absence of unwanted modifications at off-target sites. However, this is approach is not straightforward as it is costly and requires expertise in data analysis.

While multiple Cas9 mutants with improved specificities exist, the use of these mutants poses further constraints on target site selection and often leads to a reduced on-target activity (Hsu et al., 2014). However, the main goal of this study was to generate endogenously tagged human knockin cell lines. Therefore, the choice of target sites is already restricted to the start or stop codon region in order to achieve high HR frequencies. Moreover, transgene integration via $H R$ requires highly efficient on-target mutagenesis as $H R$ frequencies are much lower than initial DSB induction rates. Therefore, instead of relying on low activity Cas 9 mutants, we decided to use wildtype Cas9 that was reported to exhibit on-target efficiencies of up to $77 \%$ (Friedland et al., 2013). As discussed below, this approach was successfully realized and mutations at predicted off-target sites were not detected.

\subsubsection{In vivo vs in vitro functional gRNA validation}

Functional validation of initially generated gRNAs was done using the T7 Endonuclease I (T7EI) assay or an in vitro approach. T7El is a mismatch-specific endonuclease and cleaves heteroduplex DNA formed after melting and hybridizing mutant and wildtype alleles (Mashal et al., 1995). The advantage of using the T7El assay is that once a functional gRNA is found, this functionality reflects the in vivo gRNA activity. However, the T7El assay suffers from low sensitivity as only mutation rates of above $5 \%$ are detected (Vouillot et al. 2015) and takes 34 days to complete. The in vitro functional testing of gRNAs takes only about 1-2 days, but it is not guaranteed that the tested gRNAs will also be functional in vivo as chromatin structure and DNA modifications affect Cas9-gRNA targeting (Kuscu et al., 2014; Wu et al., 2014b). Another strategy to quantify mutation rates after gRNA delivery is Sanger or next-generation sequencing of a representative panel of sub-cloned PCR products. For routing gRNA testing both approaches are expensive and require several days to complete. Further, highresolution melting curve analysis has been used to identify mutations (Dahlem et al., 2012) but requires the development of a specialized quantitative PCR assay. The main objective of this work was using CRISPR for endogenous FP tagging. HR not only depends on a gRNAinduced DSB but also on DNA methylation status and chromatin structure of the targeted locus (Liang and Jasin, 1995; Ramdas and Muniyappa, 1995) as well as the specific 
sequences that undergo HR (Smith, 1994). Thus instead of testing multiple gRNAs for mutagenic activity, the Cas9-gRNA pairs were directly validated according to their ability to mediate FP integration at the locus of interest.

In conclusion, the choice of the gRNA validation method depends on the intended application. In general, it is recommended to generate multiple gRNAs targeting the same genomic region at multiple sites. For knockout studies, each gRNA should be functionally validated using the T7El assay, because it provides information about gRNA functionality in vivo. For knock-in projects, gRNAs should be tested together with the respective donor plasmid for their capability to induce a DSB that can be repaired efficiently via HR.

\subsubsection{Generation of donor plasmids}

Repair templates for targeted genomic insertions of large transgenes, such as fluorescent protein DNA, require the use of donor plasmids that contain homology arms flanking the transgene (Smithies et al., 1985; Thomas and Capecchi, 1986). Length of each homology arm should be at least 500 bp (Hasty et al., 1991). A streamlined procedure for donor plasmid design and construction exist (Wu et al., 2008). This method is based on recombinationbased cloning methods consisting of three steps and it takes about two weeks to construct a donor plasmid. Methods for faster generation of donor plasmids were established here using two-step cloning or one-step Gibson assembly (Gibson et al., 2009) for joining multiple fragments. Gibson assembly is preferred as a donor plasmid can be efficiently generated in one step and obtained in a sequence-verified manner within 4 days starting with ready-toassemble fragments. Moreover, Gibson assembly is a 'scarless' cloning method as no restriction site remains between adjacent fragments, an important feature when multiple DNA fragments have to be ligated that together reconstitute a single exon.

An important point to mention is that Cas9 will a) continue to modify the allele after donor plasmid insertion and/or b) degrade the donor plasmid before insertion as long as the gRNA target and PAM site remain intact. This could lead to unwanted mutations after transgene insertion at the gRNA target site and/or reduce the efficiency of transgene integration. Therefore, it is advisable to generate a repair template that contains (silent) mutations in the gRNA and/or PAM site that will avoid secondary mutations after insertion. Further, transgene incorporation frequencies inversely correlate with the distance from the DSB (Elliott et al., 1998). Transgene insertion rates drop about four fold at a distance of $100 \mathrm{bp}$ away from the DSB and beyond a distance of 200 bp or more away, drug selection markers may be required 
(Elliott et al., 1998; Ran et al., 2013b). Therefore, gRNA was done such that the fluorescent protein insertion site was not more than 100 bp away from the DSB.

\subsubsection{Clone isolation via FACS and characterization of monoclonal cell lines}

After successful integration of a fluorescent reporter gene, selection of single modified cells was done using fluorescence activated cell sorting (FACS) to obtain monoclonal cell lines. Major advantages of FACS are its rapid and quantitative analysis of multiple parameters simultaneously in a highly sensitive and reproducible manner (De Rosa et al., 2001). Further, FACS sorters have a low error rate and can detect and purify rare subsets of cells as low as $0.01 \%$. However, maintaining sterility when isolating single cells via FACS might pose problems and cell damage due to hydrodynamic stress causes slow recovery or even apoptosis of sorted cells (Mollet et al., 2007). Alternative methods for single cell isolation include limiting dilution protocols that are cost-effective but tedious and impractical when many clones are to be isolated. Further, cloning rings for harvesting individual colonies can be employed after cells were seeded sparsely in $10 \mathrm{~cm}$ dishes and allowed to expand to form single colonies. Here the throughput might be higher but there is no guarantee that the colony originated from a single cell.

Analysis of the monoclonal cell lines was streamlined and involved genotyping via PCR, assessment of fusion protein expression via western blotting (WB) and on-/off-target site analysis using Sanger sequencing. Importantly, genotyping via PCR must be done with a primer pair in which at least one of the two primers anneals outside of the homology arms. This ensures the locus-specific analysis of the transgene integration event. For instance, random integration of the donor plasmid would lead to presence of homology arm DNA at an off-target locus. In that case, use of two primers that anneal within the homology arms (in-in PCR) would lead to the wrong interpretation that such a clone was successfully modified.

Taken together, the established workflow allows for efficient endogenous protein tagging with fluorescent reporters using the CRISPR system and wildtype Cas9 in human cancer cell lines. The entire protocol, from planning to obtain a characterized cell line, takes about six weeks. This time could be reduced by using a cell line with a faster growth rate, because recovery of U2OS cells after single cell sorting takes about 2-3 three weeks. Further, an efficient strategy for selecting a homozygous knock-in clone at the initial FACS sorting step is lacking. To select clones with e.g. at least two or three knock-in alleles a double or triple antibiotic selection strategy could be employed to enrich for modified clones before FACS selection. However, further experiments are needed to test the potential of this approach. 


\subsection{Endogenous tagging for live cell super-resolution microscopy}

The previously established workflow for CRISPR-mediated endogenous tagging was successfully applied to generate 24 human knock-in cells (Table 15). A selection of three endogenously tagged cell lines was used for in-depth characterization and investigation using flow cytometry, confocal and RESOLFT super-resolution microscopy.

\subsubsection{Increasing integration frequencies and survival rates after single cell sorting} The three proteins vimentin (VIM), zyxin (ZYX) and high mobility group protein A1 (HMGA1) were successfully targeted for C-terminal integration of the reversible switchable fluorescent protein rsEGFP2 (Table 1). Single cell sorting via FACS allowed the selection of targeted fluorescent clones even at low homologous recombination (HR) frequencies below $1 \%$. However, endogenous protein tagging with widely used affinity tags such as FLAG, c-myc or streptavidin (Terpe, 2003) would be attractive for protein purification studies. Those tags are non-fluorescent and maximum homologous recombination (HR) rates are required to decrease the number of clones that must be screened. Because a DNA double strand-break is either repaired via HR or non-homologous end-joining (NHEJ), inhibition of the NHEJ pathway leads to knock-in frequencies of 50-66 \% (Chu et al., 2015). Further, HR occurs only during $S$ and $G 2$ phase and cell cycle synchronization lead to an increase of HR frequencies up to $38 \%$ compared to non-synchronized cells (Lin et al., 2014). A combination of both approaches, NHEJ inhibition and cell synchronization, might even result in higher knock-in frequencies, but this remains to be experimentally verified.

While selection of fluorescent knock-in cells via FACS is straightforward, typical recovery rates of U2OS cells after single cell sorting were only 10-20 \%. Low survival rates are due to cell damage caused by hydrodynamic stress during sorting that can result in apoptosis of sorted cells (Mollet et al., 2007). Switching to a larger nozzle or decreasing the flow rate during sorting might increase viability (Tol et al., 2008). Further, recovery rates can be increased by supplementing the growth medium with antioxidants such as $\alpha$-thioglycerol or bathocuprione disulphonate (Brielmeier et al., 1998) or higher concentrations of fetal bovine serum (FBS).

\subsubsection{Gene editing of HMGA1, VIM and ZYX using CRISPR is highly specific}

DNA sequencing of on-target sites showed that Cas9-induced DSBs mainly resulted in small deletions with a length of 1, 2, 3 or 11 bp or small insertions of 1 bp (Figure 33-35). This is in line with previous studies showing that the most common insertions/deletions (indels) are 1 
bp insertions and 1-3 bp deletions (Cradick et al., 2013). Importantly, mutagenesis of the terminal exon of the ZYX gene was prevented by introduction of silent mutations within the gRNA binding site that did not lead to alterations in the protein sequence. It should be noted that the observed mutations were located within the 3'-UTR of the VIM or HMGA1 gene, respectively. This region contains important elements for regulation of eukaryotic gene expression (Barrett et al., 2012) and even single nucleotide changes within the 3'-UTR can greatly reduce mRNA translation (Clop et al., 2006). However, expression levels of both affected proteins, VIM and HMGA1, were comparable to expression levels in wildtype cells (Figure 32). Hence the observed mutations do not seem to influence any major posttranscriptional regulation mechanism.

DNA sequencing revealed no mutagenesis of the computationally predicted off-target sites in any of the monoclonal knock-in cell lines generated (Figure 36-38). This was expected, because the number of mismatches between the respective gRNA and each analyzed offtarget DNA site was 3-4 bp (Table 2). In general more than three mismatches between the potential off-target sites and the complementary gRNA are not tolerated (Hsu et al., 2013; Ran et al., 2013b). Further, most mismatches were positioned within the highly mismatchintolerant 12 nucleotide seed region on the 3'-end of the respective gRNA (Hsu et al., 2013; Ran et al., 2013b). However, only two potential off-target sites per gRNA could be analyzed and off-target modifications might also occur in sites that are not included in the potential candidate list (Wang et al., 2015). Further, some gRNAs can tolerate up to five mismatches with unwanted target sites (Fu et al., 2013). Technically, whole genome sequencing of every generated cell line is done, but this is a highly impractical approach, because it is timeconsuming, expensive and requires significant expertise in sequencing data analysis. Practically, the here presented approach of generating multiple, independently targeted clones using two different gRNAs is advisable. While this strategy does not reduce off-target mutagenesis itself, it can be assumed that on-target modification caused the observed phenotype, if the same phenotype is associated with several gRNAs (Ran et al., 2013b).

\subsubsection{Endogenous tagging prevents overexpression artefacts}

FACS and microscopic analysis of rSEGFP2 knock-in cells demonstrated the advantage of endogenous tagging over ectopic expression from heterologous promoters. Strikingly, fusion protein expression levels spanned three orders of magnitude between $10^{2}$ and $10^{5}$ arbitrary units independent of the expressed protein (Figure 39). While about 25\% of the cells were transfected, an individual cell must have taken up various plasmid copies. In fact, previous 
studies showed that plasmid transfection is highly heterogeneous on the levels of cellular and nuclear uptake to final transgene expression (Cohen et al., 2009). Compared to ectopic expression, endogenous expression levels were characteristic for the respective protein, indicating that gene expression is controlled by endogenous promoters and regulatory elements. Interestingly, FACS revealed the physiological variation in protein abundances on a single cell level. Although individual cells within each monoclonal rsEGFP2 knock-in cell were genetically identical, they exhibited substantial phenotypic heterogeneity. This variation is caused by differences in cell size and cell cycle states, but can also arise from stochasticity in gene expression. The resulting cellular heterogeneity can be essential for many biological processes (Kaern et al., 2005), but its investigation was so far limited to endogenously tagged bacterial (Elowitz et al., 2002) and yeast cells (Di Talia et al., 2007). Therefore, CRISPRmediated endogenous tagging can be applied to extend studies on cellular heterogeneity to human cells.

Microscopic investigation of U2OS cells after plasmid transfection demonstrated a multitude of artefacts including mislocalization and aggregation of fusion proteins as well as changes in cell shape (Figure 40). High expression rates often result in protein aggregation due to an overload of the protein folding machinery (Kober et al., 2012; Halff et al., 2014), mislocalizations due to deregulated protein trafficking (Hung and Link, 2011) and cell morphology changes especially when cytoskeletal proteins are overexpressed (Mendez et al., 2010). However, endogenously tagged cells exhibited protein distributions and cell morphologies comparable to wildtype cells. This demonstrates that the previously observed artefacts were caused by artificially high fusion protein expression levels that disturbed cellular homeostasis, but not by the fluorescent protein tag itself.

\subsubsection{RESOLFT allows nanoscale imaging at endogenous expression levels}

RESOLFT super-resolution imaging was successfully applied to reveal the nanoscale distribution and dynamics of endogenously rsEGFP2-tagged cell lines. Compared to conventional imaging, RESOLFT could readily image VIM-rSEGFP2 cells at a resolution down to $40 \mathrm{~nm}$ (Figure 41). Further, super-resolution movies showing highly motile vimentin networks on a time-scale of seconds and minutes could be recorded. Previous RESOLFT imaging studies on VIM-rsEGFP2 showed a less mobile intermediate filament network, suggesting that overexpression might negatively impact vimentin dynamics (Grotjohann et al., 2012). RESOLFT on HMG-I-rSEGFP2 could visualize individual protein clusters in the nucleus of an endogenously tagged human cell for the first time (Figure 42). 3D super- 
resolution RESOLFT microscopy (Testa et al., 2012) could further improve image resolution as HMG-I-rsEGFP2 is three-dimensionally distributed over the nucleus. Finally, RESOLFT could distinguish individual zyxin fibers as well as zyxin dynamics homozygous knock-in cells. Compared to confocal imaging, RESOLFT was able to distinguish individual zyxin nanoclusters previously only observed using PALM imaging (Betzig et al., 2006; Shroff et al., 2007). Further, these studies required up to 30 minutes to obtain a single image (Shroff et al., 2007) whereas RESOLFT imaging took about 3 minutes per image. RESOLFT image acquisition time can be further reduced 4-fold via doubling the pixel length. This strategy would result in lower phototoxicity, but also in a 2-fold lower lateral resolution. Additionally, the recording speed could be further accelerated and large fields of view $\left(100 \times 100 \mu m^{2}\right)$ recorded using massively parallelized scanning (Chmyrov et al., 2013).

In conclusion, CRISPR-mediated genome engineering was demonstrated to generate heterozygous and homozygous human cell lines expressing rsEGFP2 fusion proteins. For the first time, this labelling strategy was adopted for RESOLFT super-resolution imaging. Ideally, homozygous knockin cells are obtained, as they will allow quantitative analysis of endogenous protein numbers and localizations on the nanoscale in the future. The approach is versatile since instead of rsEGFP2 incorporation, also other suitable (fluorescent) tags could be used.

\subsection{Quantitative analysis of mitochondrial prohibitins in human cells}

Besides their role in cristae morphogenesis, prohibitins have been implicated in various processes such as transcriptional regulation, cellular signaling and others (Merkwirth and Langer, 2008). Next to mitochondria, prohibitins were found to localize to the nucleus (Fusaro et al., 2003; Kurtev et al., 2004; Kasashima et al., 2006) and the plasma membrane (Kolonin et al., 2004; Sharma and Qadri, 2004) in various cell types and model systems. Therefore, it was suggested that the functional diversity of prohibitins correlates with their diverse localizations to different cellular compartments. Interestingly, it was proposed that PHB2 translocates from mitochondria to the nucleus in presence of estradiol and estrogen receptor (Kasashima et al., 2006). However, complementation assays in mouse embryonic fibroblasts (MEFs) strongly support the fact that cellular homeostasis solely depends on the mitochondrial localization of prohibitins (Merkwirth et al., 2008). Further, many studies were based on prohibitin overexpression that potentially leads to protein malfunction and mistargeting to diverse cellular compartments (Fusaro et al., 2003; Kurtev et al., 2004, Sun et al., 2004; Kasashima et al., 2006). 
To investigate the subcellular distribution of PHB1 and PHB2 in human U2OS cells at endogenous expression levels, we set out to employ CRISPR-based genome editing for genomic tagging of each prohibitin. For the first time, this approach allowed the comprehensive analysis of prohibitin localization, dynamics and abundance in human cells using a variety of methods.

\subsubsection{Overexpression of PHB1-DK or PHB2-DK induces aberrant mitochondria}

Transient overexpression of Dreiklang (DK)-tagged prohibitin 1 (PHB1) or prohibitin 2 (PHB2) resulted in two different aberrant mitochondrial morphologies. The majority of PHB1-DK transfected cells displayed perinuclear clusters of enlarged mitochondria (Figure 43). Interestingly, a similar phenotype was observed upon enforced expression of the outer mitochondrial membrane (OMM) fusion protein Mfn1, which leads to mitochondrial aggregation by stimulating OM fusion events (Santel et al., 2003). Thus PHB1 overexpression might promote OM fusion indirectly, which would be in agreement with the observed inhibitory effect that PHB1 exerts on mitochondrial fission events (Wang et al., 2014b).

PHB2 overexpression causes mitochondrial fragmentation or accumulation of cytoplasmic protein aggregates (Figure 43). Interestingly, fragmented mitochondria were observed upon PHB2 knockout as well as PHB2 overexpression (Merkwirth et al., 2008; Kowno et al., 2014), suggesting that any imbalance in PHB2 expression levels leads to fragmented mitochondria. Accumulation of cytoplasmic PHB2-DK aggregates might be attributed to an overloaded cytoplasmic chaperone system dedicated to mitochondrial precursor protein stabilization prior to import (Young et al., 2003). Strikingly, cytoplasmic aggregates where never observed upon ectopic expression of PHB1-DK, but only detected when PHB2-DK was overexpressed. Using membrane topology prediction algorithms, it was proposed that PHB2 contains a transmembrane helix, while PHB1 is expected to be membrane associated (Back et al., 2002). Because of the transmembrane helix, PHB2 is more hydrophobic and the intrinsic selfaggregation properties of PHB2 might be higher than for PHB1. However, experimental evidence for this hypothesis is lacking and structural studies are needed for clarification.

\subsubsection{Endogenously tagged PHB1-DK and PHB2-DK clones are heterozygous and exhibit fusion protein expression variability}

In order to prevent artefacts induced by overexpression of PHB1-DK or PHB2-DK, the previously established CRISPR-based workflow for endogenous tagging was applied. While knock-in efficiencies reached up to 17.7\% (Figure 46) and between 49 and 74 individual clones were screened, no homozygous PHB1-DK or PHB2-DK knock-in cell was found 
(Figure 47). It cannot be excluded that homozygous tagging with a fluorescent reporter would render the respective prohibitin non-functional and cause a lethal phenotype. However, previous studies in yeast and mammalian cells suggest that PHB1-GFP and PHB2-GFP are functional (Birner et al., 2003; Kasashima et al., 2006; Kowno et al., 2014). Thus, absence of homozygous clones might be attributed to the insufficient targeting of all PHB1 or PHB2 gene copies. In fact, analysis of copy number variations in U2OS cells revealed that four copies of the PHB1 gene exist in these cells, while five PHB2 gene copies are present (Halling-Brown et al., 2011). Therefore, a homozygous modification could be prevented, if only a fraction of all gene copies was accessible for site-specific gene editing. This is in agreement with the observation that at least one gene copy of each PHB1 and PHB2 displayed a wildtype DNA sequence, suggesting that it was not modified by Cas9 (Figure 51 and 52). However, further experiments including multiple rounds of transfection with nuclease/donor plasmids and single clone isolation are needed to test whether homozygous tagging of PHB1 or PHB2 is possible.

Expression level analysis of four PHB1-DK and four PHB2-DK clones revealed that total PHB1 levels varied substantially between different clones, while PHB2 levels were similar among most clones (Figure 48). The used gRNAs were targeted towards the 3'-UTR of the respective gene, a region that contains crucial elements for the regulation of gene expression levels (Barrett et al., 2012). While the 3'-UTR of PHB2 is not characterized, it has been reported that PHB1 mRNA contains a long 3'-UTR comprising a microRNA response element to control PHB1 protein levels (Jupe et al., 1996; Wang et al., 2014b). CRISPR-induced mutagenesis of PHB1 might result in 3'-UTR modifications that vary among clones and have a different impact on PHB1 expression levels. Alternatively, gRNA-specific off-target sites at unintended genomic regions might alter PHB1 protein levels.

\subsubsection{Endogenously tagged PHBs are found in mitochondria}

This thesis demonstrated that endogenous tagging of PHB1 and PHB2 with DK leads to mitochondrial localization of both PHB1-DK and PHB2-DK (Figure 49). Importantly, these knock-in cell lines exhibited a wildtype mitochondrial morphology compared to forced overexpression conditions that induce an aberrant mitochondrial phenotype. This shows that not the fluorescent protein tag itself, but rather the non-physiological expression rates of the respective protein caused aberrant mitochondria. Further, the central claim of the 'gene dosage balance' hypothesis is that perturbation of the stoichiometric balance in protein 
complexes can be a source of abnormal phenotypes (Birchler and Veitia, 2012). This hypothesis could be confirmed experimentally for the PHB complex in the present study. Interestingly, nuclear localization of PHB2-DK in presence of estrogen receptor a (ESR1) and $17 \beta$-estradiol (E2) could be demonstrated in HeLa cells (Figure 61) as shown before (Kasashima et al., 2006). However, the nuclear accumulation of PHB2-DK was no general phenomenon as it was only observed in a fraction of cells co-expressing PHB2-DK and ESR1. In fact, nuclear localization of PHB2 might be attributed to very high PHB2-DK overexpression levels achieved only in a fraction cells. This was confirmed by ESR1 overexpression in endogenously tagged HeLa-PHB2-DK cells where PHB2-DK is solely localized in mitochondria (Figure 61). As previously observed for PHB2-DK overexpression in U2OS cells, most PHB2-DK is not imported into the mitochondrion but rather forms cytoplasmic aggregates. Under such conditions, a combination of unfolding and aggregation might generate an artefactual nuclear localization signal (Gibson et al., 2013). Further, E2 was shown to be toxic and to induce apoptosis in ESR1-expressing HeLa cells (Kushner et al., 1990; Zhang and Shapiro, 2000). It can be concluded, that PHB2 nuclear mistargeting in HeLa cells is an overexpression artefact only observed under non-physiological conditions with artificially high PHB2 expression levels.

\subsubsection{Prohibitins form immobile clusters in the inner mitochondrial membrane}

Previous live cell confocal imaging of endogenous PHB1-DK and PHB2-DK showed that prohibitins localize to mitochondria in human cells. STED super-resolution microscopy on fixed cells revealed distinct clusters of PHB1-DK and PHB2-DK in genome-edited cells (Figure 52). Similarly, wildtype cells exhibited distinct PHB1 and PHB2 clusters suggesting that clustering is neither a tag-induced artifact or hindered by the C-terminal tag (Figure 53). The average diameter of individual PHB1 or PHB2 clusters, without decorating antibodies, was estimated to be between 45 and $50 \mathrm{~nm}$. Further, prohibitin clustering was not always uniform throughout a mitochondrial tubule and prohibitin 'hotspots' were observed. Thus, the mitochondrial IM is not homogenous for prohibitin distribution and prohibitin clusters exist within a range of sizes. This is in contrary to other IM proteins such as Mic60 that display a regularly spaced array of clusters within the mitochondrial IM (Jans et al., 2013). However, whether there is a connection between the heterogeneous distributions of prohibitins and their function remains to be investigated. Dual-color STED imaging demonstrated that both PHB1DK and PHB2-DK colocalize with the respective untagged version of both PHB1 and PHB2 (Figure 54 and 55). Interestingly, the distribution of both tagged and untagged PHB1 or PHB2 
were similar, suggesting that antibody staining of the tagged prohibitin is representative for the distribution of both tagged and untagged prohibitin. Importantly, the similar localization suggests that tagged prohibitin is successfully incorporated into the PHB complex.

Immunogold electron microscopy (EM) demonstrated that about $80-90 \%$ of both PHB1 and PHB2 are localized to the CM with only a small pool of $10-20 \%$ localized within the IBM (Figure 56 and 57). Similarly, immunogold EM studies showed that OPA1 is mostly localized to cristae with only a small fraction found within the inner boundary membrane (IBM) (Griparic et al., 2004). OPA1 mediates IMM fusion and a selective loss of long OPA1 isoforms has been observed upon prohibitin depletion in murine cells (Merkwirth et al., 2008). While prohibitins were suggested to regulate OPA1 processing only indirectly (Merkwirth and Langer, 2009), a similar submitochondrial localization of prohibitins and OPA1 might be required for optimal OPA1 processing. Moreover, it has been discussed whether prohibitins are involved in establishing membrane curvatures crucial for the architecture of cristae junctions (CJ) (Zick et al., 2009). A direct role for prohibitins in the establishment for CJs seems unlikely considering that the majority of both PHB1 and PHB2 are localized within the CM. However, PHBs might have an indirect implication via controlled processing of OPA1 which itself is able to induce CJ tightening (Cipolat et al., 2006; Frezza et al., 2006).

A small fraction of $10-20 \%$ of both PHB1 and PHB2 was found to localize to the IBM (Figure 56 and 57). These fractions could simply correspond to newly imported prohibitins under steady state conditions. On the other hand, IBM-enriched prohibitins might also play an important role for processes in the mitochondrial outer membrane (OMM). Interestingly, yeast cells lacking Mmm1, Mdm10 and Mdm12 critically depend on PHBs for survival (Berger and Yaffe, 1998) and these three proteins were shown to be subunits of the ER-mitochondria encounter structure (ERMES), which tethers mitochondria to the ER (Kornmann et al., 2009). Mammalian cells lack ERMES and Mfn2 has been proposed to form a physical ERmitochondria tether (de Brito and Scorrano, 2008; Schneeberger et al., 2013). Mfn2 interacts with SLP2, which binds cardiolipin and SLP2 was proposed to recruit both PHB1 and PHB2 to form cardiolipin-enriched microdomains (Hajek et al., 2007; Da Cruz et al., 2008; Christie et al., 2011). Therefore, it is tempting to speculate that IBM-localized PHBs were recruited via SLP-2 to indirectly modulate the function of Mfn2, thereby influencing mitochondrial fusion and/or contact sites. However, experimental evidence for this hypothesis is lacking and future studies are needed to clarify the potential role of IBM-localized prohibitins.

Fluorescence recovery after photobleaching (FRAP) demonstrated that both PHB1-DK and PHB2-DK were highly immobile (Figure 58). Previous FRAP studies on mitochondrial inner 
membrane proteins found an immobile fraction of about $40 \%$ for the $\mathrm{Y}$-subunit of complex $\mathrm{V}$ and about 50\% for subunit Cox8a of complex IV (Sukhorukov et al., 2010). Those proteins appear to be almost homogenously distributed between IBM and CM (Vogel et al., 2006) and it was suggested that the immobile fraction of $y$-subunit and Cox8a reflects the cristaelocalized fraction of these molecules (Sukhorukov et al., 2010). This is in agreement with the mobility and localization data obtained for both PHB1-DK and PHB2-DK. About $80-90 \%$ of both proteins are enriched in the cristae membrane rendering the same fractions of PHB1-DK and PHB2-DK essentially immobile within the cristae membrane.

\subsection{5. $\mathrm{PHB2}$ is more abundant than PHB1}

Quantitative western blotting demonstrated that a single genome-edited or wildtype U2OS cell contains about 4-5 times more PHB2 than PHB1 (Figure 62 and 63). Assuming a suggested heterodimeric 1:1 stoichiometry between PHB1 and PHB2 (Back et al., 2002), this would mean that about $60-80 \%$ of PHB2 do not participate in PHB complex formation. It is conceivable that such 'free' PHB2 functions independently of PHB1 in other mitochondrial protein complexes. However, dual-color STED imaging showed that both tagged and untagged prohibitins are similarly distributed and no subcomplexes comprising individual PHB1 or PHB2 could be found (Figure 54 and 55). Based on these observations, a 1:5 stoichiometry between $\sim 6$ PHB1 and $\sim 25$ PHB2 subunits for the human PHB complex could be assumed. Intriguingly, this is in contrary to previous investigations that suggested the 1:1 stoichiometry for the yeast PHB complex and found $\sim 14$ copies (Back et al., 2002) or $\sim 18$ copies (Tatsuta et al., 2005) per prohibitin subunit. However, the insights of those studies are based on in vitro structural analysis using crosslinking mass spectrometry and single particle EM of purified yeast prohibitins. Further, the copy number of each prohibitin was extrapolated from the observation that the purified yeast PHB complex runs at an apparent molecular mass of 1-1.4 MDa in a blue-native gel without actually measuring the subunit concentration (Back et al., 2002; Tatsuta et al., 2005). Therefore, it is conceivable that the investigated yeast PHB complex represents the stable core structure that can be purified while a different structural organization of the complex exists in vivo. Further, comparable data for other model systems than yeast do not exist. Therefore, additional structural studies on both yeast and mammalian PHB complexes are required to get a more comprehensive insight into their composition and organization. 


\subsection{Conclusions and outlook}

This work presents the establishment of an efficient workflow for CRISPR-Cas9-mediated generation of human knock-in cell lines that express a fluorescent protein (FP) from a genomic locus. Importantly, site-specific endogenous protein tagging leads to (almost) native fusion protein expression levels, which in turn could prevent artefacts commonly associated with overexpression. This shows that artificially high protein expression levels, but not FP tagging itself, cause a disturbed cellular homeostasis. RESOLFT super-resolution imaging of endogenously tagged cells enabled the observation of nanoscale dynamics at physiologically relevant expression levels. Moreover, endogenous tagging of mitochondrial prohibitins allowed to gain insights into their previously unknown submitochondrial localization, protein dynamics and protein quantity on a single cell level.

While this work showed the integration of a single FP at one genomic locus, the established methodologies can be easily applied to incorporate a second FP at a second locus. Two color genomic tagging would allow protein co-localization studies at endogenous levels using (super-resolution) microscopy. This can be achieved by simultaneous or sequential cotransfection of two nuclease/donor plasmid pairs followed by sorting of single cells expressing both FPs.

Next to FPs, the integration of self-labelling enzymes (e.g. SNAP) would enable STED superresolution imaging on endogenous protein levels, because highly photostable organic dyes could be used. Moreover, self-labelling enzymes would allow novel experimental applications such as pulse-chase experiments to study protein turnover on physiological protein levels.

While protein tags such as FPs or SNAP tag are bulky structures that are usually placed at the $\mathrm{N}$ - or C-terminal end of a target protein, genetically encoded fluorescent amino acids are small and can be inserted also internally at many different positions of a polypeptide sequence (Lang and Chin, 2014). In combination with endogenous tagging, this labeling strategy should be the least disturbing one for most proteins. However, the incorporation of fluorescent amino acids into a protein of interest is still very inefficient and a few expert labs mainly use this labeling strategy so far.

The entire current protocol, from project planning until a characterized cell line is obtained, can be executed within about 6 weeks when U2OS or HeLa cells are used. Because recovery of these cells after single cell sorting takes about 2-3 weeks, using a cell line that displays a faster growth rate might accelerate the entire workflow.

Moreover, a strategy for selecting homozygous knock-in clones is lacking so far. This could be accomplished via integration of an antibiotic resistance gene downstream of the respective 
tag. The more copies of the respective gene are targeted, the more gene copies of the respective antibiotic resistance gene should be expressed from the genome. Therefore, applying increased antibiotic concentrations before single cell sorting might enrich homozygous knock-in clones.

The strategy for selecting homozygous knock-in clones should be applied to test whether prohibitin can be tagged homozygously with fluorescent proteins. This would enable to count copy numbers of prohibitin subunits in individual complexes or clusters using quantitative (super-resolution) imaging (Ulbrich, 2015).

The here presented labelling strategy based on CRISPR-mediated endogenous tagging of human proteins is superior to previous overexpression approaches. In combination with super-resolution imaging methods, endogenous tagging opens up a new field of quantitative nanoscopy that will allow unprecedented insights into a vast spectrum of biological applications. 


\section{References}

Alberts, B., Johnson, A., Lewis, J., Morgan, D., Raff, M., Roberts, K., and Walter, P. (2014). Molecular Biology of the Cell, Sixth Edition (Taylor \& Francis Group).

Ando, R., Mizuno, H., and Miyawaki, A. (2004). Regulated fast nucleocytoplasmic shuttling observed by reversible protein highlighting. Science 306, 1370-1373.

Andresen, M., Stiel, A.C., Folling, J., Wenzel, D., Schonle, A., Egner, A., Eggeling, C., Hell, S.W., and Jakobs, S. (2008). Photoswitchable fluorescent proteins enable monochromatic multilabel imaging and dual color fluorescence nanoscopy. Nat Biotechnol 26, 1035-1040.

Andresen, M., Stiel, A.C., Trowitzsch, S., Weber, G., Eggeling, C., Wahl, M.C., Hell, S.W., and Jakobs, S. (2007). Structural basis for reversible photoswitching in Dronpa. Proc Natl Acad Sci U S A 104, 13005-13009.

Andresen, M., Wahl, M.C., Stiel, A.C., Grater, F., Schafer, L.V., Trowitzsch, S., Weber, G., Eggeling, C., Grubmuller, H., Hell, S.W., et al. (2005). Structure and mechanism of the reversible photoswitch of a fluorescent protein. Proc Natl Acad Sci U S A 102, 13070-13074.

Artal-Sanz, M., and Tavernarakis, N. (2009). Prohibitin and mitochondrial biology. Trends Endocrinol Metab 20, 394-401.

Artal-Sanz, M., Tsang, W.Y., Willems, E.M., Grivell, L.A., Lemire, B.D., van der Spek, H., and Nijtmans, L.G. (2003). The mitochondrial prohibitin complex is essential for embryonic viability and germline function in Caenorhabditis elegans. J Biol Chem 278, 32091-32099.

Back, J.W., Sanz, M.A., De Jong, L., De Koning, L.J., Nijtmans, L.G., De Koster, C.G., Grivell, L.A., Van Der Spek, H., and Muijsers, A.O. (2002). A structure for the yeast prohibitin complex: Structure prediction and evidence from chemical crosslinking and mass spectrometry. Protein Sci 11, 2471-2478.

Barrangou, R., Fremaux, C., Deveau, H., Richards, M., Boyaval, P., Moineau, S., Romero, D.A., and Horvath, P. (2007). CRISPR provides acquired resistance against viruses in prokaryotes. Science 315, 1709-1712.

Barrangou, R., and Marraffini, L.A. (2014). CRISPR-Cas systems: Prokaryotes upgrade to adaptive immunity. Mol Cell 54, 234-244.

Barrett, L.W., Fletcher, S., and Wilton, S.D. (2012). Regulation of eukaryotic gene expression by the untranslated gene regions and other non-coding elements. Cell Mol Life Sci 69, 36133634.

Beckerle, M.C. (1997). Zyxin: zinc fingers at sites of cell adhesion. Bioessays 19, 949-957.

Ben-Shoshan, S.O., Simon, A.J., Jacob-Hirsch, J., Shaklai, S., Paz-Yaacov, N., Amariglio, N., Rechavi, G., and Trakhtenbrot, L. (2014). Induction of polyploidy by nuclear fusion mechanism upon decreased expression of the nuclear envelope protein LAP2beta in the human osteosarcoma cell line U2OS. Mol Cytogenet 7, 9. 
Berger, K.H., and Yaffe, M.P. (1998). Prohibitin family members interact genetically with mitochondrial inheritance components in Saccharomyces cerevisiae. Mol Cell Biol 18, 40434052.

Betzig, E., Patterson, G.H., Sougrat, R., Lindwasser, O.W., Olenych, S., Bonifacino, J.S., Davidson, M.W., Lippincott-Schwartz, J., and Hess, H.F. (2006). Imaging intracellular fluorescent proteins at nanometer resolution. Science 313, 1642-1645.

Bibikova, M., Beumer, K., Trautman, J.K., and Carroll, D. (2003). Enhancing gene targeting with designed zinc finger nucleases. Science 300, 764.

Bibikova, M., Carroll, D., Segal, D.J., Trautman, J.K., Smith, J., Kim, Y.G., and Chandrasegaran, S. (2001). Stimulation of homologous recombination through targeted cleavage by chimeric nucleases. Mol Cell Biol 21, 289-297.

Bibikova, M., Golic, M., Golic, K.G., and Carroll, D. (2002). Targeted chromosomal cleavage and mutagenesis in Drosophila using zinc-finger nucleases. Genetics 161, 1169-1175.

Birchler, J.A., and Veitia, R.A. (2012). Gene balance hypothesis: connecting issues of dosage sensitivity across biological disciplines. Proc Natl Acad Sci U S A 109, 14746-14753.

Birnboim, H.C., and Doly, J. (1979). A rapid alkaline extraction procedure for screening recombinant plasmid DNA. Nucleic Acids Res 7, 1513-1523.

Birner, R., Nebauer, R., Schneiter, R., and Daum, G. (2003). Synthetic lethal interaction of the mitochondrial phosphatidylethanolamine biosynthetic machinery with the prohibitin complex of Saccharomyces cerevisiae. Mol Biol Cell 14, 370-383.

Boch, J., Scholze, H., Schornack, S., Landgraf, A., Hahn, S., Kay, S., Lahaye, T., Nickstadt, A., and Bonas, U. (2009). Breaking the code of DNA binding specificity of TAL-type III effectors. Science 326, 1509-1512.

Bogdanove, A.J., and Voytas, D.F. (2011). TAL effectors: customizable proteins for DNA targeting. Science 333, 1843-1846.

Bolotin, A., Quinquis, B., Sorokin, A., and Ehrlich, S.D. (2005). Clustered regularly interspaced short palindrome repeats (CRISPRs) have spacers of extrachromosomal origin. Microbiology 151, 2551-2561.

Brakemann, T., Stiel, A.C., Weber, G., Andresen, M., Testa, I., Grotjohann, T., Leutenegger, M., Plessmann, U., Urlaub, H., Eggeling, C., et al. (2011). A reversibly photoswitchable GFPlike protein with fluorescence excitation decoupled from switching. Nat Biotechnol 29, 942947.

Brielmeier, M., Bechet, J.M., Falk, M.H., Pawlita, M., Polack, A., and Bornkamm, G.W. (1998). Improving stable transfection efficiency: antioxidants dramatically improve the outgrowth of clones under dominant marker selection. Nucleic Acids Res 26, 2082-2085.

Brouns, S.J., Jore, M.M., Lundgren, M., Westra, E.R., Slijkhuis, R.J., Snijders, A.P., Dickman, M.J., Makarova, K.S., Koonin, E.V., and van der Oost, J. (2008). Small CRISPR RNAs guide antiviral defense in prokaryotes. Science 321, 960-964. 
Browman, D.T., Hoegg, M.B., and Robbins, S.M. (2007). The SPFH domain-containing proteins: more than lipid raft markers. Trends Cell Biol 17, 394-402.

Burckstummer, T., Banning, C., Hainzl, P., Schobesberger, R., Kerzendorfer, C., Pauler, F.M., Chen, D., Them, N., Schischlik, F., Rebsamen, M., et al. (2013). A reversible gene trap collection empowers haploid genetics in human cells. Nat Methods 10, 965-971.

Capecchi, M.R. (1989). Altering the genome by homologous recombination. Science 244, 1288-1292.

Carette, J.E., Raaben, M., Wong, A.C., Herbert, A.S., Obernosterer, G., Mulherkar, N., Kuehne, A.l., Kranzusch, P.J., Griffin, A.M., Ruthel, G., et al. (2011). Ebola virus entry requires the cholesterol transporter Niemann-Pick C1. Nature 477, 340-343.

Carroll, D. (2011). Genome engineering with zinc-finger nucleases. Genetics 188, 773-782. Chalfie, M., Tu, Y., Euskirchen, G., Ward, W.W., and Prasher, D.C. (1994). Green fluorescent protein as a marker for gene expression. Science 263, 802-805.

Chen, B., Gilbert, L.A., Cimini, B.A., Schnitzbauer, J., Zhang, W., Li, G.W., Park, J., Blackburn, E.H., Weissman, J.S., Qi, L.S., et al. (2013). Dynamic imaging of genomic loci in living human cells by an optimized CRISPR/Cas system. Cell 155, 1479-1491.

Chmyrov, A., Keller, J., Grotjohann, T., Ratz, M., d'Este, E., Jakobs, S., Eggeling, C., and Hell, S.W. (2013). Nanoscopy with more than 100,000 'doughnuts'. Nat Methods 10, 737-740.

Cho, S.W., Kim, S., Kim, J.M., and Kim, J.S. (2013). Targeted genome engineering in human cells with the Cas9 RNA-guided endonuclease. Nat Biotechnol 31, 230-232.

Choudhary, C., Kumar, C., Gnad, F., Nielsen, M.L., Rehman, M., Walther, T.C., Olsen, J.V., and Mann, M. (2009). Lysine acetylation targets protein complexes and co-regulates major cellular functions. Science $325,834-840$.

Choulika, A., Perrin, A., Dujon, B., and Nicolas, J.F. (1995). Induction of homologous recombination in mammalian chromosomes by using the I-Scel system of Saccharomyces cerevisiae. Mol Cell Biol 15, 1968-1973.

Christian, M., Cermak, T., Doyle, E.L., Schmidt, C., Zhang, F., Hummel, A., Bogdanove, A.J., and Voytas, D.F. (2010). Targeting DNA double-strand breaks with TAL effector nucleases. Genetics 186, 757-761.

Christie, D.A., Lemke, C.D., Elias, I.M., Chau, L.A., Kirchhof, M.G., Li, B., Ball, E.H., Dunn, S.D., Hatch, G.M., and Madrenas, J. (2011). Stomatin-like protein 2 binds cardiolipin and regulates mitochondrial biogenesis and function. Mol Cell Biol 31, 3845-3856.

Christy, B., and Nathans, D. (1989). DNA binding site of the growth factor-inducible protein Zif268. Proc Natl Acad Sci U S A 86, 8737-8741.

Chu, V.T., Weber, T., Wefers, B., Wurst, W., Sander, S., Rajewsky, K., and Kuhn, R. (2015). Increasing the efficiency of homology-directed repair for CRISPR-Cas9-induced precise gene editing in mammalian cells. Nat Biotechnol 33, 543-548. 
Chudakov, D.M., Belousov, V.V., Zaraisky, A.G., Novoselov, V.V., Staroverov, D.B., Zorov, D.B., Lukyanov, S., and Lukyanov, K.A. (2003). Kindling fluorescent proteins for precise in vivo photolabeling. Nat Biotech 21, 191-194.

Chudakov, D.M., Matz, M.V., Lukyanov, S., and Lukyanov, K.A. (2010). Fluorescent proteins and their applications in imaging living cells and tissues. Physiol Rev 90, 1103-1163.

Cipolat, S., Martins de Brito, O., Dal Zilio, B., and Scorrano, L. (2004). OPA1 requires mitofusin 1 to promote mitochondrial fusion. Proc Natl Acad Sci U S A 101, 15927-15932.

Cipolat, S., Rudka, T., Hartmann, D., Costa, V., Serneels, L., Craessaerts, K., Metzger, K., Frezza, C., Annaert, W., D'Adamio, L., et al. (2006). Mitochondrial rhomboid PARL regulates cytochrome c release during apoptosis via OPA1-dependent cristae remodeling. Cell 126, 163-175.

Clop, A., Marcq, F., Takeda, H., Pirottin, D., Tordoir, X., Bibe, B., Bouix, J., Caiment, F., Elsen, J.M., Eychenne, F., et al. (2006). A mutation creating a potential illegitimate microRNA target site in the myostatin gene affects muscularity in sheep. Nat Genet 38, 813-818.

Coffman, V.C., and Wu, J.Q. (2014). Every laboratory with a fluorescence microscope should consider counting molecules. Mol Biol Cell 25, 1545-1548.

Cohen, R.N., van der Aa, M.A., Macaraeg, N., Lee, A.P., and Szoka, F.C., Jr. (2009). Quantification of plasmid DNA copies in the nucleus after lipoplex and polyplex transfection. J Control Release 135, 166-174.

Collins, T.J., Berridge, M.J., Lipp, P., and Bootman, M.D. (2002). Mitochondria are morphologically and functionally heterogeneous within cells. EMBO J 21, 1616-1627.

Cong, L., Ran, F.A., Cox, D., Lin, S., Barretto, R., Habib, N., Hsu, P.D., Wu, X., Jiang, W., Marraffini, L.A., et al. (2013). Multiplex genome engineering using CRISPR/Cas systems. Science 339, 819-823.

Cradick, T.J., Fine, E.J., Antico, C.J., and Bao, G. (2013). CRISPR/Cas9 systems targeting beta-globin and CCR5 genes have substantial off-target activity. Nucleic Acids Res 41, 95849592.

Da Cruz, S., Parone, P.A., Gonzalo, P., Bienvenut, W.V., Tondera, D., Jourdain, A., Quadroni, M., and Martinou, J.C. (2008). SLP-2 interacts with prohibitins in the mitochondrial inner membrane and contributes to their stability. Biochim Biophys Acta 1783, 904-911.

Dahlem, T.J., Hoshijima, K., Jurynec, M.J., Gunther, D., Starker, C.G., Locke, A.S., Weis, A.M., Voytas, D.F., and Grunwald, D.J. (2012). Simple methods for generating and detecting locus-specific mutations induced with TALENs in the zebrafish genome. PLoS Genet 8 , e1002861.

Day, R.N., and Davidson, M.W. (2009). The fluorescent protein palette: tools for cellular imaging. Chem Soc Rev 38, 2887-2921.

de Brito, O.M., and Scorrano, L. (2008). Mitofusin 2 tethers endoplasmic reticulum to mitochondria. Nature 456, 605-610. 
De Rosa, S.C., Herzenberg, L.A., Herzenberg, L.A., and Roederer, M. (2001). 11-color, 13parameter flow cytometry: identification of human naive $\mathrm{T}$ cells by phenotype, function, and Tcell receptor diversity. Nat Med 7, 245-248.

Dean, K.M., and Palmer, A.E. (2014). Advances in fluorescence labeling strategies for dynamic cellular imaging. Nat Chem Biol 10, 512-523.

Deltcheva, E., Chylinski, K., Sharma, C.M., Gonzales, K., Chao, Y., Pirzada, Z.A., Eckert, M.R., Vogel, J., and Charpentier, E. (2011). CRISPR RNA maturation by trans-encoded small RNA and host factor RNase III. Nature 471, 602-607.

Di Talia, S., Skotheim, J.M., Bean, J.M., Siggia, E.D., and Cross, F.R. (2007). The effects of molecular noise and size control on variability in the budding yeast cell cycle. Nature 448, 947-951.

Doudna, J.A., and Charpentier, E. (2014). Genome editing. The new frontier of genome engineering with CRISPR-Cas9. Science 346, 1258096.

Douglass, A.D., and Vale, R.D. (2005). Single-molecule microscopy reveals plasma membrane microdomains created by protein-protein networks that exclude or trap signaling molecules in T cells. Cell 121, 937-950.

Dow, L.E., Fisher, J., O'Rourke, K.P., Muley, A., Kastenhuber, E.R., Livshits, G., Tschaharganeh, D.F., Socci, N.D., and Lowe, S.W. (2015b). Inducible in vivo genome editing with CRISPR-Cas9. Nat Biotech advance online publication.

Dower, W.J., Miller, J.F., and Ragsdale, C.W. (1988). High efficiency transformation of E. coli by high voltage electroporation. Nucleic Acids Res 16, 6127-6145.

Doyon, J.B., Zeitler, B., Cheng, J., Cheng, A.T., Cherone, J.M., Santiago, Y., Lee, A.H., Vo, T.D., Doyon, Y., Miller, J.C., et al. (2011). Rapid and efficient clathrin-mediated endocytosis revealed in genome-edited mammalian cells. Nat Cell Biol 13, 331-337.

Dudek, J., Rehling, P., and van der Laan, M. (2013). Mitochondrial protein import: common principles and physiological networks. Biochim Biophys Acta 1833, 274-285.

Dyba, M., Jakobs, S., and Hell, S.W. (2003). Immunofluorescence stimulated emission depletion microscopy. Nat Biotechnol 21, 1303-1304.

Eggeling, C. (2015). Super-resolution optical microscopy of lipid plasma membrane dynamics. Essays Biochem 57, 69-80.

Elliott, B., Richardson, C., Winderbaum, J., Nickoloff, J.A., and Jasin, M. (1998). Gene conversion tracts from double-strand break repair in mammalian cells. Mol Cell Biol 18, 93101.

Elowitz, M.B., Levine, A.J., Siggia, E.D., and Swain, P.S. (2002). Stochastic gene expression in a single cell. Science 297, 1183-1186. 
Fetter, J., Samsonov, A., Zenser, N., Zhang, F., Zhang, H., and Malkov, D. (2015). Endogenous gene tagging with fluorescent proteins. Methods in Molecular Biology 1239, 231240.

Frezza, C., Cipolat, S., Martins de Brito, O., Micaroni, M., Beznoussenko, G.V., Rudka, T., Bartoli, D., Polishuck, R.S., Danial, N.N., De Strooper, B., et al. (2006). OPA1 controls apoptotic cristae remodeling independently from mitochondrial fusion. Cell 126, 177-189.

Friedland, A.E., Tzur, Y.B., Esvelt, K.M., Colaiacovo, M.P., Church, G.M., and Calarco, J.A. (2013). Heritable genome editing in C. elegans via a CRISPR-Cas9 system. Nat Methods 10, 741-743.

Frock, R.L., Hu, J., Meyers, R.M., Ho, Y.J., Kii, E., and Alt, F.W. (2015). Genome-wide detection of DNA double-stranded breaks induced by engineered nucleases. Nat Biotechnol 33, 179-186.

Fu, Y., Foden, J.A., Khayter, C., Maeder, M.L., Reyon, D., Joung, J.K., and Sander, J.D. (2013). High-frequency off-target mutagenesis induced by CRISPR-Cas nucleases in human cells. Nat Biotechnol 31, 822-826.

Fu, Y., Sander, J.D., Reyon, D., Cascio, V.M., and Joung, J.K. (2014). Improving CRISPRCas nuclease specificity using truncated guide RNAs. Nat Biotechnol.

Fuchs, E., and Cleveland, D.W. (1998). A structural scaffolding of intermediate filaments in health and disease. Science 279, 514-519.

Fuchs, E., and Weber, K. (1994). Intermediate filaments: structure, dynamics, function, and disease. Annu Rev Biochem 63, 345-382.

Fusaro, G., Dasgupta, P., Rastogi, S., Joshi, B., and Chellappan, S. (2003). Prohibitin induces the transcriptional activity of p53 and is exported from the nucleus upon apoptotic signaling. J Biol Chem 278, 47853-47861.

Gantz, V.M., and Bier, E. (2015). Genome editing. The mutagenic chain reaction: a method for converting heterozygous to homozygous mutations. Science 348, 442-444.

Gasiunas, G., Barrangou, R., Horvath, P., and Siksnys, V. (2012). Cas9-crRNA ribonucleoprotein complex mediates specific DNA cleavage for adaptive immunity in bacteria. Proc Natl Acad Sci U S A 109, E2579-2586.

Ghaemmaghami, S., Huh, W.K., Bower, K., Howson, R.W., Belle, A., Dephoure, N., O'Shea, E.K., and Weissman, J.S. (2003). Global analysis of protein expression in yeast. Nature 425, 737-741.

Gibson, D.G., Young, L., Chuang, R.Y., Venter, J.C., Hutchison, C.A., 3rd, and Smith, H.O. (2009). Enzymatic assembly of DNA molecules up to several hundred kilobases. Nat Methods 6, 343-345.

Gibson, T.J., Seiler, M., and Veitia, R.A. (2013). The transience of transient overexpression. Nat Methods 10, 715-721. 
Gilbert, L.A., Larson, M.H., Morsut, L., Liu, Z., Brar, G.A., Torres, S.E., Stern-Ginossar, N., Brandman, O., Whitehead, E.H., Doudna, J.A., et al. (2013). CRISPR-mediated modular RNA-guided regulation of transcription in eukaryotes. Cell 154, 442-451.

Godin, Antoine G., Lounis, B., and Cognet, L. (2014). Super-resolution Microscopy Approaches for Live Cell Imaging. Biophysical Journal 107, 1777-1784.

Gomes, L.C., Di Benedetto, G., and Scorrano, L. (2011). During autophagy mitochondria elongate, are spared from degradation and sustain cell viability. Nat Cell Biol 13, 589-598.

Gonzalez, B., Schwimmer, L.J., Fuller, R.P., Ye, Y., Asawapornmongkol, L., and Barbas, C.F., 3rd (2010). Modular system for the construction of zinc-finger libraries and proteins. Nat Protoc 5, 791-810.

Griparic, L., van der Wel, N.N., Orozco, I.J., Peters, P.J., and van der Bliek, A.M. (2004). Loss of the intermembrane space protein Mgm1/OPA1 induces swelling and localized constrictions along the lengths of mitochondria. J Biol Chem 279, 18792-18798.

Grosschedl, R., Giese, K., and Pagel, J. (1994). HMG domain proteins: architectural elements in the assembly of nucleoprotein structures. Trends Genet 10, 94-100.

Grotjohann, T., Testa, I., Leutenegger, M., Bock, H., Urban, N.T., Lavoie-Cardinal, F., Willig, K.I., Eggeling, C., Jakobs, S., and Hell, S.W. (2011). Diffraction-unlimited all-optical imaging and writing with a photochromic GFP. Nature 478, 204-208.

Grotjohann, T., Testa, I., Reuss, M., Brakemann, T., Eggeling, C., Hell, S.W., and Jakobs, S. (2012). rsEGFP2 enables fast RESOLFT nanoscopy of living cells. Elife 1, e00248.

Guilinger, J.P., Thompson, D.B., and Liu, D.R. (2014). Fusion of catalytically inactive Cas9 to Fokl nuclease improves the specificity of genome modification. Nat Biotechnol 32, 577-582.

Guschin, D.Y., Waite, A.J., Katibah, G.E., Miller, J.C., Holmes, M.C., and Rebar, E.J. (2010). $A$ rapid and general assay for monitoring endogenous gene modification. Methods Mol Biol $649,247-256$.

Haft, D.H., Selengut, J., Mongodin, E.F., and Nelson, K.E. (2005). A guild of 45 CRISPRassociated (Cas) protein families and multiple CRISPR/Cas subtypes exist in prokaryotic genomes. PLoS Comput Biol 1, e60.

Hajek, P., Chomyn, A., and Attardi, G. (2007). Identification of a novel mitochondrial complex containing mitofusin 2 and stomatin-like protein 2. J Biol Chem 282, 5670-5681.

Hale, C.R., Zhao, P., Olson, S., Duff, M.O., Graveley, B.R., Wells, L., Terns, R.M., and Terns, M.P. (2009). RNA-guided RNA cleavage by a CRISPR RNA-Cas protein complex. Cell 139, 945-956.

Halff, E.F., Versteeg, M., Brondijk, T.H., and Huizinga, E.G. (2014). When less becomes more: optimization of protein expression in HEK293-EBNA1 cells using plasmid titration - a case study for NLRs. Protein Expr Purif 99, 27-34. 
Halling-Brown, M.D., Bulusu, K.C., Patel, M., Tym, J.E., and Al-Lazikani, B. (2012). canSAR: an integrated cancer public translational research and drug discovery resource. Nucleic Acids Res 40, D947-956.

Hanahan, D., Jessee, J., and Bloom, F.R. (1991). Plasmid transformation of Escherichia coli and other bacteria. Methods Enzymol 204, 63-113.

Hasty, P., Rivera-Perez, J., and Bradley, A. (1991). The length of homology required for gene targeting in embryonic stem cells. Mol Cell Biol 11, 5586-5591.

Hayflick, L. (1965). The Limited in Vitro Lifetime of Human Diploid Cell Strains. Exp Cell Res 37, 614-636.

Hayflick, L., and Moorhead, P.S. (1961). The serial cultivation of human diploid cell strains. Exp Cell Res 25, 585-621.

Heim, R., Prasher, D.C., and Tsien, R.Y. (1994). Wavelength mutations and posttranslational autoxidation of green fluorescent protein. Proc Natl Acad Sci U S A 91, 12501-12504.

Hell, S.W., and Wichmann, J. (1994). Breaking the diffraction resolution limit by stimulated emission: stimulated-emission-depletion fluorescence microscopy. Opt Lett 19, 780-782.

Hell, S.W., and Kroug, M. (1995). Ground-state-depletion fluorescence microscopy: a concept for breaking the diffraction resolution limit. Applied Physics B: Lasers and Optics 60, 495-497.

Hell, S.W. (2003). Toward fluorescence nanoscopy. Nat Biotechnol 21, 1347-1355.

Hell, S.W. (2007). Far-field optical nanoscopy. Science 316, 1153-1158.

Hell, S.W. (2009). Far-Field Optical Nanoscopy. In Single Molecule Spectroscopy in Chemistry, Physics and Biology, A. Gräslund, R. Rigler, and J. Widengren, eds. (Berlin: Springer), pp. 298-365.

Herrmann, J.M., and Riemer, J. (2010). The intermembrane space of mitochondria. Antioxid Redox Signal 13, 1341-1358.

Hess, S.T., Girirajan, T.P., and Mason, M.D. (2006). Ultra-high resolution imaging by fluorescence photoactivation localization microscopy. Biophys J 91, 4258-4272.

Hilton, I.B., D'Ippolito, A.M., Vockley, C.M., Thakore, P.I., Crawford, G.E., Reddy, T.E., and Gersbach, C.A. (2015). Epigenome editing by a CRISPR-Cas9-based acetyltransferase activates genes from promoters and enhancers. Nat Biotechnol 33, 510-517.

Hindson, B.J., Ness, K.D., Masquelier, D.A., Belgrader, P., Heredia, N.J., Makarewicz, A.J., Bright, I.J., Lucero, M.Y., Hiddessen, A.L., Legler, T.C., et al. (2011). High-throughput droplet digital PCR system for absolute quantitation of DNA copy number. Anal Chem 83, 8604-8610.

Hockemeyer, D., Wang, H., Kiani, S., Lai, C.S., Gao, Q., Cassady, J.P., Cost, G.J., Zhang, L., Santiago, Y., Miller, J.C., et al. (2011). Genetic engineering of human pluripotent cells using TALE nucleases. Nat Biotechnol 29, 731-734. 
Hofmann, M., Eggeling, C., Jakobs, S., and Hell, S.W. (2005). Breaking the diffraction barrier in fluorescence microscopy at low light intensities by using reversibly photoswitchable proteins. Proc Natl Acad Sci U S A 102, 17565-17569.

Hoppins, S., Lackner, L., and Nunnari, J. (2007). The machines that divide and fuse mitochondria. Annu Rev Biochem 76, 751-780.

Hsu, P.D., Lander, E.S., and Zhang, F. (2014). Development and applications of CRISPRCas9 for genome engineering. Cell 157, 1262-1278.

Hsu, P.D., Scott, D.A., Weinstein, J.A., Ran, F.A., Konermann, S., Agarwala, V., Li, Y., Fine, E.J., Wu, X., Shalem, O., et al. (2013). DNA targeting specificity of RNA-guided Cas9 nucleases. Nat Biotechnol 31, 827-832.

Huber, T.B., Schermer, B., Muller, R.U., Hohne, M., Bartram, M., Calixto, A., Hagmann, H., Reinhardt, C., Koos, F., Kunzelmann, K., et al. (2006). Podocin and MEC-2 bind cholesterol to regulate the activity of associated ion channels. Proc Natl Acad Sci U S A 103, 1707917086.

Huh, W.K., Falvo, J.V., Gerke, L.C., Carroll, A.S., Howson, R.W., Weissman, J.S., and O'Shea, E.K. (2003). Global analysis of protein localization in budding yeast. Nature 425, 686-691.

Hung, M.C., and Link, W. (2011). Protein localization in disease and therapy. J Cell Sci 124, 3381-3392.

Hwang, W.Y., Fu, Y., Reyon, D., Maeder, M.L., Tsai, S.Q., Sander, J.D., Peterson, R.T., Yeh, J.R., and Joung, J.K. (2013). Efficient genome editing in zebrafish using a CRISPR-Cas system. Nat Biotechnol 31, 227-229.

Ikonen, E., Fiedler, K., Parton, R.G., and Simons, K. (1995). Prohibitin, an antiproliferative protein, is localized to mitochondria. FEBS Lett 358, 273-277.

Inouye, S., and Tsuji, F.I. (1994). Aequorea green fluorescent protein. Expression of the gene and fluorescence characteristics of the recombinant protein. FEBS Lett 341, 277-280.

Ishihara, N., Fujita, Y., Oka, T., and Mihara, K. (2006). Regulation of mitochondrial morphology through proteolytic cleavage of OPA1. EMBO J 25, 2966-2977.

Ishino, Y., Shinagawa, H., Makino, K., Amemura, M., and Nakata, A. (1987). Nucleotide sequence of the iap gene, responsible for alkaline phosphatase isozyme conversion in Escherichia coli, and identification of the gene product. J Bacteriol 169, 5429-5433.

Jamal, M., Khan, F.A., Da, L., Habib, Z., Dai, J., and Cao, G. (2015). Keeping CRISPR/Cas on-Target. Curr Issues Mol Biol 20, 1-20.

Jansen, R., Embden, J.D., Gaastra, W., and Schouls, L.M. (2002). Identification of genes that are associated with DNA repeats in prokaryotes. Mol Microbiol 43, 1565-1575.

Janssen, A., and Medema, R.H. (2013). Genetic instability: tipping the balance. Oncogene 32, 4459-4470. 
Jasin, M., and Rothstein, R. (2013). Repair of strand breaks by homologous recombination. Cold Spring Harb Perspect Biol 5, a012740.

Jensen, N.A., Danzl, J.G., Willig, K.I., Lavoie-Cardinal, F., Brakemann, T., Hell, S.W., and Jakobs, S. (2014). Coordinate-targeted and coordinate-stochastic super-resolution microscopy with the reversibly switchable fluorescent protein dreiklang. Chemphyschem 15, 756-762.

Jiang, W., Bikard, D., Cox, D., Zhang, F., and Marraffini, L.A. (2013). RNA-guided editing of bacterial genomes using CRISPR-Cas systems. Nat Biotechnol 31, 233-239.

Jinek, M., Chylinski, K., Fonfara, I., Hauer, M., Doudna, J.A., and Charpentier, E. (2012). A programmable dual-RNA-guided DNA endonuclease in adaptive bacterial immunity. Science $337,816-821$.

Jinek, M., East, A., Cheng, A., Lin, S., Ma, E., and Doudna, J. (2013). RNA-programmed genome editing in human cells. Elife 2, e00471.

Joung, J.K., and Sander, J.D. (2013). TALENs: a widely applicable technology for targeted genome editing. Nat Rev Mol Cell Biol 14, 49-55.

Juillerat, A., Dubois, G., Valton, J., Thomas, S., Stella, S., Marechal, A., Langevin, S., Benomari, N., Bertonati, C., Silva, G.H., et al. (2014). Comprehensive analysis of the specificity of transcription activator-like effector nucleases. Nucleic Acids Res 42, 5390-5402.

Jupe, E.R., Liu, X.T., Kiehlbauch, J.L., McClung, J.K., and Dell'Orco, R.T. (1996). The 3' untranslated region of prohibitin and cellular immortalization. Exp Cell Res 224, 128-135.

Kaern, M., Elston, T.C., Blake, W.J., and Collins, J.J. (2005). Stochasticity in gene expression: from theories to phenotypes. Nat Rev Genet 6, 451-464.

Kao, Y.T., Zhu, X., and Min, W. (2012). Protein-flexibility mediated coupling between photoswitching kinetics and surrounding viscosity of a photochromic fluorescent protein. Proc Natl Acad Sci U S A 109, 3220-3225.

Kasahara, A., and Scorrano, L. (2014). Mitochondria: from cell death executioners to regulators of cell differentiation. Trends in Cell Biology 24, 761-770.

Kasashima, K., Ohta, E., Kagawa, Y., and Endo, H. (2006). Mitochondrial functions and estrogen receptor-dependent nuclear translocation of pleiotropic human prohibitin 2. J Biol Chem 281, 36401-36410.

Kiani, S., Beal, J., Ebrahimkhani, M.R., Huh, J., Hall, R.N., Xie, Z., Li, Y., and Weiss, R. (2014). CRISPR transcriptional repression devices and layered circuits in mammalian cells. Nat Methods 11, 723-726.

Kim, D., Bae, S., Park, J., Kim, E., Kim, S., Yu, H.R., Hwang, J., Kim, J.I., and Kim, J.S. (2015). Digenome-seq: genome-wide profiling of CRISPR-Cas9 off-target effects in human cells. Nat Methods 12, 237-243, 231 p following 243. 
Kim, S., Kim, D., Cho, S.W., Kim, J., and Kim, J.S. (2014). Highly efficient RNA-guided genome editing in human cells via delivery of purified Cas9 ribonucleoproteins. Genome Res 24, 1012-1019.

Kim, Y.G., Cha, J., and Chandrasegaran, S. (1996). Hybrid restriction enzymes: zinc finger fusions to Fok I cleavage domain. Proc Natl Acad Sci U S A 93, 1156-1160.

Klar, T.A., Jakobs, S., Dyba, M., Egner, A., and Hell, S.W. (2000). Fluorescence microscopy with diffraction resolution barrier broken by stimulated emission. Proc Natl Acad Sci U S A 97, 8206-8210.

Kober, L., Zehe, C., and Bode, J. (2012). Development of a novel ER stress based selection system for the isolation of highly productive clones. Biotechnol Bioeng 109, 2599-2611.

Koike-Yusa, H., Li, Y., Tan, E.P., Velasco-Herrera Mdel, C., and Yusa, K. (2014). Genomewide recessive genetic screening in mammalian cells with a lentiviral CRISPR-guide RNA library. Nat Biotechnol 32, 267-273.

Kolmakov, K., Belov, V.N., Bierwagen, J., Ringemann, C., Muller, V., Eggeling, C., and Hell, S.W. (2010). Red-emitting rhodamine dyes for fluorescence microscopy and nanoscopy. Chemistry 16, 158-166.

Kolonin, M.G., Saha, P.K., Chan, L., Pasqualini, R., and Arap, W. (2004). Reversal of obesity by targeted ablation of adipose tissue. Nat Med 10, 625-632.

Konermann, S., Brigham, M.D., Trevino, A.E., Hsu, P.D., Heidenreich, M., Cong, L., Platt, R.J., Scott, D.A., Church, G.M., and Zhang, F. (2013). Optical control of mammalian endogenous transcription and epigenetic states. Nature 500, 472-476.

Kornmann, B., Currie, E., Collins, S.R., Schuldiner, M., Nunnari, J., Weissman, J.S., and Walter, P. (2009). An ER-mitochondria tethering complex revealed by a synthetic biology screen. Science 325, 477-481.

Koshiba, T., Detmer, S.A., Kaiser, J.T., Chen, H., McCaffery, J.M., and Chan, D.C. (2004). Structural basis of mitochondrial tethering by mitofusin complexes. Science 305, 858-862.

Kowno, M., Watanabe-Susaki, K., Ishimine, H., Komazaki, S., Enomoto, K., Seki, Y., Wang, Y.Y., Ishigaki, Y., Ninomiya, N., Noguchi, T.A., et al. (2014). Prohibitin 2 regulates the proliferation and lineage-specific differentiation of mouse embryonic stem cells in mitochondria. PLoS One 9, e81552.

Kurtev, V., Margueron, R., Kroboth, K., Ogris, E., Cavailles, V., and Seiser, C. (2004). Transcriptional regulation by the repressor of estrogen receptor activity via recruitment of histone deacetylases. J Biol Chem 279, 24834-24843.

Kuscu, C., Arslan, S., Singh, R., Thorpe, J., and Adli, M. (2014). Genome-wide analysis reveals characteristics of off-target sites bound by the Cas9 endonuclease. Nat Biotechnol $32,677-683$. 
Kushner, P.J., Hort, E., Shine, J., Baxter, J.D., and Greene, G.L. (1990). Construction of cell lines that express high levels of the human estrogen receptor and are killed by estrogens. Mol Endocrinol 4, 1465-1473.

Kuznetsov, A.V., Schneeberger, S., Renz, O., Meusburger, H., Saks, V., Usson, Y., and Margreiter, R. (2004). Functional heterogeneity of mitochondria after cardiac cold ischemia and reperfusion revealed by confocal imaging. Transplantation 77, 754-756.

Lakowicz, J.R. (2007). Principles of Fluorescence Spectroscopy (Springer US).

Lang, K., and Chin, J.W. (2014). Cellular incorporation of unnatural amino acids and bioorthogonal labeling of proteins. Chem Rev 114, 4764-4806.

Lavoie-Cardinal, F., Jensen, N.A., Westphal, V., Stiel, A.C., Chmyrov, A., Bierwagen, J., Testa, I., Jakobs, S., and Hell, S.W. (2014). Two-Color RESOLFT Nanoscopy with Green and Red Fluorescent Photochromic Proteins. Chemphyschem 15, 655-663.

Lazarou, M., Sliter, D.A., Kane, L.A., Sarraf, S.A., Wang, C., Burman, J.L., Sideris, D.P., Fogel, A.I., and Youle, R.J. (2015). The ubiquitin kinase PINK1 recruits autophagy receptors to induce mitophagy. Nature 524, 309-314.

Liang, F., and Jasin, M. (1995). Studies on the influence of cytosine methylation on DNA recombination and end-joining in mammalian cells. J Biol Chem 270, 23838-23844.

Lin, S., Staahl, B.T., Alla, R.K., and Doudna, J.A. (2014). Enhanced homology-directed human genome engineering by controlled timing of CRISPR/Cas9 delivery. Elife 3, e04766. Lodish, H. (2008). Molecular Cell Biology (W. H. Freeman).

Lukinavicius, G., Reymond, L., D'Este, E., Masharina, A., Gottfert, F., Ta, H., Guther, A., Fournier, M., Rizzo, S., Waldmann, H., et al. (2014). Fluorogenic probes for live-cell imaging of the cytoskeleton. Nat Methods 11, 731-733.

Maeder, M.L., Linder, S.J., Cascio, V.M., Fu, Y., Ho, Q.H., and Joung, J.K. (2013). CRISPR RNA-guided activation of endogenous human genes. Nat Methods 10, 977-979.

Maeder, M.L., Thibodeau-Beganny, S., Osiak, A., Wright, D.A., Anthony, R.M., Eichtinger, M., Jiang, T., Foley, J.E., Winfrey, R.J., Townsend, J.A., et al. (2008). Rapid "open-source" engineering of customized zinc-finger nucleases for highly efficient gene modification. Mol Cell 31, 294-301.

Makarova, K.S., Haft, D.H., Barrangou, R., Brouns, S.J., Charpentier, E., Horvath, P., Moineau, S., Mojica, F.J., Wolf, Y.I., Yakunin, A.F., et al. (2011). Evolution and classification of the CRISPR-Cas systems. Nat Rev Microbiol 9, 467-477.

Mali, P., Yang, L., Esvelt, K.M., Aach, J., Guell, M., DiCarlo, J.E., Norville, J.E., and Church, G.M. (2013). RNA-guided human genome engineering via Cas9. Science 339, 823-826.

Mansour, S.L., Thomas, K.R., and Capecchi, M.R. (1988). Disruption of the proto-oncogene int-2 in mouse embryo-derived stem cells: a general strategy for targeting mutations to nonselectable genes. Nature 336, 348-352. 
Marraffini, L.A., and Sontheimer, E.J. (2008). CRISPR interference limits horizontal gene transfer in staphylococci by targeting DNA. Science 322, 1843-1845.

Mashal, R.D., Koontz, J., and Sklar, J. (1995). Detection of mutations by cleavage of DNA heteroduplexes with bacteriophage resolvases. Nat Genet 9, 177-183.

McClung, J.K., Danner, D.B., Stewart, D.A., Smith, J.R., Schneider, E.L., Lumpkin, C.K., Dell'Orco, R.T., and Nuell, M.J. (1989). Isolation of a cDNA that hybrid selects antiproliferative mRNA from rat liver. Biochem Biophys Res Commun 164, 1316-1322.

Mears, J.A., Lackner, L.L., Fang, S., Ingerman, E., Nunnari, J., and Hinshaw, J.E. (2011). Conformational changes in Dnm1 support a contractile mechanism for mitochondrial fission. Nat Struct Mol Biol 18, 20-26.

Mendez, M.G., Kojima, S., and Goldman, R.D. (2010). Vimentin induces changes in cell shape, motility, and adhesion during the epithelial to mesenchymal transition. FASEB J 24 , 1838-1851.

Merkwirth, C., Dargazanli, S., Tatsuta, T., Geimer, S., Lower, B., Wunderlich, F.T., von KleistRetzow, J.C., Waisman, A., Westermann, B., and Langer, T. (2008). Prohibitins control cell proliferation and apoptosis by regulating OPA1-dependent cristae morphogenesis in mitochondria. Genes Dev 22, 476-488.

Merkwirth, C., and Langer, T. (2009). Prohibitin function within mitochondria: essential roles for cell proliferation and cristae morphogenesis. Biochim Biophys Acta 1793, 27-32.

Miller, J.C., Tan, S., Qiao, G., Barlow, K.A., Wang, J., Xia, D.F., Meng, X., Paschon, D.E., Leung, E., Hinkley, S.J., et al. (2011). A TALE nuclease architecture for efficient genome editing. Nat Biotechnol 29, 143-148.

Mishra, S., Murphy, L.C., and Murphy, L.J. (2006). The Prohibitins: emerging roles in diverse functions. J Cell Mol Med 10, 353-363.

Mishra, S., Murphy, L.C., Nyomba, B.L., and Murphy, L.J. (2005). Prohibitin: a potential target for new therapeutics. Trends Mol Med 11, 192-197.

Mojica, F.J., Diez-Villasenor, C., Garcia-Martinez, J., and Soria, E. (2005). Intervening sequences of regularly spaced prokaryotic repeats derive from foreign genetic elements. $\mathrm{J}$ Mol Evol 60, 174-182.

Mojica, F.J., Diez-Villasenor, C., Soria, E., and Juez, G. (2000). Biological significance of a family of regularly spaced repeats in the genomes of Archaea, Bacteria and mitochondria. Mol Microbiol 36, 244-246.

Mollet, M., Godoy-Silva, R., Berdugo, C., and Chalmers, J.J. (2007). Acute hydrodynamic forces and apoptosis: a complex question. Biotechnol Bioeng 98, 772-788.

Montano, M.M., Ekena, K., Delage-Mourroux, R., Chang, W., Martini, P., and Katzenellenbogen, B.S. (1999). An estrogen receptor-selective coregulator that potentiates the effectiveness of antiestrogens and represses the activity of estrogens. Proc Natl Acad Sci U S A 96, 6947-6952. 
Moreno-Mateos, M.A., Vejnar, C.E., Beaudoin, J.D., Fernandez, J.P., Mis, E.K., Khokha, M.K., and Giraldez, A.J. (2015). CRISPRscan: designing highly efficient sgRNAs for CRISPRCas9 targeting in vivo. Nat Methods 12, 982-988.

Moscou, M.J., and Bogdanove, A.J. (2009). A simple cipher governs DNA recognition by TAL effectors. Science 326, 1501.

Mounier, N., Perriard, J.C., Gabbiani, G., and Chaponnier, C. (1997). Transfected muscle and non-muscle actins are differentially sorted by cultured smooth muscle and non-muscle cells. J Cell Sci 110 ( Pt 7), 839-846.

Niemisto, A., Dunmire, V., Yli-Harja, O., Zhang, W., and Shmulevich, I. (2005). Robust quantification of in vitro angiogenesis through image analysis. IEEE Trans Med Imaging 24, 549-553.

Nienhaus, K., and Nienhaus, G.U. (2014). Fluorescent proteins for live-cell imaging with super-resolution. Chem Soc Rev 43, 1088-1106.

Nihongaki, Y., Kawano, F., Nakajima, T., and Sato, M. (2015). Photoactivatable CRISPRCas9 for optogenetic genome editing. Nat Biotechnol 33, 755-760.

Nijtmans, L.G., de Jong, L., Artal Sanz, M., Coates, P.J., Berden, J.A., Back, J.W., Muijsers, A.O., van der Spek, H., and Grivell, L.A. (2000). Prohibitins act as a membrane-bound chaperone for the stabilization of mitochondrial proteins. EMBO J 19, 2444-2451.

Nishio, K., Inoue, A., Qiao, S., Kondo, H., and Mimura, A. (2001). Senescence and cytoskeleton: overproduction of vimentin induces senescent-like morphology in human fibroblasts. Histochem Cell Biol 116, 321-327.

Nissim, L., Perli, S.D., Fridkin, A., Perez-Pinera, P., and Lu, T.K. (2014). Multiplexed and programmable regulation of gene networks with an integrated RNA and CRISPR/Cas toolkit in human cells. Mol Cell 54, 698-710.

Nix, D.A., Fradelizi, J., Bockholt, S., Menichi, B., Louvard, D., Friederich, E., and Beckerle, M.C. (2001). Targeting of zyxin to sites of actin membrane interaction and to the nucleus. J Biol Chem 276, 34759-34767.

Nunnari, J., and Suomalainen, A. (2012). Mitochondria: In Sickness and in Health. Cell 148, 1145-1159.

Olsen, J.V., Blagoev, B., Gnad, F., Macek, B., Kumar, C., Mortensen, P., and Mann, M. (2006). Global, in vivo, and site-specific phosphorylation dynamics in signaling networks. Cell $127,635-648$.

Ormo, M., Cubitt, A.B., Kallio, K., Gross, L.A., Tsien, R.Y., and Remington, S.J. (1996). Crystal structure of the Aequorea victoria green fluorescent protein. Science 273, 1392-1395.

Osman, C., Haag, M., Potting, C., Rodenfels, J., Dip, P.V., Wieland, F.T., Brugger, B., Westermann, B., and Langer, T. (2009a). The genetic interactome of prohibitins: coordinated control of cardiolipin and phosphatidylethanolamine by conserved regulators in mitochondria. J Cell Biol 184, 583-596. 
Osman, C., Merkwirth, C., and Langer, T. (2009b). Prohibitins and the functional compartmentalization of mitochondrial membranes. Journal of Cell Science 122, 3823-3830.

Palade, G.E. (1952). The fine structure of mitochondria. Anat Rec 114, 427-451.

Partikian, A., Olveczky, B., Swaminathan, R., Li, Y., and Verkman, A.S. (1998). Rapid diffusion of green fluorescent protein in the mitochondrial matrix. J Cell Biol 140, 821-829.

Pattanayak, V., Lin, S., Guilinger, J.P., Ma, E., Doudna, J.A., and Liu, D.R. (2013). Highthroughput profiling of off-target DNA cleavage reveals RNA-programmed Cas9 nuclease specificity. Nat Biotechnol 31, 839-843.

Pavletich, N.P., and Pabo, C.O. (1991). Zinc finger-DNA recognition: crystal structure of a Zif268-DNA complex at 2.1 A. Science 252, 809-817.

Pawley, J. (2013). Handbook of Biological Confocal Microscopy (Springer US).

Peckham, M., Miller, G., Wells, C., Zicha, D., and Dunn, G.A. (2001). Specific changes to the mechanism of cell locomotion induced by overexpression of beta-actin. J Cell Sci 114, 13671377.

Perez-Pinera, P., Kocak, D.D., Vockley, C.M., Adler, A.F., Kabadi, A.M., Polstein, L.R., Thakore, P.I., Glass, K.A., Ousterout, D.G., Leong, K.W., et al. (2013). RNA-guided gene activation by CRISPR-Cas9-based transcription factors. Nat Methods 10, 973-976.

Pike, L.J. (2009). The challenge of lipid rafts. J Lipid Res 50 Suppl, S323-328.

Plessis, A., Perrin, A., Haber, J.E., and Dujon, B. (1992). Site-specific recombination determined by I-Scel, a mitochondrial group I intron-encoded endonuclease expressed in the yeast nucleus. Genetics 130, 451-460.

Polstein, L.R., and Gersbach, C.A. (2015). A light-inducible CRISPR-Cas9 system for control of endogenous gene activation. Nat Chem Biol 11, 198-200.

Ponten, J., and Saksela, E. (1967). Two established in vitro cell lines from human mesenchymal tumours. Int J Cancer 2, 434-447.

Port, F., Muschalik, N., and Bullock, S.L. (2015). Systematic Evaluation of Drosophila CRISPR Tools Reveals Safe and Robust Alternatives to Autonomous Gene Drives in Basic Research. G3 (Bethesda) 5, 1493-1502.

Prasher, D.C., Eckenrode, V.K., Ward, W.W., Prendergast, F.G., and Cormier, M.J. (1992). Primary structure of the Aequorea victoria green-fluorescent protein. Gene 111, 229-233.

Puchner, E.M., Walter, J.M., Kasper, R., Huang, B., and Lim, W.A. (2013). Counting molecules in single organelles with superresolution microscopy allows tracking of the endosome maturation trajectory. Proc Natl Acad Sci U S A 110, 16015-16020.

Qi, L.S., Larson, M.H., Gilbert, L.A., Doudna, J.A., Weissman, J.S., Arkin, A.P., and Lim, W.A. (2013). Repurposing CRISPR as an RNA-guided platform for sequence-specific control of gene expression. Cell 152, 1173-1183. 
Ramakrishna, S., Kwaku Dad, A.B., Beloor, J., Gopalappa, R., Lee, S.K., and Kim, H. (2014). Gene disruption by cell-penetrating peptide-mediated delivery of Cas9 protein and guide RNA. Genome Res 24, 1020-1027.

Ramdas, J., and Muniyappa, K. (1995). Recognition and alignment of homologous DNA sequences between minichromosomes and single-stranded DNA promoted by RecA protein. Mol Gen Genet 249, 336-348.

Ran, F.A., Hsu, P.D., Lin, C.Y., Gootenberg, J.S., Konermann, S., Trevino, A.E., Scott, D.A., Inoue, A., Matoba, S., Zhang, Y., et al. (2013a). Double nicking by RNA-guided CRISPR Cas9 for enhanced genome editing specificity. Cell 154, 1380-1389.

Ran, F.A., Hsu, P.D., Wright, J., Agarwala, V., Scott, D.A., and Zhang, F. (2013b). Genome engineering using the CRISPR-Cas9 system. Nat Protoc 8, 2281-2308.

Ratz, M., Testa, I., Hell, S.W., and Jakobs, S. (2015). CRISPR/Cas9-mediated endogenous protein tagging for RESOLFT super-resolution microscopy of living human cells. Sci Rep 5, 9592.

Reinius, B., and Sandberg, R. (2015). Random monoallelic expression of autosomal genes: stochastic transcription and allele-level regulation. Nat Rev Genet 16, 653-664.

Riedl, J., Crevenna, A.H., Kessenbrock, K., Yu, J.H., Neukirchen, D., Bista, M., Bradke, F., Jenne, D., Holak, T.A., Werb, Z., et al. (2008). Lifeact: a versatile marker to visualize F-actin. Nat Methods 5, 605-607.

Ries, J., Kaplan, C., Platonova, E., Eghlidi, H., and Ewers, H. (2012). A simple, versatile method for GFP-based super-resolution microscopy via nanobodies. Nat Methods 9, 582584.

Rizzo, M.A., Davidson, M. W. and Piston, D. W. (2010). Fluorescent Protein Tracking and Detection: Applications Using Fluorescent Proteins in Living Cells. In Live Cell Imaging: A Laboratory Manual, R.D. Goldman, ed. (Cold Spring Harbor, NY, USA: CSHL Press), pp. 113.

Rouet, P., Smih, F., and Jasin, M. (1994). Expression of a site-specific endonuclease stimulates homologous recombination in mammalian cells. Proc Natl Acad Sci U S A 91, 6064-6068.

Rust, M.J., Bates, M., and Zhuang, X. (2006). Sub-diffraction-limit imaging by stochastic optical reconstruction microscopy (STORM). Nat Methods 3, 793-795.

Samsonov, A., Zenser, N., Zhang, F., Zhang, H., Fetter, J., and Malkov, D. (2013). Tagging of genomic STAT3 and STAT1 with fluorescent proteins and insertion of a luciferase reporter in the cyclin D1 gene provides a modified A549 cell line to screen for selective STAT3 inhibitors. PLoS One 8, e68391.

Sander, J.D., Dahlborg, E.J., Goodwin, M.J., Cade, L., Zhang, F., Cifuentes, D., Curtin, S.J., Blackburn, J.S., Thibodeau-Beganny, S., Qi, Y., et al. (2011). Selection-free zinc-fingernuclease engineering by context-dependent assembly (CoDA). Nat Methods 8, 67-69.

Sander, J.D., and Joung, J.K. (2014). CRISPR-Cas systems for editing, regulating and targeting genomes. Nat Biotechnol. 
Sanger, F., Nicklen, S., and Coulson, A.R. (1977). DNA sequencing with chain-terminating inhibitors. Proc Natl Acad Sci U S A 74, 5463-5467.

Sanjana, N.E., Cong, L., Zhou, Y., Cunniff, M.M., Feng, G., and Zhang, F. (2012). A transcription activator-like effector toolbox for genome engineering. Nat Protoc 7, 171-192.

Santel, A., Frank, S., Gaume, B., Herrler, M., Youle, R.J., and Fuller, M.T. (2003). Mitofusin-1 protein is a generally expressed mediator of mitochondrial fusion in mammalian cells. J Cell Sci 116, 2763-2774.

Schleicher, M., Shepherd, B.R., Suarez, Y., Fernandez-Hernando, C., Yu, J., Pan, Y., Acevedo, L.M., Shadel, G.S., and Sessa, W.C. (2008). Prohibitin-1 maintains the angiogenic capacity of endothelial cells by regulating mitochondrial function and senescence. $\mathrm{J}$ Cell Biol $180,101-112$.

Schneeberger, M., Dietrich, M.O., Sebastian, D., Imbernon, M., Castano, C., Garcia, A., Esteban, Y., Gonzalez-Franquesa, A., Rodriguez, I.C., Bortolozzi, A., et al. (2013). Mitofusin 2 in POMC neurons connects ER stress with leptin resistance and energy imbalance. Cell $155,172-187$.

Shah, S.A., Erdmann, S., Mojica, F.J., and Garrett, R.A. (2013). Protospacer recognition motifs: mixed identities and functional diversity. RNA Biol 10, 891-899.

Shalem, O., Sanjana, N.E., Hartenian, E., Shi, X., Scott, D.A., Mikkelsen, T.S., Heckl, D., Ebert, B.L., Root, D.E., Doench, J.G., et al. (2014). Genome-scale CRISPR-Cas9 knockout screening in human cells. Science $343,84-87$.

Shaner, N.C., Steinbach, P.A., and Tsien, R.Y. (2005). A guide to choosing fluorescent proteins. Nat Methods 2, 905-909.

Sharma, A., and Qadri, A. (2004). Vi polysaccharide of Salmonella typhi targets the prohibitin family of molecules in intestinal epithelial cells and suppresses early inflammatory responses. Proc Natl Acad Sci U S A 101, 17492-17497.

Shimomura, O., Johnson, F.H., and Saiga, Y. (1962). Extraction, purification and properties of aequorin, a bioluminescent protein from the luminous hydromedusan, Aequorea. $\mathrm{J}$ Cell Comp Physiol 59, 223-239.

Shroff, H., Galbraith, C.G., Galbraith, J.A., White, H., Gillette, J., Olenych, S., Davidson, M.W., and Betzig, E. (2007). Dual-color superresolution imaging of genetically expressed probes within individual adhesion complexes. Proc Natl Acad Sci U S A 104, 20308-20313.

Sjostrand, F.S. (1953). Electron microscopy of mitochondria and cytoplasmic double membranes. Nature 171, 30-32.

Smirnova, E., Griparic, L., Shurland, D.L., and van der Bliek, A.M. (2001). Dynamin-related protein Drp1 is required for mitochondrial division in mammalian cells. Mol Biol Cell 12, 22452256.

Smith, G.R. (1994). Hotspots of homologous recombination. Experientia 50, 234-241. 
Smith, P.K., Krohn, R.I., Hermanson, G.T., Mallia, A.K., Gartner, F.H., Provenzano, M.D., Fujimoto, E.K., Goeke, N.M., Olson, B.J., and Klenk, D.C. (1985). Measurement of protein using bicinchoninic acid. Anal Biochem 150, 76-85.

Smithies, O., Gregg, R.G., Boggs, S.S., Koralewski, M.A., and Kucherlapati, R.S. (1985). Insertion of DNA sequences into the human chromosomal beta-globin locus by homologous recombination. Nature 317, 230-234.

Snapp, E. (2005). Design and use of fluorescent fusion proteins in cell biology. Curr Protoc Cell Biol Chapter 21, Unit 2124.

Steglich, G., Neupert, W., and Langer, T. (1999). Prohibitins regulate membrane protein degradation by the m-AAA protease in mitochondria. Mol Cell Biol 19, 3435-3442.

Sternberg, S.H., Redding, S., Jinek, M., Greene, E.C., and Doudna, J.A. (2014). DNA interrogation by the CRISPR RNA-guided endonuclease Cas9. Nature 507, 62-67.

Suen, D.F., Norris, K.L., and Youle, R.J. (2008). Mitochondrial dynamics and apoptosis. Genes Dev 22, 1577-1590.

Sukhorukov, V.M., Dikov, D., Busch, K., Strecker, V., Wittig, I., and Bereiter-Hahn, J. (2010). Determination of protein mobility in mitochondrial membranes of living cells. Biochimica et Biophysica Acta (BBA) - Biomembranes 1798, 2022-2032.

Sun, L., Liu, L., Yang, X.J., and Wu, Z. (2004). Akt binds prohibitin 2 and relieves its repression of MyoD and muscle differentiation. J Cell Sci 117, 3021-3029.

Tang, T.H., Bachellerie, J.P., Rozhdestvensky, T., Bortolin, M.L., Huber, H., Drungowski, M., Elge, T., Brosius, J., and Huttenhofer, A. (2002). Identification of 86 candidates for small nonmessenger RNAs from the archaeon Archaeoglobus fulgidus. Proc Natl Acad Sci U S A 99, 7536-7541.

Tatsuta, T., Model, K., and Langer, T. (2005). Formation of membrane-bound ring complexes by prohibitins in mitochondria. Mol Biol Cell 16, 248-259.

Terashima, M., Kim, K.M., Adachi, T., Nielsen, P.J., Reth, M., Kohler, G., and Lamers, M.C. (1994). The IgM antigen receptor of $B$ lymphocytes is associated with prohibitin and a prohibitin-related protein. EMBO J 13, 3782-3792.

Terpe, K. (2003). Overview of tag protein fusions: from molecular and biochemical fundamentals to commercial systems. Appl Microbiol Biotechnol 60, 523-533.

Testa, I., D'Este, E., Urban, N.T., Balzarotti, F., and Hell, S.W. (2015). Dual channel RESOLFT nanoscopy by using fluorescent state kinetics. Nano Lett 15, 103-106.

Testa, I., Urban, N.T., Jakobs, S., Eggeling, C., Willig, K.I., and Hell, S.W. (2012). Nanoscopy of living brain slices with low light levels. Neuron 75, 992-1000.

Thakore, P.I., D'Ippolito, A.M., Song, L., Safi, A., Shivakumar, N.K., Kabadi, A.M., Reddy, T.E., Crawford, G.E., and Gersbach, C.A. (2015). Highly specific epigenome editing by CRISPR-Cas9 repressors for silencing of distal regulatory elements. Nat Methods. 
Thomas, K.R., and Capecchi, M.R. (1986). Introduction of homologous DNA sequences into mammalian cells induces mutations in the cognate gene. Nature 324, 34-38.

Tojkander, S., Gateva, G., and Lappalainen, P. (2012). Actin stress fibers--assembly, dynamics and biological roles. J Cell Sci 125, 1855-1864.

Tol, M., Akar, A.R., Durdu, S., Ayyildiz, E., and Ilhan, O. (2008). Comparison of different needle diameters and flow rates on bone marrow mononuclear stem cell viability: an ex vivo experimental study. Cytotherapy 10, 98-99.

Tondera, D., Grandemange, S., Jourdain, A., Karbowski, M., Mattenberger, Y., Herzig, S., Da Cruz, S., Clerc, P., Raschke, I., Merkwirth, C., et al. (2009). SLP-2 is required for stressinduced mitochondrial hyperfusion. The EMBO Journal 28, 1589-1600.

Tsai, S.Q., Wyvekens, N., Khayter, C., Foden, J.A., Thapar, V., Reyon, D., Goodwin, M.J., Aryee, M.J., and Joung, J.K. (2014). Dimeric CRISPR RNA-guided FokI nucleases for highly specific genome editing. Nat Biotechnol 32, 569-576.

Tsai, S.Q., Zheng, Z., Nguyen, N.T., Liebers, M., Topkar, V.V., Thapar, V., Wyvekens, N., Khayter, C., lafrate, A.J., Le, L.P., et al. (2015). GUIDE-seq enables genome-wide profiling of off-target cleavage by CRISPR-Cas nucleases. Nat Biotechnol 33, 187-197.

Ulbrich, M.H. (2015). Counting Molecules: Toward Quantitative Imaging. In Far-Field Optical Nanoscopy, P. Tinnefeld, C. Eggeling, and S.W. Hell, eds. (Heidelberg: Springer), pp. 263291.

Urnov, F.D., Rebar, E.J., Holmes, M.C., Zhang, H.S., and Gregory, P.D. (2010). Genome editing with engineered zinc finger nucleases. Nat Rev Genet 11, 636-646.

Vogel, F., Bornhovd, C., Neupert, W., and Reichert, A.S. (2006). Dynamic subcompartmentalization of the mitochondrial inner membrane. J Cell Biol 175, 237-247.

Vouillot, L., Thelie, A., and Pollet, N. (2015). Comparison of T7E1 and surveyor mismatch cleavage assays to detect mutations triggered by engineered nucleases. G3 (Bethesda) 5, 407-415.

Wang, T., Wei, J.J., Sabatini, D.M., and Lander, E.S. (2014a). Genetic screens in human cells using the CRISPR-Cas9 system. Science 343, 80-84.

Wang, Y.J., Guo, X.L., Li, S.A., Zhao, Y.Q., Liu, Z.C., Lee, W.H., Xiang, Y., and Zhang, Y. (2014b). Prohibitin is involved in the activated internalization and degradation of proteaseactivated receptor 1. Biochim Biophys Acta 1843, 1393-1401.

Wang, X., Wang, Y., Wu, X., Wang, J., Wang, Y., Qiu, Z., Chang, T., Huang, H., Lin, R.J., and Yee, J.K. (2015). Unbiased detection of off-target cleavage by CRISPR-Cas9 and TALENs using integrase-defective lentiviral vectors. Nat Biotechnol 33, 175-178.

Westermann, B. (2010). Mitochondrial fusion and fission in cell life and death. Nat Rev Mol Cell Biol 11, 872-884. 
Wright, D.A., Thibodeau-Beganny, S., Sander, J.D., Winfrey, R.J., Hirsh, A.S., Eichtinger, M., Fu, F., Porteus, M.H., Dobbs, D., Voytas, D.F., et al. (2006). Standardized reagents and protocols for engineering zinc finger nucleases by modular assembly. Nat Protocols 1, 16371652.

Wu, J.-Q., and Pollard, T.D. (2005). Counting Cytokinesis Proteins Globally and Locally in Fission Yeast. Science 310, 310-314.

Wu, S., Ying, G., Wu, Q., and Capecchi, M.R. (2008). A protocol for constructing gene targeting vectors: generating knockout mice for the cadherin family and beyond. Nat Protoc 3 , 1056-1076.

Wu, X., Kriz, A.J., and Sharp, P.A. (2014a). Target specificity of the CRISPR-Cas9 system. Quant Biol 2, 59-70.

Wu, X., Scott, D.A., Kriz, A.J., Chiu, A.C., Hsu, P.D., Dadon, D.B., Cheng, A.W., Trevino, A.E., Konermann, S., Chen, S., et al. (2014b). Genome-wide binding of the CRISPR endonuclease Cas9 in mammalian cells. Nat Biotechnol 32, 670-676.

Wyvekens, N., Topkar, V.V., Khayter, C., Joung, J.K., and Tsai, S.Q. (2015). Dimeric CRISPR RNA-Guided Fokl-dCas9 Nucleases Directed by Truncated gRNAs for Highly Specific Genome Editing. Hum Gene Ther 26, 425-431.

Yang, F., Moss, L.G., and Phillips, G.N., Jr. (1996). The molecular structure of green fluorescent protein. Nat Biotechnol 14, 1246-1251.

Young, J.C., Barral, J.M., and Ulrich Hartl, F. (2003). More than folding: localized functions of cytosolic chaperones. Trends Biochem Sci 28, 541-547.

Youle, R.J., and van der Bliek, A.M. (2012). Mitochondrial fission, fusion, and stress. Science 337, 1062-1065.

Zetsche, B., Volz, S.E., and Zhang, F. (2015). A split-Cas9 architecture for inducible genome editing and transcription modulation. Nat Biotechnol 33, 139-142.

Zhang, C.C., and Shapiro, D.J. (2000). Activation of the p38 mitogen-activated protein kinase pathway by estrogen or by 4-hydroxytamoxifen is coupled to estrogen receptor-induced apoptosis. J Biol Chem 275, 479-486.

Zick, M., Rabl, R., and Reichert, A.S. (2009). Cristae formation-linking ultrastructure and function of mitochondria. Biochimica et Biophysica Acta (BBA) - Molecular Cell Research 1793, 5-19. 


\section{Supplementary information}

Table 4: Primers used for one-step cloning of overexpression plasmid. All constructs are based on the pFLAG-CMV-5.1 backbone.

\begin{tabular}{|c|c|c|}
\hline Plasmid name & Primer & Sequence (5' to $\left.3^{\prime}\right)$ \\
\hline \multirow{4}{*}{$\begin{array}{l}\text { VIM- } \\
\text { rsEGFP2 }\end{array}$} & MR843_VIM_F & $\begin{array}{l}\text { TTCATCGATAGATCTGATGCCACCATGTC } \\
\text { CACCAGGTCCGTGTC }\end{array}$ \\
\hline & MR844_VIM_R & $\begin{array}{l}\text { GAACCACTACCGGCGGCCGCTTCAAGGT } \\
\text { CATCGTGATGCTGAG }\end{array}$ \\
\hline & MR845_rsEGFP2_F & $\begin{array}{l}\text { CCGCCGGTAGTGGTTCAGGGGTGAGCAA } \\
\text { GGGCGAGGAGC }\end{array}$ \\
\hline & MR846_rsEGFP2_R & $\begin{array}{l}\text { GTCGACTGGTACCGATTTACTTGTACAGC } \\
\text { TCGTCCATGCC }\end{array}$ \\
\hline \multirow{4}{*}{$\begin{array}{l}\text { ZYX- } \\
\text { rsEGFP2 }\end{array}$} & MR930_ZYX_F & $\begin{array}{l}\text { TTCATCGATAGATCTGATGCCACCATGGC } \\
\text { GGCCCCCCGCCCGTCTC }\end{array}$ \\
\hline & MR931_ZYX_R & $\begin{array}{l}\text { CCGGTGCCGCTGCCGGTCTGGGCTCTAG } \\
\text { CAGTGTGGCACTTCCG }\end{array}$ \\
\hline & MR932_rsEGFP2_F & $\begin{array}{l}\text { CCGGCAGCGGCACCGGTGTGAGCAAGG } \\
\text { GCGAGGAGCTG }\end{array}$ \\
\hline & MR933_sEGFP2_R & $\begin{array}{l}\text { GTCGACTGGTACCGATTTACTTGTACAGC } \\
\text { TCGTCCATGCCGAG }\end{array}$ \\
\hline \multirow{4}{*}{$\begin{array}{l}\text { HMGA1- } \\
\text { rsEGFP2 }\end{array}$} & MR956_HMGA1_F & $\begin{array}{l}\text { TTCATCGATAGATCTGATGCCACCATGAG } \\
\text { TGAGTCGAGCTCGAAGTCC }\end{array}$ \\
\hline & MR957_HMGA1_R & $\begin{array}{l}\text { CGGATCCGCTGCCCTGCTCCTCCTCCGA } \\
\text { GGACT }\end{array}$ \\
\hline & MR958_rsEGFP2_F & $\begin{array}{l}\text { CAGGGCAGCGGATCCGGCGTGAGCAAG } \\
\text { GGCGAGGAGCTG }\end{array}$ \\
\hline & MR959_rsEGFP2_R & $\begin{array}{l}\text { GTCGACTGGTACCGATGGTACCTTACTTG } \\
\text { TACAGCTCGTCCATGCCGAG }\end{array}$ \\
\hline \multirow{4}{*}{ PHB1-DK } & MR1143_PHB1_F & $\begin{array}{l}\text { TTCATCGATAGATCTGATGCCACCATGGC } \\
\text { TGCCAAAGTGTTTGAGTCC }\end{array}$ \\
\hline & MR1144_PHB1_R & $\begin{array}{l}\text { CCACTACCCTGGGGCAGCTGGAGGAGCA } \\
\mathrm{C}\end{array}$ \\
\hline & MR1145_DK_F & $\begin{array}{l}\text { TGCCCCAGGGTAGTGGTTCAGGGGTGAG } \\
\text { CAAGGGCGAGGAGCTG }\end{array}$ \\
\hline & MR1146_DK_R & $\begin{array}{l}\text { GTCGACTGGTACCGATTTACTTGTACAGC } \\
\text { TCGTCCATG }\end{array}$ \\
\hline \multirow{4}{*}{ PHB2-DK } & MR1147_PHB2_F & $\begin{array}{l}\text { TTCATCGATAGATCTGATATGGCCCAGAA } \\
\text { CTTGAAGGACTTG }\end{array}$ \\
\hline & MR1148_PHB2_R & $\begin{array}{l}\text { CCACTACCTTTCTTACCCTTGATGAGGCT } \\
\text { GTC }\end{array}$ \\
\hline & MR1145_DK_F & $\begin{array}{l}\text { TGCCCCAGGGTAGTGGTTCAGGGGTGAG } \\
\text { CAAGGGCGAGGAGCTG }\end{array}$ \\
\hline & MR1149_DK_R & $\begin{array}{l}\text { GGTAAGAAAGGTAGTGGTTCAGGGGTGA } \\
\text { GCAAGGGCGAGGAGCTG }\end{array}$ \\
\hline \multirow{2}{*}{ ESR1 } & MR1216_ESR1_F & $\begin{array}{l}\text { TTCATCGATAGATCTGATGCCACCATGAC } \\
\text { CATGACCCTCCACACC }\end{array}$ \\
\hline & MR1217_ESR1_R & $\begin{array}{l}\text { GTCGACTGGTACCGATTTAGACCGTGGCA } \\
\text { GGGAAAC }\end{array}$ \\
\hline
\end{tabular}


Table 5: Primers used for donor plasmid cloning. All donor plasmids are based on the pUC57 backbone. Note: 'ZYX gBlock' is a double-stranded DNA fragment and the given sequence corresponds to the ' 5 ' to 3 ' sequence of the plus strand.

\begin{tabular}{|c|c|c|}
\hline Donor plasmid & Primer name & Sequence (5' to 3') \\
\hline \multirow{6}{*}{$\begin{array}{l}\text { HMGA1- } \\
\text { rsEGFP2 }\end{array}$} & GA145_LHA_F & $\begin{array}{l}\text { TCTCGCGAATGCATCTAGATGATGTGACCCACC } \\
\text { ACACTGCACTGG }\end{array}$ \\
\hline & GA146_LHA_R & $\begin{array}{l}\text { ATCCGCTGCCCTGCTCCTCCTCCGAGGACTCCT } \\
\text { GC }\end{array}$ \\
\hline & GA146_rsEGFP2_F & $\begin{array}{l}\text { GGAGGAGCAGGGCAGCGGATCCGGCGTGAGC } \\
\text { AAGGGCGAGGAGC }\end{array}$ \\
\hline & GA147_rsEGFP2_R & $\begin{array}{l}\text { CACGCATGGGGGTACCTTACTTGTACAGCTCGT } \\
\text { CCATG }\end{array}$ \\
\hline & GA148_RHA_F & $\begin{array}{l}\text { GTAAGGTACCCCCATGCGTGCCGCCTGCTCCT } \\
\text { CAC }\end{array}$ \\
\hline & GA149_RHA_R & $\begin{array}{l}\text { AGGCCTCTGCAGTCGACGATGTGAGCTCCTTGT } \\
\text { TATTGGTGCCCCATCTG }\end{array}$ \\
\hline \multirow{6}{*}{$\begin{array}{l}\text { VIM- } \\
\text { rsEGFP2 }\end{array}$} & MR449_LHA_F & $\begin{array}{l}\text { ATATTAGGTACCCCTTAAACCACTTCCTAAAGCA } \\
\text { GCTACATGAAACAGCTTCACTAGACTACC }\end{array}$ \\
\hline & MR450_LHA_R & $\begin{array}{l}\text { TATATTGCGGCCGCTTCAAGGTCATCGTGATGC } \\
\text { TGAGAAGTTTCGTTGATAACC }\end{array}$ \\
\hline & MR451_rsEGFP2_F & $\begin{array}{l}\text { ATATTAGCGGCCGCCGGTAGTGGTTCAGGGGT } \\
\text { GAGCAAGGGCGAGGAGCTGTTCACCG }\end{array}$ \\
\hline & MR452_rsEGFP2_R & $\begin{array}{l}\text { TTAATTCCATGGTTACTTGTACAGCTCGTCCATG } \\
\text { CCGAGAGTGATCCC }\end{array}$ \\
\hline & MR453_RHA_F & $\begin{array}{l}\text { CGAAACTTCTCAGCATCACGATGACCTTCCATG } \\
\text { GAAATTGCACACACTCAGTG }\end{array}$ \\
\hline & MR454_RHA_R & $\begin{array}{l}\text { TATTTAGTCGACGTTATGATTAAAATCACAGACC } \\
\text { TGAGCTCATTTTAGAGAGACCTATCTTC }\end{array}$ \\
\hline \multirow{7}{*}{$\begin{array}{l}\text { ZYX- } \\
\text { rsEGFP2 }\end{array}$} & GA403_LHA_F & $\begin{array}{l}\text { TCTCGCGAATGCATCTAGATGAAAGCCCTGGCT } \\
\text { AACTCGGCTGGC }\end{array}$ \\
\hline & GA404_LHA_R & TACCCCACCCCATCCAGCTCCATCTTG \\
\hline & GA405_rsEGFP2_F & $\begin{array}{l}\text { GGCAGCGGCACCGGTGTGAGCAAGGGCGAGG } \\
\text { AGC }\end{array}$ \\
\hline & GA406_rsEGFP2_R & $\begin{array}{l}\text { CTGTCCTCACGCTAGCTTACTTGTACAGCTCGT } \\
\text { CCATG }\end{array}$ \\
\hline & GA407_RHA_F & $\begin{array}{l}\text { GTAAGCTAGCGTGAGGACAGGCCCTCTTCAGA } \\
\text { CCG }\end{array}$ \\
\hline & GA408_RHA_R & $\begin{array}{l}\text { AGGCCTCTGCAGTCGACGATGACGCAGATGGG } \\
\text { AATCACACTGCCC }\end{array}$ \\
\hline & ZYX_gBlock & $\begin{array}{l}\text { GAGCTGGATGGGGTGGGGTAGGGTGGAGCAG } \\
\text { AGCAGGGGCCTTCCGGTCCAGTGCCCCTCACC } \\
\text { CTTCCTTCTTCCCAGGACTGCGGGAAGCCTCTG } \\
\text { TCAATTGAGGCTGATGACAATGGCTGCTTCCCT } \\
\text { CTGGAAGGTCATGTGCTCTGTCGGAAGTGCCA } \\
\text { CACTGCTAGAGCCCAGACCGGCAGCGGCACCG } \\
\text { GTGTGAG }\end{array}$ \\
\hline \multirow{4}{*}{ PHB1-DK } & GA265_LHA_F & $\begin{array}{l}\text { TCTCGCGAATGCATCTAGATAGGTCACACGTTG } \\
\text { CAGAGAGCTGTCTTCC }\end{array}$ \\
\hline & GA266_LHA_R & $\begin{array}{l}\text { ATCCGCTGCCCTGGGGCAGCTGGAGGAGCACG } \\
\text { GAC }\end{array}$ \\
\hline & GA267_DK_F & $\begin{array}{l}\text { GCTGCCCCAGGGCAGCGGATCCGGCGTGAGC } \\
\text { AAGGGCGAGGAGC }\end{array}$ \\
\hline & GA268_DK_R & $\begin{array}{l}\text { GGGTGGGCCCGGTACCTTACTTGTACAGCTCG } \\
\text { TCCATG }\end{array}$ \\
\hline
\end{tabular}




\begin{tabular}{lll}
\hline & GA269_RHA_F & $\begin{array}{l}\text { GTAAGGTACCGGGCCCACCCTGCCTGCACCTC } \\
\text { CGC }\end{array}$ \\
\cline { 2 - 3 } & GA270_RHA_R & $\begin{array}{l}\text { AGGCCTCTGCAGTCGACGATCCAGGAACGTAG } \\
\text { GTCGGACACGTCTTTGGC }\end{array}$ \\
\hline GA271_LHA_F & $\begin{array}{l}\text { TCTCGCGAATGCATCTAGATATGTGTTACTCATT } \\
\text { GCTGCACCCCT }\end{array}$ \\
\cline { 2 - 3 } PHB2-DK & GA272_LHA_R & $\begin{array}{l}\text { ATCCGCTGCCTTTCTTACCCTTGATGAGGCTGT } \\
\text { CAC }\end{array}$ \\
\cline { 2 - 3 } & GA273_DK_F & $\begin{array}{l}\text { GGGTAAGAAAGGCAGCGGATCCGGCGTGAGCA } \\
\text { AGGGCGAGGAGC }\end{array}$ \\
\cline { 2 - 3 } & GA274_DK_R & $\begin{array}{l}\text { GTGACTATGCGGTACCTTACTTGTACAGCTCGT } \\
\text { CCATG }\end{array}$ \\
\cline { 2 - 3 } & GA275_RHA_F & $\begin{array}{l}\text { GTAAGGTACCGCATAGTCACCAAGGACTCCACC } \\
\text { CC }\end{array}$ \\
\cline { 2 - 3 } & GA276_RHA_R & $\begin{array}{l}\text { AGGCCTCTGCAGTCGACGATGGTGCCTGAGAT } \\
\text { TCGGGAAGGCCTG }\end{array}$ \\
\hline PHB1-DK-res & MR1038_SDM & $\begin{array}{l}\text { GCACCTCCGCGGGCTGACTAAACCACAGCCCC } \\
\text { GGGGCTGTGGTTTAGTCAGCCCGCGGAGGTGC }\end{array}$ \\
\cline { 2 - 3 } PHB2-DK-res & MR1039_SDM & $\begin{array}{l}\text { CAGAGGAAGTGGATCTGCTTCTTTAGTTTTTGA } \\
\text { GGAGCCA }\end{array}$ \\
\cline { 2 - 3 } & MR1040_SDM & $\begin{array}{l}\text { TGGCTCCTCAAAAACTAAAGAAGCAGATCCACT } \\
\text { TCCTCTG }\end{array}$ \\
\hline
\end{tabular}

Table 6: Primers used for gRNA cloning.

\begin{tabular}{|c|c|c|c|}
\hline Gene & gRNA & Primer & Sequence (5' to 3') \\
\hline \multirow{4}{*}{ HMGA1 } & \multirow{2}{*}{ gRNA1 } & MR577_F & CACCGAGGAGCAGGCGGCACGCAT \\
\hline & & MR578_R & AAACATGCGTGCCGCCTGCTCCTC \\
\hline & \multirow{2}{*}{ gRNA2 } & MR579_F & CACCGCCAACAACTGCCCACCTCAC \\
\hline & & MR560_R & AAACGTGAGGTGGGCAGTTGTTGGC \\
\hline \multirow{4}{*}{ VIM } & \multirow{2}{*}{ gRNA1 } & MR292_F & CACCGCGCAAGATAGATTTGGAAT \\
\hline & & MR293_R & AAACATTCCAAATCTATCTTGCGC \\
\hline & \multirow{2}{*}{ gRNA2 } & MR626_F & CACCGTCAGGAGCGCAAGATAGATT \\
\hline & & MR627_R & AAACAATCTATCTTGCGCTCCTGAC \\
\hline \multirow{4}{*}{ ZYX } & \multirow{2}{*}{ gRNA1 } & MR529_F & CACCGACAGAGCACGTGACCGTCC \\
\hline & & MR530_R & AAACGGACGGTCACGTGCTCTGTC \\
\hline & \multirow{2}{*}{ gRNA2 } & MR531_F & CACCGTGTCATCTGCCTCAATCGAC \\
\hline & & MR532_R & AAACGTCGATTGAGGCAGATGACAC \\
\hline \multirow{4}{*}{ PHB1 } & \multirow{2}{*}{ gRNA1 } & MR714_F & CACCGTGCACCTCCGCGGGCTGACT \\
\hline & & MR715_R & AAACAGTCAGCCCGCGGAGGTGCAC \\
\hline & \multirow{2}{*}{ gRNA2 } & MR716_F & CACCGCTGCACCTCCGCGGGCTGAC \\
\hline & & MR717_R & AAACGTCAGCCCGCGGAGGTGCAGC \\
\hline \multirow{4}{*}{ PHB2 } & \multirow{2}{*}{ gRNA1 } & MR718_F & CACCGGGTGGAGTTCTTGGTGACT \\
\hline & & MR719_R & AAACAGTCACCAAGAACTCCACCC \\
\hline & \multirow{2}{*}{ gRNA2 } & MR720_F & CACCGCTGGCTGGCTCCTCAAAAAC \\
\hline & & MR721_R & AAACGTTTTTGAGGAGCCAGCCAGC \\
\hline
\end{tabular}


Table 7: Plasmid templates and primers used to generate T7-gRNA templates for IVT of gRNAs. The T7 promoter sequence (bold) was introduced via the respective forward primer. The reverse primer was universal for the guide RNA scaffold.

\begin{tabular}{|c|c|c|c|}
\hline Template & gRNA & Primer & Sequence (5' to $\left.3^{\prime}\right)$ \\
\hline pMR127 & PHB1-gRNA1 & MR1172_F & $\begin{array}{l}\text { TTAATACGACTCACTATAGGTGCACCTCCGC } \\
\text { GGGCTGACTGTTTTAGAG }\end{array}$ \\
\hline pMR128 & PHB1-gRNA2 & MR1173_F & $\begin{array}{l}\text { TTAATACGACTCACTATAGGCTGCACCTCCG } \\
\text { CGGGCTGACGT }\end{array}$ \\
\hline pMR199 & PHB1-gRNA3 & MR1174_F & $\begin{array}{l}\text { TTAATACGACTCACTATAGGAGCCCGCGGA } \\
\text { GGTGCAGGCAGT }\end{array}$ \\
\hline pMR129 & PHB2-gRNA1 & MR1178_F & $\begin{array}{l}\text { TTAATACGACTCACTATAGGGGTGGAGTTCT } \\
\text { TGGTGACTGTTTTAGAG }\end{array}$ \\
\hline pMR130 & PHB2-gRNA2 & MR1179_F & $\begin{array}{l}\text { TTAATACGACTCACTATAGGCTGGCTGGCTC } \\
\text { CTCAAAAACGT }\end{array}$ \\
\hline pMR208 & PHB2-gRNA3 & MR1180_F & $\begin{array}{l}\text { TTAATACGACTCACTATAGGCAGGGTAGGA } \\
\text { GGGTCGTAGAGTTTTAGAGCT }\end{array}$ \\
\hline Universal & Universal & MR1013_R & AAAAGCACCGACTCGGTGCC \\
\hline
\end{tabular}

Table 8: Primers used for amplification of DNA substrates to test gRNAs in vitro.

\begin{tabular}{lll}
\hline Amplicon & Primer & Sequence (5' to 3') \\
\hline \multirow{2}{*}{ PHB1 } & MR900_F & CTTTCTGGTGAAGGGCCTTTGGTTGTAGC \\
\cline { 2 - 3 } & MR901_R & GGTAGGGAGGTGGATAAAAAAGTAGATGGATG \\
\hline \multirow{2}{*}{ PHB2 } & MR902_F & GCTGTTGTTGCTTCTGTGGGGTCCTGC \\
\cline { 2 - 3 } & MR903_R & GCTTCCCCTTAGCCGCCCCAGCCAGATTC \\
\hline
\end{tabular}

Table 9: Primers used for amplification of DNA substrates for T7 Endonuclease I assay.

\begin{tabular}{lll}
\hline Amplicon & Primer & Sequence (5' to 3') \\
\hline \multirow{2}{*}{ HMGA1 } & MR962_F & CAAGTTGTTTTGGAGTTTGTGCCTGG \\
\cline { 2 - 3 } & MR897_R & GCTGGTGTGCTGTGTAGTGTGGTGGTGAGG \\
\hline \multirow{2}{*}{ ZYX } & MR926_F & GTTGGGTGTGGCTGGAAAAAGCGATGAC \\
\cline { 2 - 3 } & MR927_R & GGATGCTAGGGAGGGGTTGGAAATGGGAG \\
\hline
\end{tabular}

Table 10: Primers used for on-target site analysis via PCR and DNA sequencing.

\begin{tabular}{lll}
\hline Gene & Primer & Sequence (5' to 3') \\
\hline \multirow{2}{*}{ HMGA1 } & MR952_out_F & GTCCCTCCCTCTCCTGCTCCTAGAATACTCAG \\
& MR953_out_R & CAATGACGGATGTCGAAGAATGGAACATTGAAC \\
& MR834_GFP_R & CACGCTGCCGTCCTCGATGTTG \\
VIM & MR847_out_F & CTAATCTGGATTCACTCCCTCTGGTTGATACC \\
& MR849_out_R & CCTATATCTTGGCAACTTCCTATGCTTTAACTCCC \\
& MR834_GFP_R & CACGCTGCCGTCCTCGATGTTG \\
ZYX & MR928_out_F & CTTGCTTTGGGAGAGTGACTGGTGAGGC \\
& MR929_out_R & GCTGAGTAAGGAGCTGGAGCTGTCTGGGC \\
& MR834_GFP_R & CACGCTGCCGTCCTCGATGTTG \\
& MR1269_in_F & CCTGCAGCCAACAGAGATGTATCCCTCC \\
PHB1 & MR1051_out_R & GCAGTTTATACACATTTGTTTCCTTCCCAG \\
& MR1050_out_F & GCTAAATCCATCCAGATGTGTCAAAGAATG \\
& MR501_DK_R & GCACGACTTCTTCAAGTCCGCCATGCC \\
PHB2 & MR1052_out_F & TTCCCTGACCTTTTGTTCTAATCATAGCTG \\
& MR1053_out_R & TCTAGTTGTTTATCTCATCTTAGCCTCCCA \\
& MR501_DK_R & GCACGACTTCTTCAAGTCCGCCATGCC \\
\hline \multirow{3}{*}{} & &
\end{tabular}


Table 11: Primers used for off-target site analysis via DNA sequencing.

\begin{tabular}{|c|c|c|}
\hline gRNA & Primer & Sequence (5' to $\left.3^{\prime}\right)$ \\
\hline \multirow{4}{*}{ HMGA1-gRNA1 } & MR1014_OT1_F & CAGGGTCGTGTGATGAATTCATTTGGTTATC \\
\hline & MR1015_OT1_R & GCATTATATCAGCCTACGGTGGAGAAGTGG \\
\hline & MR1016_OT2_F & GATAGGCCCGGAGAATTTGCTCAGGTTC \\
\hline & MR1017_OT2_R & GTATACTGGGAGATGATATCCTGCACCCTCACTG \\
\hline \multirow{4}{*}{ HMGA1-gRNA2 } & MR1018_OT1_F & GTTTAGCTGGGATTAGAGCCACAGACCTTG \\
\hline & MR1019_OT1_R & GTTTGGAGTCTCACTGCTGCTACTGTCTCATC \\
\hline & MR1020_OT2_F & GAAAGAGTATGGGAGAGATAGCGTCCTTCTAG \\
\hline & MR1021_OT2_R & GATGTCAGTTATGCCAATAATAAATTAATTAAGGGC \\
\hline \multirow{4}{*}{ VIM-gRNA1 } & MR1022_OT1_F & GCAGAGGCAAATTTCAACTAGTCAAACCC \\
\hline & MR1023_OT1_R & GTCACACTTAGATTTATCCTACAAGCTGCCC \\
\hline & MR1024_OT2_F & GACATAATAATACAGACATTTGTGGGTGGTAGG \\
\hline & MR1025_OT2_R & CTTTACTTAGAATAATGGCCTCCAGCTCTATCC \\
\hline \multirow{4}{*}{ VIM-gRNA2 } & MR1026_OT1_F & GGGGTTAGTTACTCAGTCACAATTGATGAAGGG \\
\hline & MR1027_OT1_R & CCTGCGTTAACAGTGAGTTGGAGAAACACTAG \\
\hline & MR1028_OT2_F & CAATAATTTGCCCTTTTGCTTCCACTGAG \\
\hline & MR1029_OT2_R & GTAATCTCACTCCATTACTTTTAGAATGTCAAGCCC \\
\hline \multirow{4}{*}{ ZYX-gRNA1 } & MR1030_OT1_F & GCCCGGCCTAATTTGTGTATTTTTAGTAGAG \\
\hline & MR1031_OT1_R & GTAGAGACGGTGTTTTACCATGTTAACCAGG \\
\hline & MR1032_OT2_F & GAGAGCTCGGGTTGCTGTTTCTACTTCAG \\
\hline & MR1033_OT2_R & CGTTATAGTCCTCGAAGATAATTCTAGGTGGGAG \\
\hline \multirow{6}{*}{ PHB1-gRNA2 } & MR1364_OT1_F & GAGATGGGGTCTCATCATGTTGCTCAGAC \\
\hline & MR1365_OT1_R & CACAGAAGCAGTGGAAGCCAAACAGGTG \\
\hline & MR1366_OT2_F & CACTTCGAACTGATGAGTCCTCAAACTGTAACC \\
\hline & MR1367_OT2_R & GAAGGAGTTCACAGAAGCGATGGAAGCC \\
\hline & MR1368_OT3_F & CGGGAAGGAGTTCACAGAAGCGGTGG \\
\hline & MR1369_OT3_R & GCATGTGTCCTCATCCGCAAATCTTCCATC \\
\hline \multirow{6}{*}{ PHB2-gRNA2 } & MR1370_OT1_F & CACAACTTGGTTTAGAATAAAGCCTCCACAGAC \\
\hline & MR1371_OT1_R & CTTTTGACTTTTTAGGTTGACTTCCAGCCATG \\
\hline & MR1372_OT2_F & CCGCATACATATTACCCACAATTCCСTTTCC \\
\hline & MR1373_OT2_R & CATTCTGCCGTTTGTTACTTACCAATGCC \\
\hline & MR1374_OT3_F & GAGACAGAGTCTTACTTTGTCACCCAGGCTG \\
\hline & MR1375_OT3_R & GAATGAAGAAGGAAAAGTCTCTCATTCATTGCATC \\
\hline
\end{tabular}

Table 12: Overview of parameters used for RESOLFT imaging of endogenously tagged cells.

\begin{tabular}{llll}
\hline Cell line & VIM-rsEGFP2 & ZYX-rsEGFP2 & HMG-I-rsEGFP2 \\
\hline \multirow{2}{*}{ Pixel size (nm) } & 20 (overview) & & 30 (x-axis) \\
& $\begin{array}{l}15 \text { (inset) } \\
30 \text { (movie) }\end{array}$ & 30 & 25 (y-axis) \\
\hline Overall dwell time ( $\mu \mathrm{s})$ & 520 & 1000 & 780 \\
\hline ON switching at 405nm & $50 \mu \mathrm{s}$ & $730 \mu \mathrm{s}$ & $50 \mu \mathrm{s}$ \\
(Gaussian shaped) & $0.8 \mu \mathrm{W}$ & $2.6 \mu \mathrm{W}$ & $0.8 \mu \mathrm{W}$ \\
Time/Average Powerl Intensity & $2 \mathrm{~kW} / \mathrm{cm}^{2}$ & $2 \mathrm{~kW} / \mathrm{cm}^{2}$ & $2 \mathrm{~kW} / \mathrm{cm}^{2}$ \\
\hline OFF switching at 491 nm & $400 \mu \mathrm{s}$ & $730 \mu \mathrm{s}$ & $650 \mu \mathrm{s}$ \\
(donut shaped) & $8.8 \mu \mathrm{W}$ & $2.6 \mu \mathrm{W}$ & $3 \mu \mathrm{W}$ \\
Time/Average Powerl Intensity & $6.5 \mathrm{~kW} / \mathrm{cm}^{2}$ & $2 \mathrm{~kW} / \mathrm{cm}^{2}$ & $2.2 \mathrm{~kW} / \mathrm{cm}^{2}$ \\
\hline
\end{tabular}




\begin{tabular}{llll}
\hline Read out at $491 \mathrm{~nm}$ & $50 \mu \mathrm{s}$ & $200 \mu \mathrm{s}$ & $50 \mu \mathrm{s}$ \\
(Gaussian shaped) & $2.4 \mu \mathrm{W}$ & $4.8 \mu \mathrm{W}$ & $4 \mu \mathrm{W}$ \\
TimelAverage Powerl Intensity & $5.3 \mathrm{~kW} / \mathrm{cm}^{2}$ & $2.6 \mathrm{~kW} / \mathrm{cm}^{2}$ & $2.2 \mathrm{~kW} / \mathrm{cm}^{2}$ \\
\hline
\end{tabular}

Table 13: Overview of all generated gRNA/Cas9 plasmids (pMR) during this study.

\begin{tabular}{|c|c|c|c|c|}
\hline pMR & Gene & Target site (5' to 3 ') & PAM & Score \\
\hline 1 & \multirow{3}{*}{ ACTB } & GCCGCGCTCGTCGTCGACAA & CGG & 89 \\
\hline 2 & & TTCCGGCGCGCCGAGTCCTT & AGG & 92 \\
\hline 3 & & ACCTCGGCTCACAGCGCGCC & CGG & 88 \\
\hline 4 & \multirow{4}{*}{ CEP41 } & GTCACCTGCAAGGCAAACCC & TGG & 64 \\
\hline 5 & & CGAAGCGCTCAGAATCTGCC & AGG & 77 \\
\hline 6 & & CTGGCAGATTCTGAGCGCTT & CGG & 74 \\
\hline 7 & & ACCCAACTTGGGAAATGCTC & AGG & 57 \\
\hline 8 & \multirow{2}{*}{$\mathrm{CHCHD} 3$} & GAAAGAGTAAGTTTTACCAC & CGG & 51 \\
\hline 9 & & CTTGCAGAGCATGCTTGAGA & AGG & 64 \\
\hline 10 & \multirow{2}{*}{ DNM1L } & GATTTCAGCAATAATTTGAC & TGG & 63 \\
\hline 11 & & CСTTGTAATGCCTGCATTAA & AGG & 51 \\
\hline 12 & \multirow{3}{*}{ EGFR } & TCCTGGGTATCGAAAGAGTC & TGG & 80 \\
\hline 13 & & TCCAGACTCTTTCGATACCC & AGG & 93 \\
\hline 14 & & GACCTGCTGTGGCTTGGTCC & TGG & 72 \\
\hline 15 & GJA4 & TGTTGGGTGACATAAGCCAC & AGG & 61 \\
\hline 16 & \multirow{2}{*}{ HIST1H2BJ } & CGCCGTGTCCGAGGGTACTA & AGG & 68 \\
\hline 17 & & GGCCTTAGTACCCTCGGACA & CGG & 39 \\
\hline 18 & \multirow{2}{*}{ IMMT } & TATGCCAGCGCCGTAGGAAT & AGG & 81 \\
\hline 19 & & CTGAGTGGTTCCTATTCCTA & CGG & 60 \\
\hline 20 & \multirow{3}{*}{ MINOS1 } & TGTCCTTGCTTTACAGGAGC & AGG & 54 \\
\hline 21 & & ACCTGATACAGCAATTAGAG & AGG & 71 \\
\hline 22 & & СССТСTCTAATTGCTGTATC & AGG & 75 \\
\hline 23 & \multirow{2}{*}{ NUP98 } & AAATCATTTGGAACACCCTT & TGG & 59 \\
\hline 24 & & ACACCCTTTGGGGGTGGCAC & AGG & 59 \\
\hline 25 & \multirow{2}{*}{ TOMM70A } & ACAGAAGTTGCAAAGAAATA & CGG & 20 \\
\hline 26 & & GGCCATCTCCATTTCCGATT & TGG & 77 \\
\hline 27 & \multirow{5}{*}{ VIM } & ATAATTTAGTCTTTGGCATG & TGG & 36 \\
\hline 28 & & ATATAGGATAATTTAGTCTT & TGG & 44 \\
\hline 29 & & GCGCAAGATAGATTTGGAAT & AGG & 68 \\
\hline 30 & & TCAGGAGCGCAAGATAGATT & TGG & 65 \\
\hline 31 & & TTTTATTTGCCGTGATATAT & AGG & 53 \\
\hline 32 & \multirow{2}{*}{ RAB4A } & CGTACCGTAGGTTTCGGACA & TGG & 97 \\
\hline 33 & & GTCCGCCGGCGCGTCTCGGT & GGG & 92 \\
\hline 34 & \multirow{2}{*}{ MAP1LC3A } & GCGGGGATGTGTCTGCGGTT & TGG & 83 \\
\hline 35 & & GGCTCCGCGCCTCGCGCTCA & AGG & 83 \\
\hline
\end{tabular}




\begin{tabular}{|c|c|c|c|c|}
\hline 36 & \multirow{2}{*}{ ZYX } & GACAGAGCACGTGACCGTCC & AGG & 84 \\
\hline 37 & & TGTCATCTGCCTCAATCGAC & AGG & 87 \\
\hline 38 & \multirow{2}{*}{ MAPRE3 } & GCCGCCCTCAGTACTCGTCC & TGG & 87 \\
\hline 39 & & CAGGGGGTGCGAATCCTTCC & TGG & 78 \\
\hline 40 & \multirow{2}{*}{ PFN1 } & GTCGATGTAGGCGTTCCACC & CGG & 93 \\
\hline 41 & & GTAGGCGTTCCACCCGGCCA & TGG & 88 \\
\hline 42 & \multirow{2}{*}{ B4GALT1 } & CGTGTACCAAAACGCTAGCT & CGG & 95 \\
\hline 43 & & AGAGGTCCCTGGCTAATTTC & AGG & 65 \\
\hline 44 & \multirow{2}{*}{ TGOLN2 } & ACGAGAGCAGCACAATCCAT & TGG & 66 \\
\hline 45 & & ATACCATTCTGTTAGGACTT & AGG & 62 \\
\hline 46 & \multirow{2}{*}{ BID } & TGACTGGCTAAAGCTCGATG & TGG & 88 \\
\hline 47 & & TTCCAGTGTAGACGGAGCCC & TGG & 74 \\
\hline 48 & \multirow{2}{*}{ PDHA1 } & AACTTCAAAAGGTGGGTCGC & TGG & 84 \\
\hline 49 & & CACCACGAACTTCAAAAGGT & GGG & 66 \\
\hline 50 & \multirow{2}{*}{ CALR } & GCCTCTCTACAGCTCGTCCT & TGG & 81 \\
\hline 51 & & TCTACAGCTCGTCCTTGGCC & TGG & 63 \\
\hline 52 & \multirow{2}{*}{ ATP5A1 } & АTCTTTCCATCAGCССТАTT & TGG & 74 \\
\hline 53 & & TTATTTCCAAATAGGGCTGA & TGG & 57 \\
\hline 54 & \multirow{2}{*}{ PXMP2 } & CGAGGCGCCGAGAGCGGAGT & GGG & 80 \\
\hline 55 & & TCCCCAGCGCCGTGCCGCCG & GGG & 75 \\
\hline 56 & \multirow{2}{*}{ CKAP4 } & GCGCGGCCCGGGAAACTTGC & AGG & 87 \\
\hline 57 & & CGCGGCCCGGGAAACTTGCA & GGG & 82 \\
\hline 58 & \multirow{2}{*}{ HIST1H1A } & AAGAGCCACCTACGCATTTC & AGG & 81 \\
\hline 59 & & CTTTTCCTGAAATGCGTAGG & TGG & 80 \\
\hline 60 & \multirow{2}{*}{ HMGA1 } & GAGGAGCAGGCGGCACGCAT & GGG & 70 \\
\hline 61 & & CCAACAACTGCCСАССТСАС & AGG & 51 \\
\hline 62 & \multirow{2}{*}{ TGFBR2 } & GTGTTTAGGGAGCCGTCTTC & AGG & 84 \\
\hline 63 & & AGCATCTGGACAGGCTCTCG & GGG & 68 \\
\hline 64 & \multirow{2}{*}{ RAB1B } & TGGTGGCCGCTCCGCCCCAG & AGG & 62 \\
\hline 65 & & TTCGGGGTTCATGGCGGCGG & CGG & 44 \\
\hline 66 & \multirow{2}{*}{ SAM50 } & AACCGCGTTTCCTCGAGAGA & CGG & 93 \\
\hline 67 & & CCTGTAGGGGTGTCGGCTAC & AGG & 91 \\
\hline 68 & \multirow{2}{*}{ FIS1 } & GAACCACTTCCGGCGTCCGG & CGG & 93 \\
\hline 69 & & ACTGTAGTGTGAGGCTCGCG & GGG & 88 \\
\hline 70 & \multirow{2}{*}{ TIMM50 } & TGGACTTGGAGAGCTCGGCC & AGG & 67 \\
\hline 71 & & GAGGTTCTGCTTGTTGGACT & TGG & 61 \\
\hline 72 & \multirow{4}{*}{ OPA1 } & TTTCATTGAAGCTCTTCATC & AGG & 55 \\
\hline 73 & & TAAAAATTCTCTAACTGTAT & TGG & 49 \\
\hline 74 & & TTACAGCATAAGTGACAAGC & AGG & 53 \\
\hline 75 & & TTTCAAAGGTATCACAAATC & AGG & 44 \\
\hline 76 & MFN1 & AGTTTCGTTTCCTCCTATCA & TGG & 62 \\
\hline
\end{tabular}




\begin{tabular}{|c|c|c|c|c|}
\hline 77 & & GCTTTGGTGACCATGATAGG & AGG & 49 \\
\hline 78 & & CCAAAGCAATCTCTATTGTT & AGG & 49 \\
\hline 79 & & GACCTTTATAGTAATTAGAT & TGG & 58 \\
\hline 80 & \multirow{3}{*}{ MFN2 } & CAGGAATAAAGCCGGTTGGT & TGG & 82 \\
\hline 81 & & GCCCACATGGCACTTAGGGC & TGG & 75 \\
\hline 82 & & TTTGCAGGAATAAAGCCGGT & TGG & 73 \\
\hline 83 & \multirow{2}{*}{ RAB5A } & CGAGGCGCAACAAGACCCAA & CGG & 82 \\
\hline 84 & & CTTCTGGGAGAGTCCGCTGT & TGG & 78 \\
\hline 85 & \multirow{2}{*}{ COX8A } & ACAGGATCCAGCCCGCTGGC & AGG & 74 \\
\hline 86 & & CATGACCAGGATGGGCCGGT & GGG & 73 \\
\hline 87 & \multirow{2}{*}{ RAB7A } & GGTCATCCTTCAAACTAAAG & GGG & 63 \\
\hline 88 & & GAGGTCATCCTTCAAACTAA & AGG & 60 \\
\hline 89 & \multirow{2}{*}{ RAB9A } & CTTTTGAGATTAACAATGGC & AGG & 56 \\
\hline 90 & & AGAAGAATGTGTAGGTATAA & AGG & 42 \\
\hline 91 & \multirow{2}{*}{ RAB11A } & GAGCGCCGAGCTTCAACTGC & CGG & 89 \\
\hline 92 & & GACCAGGGGGCGTCGCTGCA & GGG & 72 \\
\hline 93 & \multirow{2}{*}{ BCL2 } & GGAGAACAGGGTACGATAAC & CGG & 87 \\
\hline 94 & & GTCGCAGAGGGGCTACGAGT & GGG & 87 \\
\hline 95 & \multirow{2}{*}{ BAX } & GCCTCTCGCCGGGTCCGCGC & GGG & 82 \\
\hline 96 & & TCTCGCCGGGTCCGCGCGGG & CGG & 80 \\
\hline 97 & \multirow{2}{*}{ VDAC1 } & AGTGTCAGTTTAATACCTGC & AGG & 68 \\
\hline 98 & & GTGTCAGTTTAATACCTGCA & GGG & 66 \\
\hline 99 & \multirow{2}{*}{ CLTB } & CATTTTCCCCGCGCCTCCGC & CGG & 84 \\
\hline 100 & & GACGCTGTCACCCGAGCCGC & GGG & 81 \\
\hline 101 & \multirow{2}{*}{ RAB15 } & TCGTACTGCTTCGCCATGAC & TGG & 91 \\
\hline 102 & & TGTCGGTGAAGCGGCACAGC & AGG & 74 \\
\hline 103 & \multirow{2}{*}{ CHCHD6 } & CAGGGCGATCTGCACGGAGC & TGG & 78 \\
\hline 104 & & TGGGAAGCGGTTGCTGTCAC & AGG & 69 \\
\hline 105 & \multirow{2}{*}{ MTX2 } & CAGCACTATTTTGAAGATCG & TGG & 66 \\
\hline 106 & & TTGAAGATCGTGGTAAAGGC & AGG & 65 \\
\hline 107 & \multirow{2}{*}{ HSPA9 } & CAGATGGCATCTGAGCGAGA & AGG & 70 \\
\hline 108 & & GCATCTGAGCGAGAAGGCTC & TGG & 50 \\
\hline 109 & \multirow{2}{*}{ APOOL } & GCCCGTGATCCATGAGCTTG & GGG & 71 \\
\hline 110 & & CTGCCCGTGATCCATGAGCT & TGG & 67 \\
\hline 111 & \multirow{2}{*}{ PARP-1 } & GGTAGCCGAGTCACACCCGG & TGG & 87 \\
\hline 112 & & CCATCAGCAACTTAGCGGCC & AGG & 85 \\
\hline 113 & \multirow{2}{*}{ TFAM } & CTTAATTTTACGATAGCTTC & AGG & 64 \\
\hline 114 & & TTCAGGTATTGAGACCTAAC & TGG & 55 \\
\hline 115 & \multirow{2}{*}{ ALEX3 } & CCACCTTGTTTATATGGTAA & AGG & 55 \\
\hline 116 & & GAACATATGTTCAGCAAGTT & TGG & 54 \\
\hline 117 & RHOT1 & ACCTCAAGAGCTCCACGTTT & TGG & 70 \\
\hline
\end{tabular}




\begin{tabular}{|c|c|c|c|c|}
\hline 118 & & GCCAAAACGTGGAGCTCTTG & AGG & 67 \\
\hline 119 & \multirow{2}{*}{ RHOT2 } & ACCTGGGTGTGCCTCGCTGC & TGG & 72 \\
\hline 120 & & GGGGGTTGTCGGGGCCGCCG & TGG & 65 \\
\hline 121 & \multirow{2}{*}{ POM121 } & GGTCCAAGGCTCTTCCTAGC & AGG & 66 \\
\hline 122 & & GCTCTTCCTAGCAGGTGCCA & AGG & 50 \\
\hline 123 & \multirow{2}{*}{ NUP93 } & ATGATACCATACCGAACGTC & TGG & 95 \\
\hline 124 & & CGGCACACTGTCAGTACATC & AGG & 82 \\
\hline 125 & \multirow{2}{*}{ NUP107 } & GACCAGGGACTTGACCCATT & AGG & 79 \\
\hline 126 & & TCTCTAATGCTCCTAGACCA & GGG & 72 \\
\hline 127 & \multirow{2}{*}{ PHB1 } & TGCACCTCCGCGGGCTGACT & GGG & 55 \\
\hline 128 & & CTGCACCTCCGCGGGCTGAC & TGG & 55 \\
\hline 129 & \multirow{2}{*}{ PHB2 } & GGGTGGAGTTCTTGGTGACT & AGG & 56 \\
\hline 130 & & CTGGCTGGCTCCTCAAAAAC & TGG & 69 \\
\hline 131 & \multirow{2}{*}{ RTN1 } & GTTTGTGTCCAGTCCCCGGT & GGG & 83 \\
\hline 132 & & TCAGCATGCCTCTTAGCGCC & TGG & 85 \\
\hline 133 & \multirow{2}{*}{ RTN4 } & TTCATTTGATTATACGGGGG & AGG & 84 \\
\hline 134 & & TGATTATACGGGGGAGGGTC & AGG & 80 \\
\hline 135 & \multirow{2}{*}{ CCND1 } & TGGCATCGGGGTACGCGCGG & CGG & 95 \\
\hline 136 & & GTCCTGGCTGGGTCCGCGCT & CGG & 71 \\
\hline 137 & \multirow{2}{*}{ CCNE1 } & CTGCGCTCCCTCGGCATGAT & GGG & 87 \\
\hline 138 & & CCTGCGCTCCCTCGGCATGA & CGG & 86 \\
\hline 139 & \multirow{2}{*}{ CCNA2 } & TGCACGCTGCTTGGCGCCGC & AGG & 89 \\
\hline 140 & & CGACTATTCTTTGGCCGGGT & CGG & 88 \\
\hline 141 & \multirow{2}{*}{ CCNB1 } & CTTCGGACTGCGAACTAACG & CGG & 97 \\
\hline 142 & & CGTTAGTTCGCAGTCCGAAG & CGG & 97 \\
\hline 143 & \multirow{2}{*}{ OXA1 } & CAGCCAAGTGTGTCGTGCCA & GGG & 84 \\
\hline 144 & & CTTATGTTCTGTGCGCATTC & TGG & 81 \\
\hline 145 & \multirow{2}{*}{ ITPR1 } & TACGCGGAGGGATCGACAAA & TGG & 96 \\
\hline 146 & & ATTTGTTCTCTGTACGCGGA & GGG & 92 \\
\hline 147 & \multirow{2}{*}{ MFF } & GCAGTGACACATCACTAGGA & AGG & 63 \\
\hline 148 & & TTCAGTTGAGCTGGTATTAT & AGG & 52 \\
\hline 149 & \multirow{2}{*}{ PARK2 } & CGGGGCGTGGCGCCATACCG & GGG & 95 \\
\hline 150 & & GAACAGGCCCATGCGCGCAG & CGG & 89 \\
\hline 151 & \multirow{2}{*}{ PINK1 } & TGGCATCCTCTGTGTCGTGA & TGG & 73 \\
\hline 152 & & GGAGTGTGAAACGCTCTGCC & AGG & 71 \\
\hline 153 & \multirow{2}{*}{ GDAP1 } & AATGAGCTTAACCTCCGCGT & CGG & 94 \\
\hline 154 & & AAACCCGGAAACGCCTTGCG & GGG & 92 \\
\hline 155 & \multirow{2}{*}{ TOMM70A } & ССССTGTTTTATAATGTTGG & TGG & 71 \\
\hline 156 & & ATCGCAAAGTGAATACAGAT & GGG & 69 \\
\hline 157 & \multirow{2}{*}{$\mathrm{CHCHD} 3$} & GTAAGTTTTACCACCGGGAG & AGG & 90 \\
\hline 158 & & TATTTTTTGTCCTCTCCCGG & TGG & 74 \\
\hline
\end{tabular}




\begin{tabular}{|c|c|c|c|c|}
\hline 159 & \multirow{2}{*}{ MINOS1 } & CTGTATCAGGTCAGACCCAA & AGG & 73 \\
\hline 160 & & GCTGAATGTCCTTGCTTTAC & AGG & 72 \\
\hline 161 & \multirow{2}{*}{ IMMT } & GGTTCCTATTCCTACGGCGC & TGG & 95 \\
\hline 162 & & AGGGTTCGCAGCGAGAGTCC & CGG & 87 \\
\hline 163 & HSPA9 & AGCTTCATATGTTGTCCTTC & TGG & 52 \\
\hline 164 & \multirow{2}{*}{ ATG5 } & GAGTGAACATCTGAGCTACC & CGG & 79 \\
\hline 165 & & GTCACCCTTTTGCTTCAATC & AGG & 77 \\
\hline 166 & \multirow{2}{*}{ NUP93 } & CATTGGTGTCCCCAGACGTT & CGG & 85 \\
\hline 167 & & GTGTCCCCAGACGTTCGGTA & TGG & 94 \\
\hline 168 & \multirow{2}{*}{ NUP107-N } & ACCTATTTCGTTTCGCCAAC & AGG & 95 \\
\hline 169 & & GTTTCGCCAACAGGCGAGAC & GGG & 94 \\
\hline 170 & \multirow{2}{*}{ CAV1 } & ACAGACGGTGTGGACGTAGA & TGG & 90 \\
\hline 171 & & ACCСАСТСTTTGAAGCTGTT & GGG & 74 \\
\hline 172 & \multirow{2}{*}{ TUBA1B } & TCGAGGTAAGTAACGCACTA & GGG & 96 \\
\hline 173 & & AGCCCTTCCCGGCTGTATAC & AGG & 88 \\
\hline 174 & \multirow{2}{*}{ VDAC2 } & САCTCTCTGCTCTGGTAGAT & GGG & 50 \\
\hline 175 & & GATGGGAAGAGCATTAATGC & TGG & 42 \\
\hline 176 & \multirow{2}{*}{ NUP133 } & CAAGCTGATCCGCTTGTAGC & AGG & 94 \\
\hline 177 & & TTCAAAACAAACTCGAAGTA & AGG & 73 \\
\hline 178 & \multirow{2}{*}{ TRAK1 } & AAACAAACTAGCTTACGGTG & AGG & 90 \\
\hline 179 & & AACTAGCTTACGGTGAGGAC & TGG & 89 \\
\hline 180 & \multirow{2}{*}{ TRAK2 } & GCAAAGCTACCCATTATGAC & TGG & 81 \\
\hline 181 & & TTGGGTGAGGATGTGCAAAC & TGG & 73 \\
\hline 182 & \multirow{2}{*}{ gP210 } & GAACCTTCACGCGGCCTAGT & GGG & 95 \\
\hline 183 & & TTCCATCTTGGGGGTGCACG & AGG & 80 \\
\hline 184 & \multirow{3}{*}{ EGFR } & AACGTTTACACCGACTAGCC & AGG & 91 \\
\hline 185 & & TCTAAGAGCTAATGCGGGCA & TGG & 88 \\
\hline 186 & & GTGGGTCTAAGAGCTAATGC & GGG & 82 \\
\hline 187 & \multirow{3}{*}{$\mathrm{H} 2 \mathrm{BJ}$} & GCGCTAAGTAAACAGTGAGT & TGG & 67 \\
\hline 188 & & GGCTCTTAAAAGAGCCGTTA & GGG & 76 \\
\hline 189 & & TTGCAAАСТСТСААСССТАA & CGG & 71 \\
\hline 190 & \multirow{6}{*}{ DLK1_Mm } & CATGTGGTTGTAGCGCAGGT & TGG & 91 \\
\hline 191 & & GTAGCGCAGGTTGGACACCC & AGG & 84 \\
\hline 192 & & GAAGCATGTGGTTGTAGCGC & AGG & 87 \\
\hline 193 & & CATGTGGTTGTAGCGCAGGT & TGG & 91 \\
\hline 194 & & GTAGCGCAGGTTGGACACCC & AGG & 84 \\
\hline 195 & & GAAGCATGTGGTTGTAGCGC & AGG & 87 \\
\hline 196 & \multirow{4}{*}{ PHB1 } & AGCCCGCGGAGGTGCAGGCA & GGG & 24 \\
\hline 197 & & GCTGTGGCCCAGTCAGCCCG & CGG & 39 \\
\hline 198 & & CAGTCAGCCCGCGGAGGTGC & AGG & 41 \\
\hline 199 & & GGCTGTGTTAAGAATCATCG & GGG & 23 \\
\hline
\end{tabular}




\begin{tabular}{|c|c|c|c|c|}
\hline 200 & & AAGGCTGTGTTAAGAATCAT & CGG & 20 \\
\hline 201 & \multirow{8}{*}{ PHB2 } & TACAGTGACAGCCTCATCAA & GGG & 64 \\
\hline 202 & & TGTGAGAGGCAGGACACTCT & AGG & 60 \\
\hline 203 & & СТCАTTTCTTACССTTGATG & AGG & 62 \\
\hline 204 & & ACCAAGAACTCCACCCCCAG & AGG & 45 \\
\hline 205 & & CAGGGTAGGAGGGTCGTAGA & GGG & 78 \\
\hline 206 & & TGTGAGAGGCAGGACACTCT & AGG & 60 \\
\hline 207 & & TACAGTGACAGCCTCATCAA & GGG & 64 \\
\hline 208 & & TTAGGGGACATAAATGTGAG & AGG & 57 \\
\hline 209 & \multirow{3}{*}{ STOML2 } & ATCGAGTCAAGATGAGTTAG & TGG & 78 \\
\hline 210 & & CAAGACTTGCATCTGTACCC & TGG & 76 \\
\hline 211 & & TCAAGATGAGTTAGTGGAGC & TGG & 70 \\
\hline 212 & \multirow{3}{*}{ PHB1_Exon2 } & GACTCATTTTCTCATCCCGT & GGG & 69 \\
\hline 213 & & GGACTCATTTTCTCATCCCG & TGG & 61 \\
\hline 214 & & TGACCGATTCCGTGGAGTGC & AGG & 47 \\
\hline 215 & \multirow{3}{*}{ PHB2_Exon1 } & TTCGCGCACACCGTAGGCCA & CGG & 94 \\
\hline 216 & & CCGCCAAGTCCTTCAAGTTC & TGG & 72 \\
\hline 217 & & TTGGCGGGACGGCTGCCCGC & CGG & 82 \\
\hline 218 & \multirow{3}{*}{ PHB2_Exon2 } & ATCTTCTTCAATCGGATCGG & TGG & 97 \\
\hline 219 & & TCGGATCGGTGGAGTGCAGC & AGG & 86 \\
\hline 220 & & CAGGACACTATCCTGGCCGA & GGG & 82 \\
\hline 221 & \multirow{2}{*}{ PHB1 } & TGCACCTCCGCGGGCTGACT & GGG & 53 \\
\hline 222 & & CTGCACCTCCGCGGGCTGAC & TGG & 53 \\
\hline 223 & \multirow{2}{*}{ PHB2 } & GGGTGGAGTTCTTGGTGACT & AGG & 56 \\
\hline 224 & & CTGGCTGGCTCCTCAAAAAC & TGG & 55 \\
\hline 225 & \multirow{3}{*}{ PHB1_Exon2 } & GACTCATTTTCTCATCCCGT & GGG & 69 \\
\hline 226 & & GGACTCATTTTCTCATCCCG & TGG & 61 \\
\hline 227 & & TGACCGATTCCGTGGAGTGC & AGG & 47 \\
\hline 228 & \multirow{3}{*}{ PHB2_Exon1 } & TTCGCGCACACCGTAGGCCA & CGG & 94 \\
\hline 229 & & CCGCCAAGTCCTTCAAGTTC & TGG & 72 \\
\hline 230 & & TTGGCGGGACGGCTGCCCGC & CGG & 82 \\
\hline 231 & \multirow{3}{*}{ PHB2_Exon2 } & ATCTTCTTCAATCGGATCGG & TGG & 97 \\
\hline 232 & & TCGGATCGGTGGAGTGCAGC & AGG & 86 \\
\hline 233 & & CAGGACACTATCCTGGCCGA & GGG & 82 \\
\hline 234 & \multirow{3}{*}{ PHB1-N } & GCTGGACCCTCTCACACCTA & AGG & 78 \\
\hline 235 & & ACAACAGTCACTTTTCAACC & TGG & 71 \\
\hline 236 & & TTATCCCCTTAGGTGTGAGA & GGG & 71 \\
\hline 237 & MIC60_Exon1 & GCTGCGGGCCTGTCAGTTAT & CGG & 84 \\
\hline 238 & \multirow{2}{*}{ MIC60_Exon2 } & TATCTGCGGCATGGTCGCAA & TGG & 94 \\
\hline 239 & & GGCATGGTCGCAATGGACGG & AGG & 92 \\
\hline 240 & MIC10_Exon1 & TGTCTGAGTCGGAGCTCGGC & AGG & 88 \\
\hline
\end{tabular}




\begin{tabular}{|c|c|c|c|c|}
\hline 241 & & CGACCGCATCCGCCAGACAC & CGG & 86 \\
\hline 242 & & CAGGAAGTGGGACCGGTGTC & TGG & 78 \\
\hline 252 & \multirow{5}{*}{ ATP5B } & GCTGAAGAGCATTCATCGTG & AGG & 85 \\
\hline 253 & & TGAAGAGCATTCATCGTGAG & GGG & 83 \\
\hline 254 & & CTGAAGAGCATTCATCGTGA & GGG & 81 \\
\hline 255 & & AGCTTTTTGGGTTAGGGGCA & AGG & 55 \\
\hline 256 & & AAATGAAGCTTTTTGGGTTA & GGG & 50 \\
\hline 257 & \multirow{6}{*}{ CLS1 } & ATGTTGTCAATGACGAGAAT & TGG & 82 \\
\hline 258 & & GTCAATGACGAGAATTGGCT & TGG & 79 \\
\hline 259 & & AATTGGCTTGGCCCCAGTTC & TGG & 72 \\
\hline 260 & & ATGGATTTATTGCTCGAAAC & TGG & 90 \\
\hline 261 & & TGGATTTATTGCTCGAAACT & GGG & 86 \\
\hline 262 & & GGCCAATCAAAGATCAGCTT & TGG & 71 \\
\hline
\end{tabular}

Table 14: Overview of all generated donor plasmids (pDM) within this study.

\begin{tabular}{rlllll}
\hline pDM & Gene & Terminus & Transgene & gRNA site & Cloning strategy \\
\hline 1 & ACTB & N & Dreiklang & present & Gene synthesis \\
\hline 4 & ATP5A1 & C & Dreiklang & absent & Gibson Assembly \\
\hline 5 & B4GALT1 & C & Dreiklang & present & Gibson Assembly \\
\hline 7 & BCL2 & N & Dreiklang & absent & Gibson Assembly \\
\hline 10 & CCNA2 & N & Dreiklang & present & Gibson Assembly \\
\hline 11 & CCNB1 & N & Dreiklang & present & Gibson Assembly \\
\hline 15 & CHCHD3 & C & Dreiklang & present & 4-Fragment cloning \\
\hline 16 & CHCHD6 & C & Dreiklang & absent & Gibson Assembly \\
\hline 19 & COX8A & C & Dreiklang & absent & Gibson Assembly \\
\hline 20 & DNM1L & C & Dreiklang & absent & 4-Fragment cloning \\
\hline 22 & FIS1 & N & Dreiklang & present & Gibson Assembly \\
\hline 23 & GDAP1 & N & Dreiklang & absent & Gibson Assembly \\
\hline 24 & H1A & C & Dreiklang & present & Gibson Assembly \\
\hline 25 & H2BJ & C & Dreiklang & present & 4-Fragment cloning \\
\hline 26 & HMGA1 & C & Dreiklang & present & Gibson Assembly \\
\hline 27 & HSPA9 & C & Dreiklang & absent & Gibson Assembly \\
\hline 28 & IMMT & C & Dreiklang & absent & 4-Fragment cloning \\
\hline 29 & ITPR1 & N & Dreiklang & absent & Gibson Assembly \\
\hline 30 & MAP1LC3A & N & Dreiklang & present & Gibson Assembly \\
\hline 31 & MAPRE3 & C & Dreiklang & present & Gibson Assembly \\
\hline 32 & MFF & N & Dreiklang & absent & Gibson Assembly \\
\hline 33 & MFN1 & C & Dreiklang & present & Gibson Assembly \\
\hline 34 & MFN2 & C & Dreiklang & absent & Gibson Assembly \\
\hline 35 & MINOS1 & C & Dreiklang & absent & Gibson Assembly \\
\hline 36 & MTX2 & C & Dreiklang & absent & Gibson Assembly \\
\hline & & & & & \\
\hline
\end{tabular}




\begin{tabular}{|c|c|c|c|c|c|}
\hline 39 & NUP107-N & $\mathrm{N}$ & Dreiklang & present & Gibson Assembly \\
\hline 40 & OPA1 & C & Dreiklang & absent & Gibson Assembly \\
\hline 43 & PARP1 & C & Dreiklang & present & Gibson Assembly \\
\hline 45 & PFN1 & $\mathrm{N}$ & Dreiklang & present & Gibson Assembly \\
\hline 46 & PHB1 & C & Dreiklang & present & Gibson Assembly \\
\hline 47 & PHB2 & C & Dreiklang & present & Gibson Assembly \\
\hline 52 & RAB4 & $\mathrm{N}$ & Dreiklang & present & Gibson Assembly \\
\hline 53 & RAB5A & $\mathrm{N}$ & Dreiklang & absent & Gibson Assembly \\
\hline 54 & RAB7A & $\mathrm{N}$ & Dreiklang & present & Gibson Assembly \\
\hline 55 & RAB9A & $\mathrm{N}$ & Dreiklang & present & Gibson Assembly \\
\hline 58 & RHOT1 & C & Dreiklang & absent & Gibson Assembly \\
\hline 59 & RHOT2 & C & Dreiklang & absent & Gibson Assembly \\
\hline 61 & RTN4 & C & Dreiklang & present & Gibson Assembly \\
\hline 62 & SAMM50 & C & Dreiklang & present & Gibson Assembly \\
\hline 63 & TFAM & C & Dreiklang & present & Gibson Assembly \\
\hline 65 & TGOLN2 & C & Dreiklang & present & Gibson Assembly \\
\hline 67 & TOMM70A & C & Dreiklang & absent & 4-Fragment cloning \\
\hline 69 & VDAC & C & Dreiklang & absent & Gibson Assembly \\
\hline 70 & VIM & C & Dreiklang & present & 4-Fragment cloning \\
\hline 71 & VIM & C & rsEGFP2 & present & 4-Fragment cloning \\
\hline 72 & ZYX & C & Dreiklang & absent & Gibson Assembly \\
\hline 73 & ACTB & C & rsEGFP2 & present & pDM1 + rsEGFP2 \\
\hline 74 & ATG5 & C & Dreiklang & present & Gibson Assembly \\
\hline 76 & NUP133 & C & Dreiklang & present & Gibson Assembly \\
\hline 77 & gP210 & C & Dreiklang & present & Gibson Assembly \\
\hline 78 & VDAC2 & C & Dreiklang & present & Gibson Assembly \\
\hline 81 & $\mathrm{H} 2 \mathrm{BJ}$ & C & rsEGFP2 & present & pDM25 + rsEGFP2 \\
\hline 82 & $\mathrm{H} 1 \mathrm{~A}$ & C & rsEGFP2 & present & pDM24 + rsEGFP2 \\
\hline 83 & HMGA1 & C & rsEGFP2 & present & pDM26 + rsEGFP2 \\
\hline 84 & PHB1 & C & rsEGFP2 & present & pDM46 + rsEGFP2 \\
\hline 85 & PHB2 & C & rsEGFP2 & present & pDM47 + rsEGFP2 \\
\hline 86 & RAB7A & C & rsEGFP2 & present & pDM54 + rsEGFP2 \\
\hline 87 & RTN4 & C & rsEGFP2 & present & pDM61 + rsEGFP2 \\
\hline 89 & $Z Y X$ & C & rsEGFP2 & absent & pDM72 + rsEGFP2 \\
\hline 90 & $\mathrm{H} 1 \mathrm{~A}$ & $\mathrm{C}$ & Halo & present & pDM24 + Halo \\
\hline 91 & HMGA1 & C & Halo & present & pDM26 + Halo \\
\hline 92 & PHB1 & C & Halo & present & pDM46 + Halo \\
\hline 93 & PHB2 & C & Halo & present & pDM47 + Halo \\
\hline 94 & RAB7A & C & Halo & present & pDM54 + Halo \\
\hline 95 & ZYX & C & Halo & absent & pDM72 + Halo \\
\hline 98 & $\mathrm{H} 2 \mathrm{BJ}$ & $\mathrm{C}$ & Halo & present & pDM25 + Halo \\
\hline
\end{tabular}




\begin{tabular}{|c|c|c|c|c|c|}
\hline 99 & VIM & C & Halo & present & pDM70 + Halo \\
\hline 100 & EGFR & C & Dreiklang & present & Gibson Assembly \\
\hline 101 & VIM & C & rsEGFP2 & absent & SDM on pDM71, no gRNA1/2 sites \\
\hline 102 & HMGA1 & C & rsEGFP2 & absent & SDM on pDM83, no gRNA1 site \\
\hline 103 & HMGA1 & C & rsEGFP2 & absent & SDM on pDM83, no gRNA2 site \\
\hline 104 & PHB1 & C & Dreiklang & absent & SDM on pDM46, no gRNA1/2 sites \\
\hline 105 & PHB1 & C & Halo & absent & SDM on pDM92, no gRNA1/2 sites \\
\hline 106 & PHB2 & C & Dreiklang & absent & SDM on pDM47, no gRNA2 site \\
\hline 107 & PHB2 & C & rsEGFP2 & absent & SDM on pDM85, no gRNA2 site \\
\hline 108 & PHB2 & C & Halo & absent & SDM on pDM93, no gRNA2 site \\
\hline 109 & SLP2 & C & DK & absent & Gibson Assembly \\
\hline 110 & PHB1 & C & 1X-FLAG & absent & oligo cloning into pDM104 \\
\hline 111 & PHB2 & C & $1 x-F L A G$ & absent & oligo cloning into pDM106 \\
\hline 112 & PHB & $\mathrm{N}$ & Dreiklang & present & Gibson Assembly \\
\hline 113 & PHB1 & C & EGFP & absent & pDM104 + EGFP \\
\hline 114 & PHB2 & C & EGFP & absent & pDM106 + EGFP \\
\hline 115 & PHB1 & C & SNAPf & absent & pDM104 + SNAPf \\
\hline 116 & PHB2 & C & SNAPf & absent & pDM106 + SNAPf \\
\hline 117 & PHB2 & $\mathrm{N}$ & DK & absent & SDM on pDM112, no gRNA1 site \\
\hline 118 & PHB3 & $\mathrm{N}$ & DK & absent & SDM on pDM112, no gRNA2 site \\
\hline 119 & PHB4 & $\mathrm{N}$ & DK & absent & SDM on pDM112, no gRNA3 site \\
\hline 120 & TFAM & C & SNAPf & present & pDM63 + SNAPf \\
\hline 121 & VIM & C & CLIP & present & pDM101 + CLIP \\
\hline 122 & VIM & C & SNAPf & present & pDM101 + SNAP \\
\hline 123 & ATP5B & C & Dreiklang & absent & Gibson Assembly \\
\hline 124 & ATP5A1 & C & SNAPf & absent & pDM4 + SNAP \\
\hline 125 & ATP5B & C & SNAPf & absent & pDM123 + SNAP \\
\hline
\end{tabular}

Table 15: Overview of all generated monoclonal cell lines. 24 cell lines were generated over the course of this study using CRISPR-Cas9. HET: Heterozygous; HOM: Homozygous; TBD: To be determined.

\begin{tabular}{llllll}
\hline Gene & Transgene & Clones & Genotyped & HET & HOM \\
\hline ACTB & Dreiklang & 12 & - & TBD & TBD \\
\hline ACTB & rsEGFP2 & 11 & - & TBD & TBD \\
\hline ATP5A1 & Dreiklang & 96 & 24 & 23 & 1 \\
\hline ATP5B & Dreiklang & 144 & - & TBD & TBD \\
\hline H2BJ & Dreiklang & 35 & 12 & 11 & 1 \\
\hline H2BJ & rsEGFP2 & 17 & 17 & 17 & - \\
\hline H2BJ & Halo & 31 & - & TBD & TBD \\
\hline HMGA1 & Dreiklang & 23 & - & TBD & TBD \\
\hline HMGA1 & rsEGFP2 & 12 & 12 & 9 & 2 \\
\hline & & & & &
\end{tabular}




\begin{tabular}{llllll} 
MIC60 & Dreiklang & 3 & 3 & 3 & - \\
\hline PHB1 & Dreiklang & 49 & 49 & 49 & - \\
\hline PHB2 & Dreiklang & 74 & 74 & 70 & - \\
\hline RAB7A & Dreiklang & 32 & - & TBD & TBD \\
\hline RTN4 & Dreiklang & 19 & - & TBD & TBD \\
\hline RTN4 & rsEGFP2 & 3 & - & TBD & TBD \\
\hline TFAM & Dreiklang & 7 & 7 & 7 & - \\
\hline TOMM70A & Dreiklang & 8 & 8 & 8 & - \\
\hline VIM & Dreiklang & 15 & 15 & 15 & - \\
\hline VIM & rsEGFP2 & 21 & 21 & 17 & - \\
\hline VIM & Halo & 3 & 3 & 3 & - \\
\hline VIM & SNAP & 10 & 10 & 10 & - \\
\hline ZYX & Dreiklang & 10 & - & TBD & TBD \\
\hline ZYX & rSEGFP2 & 2 & 2 & 1 & 1 \\
\hline ZYX & Halo & 1 & 1 & - & 1 \\
\hline & & & & &
\end{tabular}




\section{List of figures}

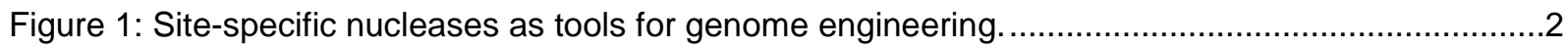

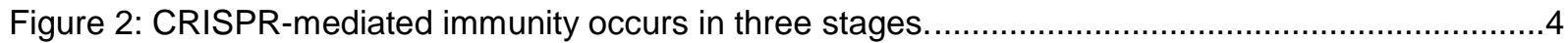

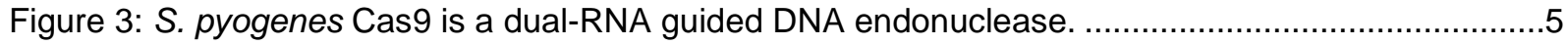

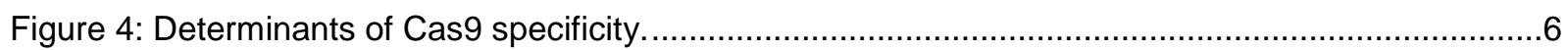

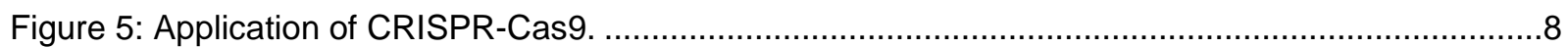

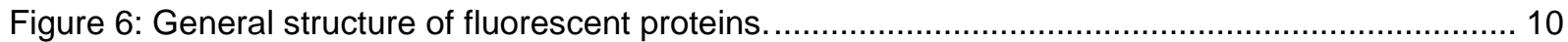

Figure 7: Switching mechanisms in reversibly switchable fluorescent proteins................................ 12

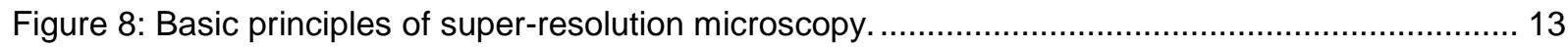

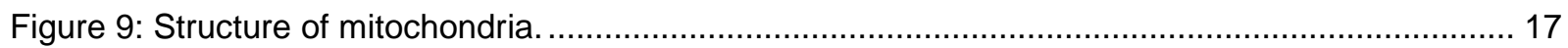

Figure 10: Prohibitins form a mitochondrial membrane complex................................................... 18

Figure 11: PHB complexes as putative membrane scaffolds. .................................................... 20

Figure 12: Zinc finger nucleases targeting the human beta-actin gene. ......................................... 37

Figure 13: Donor plasmids for endogenous tagging of $\beta$-actin.................................................. 38

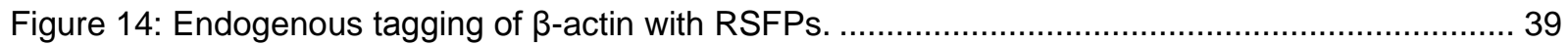

Figure 15: Live-cell RESOLFT nanoscopy on DK-actin cells................................................... 40

Figure 16: Live-cell RESOLFT nanoscopy on rsEGFP2-actin cells.............................................. 41

Figure 17: Schematic for target site selection within a human gene............................................... 42

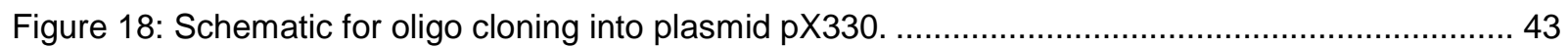

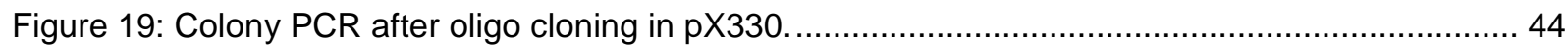

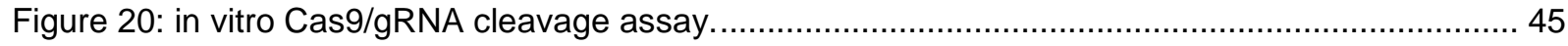

Figure 21: T7 Endonuclease I (T7EI) assay to test Cas9/gRNA activity in vivo. ................................. 46

Figure 22: Two-step four fragment cloning strategy for donor plasmid construction. .......................... 47

Figure 23: One-step Gibson assembly for donor plasmid construction. ............................................. 48

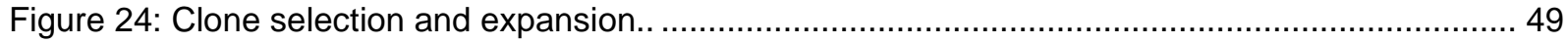

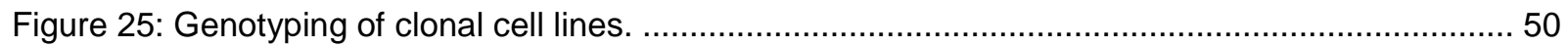

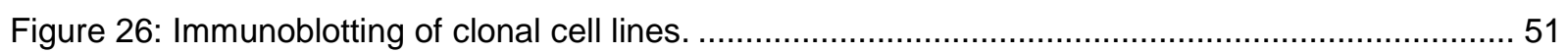

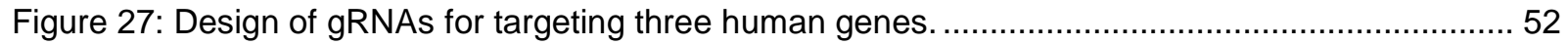

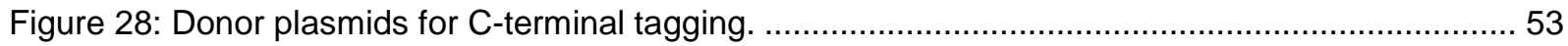

Figure 29: FACS analysis of U2OS cells after nuclease and donor plasmid transfection. .................. 54

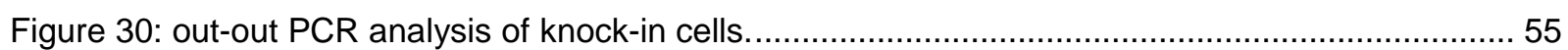

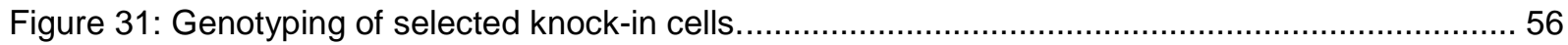

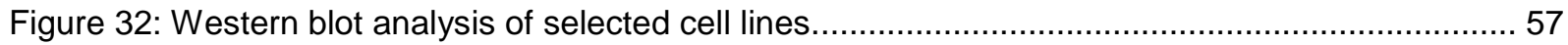

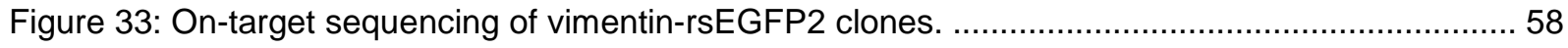

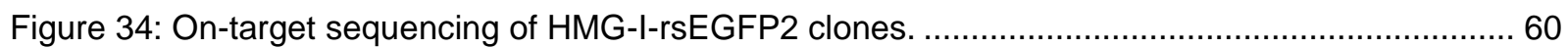

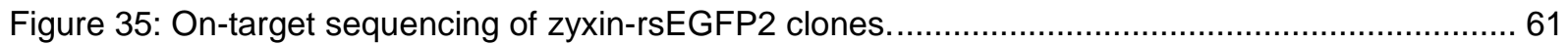




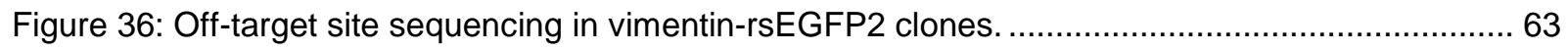

Figure 37: Off-target site sequencing in HMG-I-rSEGFP2 clones................................................. 64

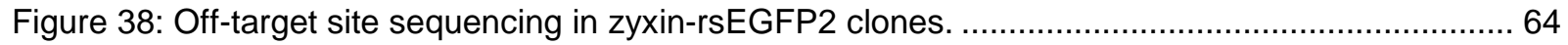

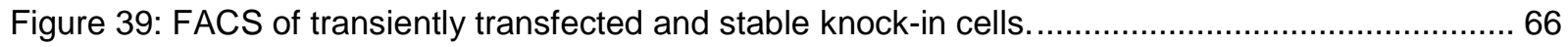

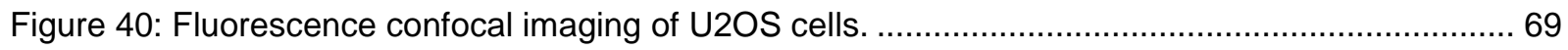

Figure 41: Live cell RESOLFT nanoscopy of vimentin-rsEGFP2 knock-in cells................................. 71

Figure 42: Live cell RESOLFT nanoscopy of HMG-I- and zyxin-rsEGFP2 knock-in cells................... 72

Figure 43: Overexpression of PHB1-DK and PHB2-DK in U2OS cells........................................... 74

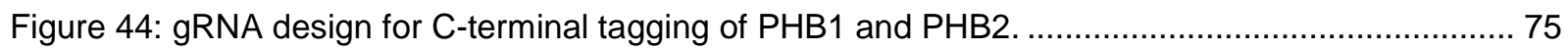

Figure 45: Generation of Cas9-resistant donor plasmids for C-terminal tagging of PHB1 and PHB2... 76

Figure 46: FACS analysis of U2OS cells after co-transfection with PHB1 and PHB2 targeting nuclease

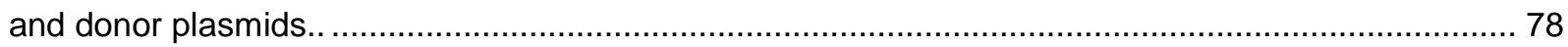

Figure 47: Genotyping of PHB1-DK and PHB2-DK knock-in cells.............................................. 79

Figure 48: Protein expression level analysis in PHB1-DK and PHB2-DK clones. ............................... 80

Figure 49: Confocal microscopy of PHB1-DK and PHB2-DK knock-in cells..................................... 81

Figure 50: On-target sequencing of PHB1-DK and PHB2-DK cell lines. ........................................... 82

Figure 51: DNA sequencing of off-target sites in PHB1-DK and PHB2-DK cells............................... 84

Figure 52: STED microscopy reveals PHB1-DK and PHB2-DK clusters in knock-in cells. .................. 86

Figure 53: STED microscopy reveals PHB1 and PHB2 clusters in wildtype cells. ........................... 87

Figure 54: Co-localization of PHB1-DK with PHB1 and PHB2-DK with PHB2 ................................. 88

Figure 55: Co-localization of PHB1-DK with PHB2 and PHB2-DK with PHB1. ................................ 89

Figure 56: Submitochondrial localization of PHB1-DK and PHB2-DK using quanitative immunoelectron microscopy.

Figure 57: Submitochondrial localization of PHB1 and PHB2 in wildtype cells using quanitative

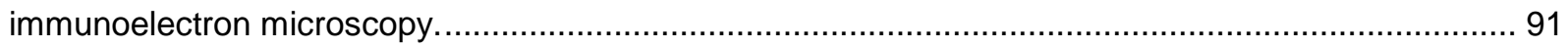

Figure 58: PHB1-DK and PHB2-DK are localized in immobile fractions........................................... 93

Figure 59: CRISPR-mediated endogenous PHB2-tagging in HeLa cells. .......................................... 94

Figure 60: Genotyping of HeLa-PHB2-Dreiklang clones using PHB2 locus-specific out-out PCR....... 95

Figure 61: PHB2 overexpression induces nuclear mislocalization in HeLa cells.............................. 96

Figure 62: Estimation of PHB1 and PHB2 amounts per cell using recombinant DK. ........................ 98

Figure 63: Estimation of PHB1 and PHB2 amounts per cell using recombinant PHB1 or PHB2 for

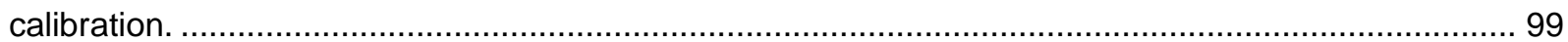

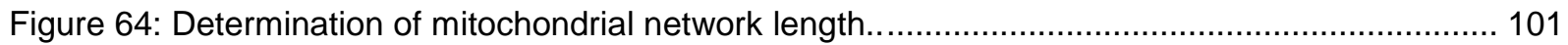

Figure 65: Determination of inter-cristae distance. ................................................................... 102 


\section{List of tables}

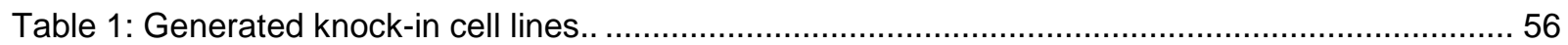

Table 2: Overview of the top two predicted off-target sites associated with each gRNA $\ldots \ldots \ldots \ldots \ldots \ldots \ldots \ldots . \ldots 2$

Table 3: Predicted off-target sites for PHB1-gRNA2 and PHB2-gRNA2 ........................................ 83

Table 4: Primers used for one-step cloning of overexpression plasmid. ........................................... 143

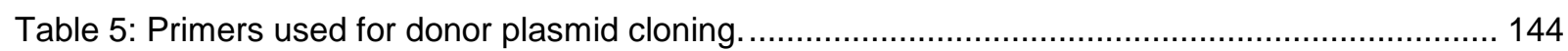

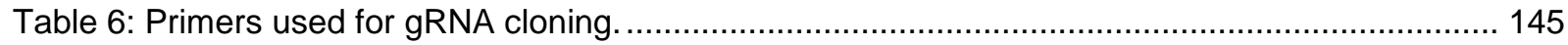

Table 7: Plasmid templates and primers used to generate T7-gRNA templates for IVT of gRNAs. ... 146

Table 8: Primers used for amplification of DNA substrates to test gRNAs in vitro. .......................... 146

Table 9: Primers used for amplification of DNA substrates for T7 Endonuclease I assay.................. 146

Table 10: Primers used for on-target site analysis via PCR and DNA sequencing. ........................ 146

Table 11: Primers used for off-target site analysis via DNA sequencing ...................................... 147

Table 12: Overview of parameters used for RESOLFT imaging of endogenously tagged cells......... 147

Table 13: Overview of all generated gRNA/Cas9 plasmids (pMR) during this study........................ 148

Table 14: Overview of all generated donor plasmids (pDM) within this study. ................................ 154

Table 15: Overview of all generated monoclonal cell lines......................................................... 156 


\section{Abbreviations}

AOTF

APD

APS

ATP

bp

BSA

Cas

cDNA

CJ

CLSM

CM

CRISPR

$\mathrm{Da}$

DABCO

DAPI

DK

DMEM

DNA

dNTP

E. coli

EDTA

EGFP

ER

FA

FACS

FBS

FP

FRAP

FWHM

GA

GFP
Acousto-optical tunable filter

Avalanche Photodiode

Ammonium persulfate

Adenosine triphosphate

Base pair

Bovine serum albumin

CRISPR-associated

Coding deoxyribonucleic acid

Cristae junction

Confocal Laser Scanning Microscopy

Cristae membrane

Clustered regularly interspaced short palindromic repeats

Dalton

1,4-diazabicyclo[2.2.2]octan

4',6-diamidino-2-phenylindole

Dreiklang

Dulbecco's Modified Eagle's Medium

Deoxyribonucleic acid

Deoxynucleoside triphosphate

Escherichia coli

Ethylenediaminetetraacetic acid

Enhanced green fluorescent proteins

Endoplasmic reticulum

Formaldehyde

Fluorescence activated cell sorting

Fetal bovine serum

Fluorescent protein

Fluorescence recovery after photobleaching

Full width half maximum

Glutaraldehyde

Green fluorescent protein 


\begin{tabular}{|c|c|}
\hline gRNA & Guide RNA \\
\hline HET & Heterozygous \\
\hline HMGA1 & High mobility group AT-hook 1 \\
\hline HOM & Homozygous \\
\hline IMM & Inner mitochondrial membrane \\
\hline IPTG & Isopropyl- $\beta$-D-thiogalactopyranosid \\
\hline IVT & in vitro transcription \\
\hline kDa & Kilodalton \\
\hline KFP1 & Kindling fluorescent protein \\
\hline LB & Luria Bertani \\
\hline $\min$ & Minute \\
\hline MUT & Mutant \\
\hline NA & Numerical aperture \\
\hline OD & Optical density \\
\hline OMM & Outer mitochondrial membrane \\
\hline OPA1 & Optic atrophy 1 \\
\hline PAGE & Polyacrylamide gel electrophoresis \\
\hline PALM & Photoactivation localization microscopy \\
\hline PBS & Phosphate-buffered saline \\
\hline PCR & Polymerase chain Reactionr \\
\hline PFA & Paraformaldehyde \\
\hline PHB & Prohibitin \\
\hline PMT & Photomultiplier tube \\
\hline PSF & Point Spread Function \\
\hline RESOLFT & Reversible Saturable Optical Fluorescence Transition \\
\hline RNA & Ribonucleic acid \\
\hline ROS & Reactive oxygen species \\
\hline rsEGFP & Reversibly switchable enhanced green fluorescent protein \\
\hline RSFP & Reversibly switchable fluorescent protein \\
\hline SAM & Sorting and assembly machinery \\
\hline S. pyogenes & Streptococcus pyogenes \\
\hline SDS & Sodium dodecyl sulfate \\
\hline sec & Second \\
\hline STED & Stimulated emission depletion \\
\hline
\end{tabular}




$\begin{array}{ll}\text { STORM } & \text { Stochastic optical reconstruction microscopy } \\ \text { TBS } & \text { Tris-buffered saline } \\ \text { TBST } & \text { Tris-buffered saline and Tween } 20 \\ \text { TEM } & \text { Transmission electron microscopy } \\ \text { TEMED } & \text { Tetramethylethylenediamine } \\ \text { TIM } & \text { Translocase of the inner membrane } \\ \text { TOM } & \text { Translocase of the outer membrane } \\ \text { Tris } & \text { Tris(hydroxymethyl)aminomethane } \\ \text { UAC } & \text { Uranyl acetate } \\ \text { UV } & \text { Ultraviolet } \\ \text { VIM } & \text { Vimentin } \\ \text { V/V } & \text { Volume/volume } \\ \text { WB } & \text { Western blot } \\ \text { WT } & \text { Wildtype } \\ \text { W/V } & \text { Weight/volume } \\ \text { YFP } & \text { Yellow Fluorescent Protein } \\ \text { ZYX } & \text { Zyxin } \\ & \end{array}$




\section{Acknowledgements}

First and foremost, I would like to thank my supervisor, Prof. Stefan Jakobs, for giving me the great opportunity to join his lab and to independently work on the presented topics. His insightful advice and constant support were crucial for making this work possible.

I am thankful to Prof. Peter Rehling who was a valuable asset as a thesis committee member and an inspiring discussion partner. His feedback and support were critical to shape the course of my scientific projects.

I am grateful to Prof. Stefan W. Hell for the unique opportunity to join his department during exciting times and his fundamentally inspiring way to see the wood for the trees.

Moreover, I would like to thank Prof. Michael Meinecke, Prolf. Detlef Doenecke and Prof. Markus T. Bohnsack for being part of my extended thesis committee.

I would like to give special thanks to Prof. Ilaria Testa, the queen of RESOLFT nanoscopy. I admire her art of making things look easy, that others would not even dare to try.

Many thanks go to Dr. Dietmar Riedel and Dr. Dirk Wenzel for contributing essential electron microscopy images to this work and helpful discussions on that topic.

I am thankful to Sabrina Becker from the University Medical Center, who was the irreplaceable flow cytometrist that mastered the skill of single human cell sorting.

I want to thank Tanja Gilat, Ellen Rothermel, Sylvia Löbermann, Rita Schmitz-Salue and Maria Sermond for excellent technical assistance and keeping everything perfectly tidy in the lab. A big thank you goes to Jaydev Jethwa for help on proofreading my first paper, soldering guitar wiring, fixing glasses... and for sharing exciting stories about MPI-BPC since 1971.

Thanks to Kerstin Grüninger and Steffen Burkhardt from the IMPRS MolBio office for bringing me together with students from all over the world and for their exceptional motivation to organize conferences, retreats, exchange trips and many more events.

I would like to thank all former and present members of the Department for NanoBiophotonics for a relaxed working atmosphere. Especially, I am thankful to the Dynamitos soccer team for biannual training efforts that were sufficient to win the MPI cup two times in a row.

Special thanks go to trusted friends in Göttingen for constant support, good fun and sharing a passion for music, soccer, bike trips, and decadent Italian food.

I am forever indebted to Ilaria for being the brilliant partner at my side that supported me no matter how good, bad or ugly things were.

Finally, I want thank my entire family for all their love and support, patience and encouragement beyond any description. 


\section{Affidavit}

Herewith I declare, that I prepared the PhD thesis entitled

"CRISPR-Cas9-mediated protein tagging in human cells for RESOLFT nanoscopy and the analysis of mitochondrial prohibitins"

on my own and with no other sources and aids than quoted.

Göttingen, $12^{\text {th }}$ November 2015

Michael Ratz 


\section{Curriculum vitae - Michael Ratz}

born on $17^{\text {th }}$ January 1986 in Sangerhausen, Germany

University education

Max-Planck-Institute for Biophysical Chemistry, Germany

$07 / 2012-12 / 2015$

$\mathrm{PhD}$, Molecular Biology

University of Göttingen, Germany

$10 / 2010-03 / 2011$

MSc, Molecular Biology

University of Leipzig, Germany

$10 / 2007-09 / 2010$

BSc, Biochemistry

Research experience

Max-Planck-Institute for Biophysical Chemistry, Germany

$07 / 2012-12 / 2015$

- PhD research in the Department of NanoBiophotonics under supervision of Prof. Stefan Jakobs: "CRISPR-Cas9-mediated protein tagging for RESOLFT nanoscopy and the analysis of mitochondrial prohibitins"

Max-Planck-Institute for Biophysical Chemistry, Germany

$04 / 2012-06 / 2012$

- Internship in the Department of NanoBiophotonics (Prof. Stefan Jakobs \& Prof. Stefan W. Hell)

Max-Planck-Institute for Biophysical Chemistry, Germany

$10 / 2011-03 / 2012$

- Master thesis in the lab of Prof. Dirk Görlich: Characterization of nucleoporin-specific viral proteases.

Göttingen Center for Molecular Biosciences (GZMB)

$05 / 2011-06 / 2011$

- Lab rotation project in the lab of Prof. Ralf Ficner: Investigation of interactions among S. cerevisiae elF3 subunits Nip1, Tif32 and Prt1.

Max-Planck-Institute for Biophysical Chemistry, Germany

$03 / 2011-04 / 2011$

- Lab rotation project in the lab of Prof. Holger Stark: Resolving the structure of the E. coli pyruvated dehydrogenase complex E2 core by single particle negative stain electron microscopy.

Max-Planck-Institute for Biophysical Chemistry, Germany

$01 / 2011-02 / 2011$

- Lab rotation project in the lab of Prof. Dirk Görlich: Cloning of Nup153, and expression and purification of Nup93 chimeras fused to various GLFG repeats.

University of Leipzig, Germany

$03 / 2010-06 / 2010$

- Bachelor thesis in the lab of Prof. Mario Mörl: Creation and characterization of a TPP riboswitch deletion variant. 


\section{Publications}

Ratz, M., Testa, I., Hell, S.W., and Jakobs, S. (2015): CRISPR/Cas9-mediated endogenous protein tagging for RESOLFT super-resolution microscopy of living human cells. Scientific Reports 5, 9592.

Chmyrov, A., Keller, J., Grotjohann, T., Ratz, M., d'Este, E., Jakobs, S., Eggeling, C., and Hell, S.W. (2013): Nanoscopy with more than 100,000 'doughnuts'. Nature Methods 10, 737-740.

\section{Awards and distinctions}

- PhD Fellowship (Max Planck Society, Germany)

$07 / 2012$ - 12/2015

- Best Student Talk (FEBS Young Scientists' Forum, Berlin,

$07 / 2015$ Germany)

- Travel Grant (FEBS Young Scientists' Forum, Berlin, Germany)

$07 / 2015$

- Travel Grant (Assembly and Disassembly of the Nervous System, Weizmann Institute of Science, Rehovot, Israel)

- $\quad$ Stipend of the Excellence Foundation for the promotion of the Max Planck Society (International Max Planck Research School (IMPRS) Molecular Biology, University of Göttingen, Germany)

\section{Skills and techniques}

- Genome-editing: CRISPR-Cas9, TALENs and ZFNs in mammalian cells

- Fluorescence microscopy: (Live cell) Confocal, RESOLFT, STED, PALM

- Molecular biology: PCR (real-time, quantitative, digital), Cloning, DNA/RNA isolation and analysis, DNA sequencing, in vitro transcription and translation

- Cell biology: Mammalian cell culture, transfection, Western blot, IP

- Protein biochemistry: Protein expression and purification, Chromatography, FRET-based assays for protease activity monitoring in vitro

- Programming skills: R, Python, MATLAB

- Language skills: German (native speaker), English (fluent)

\section{Methods courses}

\section{GGNB Graduate School Short Methods Courses (2-4 days each)}

- Optics in Microscale to Nanoscale: From Rainbow to Plasmonic

$06 / 2014$

- Next Generation Sequencing (NGS) - Analysis of RNAseq and

$04 / 2014$ ChIPseq data 
- Protein Family Analysis

$04 / 2014$

- Theory and basics of fluorescence microscopy imaging

09/2013

- Reconsitution of neuronal exocytosis

$11 / 2012$

- Genotyping using FRET on the LightCycler

$03 / 2012$

- Nerve cell culture and patch-clamp recording from nerve cells

$11 / 2012$

Certified online courses

- HarvardX: PH525.2x: Matrix Algebra and Linear Models

16.02. - 01.03.14

- HarvardX: PH525.1x: Statistics and R for the Life Sciences

19.01. - 15.02.15

- MITx 7.QBWx: Quantitative Biology Workshop

10.06. - 30.07.14

\section{Conferences and workshops}

$40^{\text {th }}$ FEBS Congress and $15^{\text {th }}$ Young Scientists' Forum. Berlin, Germany

02. -09.07 .15$

- Short talk (YSF) and poster (FEBS): CRISPR/Cas9-mediated endogenous protein tagging for super-resolution microscopy and its application for studying mitochondrial dynamics

Assembly and Disassembly of the Nervous System.

19. - 21.01.15

Weizmann Institute of Science, Rehovot, Israel

- Short talk: CRISPR/Cas9-mediated endogenous protein tagging for RESOLFT super-resolution microscopy of living human cells

From Functional Genomics to Systems Biology.

08. - 11.11.14

EMBL Heidelberg, Germany.

Labeling and Nanoscopy. DKFZ Heidelberg, Germany

24. - 26.09.14

GGNB Science Day 2013. MPIbpc Göttingen, Germany

09.12 .13

- Poster: Endogenous gene tagging with fluorescent reporters using the CRISPR-Cas9 nuclease system

Precision Genome Engineering and Synthetic Biology: Designing

17. - 22.03.13

Genomes and Pathway. Breckenridge, CO, USA

- Poster: Endogenous tagging of mitochondrial proteins for fluorescence nanoscopy

EMBO Workshop. EMBL Heidelberg, Germany

26. - 28.02.13

- Development of Transgenic Animals Using Zinc Finger Nucleases

Teaching and supervision

University of Göttingen, Germany

$05 / 2014$

Course advisor, Albrecht-von-Haller-Institut for Plant Sciences

- Advised medical students on basic molecular biology methods 


\section{Max-Planck-Institute for Biophysical Chemistry, Germany}

Supervisor of two lab rotation students, Department of

NanoBiophotonics

$05 / 2014-06 / 2014$

- Indira Memet: Endogenous gene-tagging with fluorescent reporters

in mammalian cells using the CRISPR/Cas9 nuclease system.

$03 / 2015-04 / 2015$

- Mohamed El-Brolosy: Overexpression analysis and CRISPR/Cas9mediated endogenous protein tagging of prohibitins in human cells.

University of Göttingen, Germany

$03 / 2011-11 / 2014$

Teaching Assistant, IMPRS Molecular Biology

- Taught advanced classes in molecular biology

\section{Conference organization}

Horizons in Molecular Biology 2012 \& 2013

$10 / 2011-09 / 2013$

- Conference planning and organization with fellow PhD students

- Initiated, planned and participated in production of promotional trailer: http://vimeo.com/53509540 